
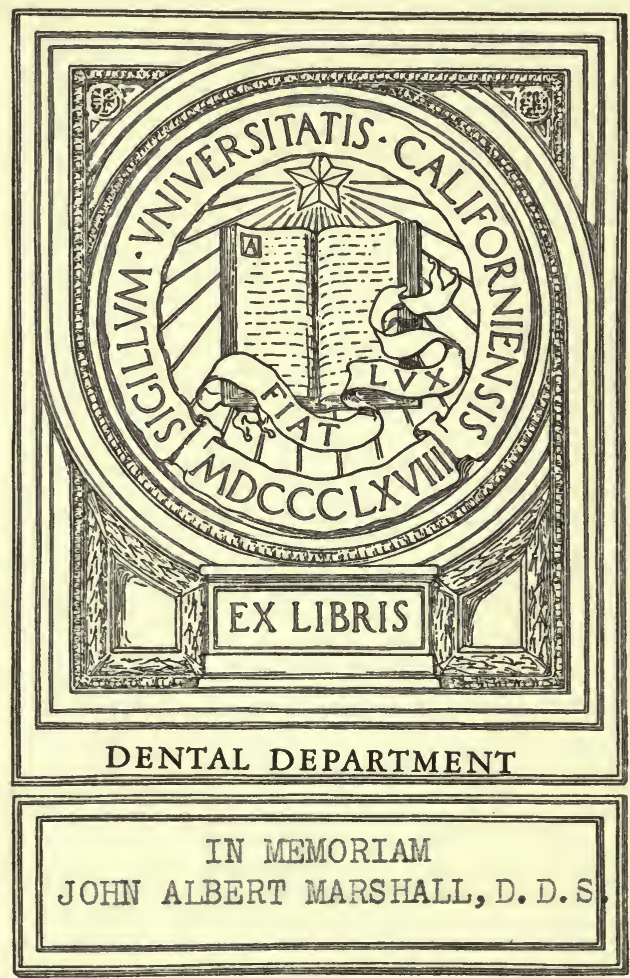
Digitized by the Internet Archive in 2007 with funding from Microsoft Corporation 



\section{PHYSIOLOGICAL CHEMISTRY}

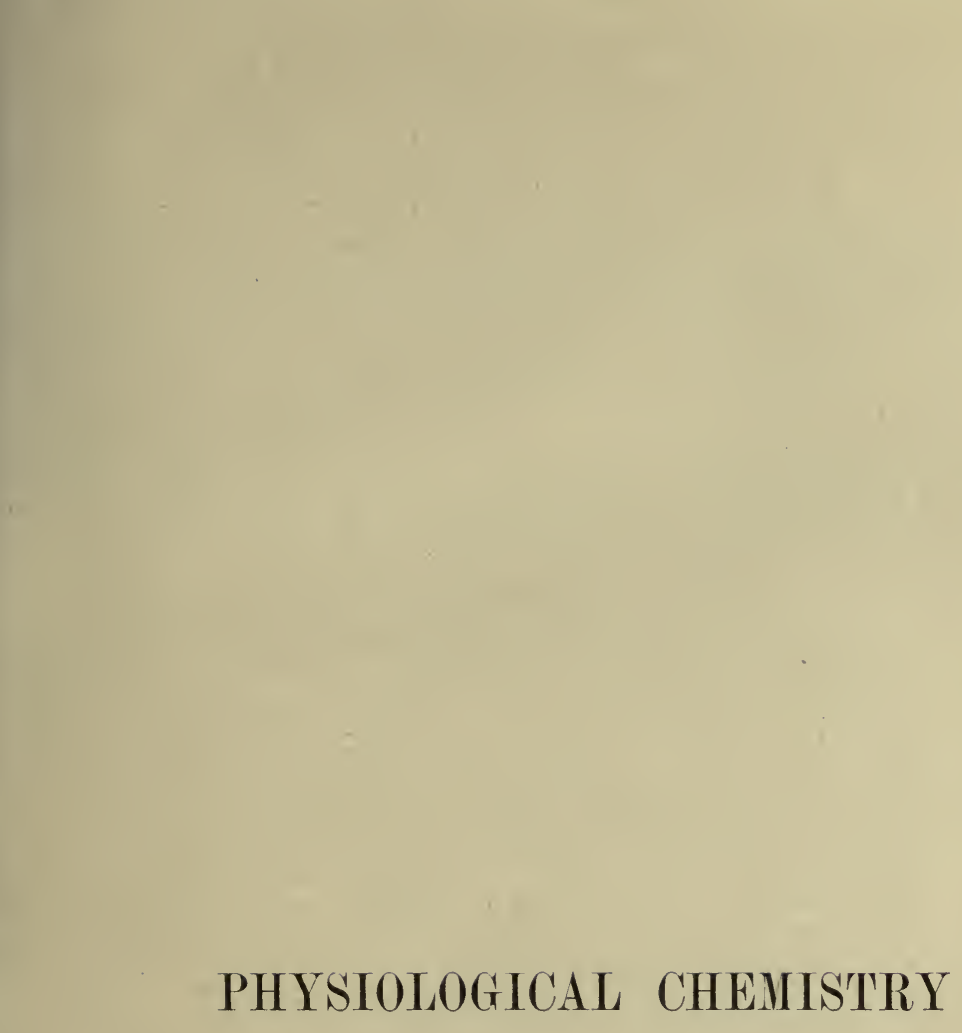





\section{AN INTERMEDIATE TEXTBOOK}

\section{OF \\ PHYSIOLOGICAL CHEMISTRY}

\section{WITH EXPERIMENTS}

BY

C. J. V. PETTIBONE, Рн.D.

ASSOCIATE PROFESSOR OF PIYYSIOLOGICAL CHEMISTRY, MEDICAL SCHOOL, UNIVERSITY OF MINNESOTA, MINNEAPOLIS

SECOND EDITION

ST. LOUIS

C. V. MOSBY COMPANY

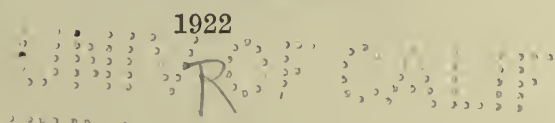


Copyright, 1917, 1922, By C. V. Mosby Company

(Printed in U. S. A.) 
M. A. P., I. P. H., AND M. P. G.

TO
M. A. P., I. P. H., ANd M. P. G.

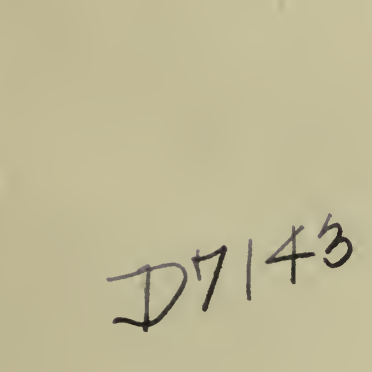

\section{$Q P 51^{4}$
$P 52$
1922 \\ $Q P 51^{4}$
$P 52$
1922 \\ $Q P 51^{4}$
$P 52$
1922 .}

-

$-$

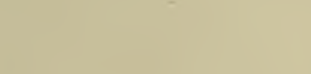

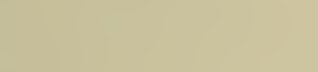

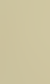





\section{PREFACE TO SECOND EDITION}

In preparing the second edition of this book the author has attempted to preserve the original character of the work. Much material has been added, however, both in the text and laboratory section, and some older methods have been omitted. A chapter on the portions of physical chemistry of most interest in biology has been added, including more extended discussion. of colloids, and the material on enzymes has been considerably extended. More material on the saliva, and muscle has been included and a summary of the vitamines to the present date. In the laboratory section the principal additions are the segregation of the physicochemical material with the addition of various simple but significant experiments, and the inclusion of Folin's methods for blood analysis and microchemical estimation of certain constituents of the urine.

The author wishes to express thanks for various suggestions and corrections.

Minneapolis, August, 1922.

$$
\text { C. J. V. P. }
$$

\section{PREFACE TO FIRST EDITION}

My aim in writing this book has been to prepare an intermediate text which would cover the general field of physiological chemistry in such a way as to give students a familiarity with compounds important from a biochemical viewpoint, and to acquaint them with the fundamental processes which go on in the animal body. I have attempted to avoid confusing the beginner with lengthy discussions of debated points, but to set forth as clearly as possible the present status of our knowledge. The material is so chosen that the book may be used for intermediate classes, or for advanced work if supplemented by lectures. 
The appended laboratory work has been drawn from the manual in use in my classes for the last five years. Much of the material has, of course, been drawn from other manuals.

I wish to acknowledge suggestions and corrections in the laboratory directions from various colleagues, particularly Dr. F. B. Kingsbury. I wish also to express my thanks to Dr. W. H. Hunter, who kindly volunteered to read the manuscript of the theoretical portion.

Minneapolis, Minn.

C. J. V. Pettibone. 


\section{CONTENTS}

\section{PART I}

\section{INTRODUCTORY}

Object and Importance of Physiological Chemistry, 17 ; Protoplasm, 18; Material Bases, 19.

\section{CHAPTER I}

Physical Chemistry in Its Relations to Physiological Chemistry

Importance, 21; Osmotic Pressure, 21; Electrical Properties of Solutions, 23; H-ion Concentration, 23; Titratable Acidity, 25; Colloidal Solutions, 28; Classification and Properties of Colloids, 29; Tyndall's Phenomenon, 30 ; Electrical Properties of Colloids, 31; Methods of Precipitating Colloids, 32; Absorption-Surface Tension, 33; Imbibition, 34.

\section{CHAPTER II}

Elements, Inorganic Matfrials, Water

Elements Found in the Body, 35; Importance not Determined by Amount Present, 35; Carbon, Hydrogen, Oxygen, Nitrogen, Sulphur and Phosphorus, 36; Sodium, Potassium, Calcium, Magnesium and Iron, 38; Chlorine, Iodine, Fluorine, ete., 40; Water, 40.

\section{CHAPTER III}

\section{Carbohydrates}

Composition, Oceurrence, General Function, 41; Structure of the Carbohydrates, 41; Optical Activity, 42; Classification of Carbohydrates, 48; Origin and Synthesis, 49; Interconversion of Carbohydrates, 51; Combination of Carbohydrates with One Another, and with Other Substances, 52; Behavior with Strong Alkalies, 52; Behavior with Acids, 53; Oxidation of Carbohydrates, 54; Reduction of Carbohydrates, 57; Formation of Osazones, 57; The Molisch Test, 58; Fermentation-Enzymes, 59; Nomenelature and Classification, 62; Specific Nature, 63; Influence of Temperature, 64; Effect of Chemical Reaction, 64; Reversibility, 65; Active and Inactive 
Form, 65; Action Retarded by Products, 66; Progressive Action, 66; Antienzymes-Defensive Enzymes, 66; Summary, 66; Individual Groups of Carbohydrates, 67 ; Pentoses, 67 ; Absorption Spectra, 68; Hexoses. $\mathrm{C}_{6} \mathrm{H}_{12} \mathrm{O}_{6}$, 69 ; Glucose, 69 ; Fructose. (Levulose, Fruit Sugar), 70; d-Galactose, 70; Amino Sugars, 71; d-Glycuronic Acid, 71; Disaccharides, 72; Saccharose. (Sucrose, Cane Sugar), 72 ; Lactose, 74 ; Maltose, 75 ; Polysaccharides, 75 ; Starch $\left(\mathrm{C}_{6} \mathrm{H}_{10} \mathrm{O}_{5}\right)_{n}, 76$; Dextrins, 77 ; Inulin, 77; Gums and Mucilages, 77 ; Cellulose, 77 ; Glycogen, 78 ; Glucosides, 79.

\section{CHAPTER IV}

\section{Fats, Phosphatids, and Allied Substances}

Distribution and Importance, 80; Composition and Structure, 80; General Properties, 82 ; Emulsification, 82 ; Saponification, 83 ; Rancid Fats, 84; Detection and Identification, 84; Important Fats, 86; Phosphatids, Cerebrosides, and Sterols, 87; Lecithin, 88; Cholesterol, 89.

\section{CHAPTER V}

\section{Proteins}

Introductory, 91; Elementary Composition, 91; Classification, 92; Preparation of Proteins from Materials in Which They Oceur, 93; Molecular Weight, 94; Hydrolysis, 94; Amino Acids Obtained by Hydrolyzing Protein, 95; General Properties and Reactions of Amino Acids, 98; General Protein Reactions, 101; Color Tests, 101; Precipitation Reactions, 103; Structure of the Protein Molecule, 105; Putrefaction of Proteins, 109; Individual Groups, Simple Proteins, 110; Albumins, 111; Globulins, 111; Glutelins, 112 ; Prolamines, 112; Albuminoids, 112; Histones, 113 ; Protamines, 113; Conjagated Proteins, 113; Glycoproteins, 114; Phosphoproteins, 114 ; Hemoglobins, 116; Detection of Hemoglobin, 118; Absorption Spectra of Oxyhemoglobin and Hemoglobin, 120; Derivatives of the Hemoglobins, 121; Fate of Blood Pigment in the Body, 123; Nucleoproteins, 123; Lecithoproteins, 125; Derived Proteins, 125; Primary Protein Derivatives, 125 ; Secondary Protein Derivatives, 127.

\section{CHAPTER VI}

\section{Some FAMiliar FoodstufFs-SOME Important Tissues}

Some Important Foodstuffs, 130; Cooking and Preparation of Foods, 131; Milk, 131; Butter, 132; Cheese, 133; Meats, 133; Eggs, 133; Vegetables, 134; Breadstuffs, 134; Choice of Diet, 135; Some Important Tissues, 135; Muscle, 135; Brain and Nerves, 138; Bones and Teeth, 138; Connective Tissue, 139; The Blood, 140; Reaction of the Blood, 142; Os- 
motic Pressure, 143; Coagulation of the Blood, 143; Lymph, 144; The Skin, 144.

\section{CHAPTER VII}

Digestion IN THE MOUTH

General Purpose of Digestion, 145; Preparation of Food, 146; Saliva, 146.

\section{CHAPTER VIII}

\section{Digestion IN THE StomaCH}

Importance, 152; Methods of Study, 152; Causation of Flow of Gastric Juice, 153; General Character of the Secretion, 155; Hydrochloric Acid, 155; Source of the Hydrochloric Acid, 157; The Functions of the Hydrochloric Acid, 157; Enzymes of the Gastric Juice, 158; Pepsin, 158; Products of Peptic Digestion, 159; Rennin, 159; Are Pepsin and Rennin Identical? 160; The Stomach Wall is not Digested, 161; Passage of the Food into the Intestine, 161.

\section{CHAPTER IX}

\section{Digestion IN THE INTESTINE}

General, 162; Pancreatic Juice, 162; General, 162; Mechanism of Flow, 163; Composition of Pancreatic Juice, 163; Trypsin, 164; Rennin, 165 ; Action on Fats, 165 ; Steapsin, 165 ; Action on Starches, 165; Amylase or "Amylopsin," 165; The Bile, 166; Causes of Flow, 166; Amount, 166; Composition. Function, 167; Bile Pigments, 167; Bile Salts, 168; Intestinal Secretion, 168; Erepsin, 169; Other Enzymes, 169; Excretory Funetion of Intestinal Secretion, 169; Bacterial Action in the Intestine, 170; Feces, 171.

\section{CHAPTER $\mathrm{X}$}

\section{ABSORPTION}

General, 173; Absorption of Proteins, 174; Carbohydrate Absorption, 174; Absorption of Fats, 175.

\section{CHAPTER XI}

\section{URINE}

General, 176; Physical Properties, 177; Volume, 177; Color, Transparency, 178; Consistency, Odor, Taste, 179; Specific Gravity.-Total Solids, 179; Optical Activity, Reducing Power, Fermentation, ete., 180; Reaction, 181; Urea, 182; Uric Acid and Other Purine Derivatives, 184; Hippuric Acid, 187; Ammonia, 188; Creatinine and Creatine, 188; Inorganic Constituents, 190; Chlorides, 190; Phosphates, 191; Sulphates, 191; 
Carbonates, 193; Sodium, Potassium, Calcium and Magnesium, 193; Pathological Constituents of the Urine, 193.

\section{CHAPTER XII}

\section{Metabolism}

General, 194; Protein Metabolism, 195; Amount of Protein Required. Nitrogen Balance, 197; Carbohydrate Metabolism, 203; Sources of Glycogen, 209; Metabolism of Fats, 211; Metabolism of Inorganic Material, 213; Energy Exchange, 214; Utilization of Alcohol by the Body, 220; Starvation, 220; Unknown Food Constituents.-Vitamins, 221; Body Temperature, 225; Influence of Organs of Internal Secretion, 225.

\section{PART II}

\section{LABORATORY WORK}

Materials, 228; General Laboratory Instructions, 234.

\section{CHAPTER I}

\section{STANDARD ACID AND AlKali}

Physical Chemistry-Calibration of Volumetric Apparatus, 236; Normal Acid and Alkali, 236; Oxalic Acid Method, 237; Sodium Carbonate Method, 238; Preparation of N/10 Acid, 239; Preparation of a Standard Alkali, 240; Kjeldahl Method for Nitrogen Determination, 241; Indicators, 244; Catalysis, 244; Colloids, 245 ; Solation and Gelation, 245; Imbibition, 245; Dialysis, 245; Suspensoids and Emulsoids, 245; Reversibility, 245; Diffusion, 245; Absorption, 246.

\section{CHAPTER II}

\section{Detection of the Elements and of Inorganic Salts}

Carbon, 247; Hydrogen, 247; Oxygen, 247; Nitrogen, 248; Sulphur, 248; Preparation of Muscle Extract, 248; Muscle Residue, 249; Blood Serum, 249; Bone, 250; Tests for Inorganic Materials, 250; Chlorides, 250; Sulphates, 251; Phosphates, 251; Carbonates, 251; Calcium, 251; Magnesium, 251; Iron, 252; Sodium, 252; Potassium, 253.

\section{CHAPTER III}

\section{CARbohydRates}

Monosaccharides, 254; Dextrose, 254; Benedict's Qualitative Reagent for Sugar, 255; Levulose, 258; Galactose, 259; Arabinose, 259; Disac- 
charides, 260; Saccharose, (Sucrose), 260; Maltose, 260; Lactose, 261; Polysaccharides, 261; Starches, 261; Dextrines, 262; Glycogen, 263.

\section{CHAPTER IV}

Fats and Pihosphatides

Fats, 264; Phosphatides, 267; Lecithin, 267.

\section{CHAPTER V}

\section{Proteins}

General Protein Reactions, 269; Color Reactions, 269; Precipitation Tests, 271; Individual Groups-Simple Proteins, 274; Albumins, 274; Globulins, 275; Prolamines, 276; Gluteins, 277; Albuminoids, 277; Histones, 278; Protamines, 278; Conjugated Proteins, 279; Glycoproteins, 279 ; Hemoglobins, 279; Phosphoproteins, 286; Nucleoproteins, 288; Derived Proteins, 289; Primary Protein Derivatives, 289; Proteans, 289; Metaproteins, 289; Coagulated Proteins, 291; Secondary Protein Derivatives, 291; Proteoses, 291; Peptones, 292; Peptids, 293; Amino Acids, 293.

\section{CHAPTER VI}

\section{Microchemical Methods for Blood Analysis}

Folin's Modified Nessler's Reagent, 296; Preparation of Protein-Free Blood Filtrates, 297; Nonprotein Nitrogen Determination, 299; Determination of Urea of Urease Decomposition and Distillation, 300; Determination of Preformed Creatinine, 302; Determination of Creatine Plus Creatinine, 303; Determination of Uric Acid in Blood, 304; Blood Sugar, 306; Method for the Determination of Chlorides in Blood Plasma, 309.

\section{CHAPTER VII}

\section{SALIVARY Digestion}

Composition, 310; Digestive Action, 311.

\section{CHAPTER VIII}

\section{Gastric Digestion}

Preparation of Artificial Gastrie Juice, 314; Composition of Gastric Juice, 314; Digestive Action of Gastric Juice, 316; Motor Power of the Stomach, 318; Rate of Absorption from the Stomach, 318. 


\section{CHAPTER IX \\ Pancreatio Digestion-Bile}

Pancreatic Juice, 319; Composition of Pancreatic Juice, 319; Digestive Action, 319; Intestinal Juice, 320; Bile, 320; Composition, 320; Effect of Bile on Surface Tension, 321; Biliary Calculi, or Gall Stones, 321.

\section{CHAPTER $\mathrm{X}$}

\section{URINE}

Qualitative Study, 323; Inorganic Constituents, 323; Organic Constituents, 325; Collection and Preservation of a Specimen for Quantitative Analysis-General Properties, 328; Quantitative Analysis (Make All Determinations in Duplicate), 331; Total Solids, 331; Total Nitrogen, 332; Ammonia, 332; Urea, 334; Colorimetric Method for Determination of Urea in Urine, 335; Uric Acid, 337; Hippuric Acid, 340; Purine Bases, 340; Creatinine (Folin), 340; Microchemical Determination of Creatinine in Urine, 342; Indican, 343; Allantoin, 343; Oxalic Acid, 343; Chlorides, 343; Sulphates, 344; Phosphates, 345; Pathologic Urine, 346; Carbohydrates, 349 ; Acetone Bodies, 353.

\section{APPENDIX}

\section{Directions for Making Up Quantitative on Speciat، Reagents}

Ammonium Thiocyanate, Standard, for Chlorides, 354; Barfoed's Solution, 354; Barium Chloride for Sulphate Determination, 354; Benedict's Solution for Carbohydrate Estimation, 355; Congo Paper, 355; Esbach's Reagent, 355; Fehling's Solution (Quantitative), 355; Fehling's Solution (Qualitative), 355; Folin-Schaffer Reagent, 355; Formalin (Neutral), 356; Glyoxylic Acid Solution, 356; Guenzberg's Reagent, 356; Magnesia Mixture, 356; Mett's Tubes, 356; Millon's Reagent, 356; Molisch's Reagent, 357; Nylander's Reagent, 357; Pancreatic Solution, 357; Pepsin Solution, 357; Potassium Bichromate N/2, 357; Potassium Permanganate N/20, 357; Potassium Pyroantimonate $\mathrm{K}_{2} \mathrm{H}_{2} \mathrm{Sb}_{2} \mathrm{O}_{7}, 357$; Silver Nitrate (Standard) for Volhard Chloride Method, 357; Sodium Cobaltinitrite $\mathrm{Na}_{3} \mathrm{Co}\left(\mathrm{NO}_{2}\right)_{6}, 357$; Special Sodium Acetate Solution (For Uranium Acetate Method for Phosphates), 358; Stokes' Reagent, 358; Toepfer's Reagent, 358; Uranium Acetate Solution (Standard), 358. 


\section{PHYSIOLOGICAL CHEMISTRY}

\section{PART I}

\section{INTRODUCTORY}

The scientific field known variously as Physiological, Biological or Biochemistry is the branch of science which treats of the chemical constitution, reactions and products of living material, whether of animal or plant origin. There is a growing tendency to use the terms Biological and Biochemistry to denote the entire field, and to restrict the term Physiological chemistry to that portion of the subject dealing with animal material, but this practice is by no means general. It was once believed that the chemical processes going on in plants and animals were fundamentally different. Synthesis or building up was considered characteristic of plants, whereas animals were known to desynthesize or break down the substances which they ate. We now know that this difference is a quantitative anc not a qualitative one, for if kept in the dark, plants take up oxygen, burn their constituents and give off carbon dioxide in a manner analogous to the process predominating in animals. Animals, on the other hand, are now known to be capable of performing numerous and elaborate syntheses, breaking down the materials of their food to simpler compounds, but rebuilding many of the fragments into tissue substance, or altering them to produce compounds having specialized biological functions.

Object and Importance of Physiological Chemistry.-The ultimate object of workers in the field is to establish a rela- 
tionship between chemical composition and biological function, to be able to explain the workings of cells or of the various organs and tissues in terms of chemical reactions, but experimentors are still far from the attainment of this end, although many problems now are clearly understood which a few years ago were still unsolved. The findings of physiological chemists, and the methods of analysis developed by them have been of the greatest value to the science of medicine in general, and to the medical practitioner in particular. Since the body is made up of chemical compounds, and since many of its activities depend upon chemical reactions, it is obvious that any light thrown upon the nature and properties of its components will tend to make clearer the character of the reactions involved in the normal functioning of the tissues, thus furnishing a basis for the study and correction of abnormal or diseased conditions. Both diagnosis and treatment have come to deperid more and more upon the findings of the physiological chemists, and the general advancement of medicine has been greatly furthered by the results of biochemical research.

Protoplasm.-Living material, whether of plant or animal origin has been found to consist of a substance which is strikingly uniform throughout the entire living world. This material has been given the name protoplasm (from the Greek words meaning "first," and "form"). It is a jelly-like watery mass, sometimes fairly rigid in form, possessing certain characteristics which serve to distinguish living from lifeless material. The first of these is the power of growth,growth from internal forces such as we observe in animals and plants, and not growth from without such as the enlarging of a crystal. The second is the power of respiration,--taking up oxygen and giving off carbon dioxide. The third is the power of movement,- from place to place in animals, and movement incident to growth in plants. The fourth is irritability, and the fifth the power of reproduction. All living material possesses these five properties, and no lifeless material possesses all of them. Physiological chemistry may be looked upon as the 
study of the chemistry of protoplasm, its products and the substances which it requires for the continuance of its normal functions.

Material Bases.-Amounts in Body.-In beginning the study of so broad and complicated a field it is difficult to choose a point of attack. The most satisfactory plan will be first to become familiar with the chemical substances out of which living material is made up. The number of these compounds is naturally large, but for convenience they may be classified in five great groups which are given the name of the Base Materials, or Material Bases.

The five groups are as follows:

I. Inorganic materials including water.

II. Carbohydrates.

III. Fats, Phosphatids and related compounds.

Iv. Proteins.

v. Extractives.

Our first task will be to become familiar with the characteristics and properties of the Material Bases, studying group reactions and also the specific properties of important individual compounds, and methods for their detection and estimation. We will then trace the history of the various substances in their passage through the body, considering their fate in the alimentary canal, their subsequent behavior as constituents in the body tissues and fluids, and the final elimination of end products formed by their destruction.

The relative amounts of the different classes of Material Bases in the animal body are somewhat variable. Water and other inorganic materials make up about $65-70 \%$ of the entire body weight of which only $4.5-5 \%$ is ash and the remainder water. Carbohydrates are present only in small quantities, the amount being less than 1\%. Fats and related compounds vary considerably in amount with the general state of the body, since they may be stored away in large quantities. The body contains on the average about $15 \%$. Proteins vary less from 
an absolute standpoint, but relatively the percentage depends upon the amount of fat. The amount in the body averages also about $15 \%$. Extractives, a heterogeneous group of compounds classed together becausc they are water soluble, and belong in none of the other classes, make up less than $1 \%$ of the body weight. This group will receive no further treatment as such, but the substances included in it (urea, creatinine, etc.) will be discussed individually in connection with the tissues or fluids in which they occur. 


\section{CHAPTER I}

\section{PHYSICAL CHEMISTRY IN ITS RELATIONS TO PHYSIOLOGICAL CHEMISTRY}

Importance.-Among the most striking advances in physiological chemistry in recent years are those in which the methods and principles of physical chemistry have been brought to the aid of biochemists. Investigation of the properties of solutions, of dissociation, osmotic pressure, surface tension, of colloidal solutions, adsorption, diffusion, mass action, ionic equilibrium, hydrogen-ion concentration and many other fields have direct bearing on problems connected with the functioning of the cells and tissues. In fact the continuance of cell activity is inseparably bound up with physicochemical phenomena,--absorption, secretion, excretion, growth, muscular contraction and an endless list of other functions of living matter are carried out in conformity with physicochemical laws. The importance of physical chemistry in biochemical work is thus apparent. It is not within the scope of this book to enter into extended discussion of physicochemical fields. However, some of the more important phases of the subject will be presented briefly.

Osmotic Pressure.-It is a well-known fact that a gas exerts a definite pressure, and that this pressure is inversely proportional to the volume. If the volume is halved, the pressure is doubled, etc. The volume of a gas allowed to expand increases $1 / 273$ of its volume at $0^{\circ} \mathrm{C}$. for a rise of $1^{\circ} \mathrm{C}$. Also if an amount of a gas equal in grams to the figure expressing its molecular weight is contained in a 22.4 liter container, it will exert 1 atmosphere pressure. These facts are expressed as the fundamental gas laws.

It has been discovered that dissolved substances also exert a pressure which in many respects behaves in accordance with 
the gas laws. This pressure of a dissolved substance is called osmotic pressure. Obviously it will be impossible to measure this pressure by observing the pressure of the solution on its container. It will be necessary to devise a separating wall or membrane through which the solvent, but not the dissolved substance can pass. Such a membrane is called a semipermeable membrane. A sheet of parchment or a thin film of collodion serves such a purpose. Since the solvent can pass through the membrane, it will tend to pass through the membrane freely. If an apparatus is set up with such a membrane, on one side of which is a solution, on the other pure solvent, the solvent will pass into the solution faster than the reverse process, and tend to dilute the solution. It is possible to place the solution in connection with a manometer, and thus measure the osmotic pressure. Osmotic pressure is directly proportional to the amount of dissolved substance, and to the absolute temperature, at least for dilute solutions. Aside from its usefulness in determining molecular weights and other theoretically important data, it is at once apparent that osmotic pressure will play an enormously important rôle in the body. The body and cell fluids are solutions, and the cell membranes are semipermeable membranes. The maintenance of a proper distribution of the liquids in the body depends on a delicate balance of osmotic equilibrium, as do also such processes as secretion, absorption, ete.

A biological method of measuring osmotic pressure consists in immersing cells in a liquid of known osmotic pressure. If the pressure in the surrounding liquid is higher than that in the cell, water passes out of the cell, and its contents can be seen to shrink away from the cell wall. If the reverse is the case, water passes into the cell, even to the point of rupture. The passage of water through the membrane is called osmosis. The above described method of determining it is called plasmolysis.

If the pores of the membrane are large enough to allow the passage not only of water molecules, but also of simple chemical substances such as salts, but still too small to allow the passage 
of larger molecules such as those of sugar, proteins, etc., the process is called dialysis. Dialysis is of value in freeing from salts, etc., a solution of protein or other nondialyzable substance.

There has been much discussion as to whether osmosis and diffusion or these plus simple filtration could account for the absorption from the intestine, the excretion of urine, the formation of lymph, the passage of gases through the alveolar walls in the lungs and many other processes. Whereas these phenomena doubtless are influenced by the laws governing osmosis and diffusion, they evidently are controlled also by other factors. For example an isolated loop of intestine will absorb substances from within itself which are present in concentration no greater than that in which they exist in the blood, whereas the concentration of many substances in the urine is much above their concentration in the blood. On the other hand, the transference of gases in the lungs probably follows simple laws of diffusion except in time of stress, when cellular activity appears to come into play.

Electrical Properties of Solutions.-It is a well established fact that substances in solution often carry electric charges. This can be demonstrated by passing an electrical current through a solution. The substances in solution will migrate toward the positive or negative pole according as they carry negative or positive charges. This migration in the current is called cataphoresis. Only substances which are dissociated into their ions will migrate thus. Those which are not dissociated do not migrate, and thus do not conduct an electric current. Those which conduct are called electrolytes, those which do not, nonelectrolytes. Knowledge of the electrical properties of dissolved substances has been of the greatest value in the study of colloidal solutions, hydrogen-ion concentration and other important fields.

H-ion Concentration.-It is a well known fact that acids dissolved in water and dissociating into their ions give off hydrogen-ions. The extent to which an acid is dissociated de- 
termines its so-called "strength". Strong acids are much dissociated, giving off much hydrogen ion; weak acids are but slightly dissociated, giving off little hydrogen ion. Even water is a weak acid (and a weak base) since it dissociates thus $\mathrm{H}_{2} \mathrm{O}=\overrightarrow{\mathrm{H}}+\overrightarrow{\mathrm{OH}}$. In water the numbers of $\overrightarrow{\mathrm{H}}$ and $\overline{\mathrm{OH}}$ ions are equal, and such a solution is called neutral. If the hydrogen ions exceed the hydroxyl ions in number, the solution is called acid. If the hydroxyl ions exceed, it is called alkaline.

The extent to which a substance dissociates into its ions can be expressed by a figure called the dissociation constant. For water this is expressed as follows: $\frac{+\mathrm{H} \times \overline{\mathrm{OH}}}{\mathrm{H}_{2} \mathrm{O}}=\mathrm{k}$.

But for water or an aqueous solution the concentration of undissociated $\mathrm{H}_{2} \mathrm{O}$ is so great that it can be regarded as a constant, and the equation thus becomes $\overline{\mathrm{H}} \times \overline{\mathrm{OH}}=\mathrm{Kw}$. $\mathrm{Kw}$ is called the water constant. It has been found that there are $0.000,000,1$ gram ions of hydrogen ions and of hydroxyl ions in 1 liter of water. Expressed with logarithms this figure is $10^{-\tau}$. The above equation then becomes $10^{-7} \times 10^{-7}=\mathrm{Kw}=10^{-14}$. If we measure the concentration either of hydrogen ions or of hydroxyl ions it is thus possible to calculate the other from this equation.

The symbol used to express hydrogen-ion concentration is $\mathrm{C}_{\mathrm{H}}$. It indicates the amount of hydrogen ion in 1 liter of the liquid. Thus the $\mathrm{C}_{\mathrm{H}}$ of water is $0.000,000,1$ or $10^{-7}$. It is obviously inconvenient to use such an expression, so a shorter symbol has been devised, the $\mathrm{pH}$. The $\mathrm{pH}$ is the logarithm of the $\mathrm{C}_{\mathrm{H}}$, with the minus sign omitted. Thus the $\mathrm{pH}$ of water is 7 . If the solution is acid, the amount of hydrogen ion will be greater than $10^{-7}$, say $10^{-6}, 10^{-5}$, etc., and the $\mathrm{pH}$ will be 6,5 , etc. $\mathrm{A} \mathrm{pH}$ smaller than 7 indicates thus that the liquid is acid in reaction, whereas in an alkaline solution the $\mathrm{C}_{\mathrm{H}}$ is less than $10^{-7}$, say $10^{-8}$, $10^{-9}$, etc., and the $\mathrm{pH}$ will be 8,9 , etc., that is, greater than 7 . 
Titratable Acidity.-It often is desirable to know the total amount of an acid or alkali present in a solution, regardless of the extent to which it is dissociated. The standard used in discussing this value is the normal solution. A normal solution of an acid is one which contains per liter one gram equivalent (1.008 grams) of replaceable hydrogen. This hydrogen need not be ionized, but only ionizable. If 36.458 grams of hydrochloric acid is dissolved in water and made up to 1 liter, this will contain 1.008 grams of ionizable hydrogen, and will be a normal solution. Any decimal fraction of this strength also may be prepared as $\frac{\mathrm{N}}{10}(0.1 \mathrm{~N})$, etc. If the acid is sulphuric, one-half the molecular weight will be used, as it will furnish 1.008 grams of replaceable hydrogen. This is more fully discussed, and directions are given for making up normal solutions in the laboratory section. A normal solution of an alkali is one which will neutralize volume for volume a normal solution of an acid.

If the acid were completely ionized, it would be easy to compute the $\mathrm{pH}$ of any normality. Thus, in a $\frac{\mathrm{N}}{10}$ acid there is 0.1 . gram ions of hydrogen per liter. The $\mathrm{C}_{\mathrm{H}}$ is $10^{-1}$ the $\mathrm{pH}$ is 1 . To calculate the $\mathrm{pH}$ of $\mathrm{a} \frac{\mathrm{N}}{10}$ alkali one falls back on the water constant $\stackrel{+}{\mathrm{H}} \times \overline{\mathrm{OH}}=10^{-14}$. If $\mathrm{H}=10^{-1}$ then $\overline{\mathrm{OH}}=\frac{10^{-14}}{10^{-1}}=10^{-13}$. The amount of hydrogen ions in a $\frac{\mathrm{N}}{10}$ sodium hydroxide solution is thus $0.000,000,000,000,1$ or $10^{-13}$ and the $\mathrm{pH}$ is 13 . T'his is evident, for if the equation $\overrightarrow{\mathrm{H}} \times \overline{\mathrm{OH}}=10^{-14}$ holds true for water solutions, an increase in either $\overrightarrow{\mathrm{H}}$ or $\overline{\mathrm{OH}}$ will of course cause a decrease in the other (the figures given for $\frac{\mathrm{N}}{10}$ acid and alkali assume complete dissociation, which really does not 
take place at these concentrations, but the $\mathrm{pH}$ figures given are approximately correct for $\mathrm{HCl}$ and $\mathrm{NaOH}$ and illustrate the point without undue complexity).

A H-ion concentration of 0.035 per liter may be expressed as $3.5 \times 10^{-3}$, etc.

From a biochemical viewpoint the hydrogen-ion concentration is of the very greatest importance. On it depend the action of enzymes, the behavior of colloidal solutions (see later discussion) the proper functioning of cells and tissues and other important processes. The determination of the hydrogen-ion concentration can be made accurately by electrochemical methods, but these are too difficult for the average physician. It is possible to make approximate determinations by the use of substances called indicators. These are compounds of various types which have one color in acid solution, another in alkaline. Their use in titration already should be familiar to the student. Now it happens that the indicators differ in the point of acidity $(\mathrm{pH})$ at which they change color. Some change at the neutral point $(\mathrm{pH}=7)$ others at a $\mathrm{pH}$ of $4,5,6,8,9$, etc. The reason for this is as follows. The common indicators are themselves weak acids. As the free acid they have one color, as their salts another color. This is believed to be due to an internal rearrangement of the molecule when in the salt form. Some indicators are stronger acids than others. If an alkali is added to a mixture of two acids, one strong and the other weak, the strong acid gets the base and forms its salt. If more alkali is added, finally a point is reached at which the weaker acid begins to get some of the base and form its differently colored salt, and a color change occurs. The weaker the acid (the indicator) the more alkali will have to be added before it can get any of the base. Two examples will illustrate this, methyl orange and phenolphthalein. Of these, the former is the stronger acid. When, by the addition of alkali the $\mathrm{pH}$ of an acid solution has been brought to 3 or 4, the methyl orange will get some of the base and change color. The weaker acid, phenolphthalein, will 
not get any of the base until the $\mathrm{pH}$ has been brought to 9 or 10. If one wishes to titrate a weak acid, such as an organic acid, one will choose phenolphthalein as indicator, for the weak acid gives so low a hydrogen-ion concentration that on the addition of alkali the turning point of such an indicator as methyl orange ( $\mathrm{pH} 3$ to 4 ) would be reached long before the acid had been neutralized. On the other hand, if one wishes to titrate a strong acid $(\mathrm{HCl})$ in the presence of a weak one $\left(\mathrm{H}_{2} \mathrm{CO}_{3}\right.$ or an organic acid) it will be possible to do so using methyl orange, for even a small amount of $\mathrm{HCl}$ gives a concentration of $\mathrm{H}$ ions high enough to affect methyl orange, whereas, when the strong acid is all neutralized, the methyl orange changes color even though there is a weaker acid still present untitrated.

Of course such values are not strictly accurate, but they are sufficiently so for many biological purposes.

Also the hydrogen-ion concentration of a solution can be determined by the indicator method. By making up a series of solutions of graduated hydrogen-ion concentration and adding certain indicators, one can get a series of solutions graded in color or depth of hue. Making up an unknown solution under similar conditions and adding the indicator, it is possible to match the hue with that of one of the tubes in the standard series, and thus determine with a fair degree of accuracy the $\mathrm{pH}$ of the unknown.

The student should be careful to get clearly in mind the difference between hydrogen-ion concentration and titratable acidity. The former is a measure of the amount of dissociated or ionized hydrogen per liter; the latter is the amount of replaceable hydrogen, which need not necessarily be ionized. Whereas the titratable acidity of $\frac{\mathrm{N}}{10}$ solutions of hydrochloric and acetic acid is the same, assuming of course the use of an indicator sufficiently sensitive to $H$ ions, the former has a far greater $\mathrm{H}$-ion concentration than the latter, because the former is more highly dissociated. 
Substances called buffers are of great importance in biochemistry. If one adds a small amount of hydrochloric acid to water, the water becomes distinctly acid, and its $\mathrm{pH}$ changes from 7 to some smaller figure. For example 1 liter of water (or say 999 c.c.) $(\mathrm{pH}=7$ ) is acidified with 1 c.e. $0.01 \mathrm{HCl}$ ( $\mathrm{pH}$ about 2). The acid is diluted 1000 times. The $\mathrm{C}_{\mathrm{H}}$ of the solution now is $\frac{0.01}{1000}=.000,01$ and the $\mathrm{pH}=5$. This small amount of dilute acid has caused a marked change in the pH. Suppose that acid were added to a solution of $\frac{\mathrm{N}}{10} \mathrm{NaHCO}_{3}$. If a whole liter of $\frac{\mathrm{N}}{10}$ $\mathrm{HCl}$ were added to a liter of the bicarbonate, the $\mathrm{NaHCO}_{3}$ would be converted into $\mathrm{H}_{2} \mathrm{CO}_{3}$, which is a very weak acid and the solution would still be somewhat near to the neutral point. The $\mathrm{NaHCO}_{3}$, itself forming a solution which is about neutral, still can neutralize strong acids, and the solution remains near the neutral point until the $\mathrm{NaHCO}_{3}$ has been all used up. $\mathrm{Na}_{2} \mathrm{HPO}_{4}$ and $\mathrm{NaH}_{2} \mathrm{PO}_{4}$, proteins, and various other substances behave similarly, and are of the greatest importance as buffers maintaining the reaction of blood and tissues near the neutral point even when acids are produced or absorbed.

Colloidal Solutions.-Certain types of substances when dissolved in water form solutions which differ in many respects from solutions of ordinary chemicals such as hydrochloric acid, sodium chloride, sodium hydroxide, etc. The solutions often are opalescent, they may set to solid jelly like masses, the dissolved substance will not diffuse through a parchment membrane, and they show various other distinguishing reactions. Graham gave the name "colloids". to such substances to distinguish them from substances which form "true solutions." These latter he called crystalloids. Since many of the constituents of living tissues form colloidal solutions the properties of such solutions are of great importance in biology, and have been much studied. It soon became evident that the colloidal state is only a condition in which any substance can exist, if properly prepared, and is not confined to "glue-like" substances (colloidal means 
"like glue"). Substances which usually are colloidal often can be crystallized, whereas crystalloids also can be made to assume eolloidal form. It has become known that colloidal properties are dependent on the fineness of subdivision of the dissolved substance, that is, the size of the particles in the solution. If a more or less coarse insoluble material is shaken up with water, a portion remains suspended. This mixture is called a suspension. If the particles of this suspended material could now be ground up so fine that they no longer could be seen, even with the aid of a microscope, we would have a cloudy liquid, the particles of which would not sink to the bottom on standing: If the particles were sufficiently small, we now would have a colloidal solution. If this process were continued further, we would ultimately arrive at individual molecules. This would then be called a true solution, since the dissolved particles now would be so small that they could pass through the pores of a parchment membrane. Fairly definite limits have been set for the size of colloidal particles. $1 \mu$ is $0.001 \mathrm{~mm}$. and $1 \mu \mu$ is $0.001 \mu$ or one-millionth of a millimeter. The size of colloidal particles has been set as ranging from 1 to $100 \mu \mu$.

In thus subdividing material into such extremely small particles the surface is enormously increased. If a cube of material $1 \mathrm{~cm}$. on edge were divided into cubes $10 \mu \mu$ on edge the surface would be increased from $6 \mathrm{sq}$. cm. to $600 \mathrm{sq}$. meters and there would be $10^{18}$ particles. The peculiar properties of colloidal solutions are thus largely dependent on the enormous increase of surface, and the consequent importance of surface forces, such as surface tension.

Classification and Properties of Colloids.-The colloids are by no means a unified chemical group, for substances of the most widely diverse chemical nature, such as metals, salts, acids, bases, proteins, carbohydrates, etc., may form colloidal solutions. The term "colloidal" refers in fact to a state of matter, and not to a class of compounds. Many substances of the greatest biological importance form colloidal solutions, in fact the constituents of the living cell are believed to be in a col- 
loidal state, so that the properties of colloids are both interesting and important. The group is divided into two classes, hydrophile or emulsoid colloids, and suspensoid colloids. $\mathrm{Hy}$ drophile colloids more elosely approach the crystalloids in their properties, whereas suspensoid colloids are more nearly like suspensions. As a matter of fact there is no sharp dividing line either between the groups, or between emulsoids and true solutions, or suspensoids and suspensions, since all gradations exist. Peptones, although belonging to the class of derived proteins, will pass through a parchment membrane fairly well, and are thus between the emulsoids and crystalloids. Certain metal hydroxides form gels, but are precipitated easily by electrolytes, and are thus between the emulsoids and suspensoids. Kaolin shaken up with water is midway between the suspensoids and true suspensions.

Emulsoid colloids are characterized by the fact that they form gels if sufficiently concentrated, and are not easily precipitated by the addition of salts. If in solution, the substance is called a hydrosol, if in a gel form, a hydrogel. Examples of emulsoids are albumin, gelatine and other proteins, starch, etc. The emulsoid colloids have some attraction for, or relation with the water surrounding them. This property is of the greatest importance, for the colloids of the living protoplasm aid in holding the water which is essential to the life and functioning of living cells.

The suspensoid colloids do not form gels, and are easily precipitated by the addition of even a small amount of a salt. Examples of this class are colloidal metals, sulphides, etc. They seem to have little relation to the water surrounding them.

The particles in colloidal solutions are so small that they will pass through an ordinary filter with ease. Filters impregnated with collodion have been prepared, however, by means of which colloid particles can be held back. In this, and other ways, the size of the particles has been estimated.

Tyndail's Phenomenon.-Colloidal solutions show an interesting behavior known as Tyndall's phenomenon. If a beam 
of light is passed through a colloidal solution, the path of the ray becomes visible, in much the same manner as the path of a ray of sunlight in a dusty room. The light is dispersed or reflected from the particles of the colloid.

An instrument devised on the above principle is known as an ultramicroscope. While the observer looks through a high power microscope at a drop of the solution, a powerful beam of light from an are is passed through the solution from the side. The observer sees tiny flashes of light reflected up from the colloidal particles. Only those of larger size will show this phenomenon, as colloidal gold particles. Colloidal albumin is invisible in the ultramicroscope. The limit of microscopic visibility is about $0.1 \mu$. Particles of this size or larger are called microns. The limit of ultramicroscopic visibility is about $15 \mu \mu$ in electric light or $5 \mu \mu$ in bright sunlight. Particles ranging from 1-100 $\mu \mu$ are called submicrons, and those smaller than $1 \mu \mu$ amicrons. Molecules and ions are much too small to be visible in the ultramicroscope. As seen in the ultramicroscope, colloidal particles appear to be jumping about rapidly. This is known as Brownian movement, and is supposed to be due to the bombardment of the particles by the molecules of the solvent.

Electrical Properties of Colloids.-Colloidal particles carry electrical changes just as ions are electrically charged. This may be demonstrated by passing an electric current through a colloidal solution. The particles of the colloid will move to the positive or negative pole according to the nature of the charge carried,-a colloid with a negative charge travelling to the positive pole, and vice versa. This phenomenon is known as eataphoresis. Whereas some colloidal particles probably have but one electrical charge, undoubtedly they often carry more than one. A protein in colloidal solution will have a positive charge if the solution is acid in reaction, but a negative charge if the solution is alakaline. We may imagine that this is brought about as follows: in acid solution the protein combines with some of the acid, for example hydrochloric acid. From this complex compound, negatively charged chlorine ions are 
given off into the water, and positive charges will remain on the colloid particles. In alkaline solution the protein forms salts, such as the sodium salt. Sodium ions are given off, carrying positive charges, and negative charges will remain on the colloid particles. These facts are of great importance in many of the precipitation reactions of the colloids.

Methods of Precipitating Colloids.-Some colloidal solutions will precipitate, the colloid flocking out, merely on standing. Some will precipitate if they are boiled. Some substances are soluble in hot water, but their solutions will solidify on cooling. Some colloids are thrown out of solution by the addition of an electrolyte. The suspensoid colloids are precipitated by adding a very small amount of an electrolyte such as a salt or an acid, but the emulsoid colloids are precipitated much less readily, that is, only by adding much more of the electrolyte. It has been observed that the effective part of the precipitating salt or substance is the ion bearing the opposite charge to that on the colloid. If the precipitating part of the salt is the metal, then in general colloids bearing negative charges will be precipitated. In this connection it has been observed that trivalent metals are better precipitation reagents than divalent metals, and divalent metals in general are better precipitation reagents than monovalent metals. Thus to precipitate a given colloid from solution a ferric salt is better than a mercuric salt, and a mercuric salt better than a sodium salt. That is, a smaller concentration of ferric chloride than of mercuric chloride is required, etc. But all ions of the same valence do not have equal precipitation powers. They vary according to their solution tension.

When a colloid is precipitated by an electrolyte the precipitate contains some of the precipitating ion, so the precipitate is believed to be a compound of the colloid and the precipitating ion. The precipitation of colloids, however, is undoubtedly dependent on other and more complicated factors than the mere formation of salts or similar compounds of the colloids. For further discussion of this subject the student is referred to larger or more specialized works. 
A suspensoid colloid, as has been stated, is easily precipitated by the addition of an electrolyte. If a small amount of an emulsoid colloid is added to a suspensoid colloid solution, the latter is much less easily precipitated. The suspensoid colloid is "protected" by the emulsoid colloid (albumin, for example). This phenomenon is called the protective action of colloids.

Much interest has centered around gel formation and gel structure. Often a very small amount of material will form a gel holding fairly rigid a relatively enormous volume of water. The jelly like consistency of protoplasm is due to the colloidal character of its constituents, so that gels are of the greatest biological importance. Gels are now considered to be a network of tiny crystal-like formations which hold the water in large measure enmeshed. If a gel stands for some time, the mass of crystals shrinks, squeezing out some of the liquid. This process is called synresis or "bleeding."

Emulsoid colloids enormously increase the viscosity of solutions, suspensoid colloids searcely at all.

Adsorption-Surface T'ension.-Colloidal particles have the property of taking up other substances on their surfaces. This process is called adsorption. It is of great biochemical interest, since it undoubtedly plays a part in many important processes, such as the formation of the temporary union between an enzyme and its substrate, the combination of toxins with antitoxins, the sensitization of leucocytes by opsonins, the taking up of bacteria by leucocytes, the formation of compounds between proteins, lipins and other constituents of the cell.

The process is closely related to surface tension.

At the surface between two media, for example water and air, the molecules of water in the surface layer are attracted downwards and to all sides by their fellows, but not up. The molecules in the surface are thus drawn down, and form what is called the surface film. There is a constant tendency for the amount of free energy in a system to decrease. This is the principle of Willard Gibbs. Now in the surface between two liquids, or a liquid and a solid, the presence of dissolved sub- 
stances tends to decrease the surface tension. Thus, in accordance with Gibbs' principle the dissolved substance will tend to collect or accumulate in the surface film,-to concentrate on the surfaces of the dissolved particles. This is adsorption. Of course the adsorbed substance may combine chemically with the material in colloidal form, and it may be held on by electrical forces. Adsorption phenomena are without doubt of great importance in the body. Much study is being expended upon them at the present time.

Imbibition.- The swelling up of a substance like gelatin when placed in water is called imbibition. The gelatin particle increases greatly in size, but does not 'go into solution. This process is important, as in this way cells take up water. The extent to which imbibition will proceed is greatly influenced by the presence of salts, acids, bases, etc. Salts usually repress the swelling to a point below that which would be reached in pure water. Acids affect it differently according to their concentration. At about $\frac{\mathrm{N}}{320}$ the greatest swelling takes place. If the strength of the acid is less than this, the swelling will be smaller than in pure water. In stronger acid, the swelling is at first rapid, but it does not reach so great a final value as in the concentration specified above.

From the foregoing pages it will be evident that colloidal solutions are of great interest in connection with the study of the chemistry of the body. 


\section{CHAPTER II}

\section{ELEMENTS, INORGANIC MATERIALS, WATER}

Elements Found in the Body.-The body is made up of a large number of chemical elements which are present in very unequal amounts, and distributed quite unevenly in the various tissues and body fluids. Certain of these elements are in all living cells, others only in particular kinds of cells or in particular animals. Still others are present only accidentally or temporarily. The elements found most frequently are the non-metals earbon, hydrogen, oxygen, nitrogen, sulphur, and phosphorus; the metals sodium, potassium, calcium, magnesium and iron; the halogens, chlorine, iodine, and fluorine. In addition, there are many other elements such as silicon, copper, manganese, arsenic, silver, lead, bromine, lithium, etc., found only in traces or only in a few animals.

Importance Not Determined by Amount Present.-Three of the elements, carbon, hydrogen and oxygen alone make up over $90 \%$ of the body weight. The conclusion should not be drawn, however, that these are the only important elements and that those elements or compounds present in small amounts or traces are relatively unimportant to the organism. Quite the contrary may be the case. The body of an average-sized adult man contains only about three grams of iron, and yet this is so necessary to life that an animal fed for some time on a diet which contains no iron will die quite as surely, though of course not as quickly, as if it had been deprived of food altogether. The principle involved may be formulated as the Law of the Minimum which states that the importance of a given substance to the animal organism is independent of the relative amount in which it is required. Striking examples of this principle have developed in recent years, and we now know that the body requires eertain compounds of which nothing 
was known a few years ago. Some of these substances appear to be organic compounds. Although the amounts of these materials of unknown constitution which are required by the body are only a small fraction of a gram, still their absence from the diet will cause severe disorders and ultimate death.

The importance to the body of the individual elements extends much beyond the function of serving as inert building stones out of which the body materials are made up. Many of the chemical reactions in the body are greatly influenced or modified by certain of the elements. For example, two important processes, the clotting of the blood which tends to protect a wounded animal from bleeding to death, and the clotting of milk in the stomach, the first step in its digestion, are dependent upon the presence, among other things, of calcium, and without this metal neither of these reactions can occur.

The accompanying table gives a survey in round numbers of the relative amounts of the elements present in the body.

Carbon .................... 17.5

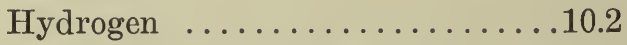

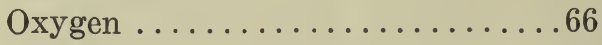

Nitrogen ................. 2.4

Sulphur .................... 0.2

Phosphorus ................ 0.9

Sodium .................. 0.3

Potassium ................ 0.4

Calcium ............... 1.6

Magnesium ................ 0.05

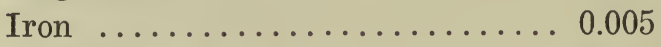

Chlorine ................. 0.3

Iodine .................. Traces

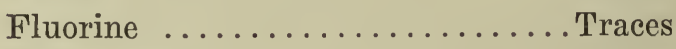

Other elements .............Traces

Carbon, Hydrogen, Oxygen, Nitrogen, Sulphur and Phosphorus.-Carbon has the property of forming a very large number of compounds with hydrogen, oxygen, and sometimes sul- 
phur, phosphorus and other elements. These compounds are so numerous that a separate branch of chemistry is devoted to them under the name of organic chemistry. Thirty per cent or more of the body consists of organic material, or compounds of carbon with hydrogen, oxygen, etc. The importance of these compounds is so great that special chapters will be devoted to the important groups. The body receives its carbon compounds in the foods, the solid portion of which is very largely organic material. Aside from the organic components of the tissues, carbon is also found in inorganic form, chiefly as calcium carbonate in bone, and as sodium bicarbonate in the blood. This latter compound is of great importance, since, although its solution is neutral, it has the power of neutralizing acids, thus protecting the tissues when acids are introduced into the blood either from the digestive tract or as the result of the breaking down of body materials. Carbon is eliminated from the body as carbon dioxide, given off through the lungs, or as more complex compounds such as urea and other materials excreted in the urine or the feces. If heated, organic compounds char, leaving a black residue of carbon.

Hydrogen and oxygen aside from being constituents of water, organic and inorganic compounds, also play more specialized rôles in the body. Oxygen, which makes up about two-thirds of the entire body weight is taken up as a constituent of the compounds in the food and in the process of respiration. $\mathrm{Hy}$ drogen, the presence of which in ionic form is the distinguishing characteristic of acids, plays an important part in the digestion of proteins by gastric juice. Hydrogen and oxygen are eliminated from the body chiefly as water and $\mathrm{CO}_{2}$ given off through the lungs, and as water and in organic compounds in the urine and feces. On heating organic materials they decompose, and the hydrogen and oxygen are given off as water and various other compounds.

Nitrogen, sulphur and phosphorus also play various rôles in the body and all three are found both in organic and inorganic compounds. Nitrogen is a constituent of all proteins. Many 
proteins contain sulphur and phosphorus as well. Nitrogen occurs in various other compounds throughout the body, and the body fluids contain gaseous nitrogen in solution, as is to be expected since nitrogen is somewhat soluble in an aqueous liquid, and in the lungs the blood comes in contact with the air which is made up about four-fifths of nitrogen. So far as is known this dissolved nitrogen has no influence on the body's activities. Nitrogen is excreted mainly as urea, uric acid and other products formed by the decomposition of proteins in the body. If heated with soda lime, an organic substance of this type gives off its nitrogen as ammonia. Sulphur and phosphorus are present in various compounds other than the proteins. Of these the phosphatids are perhaps most interesting. This group will be considered later. Calcium phosphate makes up about $85 \%$ of the ash of bone. Sodium phosphate is found in the blood and tissues. Sulphur and phosphorus are excreted mainly in the urine as sulphates and phosphates, but also in the feces. Unoxidized sulphur may be split off from organic compounds by boiling with alkali. On adding lead acetate, a dark precipitate of lead sulphide will form. This test may be confirmed by adding an acid. Hydrogen sulphide will be given off and may be identified by a paper moistened with lead acetate on which lead sulphide will form as a shiny dark precipitate. Sulphates are detected by precipitating as barium sulphate. Phosphates are detected by precipitating as ammonium phosphomolybdate.

Sodium, Potassium, Calcium, Magnesium and Iron.-These metals make up only a small part of the body, but they are none the less important. They are distributed widely throughout the body tissues and fluids. There usually is more sodium than potassium in body fluids, and more potassium than sodium in the solid tissues. These metals are present as chlorides, sulphates or phosphates. Sodium chloride in the blood is interesting since it furnishes chlorine for the hydrochloric acid of the gastric juice, whereas sodium bicarbonate is very important in preserving the neutrality of the blood as above noted. Sodium 
chloride is the only inorganic substance which is contained in a mixed diet in amounts insufficient for the body's needs. Accordingly our food must be salted. Herbivorous animals, living on plants in which potassium is in excess of sodium, crave salt, and it is well known that cattle must be "salted" to keep them in good health. Sodium and potassium also affect the irritability of muscle and nerve tissue so that their rôle in the organism may perhaps include little understood regulatory functions. These metals may be detected by the flame test or by precipitation as sodium pyroantimonate or potassium cobaltinitrite.

Calcium and magnesium, while found in all cells in the body, are present in largest amount in the bones and teeth, which contain about $99 \%$ of all the calcium and $70 \%$ of all the magnesium in the body. Their phosphates and carbonates make up about $98.5 \%$ of all the inorganic material in bone. That these metals have other rôles to play in the body, however, is demonstrated by the failure of blood and milk to clot in the absence of calcium. Calcium is detected by precipitation as calcium oxalate. Magnesium is present in much smaller amounts than calcium, and less is known of its physiological activities. It is interesting in this connection that magnesium sulphate acts as an anæsthetic on mammals and that it paralyzes the endings of the motor nerves in the muscles. Magnesium is detected by precitation as magnesium ammonium phosphate.

Iron, though present only to the amount of a few grams in the body of an adult man, still is distributed very widely. Its most conspicuous rôle is in connection with the hemoglobin of the blood, of which it is a constituent. This substance carries oxygen from the lungs to the tissues. Iron probably is present also in traces of inorganic iron compounds. The spleen contains relatively much, so that it has been suggested that the spleen has some rôle to play in connection with the iron compounds of the blood. Also the liver is concerned with the fate of hemoglobin, and serves as a clearing house through which hemoglobin from worn out corpuscles is broken down and excreted by way of the bile into the intestine. Iron is also ex- 
creted in the urine, the amount being no greater than 10 or 11 mg. per liter.

Iron is detected by precipitation as "Prussian Blue" or ferric ferrocyanide or by the formation of ferric thiocyanate which is red in color.

Chlorine Iodine, Fluorine, etc.-Chlorides are present in traces in all the tissues and fluids; the blood contains $0.5-0.6 \%$ sodium chloride, the gastric juice about $0.4 \%$ hydrochloric acid. The necessity of adding sodium chloride to the diet has already been referred to. Chlorides are excreted in the urine in amounts varying with the amount in the food, 10-12 gms. daily being an average figure. Chlorine may be detected by precipitation as silver chloride. Iodine is interesting chiefly in connection with its occurrence in the thyroid gland. This gland, by means of a compound which it produces and pours out into the blood stream, has far reaching influence on the chemical reactions going on in the body. This important substance is an iodine compound. If an iodide gets into the blood stream, the salivary glands have the power of excreting it. After taking a capsule containing potassium iodide, iodine may be demonstrated in the saliva. Fluorine is found in the bones and teeth.

Other elements may occur either regularly or accidentally in the body tissues or fluids, but usually only in traces.

Water.-Water makes up about $2 / 3$ of the body weight of mammals, and a much larger part in some lower animals. All tissues contain it, even the enamel of the teeth: blood, lymph, the digestive juices, urine, etc., are $8 / 10^{-9} / 10$ water; the organs and softer tissues about $3 / 4$. Water serves a variety of functions. It is the circulating medium for transporting food and waste material to and from the cells, it holds various substances in solution and makes ionization possible, and is a medium for the excretion of waste. It also distributes the heat of the body, and by evaporation from the skin is instrumental in regulating body temperature. An animal may survive for many days without nourishment, but if water also is withheld, death follows in a few days' time. 


\section{CHAPTER III}

\section{CARBOHYDRATES}

Composition, Occurrence, General Function.-The carbohydrates are compounds of carbon, hydrogen and oxygen in which the hydrogen and oxygen are present usually in the same proportions as in. water, hence the name of the group. A few carbohydrates do not conform to this general statement, for example Rhamnose $\mathrm{C}_{6} \mathrm{H}_{12} \mathrm{O}_{5}$. Some substances not carbohydrates do contain hydrogen and oxygen in this proportion, such as acetic acid $\mathrm{C}_{2} \mathrm{H}_{4} \mathrm{O}_{2}$ and lactic acid $\mathrm{C}_{3} \mathrm{H}_{6} \mathrm{O}_{3}$. Thus, this characteristic is not distinctive of the group. Carbohydrates vary considerably in their properties. They are found both in plants and in animals, but chiefly in the former, in which they form a considerable part of the structural frame work. In animals they serve mainly as a fuel to be oxidized for the production of heat or the performance of mechanical work, but they may be laid away as a reserve store to be called on in case of need.

Structure of the Carbohydrates.-The carbon atoms in the carbohydrate molecule are linked together in a long chain. It has been shown that the molecule contains several hydroxyl groups, and that in the simpler members of the group at least

there is either an aldehyde $-\stackrel{C}{C}=0$ or a ketone $\underset{\mid}{\mathrm{C}}=0$ group.

The remaining valencies of the carbon atoms in the chain hold the hydrogen atoms not accounted for. Thus the formula for glucose, one of the most important carbohydrates, has been shown to be as follows: 
<smiles>O=CC(O)C(O)C(O)C(O)CO</smiles>

An example of a sugar containing a ketone group is fructose, which has the following formula:<smiles>O=C(CO)C(O)C(O)C(O)CO</smiles>

Fructose

Many of the reactions characteristic of the carbohydrates depend upon the properties of the aldehyde or ketone groups which they contain. In the case of the more complex carbohydrates, these reactive groupings are usually combined in such a way that they do not show their characteristic behavior.

Optical Activity.-On inspecting the above formulæ for glucose and fructose it will be observed that the hydrogen and hydroxyl groups of the four carbon atoms occupying the middle portion of the chain are not arranged in a regular manner. 
This irregular arrangement is intended to indicate that there is actually a variation in the arrangement of these groups around the carbon atoms in space. This arrangement is a determining factor in the property of these substances known as optical activity. Optical activity is the property possessed by many compounds of rotating the plane of polarized light.

If a ray of white light is passed through a crystal of iceland spar it is split into two slightly diverging rays, but this is not the only change which is produced in the ray. Light is due to vibration of the particles of the ether, the vibration being at right angles to the direction of the ray, and in all possible planes passing through the path of the ray as an axis. We can picture this perhaps by imagining the cross-section of a ray of light to resemble the cross-section of an orange, only with many more planes of vibration. After passing through the crystal of iceland spar, the light is so altered that in each of the two emerging rays vibration is taking place in only one direction. To light of this character is given the name plane polarized light. It has been found that optically active substances have the property, if such a ray of light is passed through their solutions, of rotating the plane in which the ether particles are vibrating, some substances rotating the plane of vibration to the right, others to the left. Not only do these compounds possess this property, but a given substance, under similar conditions of observation (concentration, temperature, etc.) always rotates the plane of polarized light through the same angle. This uniformity of behavior on the part of each optically active substance gives us a most useful means of detecting the presence of the compound in question.

In order to have a uniform standard for comparing observations of optical activity it is necessary to adopt some system for reporting such data. A value has been selected and given the name "Specific Rotation," which is the rotation produced by 1 gram of substance dissolved in 1 cubic centimeter of solvent and the rotation observed in a tube 1 decimeter in length. This value is designated by the symbol $[\propto]$. 
It is obvious that it often will be impossible to observe the rotation produced by a substance under these standard conditions. Many substances are not sufficiently soluble to dissolve 1 gram in 1 cubic centimeter of solvent. To avoid this difficulty, a formula has been developed for use under general laboratory conditions. Let $\propto$ be the observed rotation of a solution under question. Under standard conditions $[\propto]=\propto$. Let $\mathrm{g}=$ grams substance per c.c. of solvent. If $\mathrm{g}$ is not equal to 1 , we can easily find the value of $[\propto]$ by dividing $\propto$ by $\mathrm{g} .[\propto]=\frac{\propto}{\mathrm{g}}$. We must also introduce the length of the observation tube, as naturally a column of liquid longer or shorter than the specified 1 decimeter will give respectively a greater or a smaller rotation. Our formula will now be $[\propto]=\frac{\propto}{\mathrm{l} . \mathrm{g}}$

As it seldom is convenient to work with 1 c.c. of solution, it will be advisable to revise our formula for use with solutions calculated on the basis of 100 c.c. Let c equal the number of grams substance in 100 c.c. solvent, then $\mathrm{g}=\frac{\mathrm{c}}{100 \text {. Substituting }}$ this value of $\mathrm{g}$ in the above equation we have

$$
[\propto]=\frac{100 . \propto}{\text { l.c }}
$$

Now the amount of rotation which a given solution will produce is influenced by various factors, among them the temperature of the solution, the color of the light used in the observation, etc. Unless there are special reasons for other procedures, it is customary to make observations at $20^{\circ} \mathrm{C}$. and with sodium light, corresponding to the $\mathrm{D}$ line in the spectrum. These two influencing factors also are included in our formula, which we now have in its final form.

$$
[\propto]_{\mathrm{D}}^{20^{\circ}}=\frac{100 . \propto}{\text { l.c }}
$$

This value is called the specific rotation of a compound and is a constant for each optically active substance. The specific rotations of most of the sugars have been determined, and may 
be found in textbooks or books of reference. By observing the rotation $\propto$ of a solution of a known sugar and substituting this value in the above equation it is possible to calculate the amount of the given sugar present. On the other hand, if we have a solution containing a known amount of an unknown sugar, it is possible to calculate its specific rotation, which usually will identify the substance, especially if used in conjunction with other tests. For this purpose we may solve our formula for $c$

$$
\mathrm{c}=\frac{100 . \propto}{1 .[\propto] 20^{\circ}}
$$

$\mathrm{D}$

The actual observation of $\propto$ is made with a polariscope, an instrument in which light is polarized and passed through the solution to be examined. The apparatus is so constructed that it is possible to measure the amount of rotation produced by the solution. The accompanying diagram indicates the structure of a Laurent polariscope.

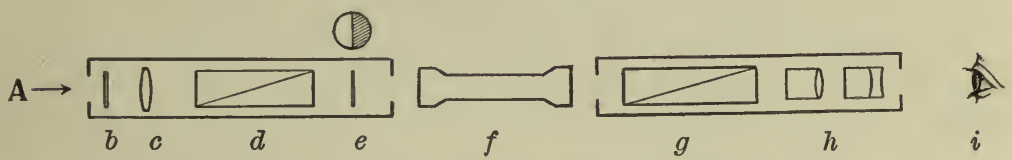

Fig. 1.-Diagram of Laurent Polariscope.

A. Source of light,-sodium flame.

B. Plate cut from a crystal of potassium bichromate. In place of this, a flat-sided cell containing a solution of potassium bichromate may be used to insure absence of other than yellow light.

C. Lens to make the rays of light parallel.

D. Nicol prism, called the polarizer, which polarizes the ray.

E. Quartz plate covering a portion of the field to produce half shadow

F. Tube containing solution to be studied.

G. Second Nicol prism called the analyzer.

H. Lenses for focusing.

I. Eye of observer.

The Nicol prism is a device for polarizing light, and getting rid of one of the two rays into which the original ray is split. A crystal of calcite is sawed through diagonally, and the two pieces stuck together with a thin layer of Canada Balsam.

On entering the prism, light is polarized and split into two diverging rays. From the diagonal surface, one of the two polarized rays is reflected to the side, the other passes on through 
the apparatus. If the tube $f$ contains only water, the polarized ray passes through it without altering the direction of its vibration. On arriving at the second Nicol prism $g$ the ray will pass through unaltered provided the prism is in a position corresponding to that of the first prism. If, on the other hand, the prism $g$ has been rotated to the right or the left so that its position no longer corresponds to that of $d$, only a portion of the ray will pass through, and the intensity of the ray s'aching the eye at $i$ will be diminished. If the prism $g$ is rotated $90^{\circ}$ from the position corresponding to that of $d$, no light will pass through. Beyond this point the illumination increases until at $180^{\circ}$ it again is at its maximum. At $270^{\circ}$ the field again is dark.

Imagine the apparatus set with the two Nicol prisms in corresponding positions. Light will pass through to the eye. Now if a tube of sugar solution is placed at $f$, the ray of light passing from $d$ will be rotated about its axis by the sugar solution, which is optically active. The result will be that it strikes $g$ in a position in which it will not pass through without losing some of its intensity. If we rotate prism $g$ through the same angle through which the ray of light has been rotated by the sugar solution, the ray again will pass through at maximum intensity. By observing the angle through which the prism must be rotated to bring this about, the angle of rotation of the ray caused by the sugar solution is determined.

In practice it would be difficult to observe a changing illuminated field and select the point at which the illumination was at its maximum. A mechanism has been devised to obviate this difficulty. This is represented in the diagram by $e$ which is a thin quartz plate covering one-half the visible field. This plate so alters the light passing through it that when the half of the field not covered by the plate is at its maximum illumination, the half of the field covered by the plate is somewhat darker, and vice versa. By rotating the prism $g$ a point can be found intermediate between the two, at which the two halves of the field are equally light. This is taken then as the 
starting (or zero) point of an observation. When the sugar solution has been introduced the prism $g$ is rotated until the two halves of the field again are equally light, and the reading taken. This will correspond to the rotation produced in the polarization plane of the ray by the sugar.

The readings are made upon a circular scale which usually is graduated in degrees, and provided with a vernier to make it possible to read to minutes.

The rotation produced by a given substance varies with the solvent. Water is the solvent most frequently used. If more than one optically active substance is present in the same solution, account must be taken of this fact, or one or the other removed. Certain sugars when first dissolved in water show a much higher or much lower rotation than after standing for twenty-four hours or so. This is believed to be due to the fact that these substances exist in two forms, of which one possesses much stronger rotating power than the other. The two forms pass into one another spontaneously, and reach an equilibrium in which there is a definite amount of each, the rotation produced by this final mixture being the resultant of the rotations of the two forms present. To this phenomenon is given the name Mutarotation (from the Latin word meaning "to change"). Some sugars show about twice the final rotation when first dissolved, e.g., glucose, and for such cases the term birotation may be employed. Half rotation is the term employed for those substances showing half the final rotation when first dissolved. Instead of allowing a solution to stand until equilibrium is reached, this may be accomplished at once by adding a small amount of alkali to the solution.

The exact reason why some compounds have the property of rotating the plane of polarized light is unknown. This property has been shown to depend, however, upon the presence of what has been called an asymmetric carbon atom, which is a carbon atom united to four dissimilar chemical groups. If the structural formula for glucose is inspected, it will be seen that in the case of four of the carbon atoms in the chain each is united 
to four different chemical groupings. Each of these four carbon atoms is thus asymmetric, and confers the property of optical activity upon the compound. Around each asymmetric atom either of two groupings may exist, one of which rotates the plane of polarized light to the right (dextro-rotatory), the other an equal distance to the left (levo-rotatory). These two compounds are called optical isomers. It has been found that the number of optical isomers possible when a compound contains more than one asymmetric carbon atom, is represented by the value of $2^{n}$ where $n$ is the number of such carbon atoms. We will thus expect to find $2^{4}=16$ different sugars, all isomers of glucose. Twelve of these sixteen actually have been prepared.

Classification of Carbohydrates.-The carbohydrates are divided into three great classes.

These are subdivided as indicated below.

Monosaccharides

Bioses

Trioses

Tetroses

Pentoses-arabinose, xylose, ribose

Hexoses-glucose, fructose, galactose, mannose

Heptoses

Octoses

Nonoses

Disaccharides

Saccharose (sucrose, or cane sugar)

Maltose (malt sugar)

Lactose (milk sugar)

Polysaccharides

Dextrins

Starches

Glycogen

Inulin

Cellulose

Gums and Mucilages. 
The monosaccharides are sometimes called the simple sugars. The disaccharides are so called because they are formed by the union of two molecules of monosaccharide, with elimination of water. Polysaccharides are composed of several molecules of monosaccharide united in a similar manner. The monosaccharides are subdivided into groups according to the number of carbon atoms in the molecule, thus the bioses are sugars having only two carbon atoms in each molecule, the trioses, three, etc. The first six of these groups are found in nature. The octoses and nonoses have been built up in the laboratory. Of the monosaccharides, the pentoses and hexoses are the most important. With the exception of the bioses, there are two classes of sugars in each group,-aldehyde sugars and ketone sugars. Of the biologically important sugars all are aldehyde sugars, or aldoses, with the exception of fructose, which is a ketone sugar or ketose. The individual carbohydrates will be discussed later.

Origin and Synthesis.-The ultimate dependence of the animal world upon plants is well illustrated by the carbohydrates. These compounds which are an important fuel for the body are obtained from plants, in which they are built up from the very simple substances carbon dioxide and water. The exact mechanism by which the plant brings about this important synthesis is a matter of some uncertainty, but it is probable that the carbon dioxide from the air is first reduced to formaldehyde. Several molecules of formaldehyde then condense to form a carbohydrate. The reaction might be represented as follows:

$$
\begin{aligned}
& \mathrm{CO}_{2}+\mathrm{H}_{2} \mathrm{O} \rightarrow \mathrm{H}-\mathrm{CHO}+\mathrm{O}_{2} \\
& 6 \mathrm{HCHO} \rightarrow \mathrm{C}_{6} \mathrm{H}_{12} \mathrm{O}_{6}
\end{aligned}
$$

The energy for this synthesis is derived from sunlight by the agency of chlorophyl, the green coloring matter of plants. The hexose so formed may then be built up into more complex substances such as starch, which is laid away as reserve food in the seeds, tubers and other parts of the plant. Sunlight is not necessary for the second part of this process, as starch can be built up in the roots and tubers, which are underground. By 
variations of this general process the plant undoubtedly can build up a large number of other compounds of the most diverse nature.

The animal body has much more limited powers of synthesizing carbohydrates, although we now know that it is capable of doing much more in this respect than was once thought. The polysaccharide glycogen is regularly built up in the animal body from monosaccharides, and it has been shown in diseases where carbohydrates are lost from the body in the urine, that certain compounds other than carbohydrates apparently can be converted into sugar in the body.

There are various methods for synthesizing carbohydrates in the laboratory. One of these suggests the synthesis in plants. If a solution of formaldehyde is made slightly alkaline, a condensation takes place, and the liquid will be found to contain a hexose, acrose.

A method which has proved very useful in studying the carbohydrates is known as the cyanhydrin synthesis. This serves to lengthen the carbon chain by one carbon atom. Starting from a pentose, a hexose may be prepared, from a hexose a heptose, and so on. The steps of the synthesis are as follows:

$\mathrm{CH}_{2} \mathrm{OH}-\mathrm{CHOH}-\mathrm{CHOH}-\mathrm{CHOH}-\mathrm{CHO}+\mathrm{HCN} \rightarrow$ $\mathrm{CH}_{2} \mathrm{OH}-\mathrm{CHOH}-\mathrm{CHOH}-\mathrm{CHOH}-\mathrm{CHOH}-\mathrm{CN}$

This nitril, containing six carbon atoms is easily saponified.

$\mathrm{HOH}$

$\mathrm{CH}_{2} \mathrm{OH}-\mathrm{CHOH}-\mathrm{CHOH}-\mathrm{CHOH}-\mathrm{CHOH}-\mathrm{CN}+\mathrm{HOH} \rightarrow$ $\mathrm{HOH}$
$\mathrm{CH}_{2} \mathrm{OH}-\mathrm{CHOH}-\mathrm{CHOH}-\mathrm{CHOH}-\mathrm{CHOH}-\mathrm{COOH}+$ $\mathrm{NH}_{3}+\mathrm{H}_{2} \mathrm{O}$

Converting this into its lactone by the action of acid we get:

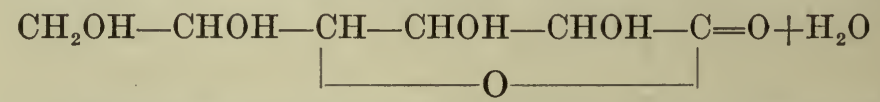

On reducing this with sodium amalgam the compound takes 
up $2 \mathrm{H}$ forming an aldehyde, a hexose, which thus has been built up from a pentose.

\section{$\mathrm{CH}_{2} \mathrm{OH}-\mathrm{CHOH}-\mathrm{CHOH}-\mathrm{CHOH}-\mathrm{CHOH}-\mathrm{CHO}$}

The carbohydrates also may be "built down" step by step in the laboratory. This method also has been of value in studying their constitution. If treated with hydrogen peroxide in the presence of a ferric salt as catalyzer, the elements of formic acid are split off, leaving a carbohydrate with one less carbon atom. Carbohydrates also may be built down by electrolysis or by way of their oximes.

Interconversion of Carbohydrates.-Since the different monosaccharides are closely related compounds, differing only in the arrangement of their hydrogen and hydroxyl groups around the carbon atoms, it is not surprising to learn that certain of them may very easily be converted into one another. Thus if a solution of glucose is made slightly alkaline and allowed to stand for some time, it will be found to contain not glucose alone, but also fructose and mannose. A portion of the glucose is transformed into the other two sugars. It is immaterial which of the three sugars is taken to start with,- the result will be the same and all three will be found in the solution. The interconversion of the simple sugars is of interest physiologically, for in the body such transformations are known to take place. The lactose of milk is produced in the mammary gland from glucose in the blood. Lactose is made up of equal parts of glucose and galactose. A portion of the glucose from the blood thus must be transformed into galactose in the gland. A second instance of a similar transformation is the formation of the polysaccharide glycogen which is stored up in the liver and muscles as a reserve material. On hydrolysis glycogen yields always glucose. It is well known, however, that glycogen will be deposited in the body if an animal is fed fructose or various other sugars. The glycogen formed under these circumstances also yields glucose on hydrolysis, indicating the transformation of the fructose, etc., into glucose. 
Combination of Carbohydrates With One Another, and With Other Substances.-The carbohydrates have the property of combining with various substances including themselves. The union of two molecules of monosaccharide forms the disaccharides, of many molecules of monosaccharide, the polysaccharides. Compounds of monosaccharides with other classes of materials are variously named, and include substances of great biological interest and importance. Examples are the glucosides, - compounds of glucose or one of its derivatives, among which are many of the drugs used in medicine, and some of the constituents of brain and nerve tissue. The term "Glucosides" is sometimes extended to include similar compounds which yield other sugars, e.g., galactose.

Behavior With Strong Alkalies.-The action of weak alkali upon the carbohydrates already has been discussed under the heading interconversion of carbohydrates. A rearrangement of groups in the molecule is observed. The action of strong alkali is much more vigorous and far reaching and the nature of the products formed depends upon the experimental conditions. The alkali undoubtedly combines with the sugar at first. There follows loss of water from groups around neighboring carbon atoms, and a double bond is formed. The carbon chain is then broken, and two compounds are formed, each with a smaller number of carbon atoms than the original substance. The nature of these fragments is only imperfectly understood, but it may be inferred from the final products of the reaction. In ease oxygen is supplied plentifully, the fragments are oxidized to the corresponding acids. Of these a long list may be obtained varying in complexity from formic $\mathrm{H} . \mathrm{COOH}$ and oxalic acids

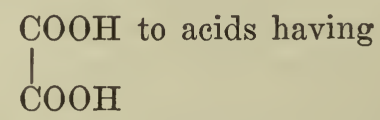

chains as long or only slightly shorter than the chain in the original substance. Evidently the breaking up into fragments may take place at various points in the chain, giving compounds 
containing one, two, three, four, or five carbon atoms. The chain also may remain unbroken.

This process is of biological interest for probably it has many points of similarity with the breaking down of carbohydrates in the body. Of course the body tissues are not strongly alkaline, so that the agent bringing about the change must be some other substance. In the body the fragments thus produced may be used to construct new substances, or they may be further broken down and oxidized to carbon dioxide.

In case the strong alkali acts upon glucose when the oxygen supply is limited, as would be the case if no air were bubbled through the liquid, the reactive fragments produced will combine with one another, forming complex brown substances of a resinous nature, a mixture of which is known as caramel. Possibly this process is analogous to the building up of materials from carbohydrate fragments in the interior of the body cells, although in this latter case the conditions of synthesis are carefully controlled by agents in the cell so that particular substances result which are required by the cell.

Behavior With Acids.-On boiling with dilute acids the complex carbohydrates take up water and are split into simpler substances, ultimately the monosaccharides. If the monosaccharides are boiled with strong hydrochloric acid they are decomposed and yield a variety of products. The pentoses lose water and form furfurol.
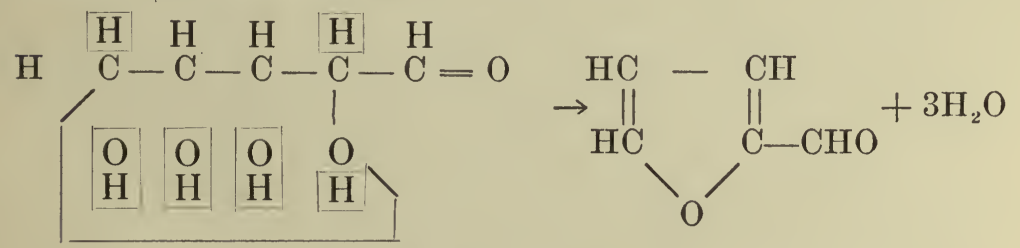

The formation of this compound serves to identify the pentoses, since it forms with various phenols colored substances which may be identified easily. The hexoses, on boiling with concentrated hydrochloric acid yield among other things oxymethyl furfurol, but in much larger quantities levulinic acid 
$\mathrm{CH}_{3} \cdot \underset{\mathrm{O}}{\mathrm{C}}-\mathrm{CH}_{2} \cdot \mathrm{CH}_{2} \cdot \mathrm{COOH}$

Oxidation of Carbohydrates.-Oxidation Tests.-Since the simple carbohydrates contain either an aldehyde or a ketone group they are easily oxidized, even by mild oxidizing agents. The products of mild oxidation are acids having the same number of carbon atoms as the original material. If the oxidation is more vigorous the molecule may break into fragments as was described under the action of alkalies. Some of the most important carbohydrate tests depend upon oxidation processes. Among the oxidation tests are the Fehling, Benedict, Almén-Nylander, and Barfoed tests.

Fehling Test.--If solutions of copper sulphate and sodium hydroxide are mixed, a light blue or whitish precipitate is formed. This is cupric hydroxide

$$
\mathrm{CuSO}_{4}+2 \mathrm{NaOH} \rightarrow \mathrm{Cu}(\mathrm{OH})_{2}+\mathrm{Na}_{2} \mathrm{SO}_{4}
$$

If this mixture is boiled, the precipitate is converted into black cupric oxide.

$$
\mathrm{Cu}(\mathrm{OH})_{2} \rightarrow \mathrm{CuO}+\mathrm{H}_{2} \mathrm{O} \text {. }
$$

If a sugar is present in the solution, however, the copper is reduced by the sugar which is converted into an acid. If a sugar is added to a mixture of copper sulphate and sodium hydrate the liquid will turn a very deep blue, and a much smaller precipitate will form as most of the $\mathrm{Cu}(\mathrm{OH})_{2}$ is held in solution by combining with the sugar. If the mixture is allowed to stand, or if it is boiled, the color becomes perhaps yellow at first, and ultimately a red precipitate forms. The sugar has reduced the cupric hydroxide to yellow cuprous hydroxide, which, on boiling, decomposes into the red cuprous oxide.

$2 \mathrm{Cu}(\mathrm{OH})_{2}+\mathrm{C}_{5} \mathrm{H}_{11} \mathrm{O}_{5} . \mathrm{CHO} \rightarrow 2 \mathrm{CuOH}+$

$$
\mathrm{C}_{5} \mathrm{H}_{11} \mathrm{O}_{5} \cdot \mathrm{COOH}+\mathrm{H}_{2} \mathrm{O}
$$

$2 \mathrm{CuOH} \rightarrow \mathrm{Cu}_{2} \mathrm{O}+\mathrm{H}_{2} \mathrm{O}$

As a matter of fact, the sugar undoubtedly undergoes further change, as we already have seen in considering the action of al- 
kalies on the carbohydrates, but the above equations serve to show the nature of the. part played by the copper compound in the reaction.

The.above test would work very well if the solution to be tested contained much sugar. If this were not the case, however, much black cupric oxide would be formed which might easily obscure any small amount of red cuprous oxide resulting from the reducing action of a small amount of sugar. Accordingly it is more satisfactory to use Fehling's solution for the test. This is made up in two parts, $A$ and $B$, which are mixed in equal quantities immediately before using. $A$ contains copper sulphate; $B$ contains sodium hydrate and sodium potassium tartrate. On mixing these two solutions a deep blue liquid results. The two solutions are kept separate, as otherwise the tartrate will slowly reduce the copper. The advantage in Fehling's reagent lies in the fact that the sodium potassium tartrate unites with the cupric hydroxide to form a complex ion; thus the cupric hydroxide does not precipitate and does not decompose into the black cupric oxide. On boiling, the liquid remains clear and blue. The combined cupric hydroxide is in equilibrium with a very small amount of this compound in solution so that as fast as the free cupric hydroxide is reduced by the sugar solution, more of the copper-hydrate-tartrate compound dissociates. This complex compound thus furnishes a ready supply of copper hydroxide, and if sufficient sugar is present, all of the copper will be reduced. At this point the blue color will have disappeared from the liquid. The following equation illustrates the formation of the complex compound:
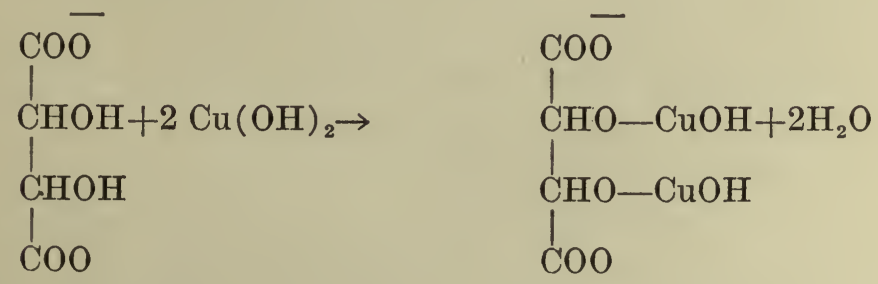
The Fehling test will detect $0.1 \%$ glucose in a solution. Benedict's Test.-The fundamental principle of this test is similar to that of Fehling's test. In place of sodium hydroxide, sodium carbonate is used, and in place of sodium potassium tartrate, sodium or potassium eitrate. This test has points of superiority over Fehling's test. In the first place the reagent can be made up in one solutoin instead of two, since the citrate does not tend to reduce the copper, even on long standing. Since sodium carbonate is not so strong an alkali as the hydroxide, there is less danger of a trace of sugar being destroyed. Benedict's test will detect a lower concentration of sugar than will Fehling's, and has replaced it in many laboratories.

Barfoed's Test.-This test also depends upon the reduction of a copper salt (copper acetate) by the sugar. It is performed, however, in acid solution. Barfoed's reagent contains copper acetate and acetic acid. The reducing action of the carbohydrates is very much less in acid than in alkaline solution. All of the reducing carbohydrates will reduce Barfoed's reagent if boiled a sufficient length of time, producing red cuprous oxide as in the Fehling test. The monosaccharides, however, reduce Barfoed's solution faster than do the disaccharides in equal concentration, so that if properly used, this test may serve to distinguish between monosaccharides and disaccharides. But a concentrated maltose solution will reduce Barfoed's reagent more rapidly than a weak glucose solution, so that this fact should be borne in mind or erroneous conclusions may result.

Almén-Nylander Test.-Nylander's reagent.contains bismuth subnitrate, sodium potassium tartrate and potassium hydroxide. The part played by each constituent is similar to that in Fehling's solution. The bismuth subnitrate is reduced to black, metallic bismuth. The equations follow:

$$
\begin{aligned}
& \mathrm{Bi}(\mathrm{OH})_{2} \mathrm{NO}_{3}+\mathrm{KOH} \rightarrow \mathrm{Bi}(\mathrm{OH})_{3}+\mathrm{KNO}_{3} \\
& 2 \mathrm{Bi}(\mathrm{OH})_{3}+\text { Sugar } \rightarrow \mathrm{Bi}_{2}+3 \mathrm{H}_{2} \mathrm{O}+(\text { Sugar }+30)
\end{aligned}
$$

Certain substances which interfere with Fehling's test, have no disturbing influence on the Nylander reaction (uric acid, 
creatinine) so that this test is useful occasionally when the Fehling test is of questionable value. A solution containing $0.08 \%$ glucose will give a positive Nylander test.

Haines' solution differs from Fehling's solution in containing glycerine in place of sodium potassium tartrate. Its delicacy is about equal to that of the Fehling test.

Reduction of Carbohydrates.-By the action of reducing agents carbohydrates may be converted into alcohols, or on further reduction they may give rise to compounds of the nature of fatty acids. Such transformations apparently occur in the cells of the body, for it is a well known fact that a carbohydrate diet is "fattening." Possibly the carbohydrate molecules are both split into fragments and reduced or dehydrated, and then recombined to form compounds with longer chains,-fatty acids. The exact mechanism of the process is still unknown. It is quite probable that carbohydrates may give up their oxygen to cells or microorganisms under conditions where vital activities are going on in the absence of atmospheric oxygen. This process is called anaërobic respiration.

Formation of Osazones.-Moriosaccharides and many of the disaccharides combine with phenylhydrazine to form osazones. These are yellow compounds which crystallize in needles. The crystals often group together with points at a common center, thus forming rosettes, or fans, or sheaves like grain sheaves. The different osazones have slightly differing crystal forms, but they are best recognized by their melting points; identifying an osazone serves to identify the sugar from which it was formed. Glucose and fructose form the same osazone, since the structure of these two sugars differs only around the carbon atoms to which the phenylhydrazine molecules become attached. This test thus will not distinguish between these two sugars. Saccharose, for a reason to be seen later, does not form an osazone, nor do the polysaccharides.

The reaction takes place in three stages as follows: 


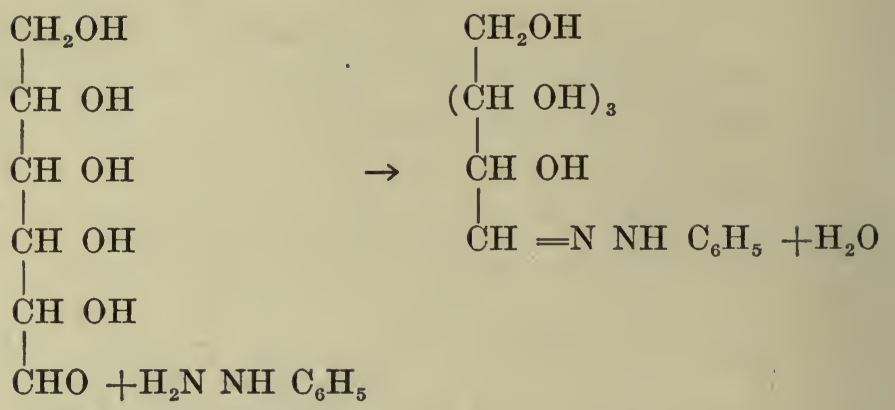

The product is a hydrazone. A second molecule of phenylhydrazine then removes two $\mathrm{H}$ atoms from the group next the end carbon atom, and is converted into aniline and ammonia.

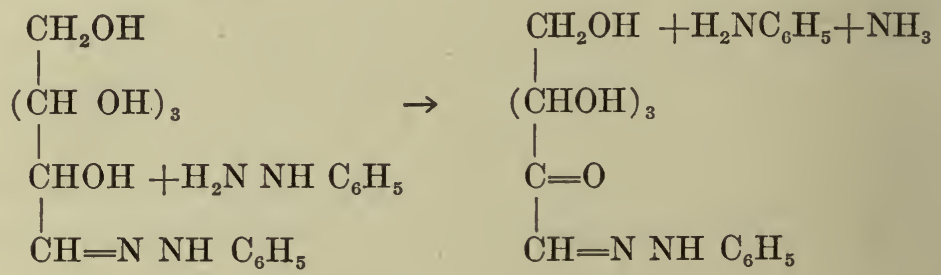

A third molecule of phenyl hydrazine now reacts, forming the osazone.
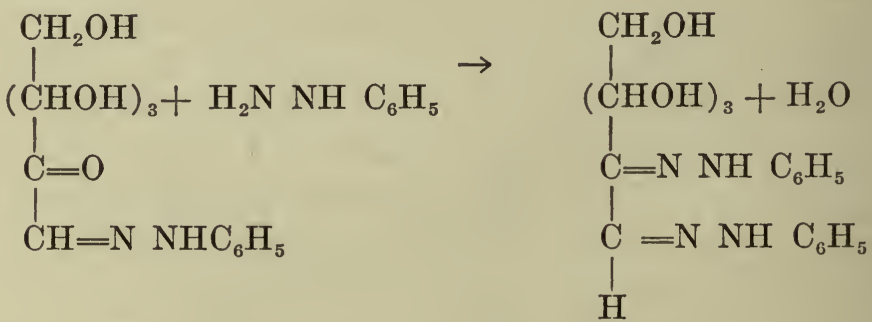

In place of phenyl hydrazine, its hydrochloride often is used, as this compound is more soluble and more stable than the free base.

The Molisch Test.-If to a solution containing a carbohydrate a few drops of $15 \%$ alcoholic $\propto$ naphthol are added, and 
concentrated sulphuric acid carefully poured down the side of the test tube so that it will form a layer at the bottom of the tube, a violet or reddish ring will form at the juncture of the two liquids. This test will detect not only free carbohydrate, but also carbohydrate in combination with other substances. The reaction is so delicate, however, that it will be given by very small traces of carbohydrate material, even fibers of filter paper (cellulose) so that as a general test it is more valuable in a negative than in a positve result. A negative Molisch reaction is good evidence that carbohydrates are absent, whereas a positive test may be due to the presence of filter paper or other casual impurities. As little as $0.2 \mathrm{mg}$. filter paper is said to give a strong reaction.

\section{Fermentation-Enzymes}

Under the influence of certain microorganisms the carbohydrates undergo a process known as fermentation, which consists in the breaking up of the carbohydrate molecule to form a variety of simpler compounds. The nature of the products depends upon the character of the particular organism responsible for the decomposition. Thus the carbohydrate may form carbon dioxide and alcohol, a process known as alcoholic fermentation. Or it may form lactic acid, butyric acid, or still other substances. In alcoholic fermentation we may represent the process as follows:

$$
\mathrm{C}_{6} \mathrm{H}_{12} \mathrm{O}_{6} \rightarrow 2 \mathrm{C}_{2} \mathrm{H}_{5} \mathrm{OH}+2 \mathrm{CO}_{2}
$$

but in reality there are intermediate steps the character of which is but imperfectly understood. The changes brought about in the sugar in these reactions are due to the fact that the microorganisms involved contain or secrete compounds which have the power of breaking down the sugar. We know a great many of these substances, and their activities are by no means limited to the breaking down of sugars. To them has been given the name of enzymes and different members of this class of substances have the power of bringing about the most widely vary- 
ing chemical reactions, both breaking down substances and building them up.

Enzymes have been the object of much study in recent years, but as yet very little is known of their chemical constitution. There is some evidence indicating that perhaps some of the members of the group may be, or may closely resemble proteins, whereas others appear to be carbohydrates. It is altogether probable that they will be found to vary much in their chemical nature, just as they vary in their chemical activities.

Although little is known of the chemical constitution of the enzymes, many important facts are known concerning their properties and the conditions governing their activities. One of the striking facts of enzyme action is that these substances do not appear combined with the final products which they produce. They are responsible for the breaking down or building up of many classes of compounds, but apparently they only change the speed of reactions which, if given sufficient time, would go on of themselves. Substances which show this behavior are spoken of as catalytic agents, and are said to act by catalysis. Enzymes have been defined as substances produced by living cells, which act by catalysis.

A striking fact in connection with the action of enzymes, is that under their influence, reactions go on in the body at a low temperature which, if duplicated in the laboratory without the aid of enzymes, require very high temperatures or the action of strong chemical reagents. This is a general property of catalytic agent, for we know that in the presence of spongy platinum, hydrogen and oxygen will combine at a temperature far below that required in the absence of a catalyst. The final point of equilibrium in the reaction is not altered,--the speed of the reactions is merely altered, and reactions which are capable of being catalyzed are considered to be going on even in the absence of the catalyst, though perhaps at an infinitely slow rate. Catalysts, including enzymes, do not appear combined with the final products of the reaction. They are temporarily united to the substance acted on,- the substrate, but 
are set free when the action is accomplished. They are thus made available for transforming a new portion of material, and a given weight of enzyme may transform many hundred times its weight of substrate. One might think that under these circumstances the reaction would go on indefinitely, but as a matter of practical experience this is not the case. Various factors contribute to this result. In the first place, there evidently is a gradual destruction of the enzyme. Then also, many reactions of biological importance are reversible, and an accumulation of end products will slow up the process. These products themselves may affect the speed of the reaction either way. If they tend to speed up the process, the effect is called autocatalysis. If they tend to inhibit the action, the effect is called negative autocatalysis. Then also, there may be a slight amount of permanent combination between the enzyme and the substrate, thus diminishing the amount of the enzyme. All of these factors described above tend to slow down the reaction except autocatalysis. Enzymes as a rule behave as do other catalysts, but they are produced only by living cells, are always colloidal which influences their behavior, and are more easily destroyed than are inorganic catalysts. The velocity of enzyme action is that of a unimolecular action (only one substance involved in the reaction).

There are some facts which do not quite harmonize with the classification of enzyme action as truly catalytic. A catalyst is supposed to have no effect upon the character of a reaction, or its final point of equilibrium. The destruction of glucose by enzyme action may yield, however, under the influence of different enzymes, widely differing products,-e. g., $\mathrm{CO}_{2}$ and alcohol, lactic acid, or butyric acid and hydrogen. Apparently the enzymes concerned not only catalyze, but also direct the course of the action.

It was formerly believed that the enzyme of yeast which breaks down sugar into alcohol and carbon dioxide acted only within the living cell, and that the vital activities of the cell were intimately connected with its action. Organisms of the 
type of yeast were called ferments. Another class of reactions was known to be independent of the cell by which the active principle was produced; substances responsible for such reactions were called unorganized ferments. Among them were the various digestive enzymes. In 1897 this fallacy was corrected by Buchner, who ground up yeast cells with sharp sand, thus tearing the cells and allowing the cell fluids to escape. The entire mixture of sand and broken cells was then pressed in a powerful press. A small quantity of liquid was obtained and was filtered through a porous porcelain filter which held back any fragments of cells. The clear liquid was found to decompose sugar in the same way as the original yeast cells had done. The activities of the enzyme were thus in no way dependent upon the vital processes going on in the cell. It is not to be supposed from this that the enzyme activities are of no value to the cell. Quite the contrary is the case, for undoubtedly the greater number of the chemical reactions which go on in the cell, and which to a large extent make up the sum total of what we call its vital activities, depend upon simple chemical reactions which are directed or controlled by enzymes. We still know certain types of enzymes which thus far have not been isolated from the cells in active form, but possibly this will be accomplished in the course of time.

Enzymes which normally act within the cell by which they are produced are called intracellular, or endoenzymes. Those which normally are secreted, and act outside the parent cell are called extracellular, or exoenzymes. The former are of great interest in pathological processes. The latter include the enzymes which carry out the processes of digestion in the alimentary tract.

Nomenclature and Classification.-The nomenclature of the enzymes is somewhat irregular, as several of the substances were named before any regular plan had been adopted. The ending "ase" is now employed to indicate that a substance is an enzyme, and the remainder of the word usually indicates either the substance upon which the enzyme acts, the nature of 
the reaction it brings about, or some other property of the compound. The substance upon which the enzyme acts is called the substrate. The classification of the enzymes is only provisional until a better one is possible. They are grouped according to the substances acted upon, or the character of the reaction induced, thus there are proteases (act on proteins), lipases (act on fats), amylases (act on starch and amylum), lactase (on lactose), maltase (on maltose), oxidases, reductases, etc. Of the older names we have pepsin, rennin, trypsin, zymase (found in yeast), etc.

The suffixes "lytic" or "clastic" are frequently used to indicate breaking down of the substrate. Many enzyme actions are hydrolytic in character, and the enzymes concerned are classed as sucroclastic, lipoclastic (or lipolytic), proteoclastic (or proteolytic) according to the type of substance acted on. Other types are desaminases, which remove the amino group from amino acids, carboxylases, which remove $\mathrm{CO}_{2}$ from the carboxyl group- $\mathrm{COOH}$, and coagulases, which coagulate proteins.

Specific Nature.-As may be inferred from the above statements, the enzymes act only upon particular substances or classes of substances, and in general an enzyme which acts upon one compound will not act upon any other. The enzymes are thus said to be specific in their action,- that is, each one acts only on a particular kind of material, or brings about one particular kind of chemical action. Emil Fischer, one of the most brilliant chemists of all times, has likened this characteristic to the fitting of a key with its lock. As a matter of fact, the enzymes are believed to fit onto the substances they act upon, forming temporary compounds which quickly break down again.

The degree of specificity is truly remarkable, for, of two compounds differing only in the slightest detail of stereochemical structure or arrangement of groups, an enzyme frequently will decompose one and leave the other untouched. This strengthens Fischer's key-lock idea. The enzyme is probably taken up, or adsorbed by the substrate and a slight difference in molecular structure may interfere with this process. 
Influence of Temperature.-One of the characteristics of the enzymes is their extreme sensitiveness to temperature. The temperature of the solution in which an enzyme is acting will be found to influence greatly the speed of the action. The temperature at which different enzymes will act best is known as their optimum temperature, and for the enzymes in the body this is in the neighborhood of $37^{\circ}-40^{\circ} \mathrm{C}$., in other words about body temperature. The enzymes in some of the cold-blooded animals, however, whose body temperature varies with that of their surroundings, act well at temperatures much lower than this, and it is obvious that enzymes in plant cells must work well at temperatures much below that of the body. If a solution containing an enzyme is heated to $60^{\circ}-80^{\circ} \mathrm{C}$., the enzyme is destroyed, and cooling the solution will not restore its activity. On the other hand, enzymes in general ean be exposed to temperatures near the freezing point. Their activity is retarded but will return if the solution is warmed.

In the existence of an optimum temperature for each enzyme, these substances differ from inorganic eatalysts, for which there usually is no optimum temperature, and which usually are not destroyed by heat, whereas all enzymes are destroyed in solution by heating to temperatures often much below $100^{\circ} \mathrm{C}$.

Effect of Chemical Reaction.-Enzymes are also very sensitive to chemical reaction. They are destroyed by strong acids or alkalies. The most favorable reaction differs with different enzymes, some acting best in weak acid, others in weak alkaline solution. Certain of the enzymes which act in weak acid solution are destroyed by making the solution even faintly alkaline, and the converse case is also true. Some, on the other hand, will stand considerable variation in this respect, acting in weak acid, in neutral or in weak alkaline solution.

Tenth normal acid and alkali are the limits beyond which enzyme action ceases. The optimum $\mathrm{pH}$ for an enzyme is much influenced by a variety of conditions. For example, certain proteolytic enzymes will digest one protein best at one $\mathrm{pH}$, and another protein at another $\mathrm{pH}$. Also the presence of salts or 
other substances may influence the optimum $\mathrm{pH}$ for a given action. Langfelt recently has shown that the optimum $\mathrm{pH}$ for liver amylase in the presence of chlorides is 6.8 , in the presence of phosphates, 6.2 and in the presence of adrenaline, 7.73.

Reversibility.-Some of the enzymes have the power of causing a chemical reaction to go either way, - that is, of decomposing a compound, or under proper conditions of building up the same compound from its decomposition products. Certain of the lipases are notable examples. The property is spoken of as reversibility, and these enzymes are said to be reversible in their action.

This phenomenon was demonstrated by Croft Hill, working with maltose and maltase, and he was able to show that the enzyme caused a resynthesis of disaccharide from the glucose produced. Kastle and Loevenhart on mixing butyric acid and ethyl alcohol with pancreatic extract were able to detect the odor of ethyl butyrate, indicating that the lipase of the panereas had caused a recombination of these substances to form the ester. The importance for the cell of the reversibility of enzyme action is apparent, for much of the work of the cell must be synthetic, either in building up or repairing its own structures, or in producing reserve materials for storage, such as glyeogen, fats, lipins, or other substances.

Active and Inactive Form.-In the form in which they are secreted by cells, some of the enzymes are inactive, and become capable of exerting their customary activity only after they have been acted on by some other substance. The enzymes are thus said to exist in inactive and active forms.

The inactive form is often called the zymogen form; the activating substance, the coenzyme or kinase. The coenzyme may be a salt, such as a chloride or phosphate, an acid, for example the gastric hydrochloric acid which activates pepsinogen. Coenzymes are usually unaffected by heat, so they obviously are not themselves enzymes. They often dialyze, and many enzymes are rendered inactive by dialyzing off the salts or other dialyzable substances in the solution. 
Action Retarded by Products.-The activity of an enzyme is often retarded by the products which it produces from the substrate. In life (in vivo) these products usually are removed, as in absorption of the digestion products from the intestine. In a laboratory experiment in a test tube or beaker, this may influence the extent of digestion by an enzyme to a considerable extent. This fact may be summed up by saying that enzyme action often is incomplete in vitro (in glass).

This topic already has been discussed under "autocatalysis" at an earlier point in the consideration of enzymes.

Progressive Action.- The enzymes are often said to be progressive in their action. This is only a special case of their specific nature. Many reactions depend upon more than one enzyme for completion, the reaction taking place in successive stages, each one of which is brought about by a different enzyme. Thus the breakdown of starch into glucose requires at least two and possibly more enzymes. The first breaks down the starch through various stages to maltose, the second breaks the maltose into glucose.

\section{Antienzymes-Defensive Enzymes}

If a rennin solution is injected into the blood of an animal, a substance appears in the blood which is capable of destroying the rennin. Such a substance is called an antienzyme or paralyzer. If various other substances, foreign to the body, are introduced into the blood, enzymes are produced capable of destroying them. Abderhalden has called these substances "defensive enzymes." This is referred to later in the discussion of the Abderhalden reaction for pregnancy. It truly is remarkable that the body tissues should be able to produce such substances for defense against compounds which they surely never can have been called upon to meet, either in the development of the individual or of the race. The secret to this problem may hold the explanation of the mode of enzyme production and enzyme action.

Summary.-In summary it may be said that enzymes are sub- 
stances of unknown chemical constitution, which act as catalytic agents affecting a large variety of chemical reactions. They are specific in their action, they are sensitive to changes in reaction and temperature, being destroyed if heated to $60^{\circ}-80^{\circ} \mathrm{C}$. Some are reversible in their action, they are often secreted in inactive form and become active only on coming in contact with some other substance, their action is often incomplete in vitro, and frequently is progressive in its character.

On the activities of enzymes depend a very large number of the processes by which the cells, and hence the tissues and the body as a whole carry on their various activities.

\section{Individual Groups of Carbohydrates.}

Pentoses.-The pentoses are found in both plants and animals, usually combined with other substances as in nucleoproteins or in the form of polysaccharides made up of many molecules of pentose. They are obtained by the hydrolysis of these compounds. A pentose, probably arabinose, has been found in the urine. This condition is known as pentosuria. Pentoses may be either aldoses or ketoses. They reduce Fehling's and other similar solutions, and give osazones with phenylhydrazine. They usually do not ferment, however. The pentoses are utilized by herbivorous animals but the extent to which they may be utilized by man seems to be more limited, although the subject is still a matter of some uncertainty. Pentoses may be distinguished from hexoses by their osazones, by their failure to ferment readily, and also by certain color reactions among which are the orcin and phloroglucin tests. If a pentose is heated with concentrated hydrochloric acid and a little orcin or phloroglucin, a distinct color change results. With orcin the color is first violet, then blue, red, and finally green, and a bluish green precipitate forms. With phloroglucin, the color is red. As other substances will give similar colors, it is necessary to confirm the result by observing the absorption spectrum of the colored substance after it has been dissolved out by shaking the liquid with amyl alcohol. The orcin test gives an absorption band between 
the $\mathrm{C}$ and $\mathrm{D}$ lines, the phloroglucin test, between the D and $\mathrm{E}$ lines. See discussion of absorption spectra below.

This procedure does not distinguish pentoses from glucuronic acid, however.

Arabinose is obtained by the hydrolysis of gum arabic, cherry gum, peach gum, etc., with dilute acid. It sometimes occurs in the urine. It has a sweet taste, and its solution is dextrorotatory $\left(1\right.$-arabinose has a specific rotation $\left.+104.5^{\circ}\right)$. Its melting point is $160^{\circ}$. Its osazone melts at $163^{\circ}-164^{\circ}$. Xylose is obtained by hydrolyzing wood, gum, straw, bran, etc. It often is called wood sugar. The pentose isolated from the nucleoprotein of the pancreas is said to be d-ribose. Its solution is levorotatory. The specific rotation of l-xylose is $+18.1^{\circ}$. Its phenylosazone meits at $155^{\circ}-158^{\circ} \mathrm{C}$.

\section{Absorption Spectra.}

White light is made up of a great many different colored lights as may be demonstrated by passing a beam of white light through a prism and observing the spectrum resulting from spreading out the different colored components of the original ray. Colored light may be either light of a single color or wave length, or it may be a mixture of many different colored lights, the sum of which, however, falls short of making a complete spectrum, or in which one color greatly predominates in intensity over the other colors present. If light passes through water, it still appears as white light, for the water has allowed the ray to pass through intact. If a ray of white light passes through a solution of hemoglobin, the red pigment of the blood, or of an amyl alcohol solution of the compound made by heating a pentose with orcin and hydrochloric acid, the ray no longer looks white, because the solution has absorbed or reflected a portion of the colored light, allowing only the red and perhaps some neighboring kinds of light to pass through. If the light is spread out in a spectrum, a portion will be missing. Many substances in solution have the property of absorbing particular kinds of light in this way, thus leaving a blank dark area in 
the spectrum if the emerging light is analyzed by spreading it out into its spectrum. The property is so constant that it may be made use of to identify compounds. The spectra resulting are called absorption spectra, and each absorption spectrum is characteristic of a particular substance. The lines or dark areas are charted with reference to the dark lines always observed in the spectrum of sunlight. These lines are called the Frauenhofer lines. The important ones are as follows:

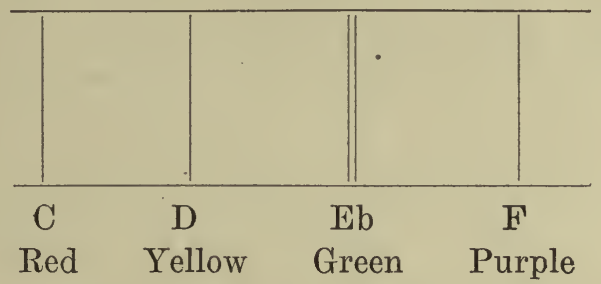

By looking through a solution with a spectroscope it thus is possible to identify many compounds of biological importance.

\section{Hexoses. $\mathbf{C}_{6} \mathbf{H}_{12} \mathbf{O}_{6}$}

Glucose. (Dextrose, grape sugar).-Glucose is found in both plants and animals. In the plant world it occurs in grapes and other sweet fruits, in seeds, roots, etc., and as a constituent of di- and polysaccharides and glucosides is much more widely distributed. In animals it is found in the blood and lymph, and occasionally in the urine. If the amount in the urine is more than a trace and it is found regularly, the condition is pathological and no time should be lost in consulting a physician. Glucose also is found in honey, an animal product. It may be obtained by boiling starch, glycogen, dextrins, etc., with dilute acid. Glucose erystallizes readily. It is soluble in water. The solution is less sweet than that of cane sugar. It is dextrorotatory, the specific rotation varying somewhat with the concentration. The figure usually reported is $+52.5^{\circ}$ for a solution in which equilibrium has been reached. It shows strong mutarotation. It is slightly soluble in warm alcohol and insoluble in 
ether. It gives all the reduction tests, ferments readily with yeast, forms caramel on warming with alkali, and with phenylhydrazine forms an osazone which melts at $205^{\circ}$. It is perhaps the most interesting of the sugars, for it is found in the blood, and serves as one of the most valuable fuels for the body cells. By the oxidation, or burning of glucose the cells praduce heat and do mechanical work.

Fructose. (Levulose, Fruit Sugar.)-Fructose is found in plants chiefly combined with glucose as cane sugar, or in the polysaccharide inulin from which it may be obtained by hydrolysis. It also occurs in honey. It is sometimes, though rarely, found in the urine in a condition known as levulosuria. The solubilities of fructose are similar to those of glucose. Its solution rotates the plane of polarized light to the left, the specific rotation being $-92^{\circ}$. It is called d-fructose because of its structural relationship to d-glucose, so that in this case the " $d$ " does not indicate that the compound is dextrorotatory. Solutions of fructose show the phenomenon of mutarotation. Fructose is a ketone sugar, and gives all the usual reduction tests for carbohydrates. It forms the same osazone as glucose, so that this test is of no value to distinguish the two sugars. Fructose also ferments with ordinary yeast. Fructose forms a calcium compound which is much less soluble than that of glucose and serves to separate the two sugars when they occur in a mixture.

Fructose may be distinguished from glucose by its levorotation, and also by the Seliwanoff reaction. On adding a few crystals of resorcinol, and concentrated hydrochloric acid to a levulose solution, and heating, a red color results. Glucose will give the test under certain circumstances, however, so that it must be carried out under definite conditions or it may lead to erroneous conclusions.

It also is possible to distinguish these sugars by means of methylphenylhydrazine. With this substance, fructose and other ketoses form osazones, whereas glucose and other aldoses form only hydrazones.

d-Galactose.-Galactose occurs in nature as a constituent of several gums, in the polysaccharide galactan in sea-weed, as a 
constituent of lactose or milk sugar and in certain substances in brain and nerve tissue. It is prepared from milk sugar or from various gums by hydrolysis. Galactose is somewhat less soluble in water than glucose. The solution is dextrorotatory, the specific rotation being $+81^{\circ}$. Galactose is an aldose and gives the usual reduction tests, and forms with phenylhydrazine an osazone which melts at $192^{\circ}-195^{\circ}$. Galactose ferments slowly but completely with ordinary yeast. If heated with nitric acid it forms mucic acid which is relatively insoluble and forms a fine white precipitate. This test serves to distinguish galactose from all sugars except lactose. It is of interest that the mammary gland constructs it out of the glucose of the blood, and unites it with glucose to form lactose or milk sugar.

Amino Sugars.-Closely allied to the monosaccharides are the amino sugars, which differ from the simple sugars only in having an amino group $\left(-\mathrm{NH}_{2}\right)$ instead of an $-\mathrm{OH}$ attached to the carbon atom next the aldehyde group. These compounds have been obtained by the hydrolysis of complicated substances occurring in the shells of lobsters and from the proteins mucin and mucoid which are widely distributed in the animal world. d-Glucosamine is an important member of this group. It is obtained by boiling the chitin of lobster shells with hydrochloric acid. It is readily soluble in water, the solution being alkaline. Its hydrochloric acid salt shows solubilities similar to those of the monosaccharides. The solution is dextrorotatory, having a specific rotation of from $+70^{\circ}$ to $+74^{\circ}$ according to concentration. It reduces the ordinary carbohydrate reagents and gives an osazone identical with that formed from glucose. Glucosamine does not ferment, however. d-Glucosamine is an interesting compound because in composition it stands midway between the carbohydrates and a group of substances called amino acids, which are the simple units of which the proteins are composed.

d-Glucuronic Acid.-This compound is obtained from glucose by oxidation, and is found in the body in combination with other substances. These compounds are called conjugated glucuronates. Glucuronic acid has the formula: 


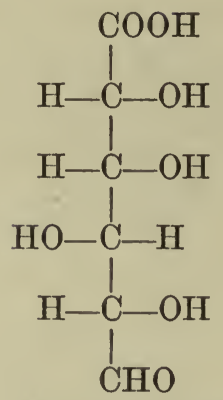

Glucuronic acid

It is interesting chiefly because it combires with various products formed by putrefaction in the intestine such as phenol, indol, skatol, etc. These substances are absorbed into the blood stream, and would exert a toxic influence upon the cells if it were not for the fact that the body promptly unites them to glucuronic acid, (or some other compounds) forming the conjugated glucuronates which are relatively harmless, and are excreted in the urine. This is one of nature's protective devices for shielding the cells against the influence of injurious substances. Solutions of glucuronic acid give the same reduction tests as glucose, are dextrorotatory, but do not ferment. The conjugated glucuronates are strongly levorotatory.

\section{Disaccharides.}

The disaccharides are formed by the union of two molecules of monosaccharide with loss of water.

$$
\mathrm{C}_{6} \mathrm{H}_{12} \mathrm{O}_{6}+\mathrm{C}_{6} \mathrm{H}_{12} \mathrm{O}_{6} \rightarrow \mathrm{C}_{12} \mathrm{H}_{22} \mathrm{O}_{11}+\mathrm{H}_{2} \mathrm{O} \text {. }
$$

By the action of dilute acids, enzymes, etc., they may be split into their constituent monosaccharides. The three disaccharides of importance are saccharose, lactose and maltose. These sugars are of great importance as food substances.

Saccharose. (Sucrose, Cane Sugar.) -Cane sugar is found in many plants, notably in the juice of the sugar cane, which contains about $20 \%$, and in carrots. It is found in many sweet fruits, such as the banana, strawberry, pineapple, etc., and in 
the sap of the sugar maple. It is a valuable food substance, and serves also as a condiment, by its sweetness making other foods more palatable. Cane sugar is prepared by treating the sap or juice containing it with milk of lime. This neutralizes any acids present, which otherwise would hydrolyze the sugar during evaporation. After being boiled to remove the protein, the calcium is removed by running in carbon dioxide, and the solution is decolorized either with animal charcoal or sulphur dioxide. After being boiled and filtered the liquid is evaporated in vacuo, and the cane sugar crystallizes out. The remaining liquid is known as molasses, and still contains considerable quantities of sugar which may be obtained by precipitation as calcium or strontium saccharate. From this compound the cane sugar may be set free with carbon dioxide.

Cane sugar is readily soluble in water, less so in alcohol, and insoluble in ether. The aqueous solution is very sweet, and is strongly dextrorotatory, the specific rotation being $+66.5^{\circ}$. The specific rotation of saccharose is practically independent of changes in concentration and temperature, so that the property is often made use of for its estimation. On hydrolysis it yields glucose and fructose.

On being heated to about $160^{\circ}$ cane sugar melts and if allowed to cool, forms a glassy mass which is known as barley sugar. At about $200^{\circ}$ it turns brown, forming caramel.

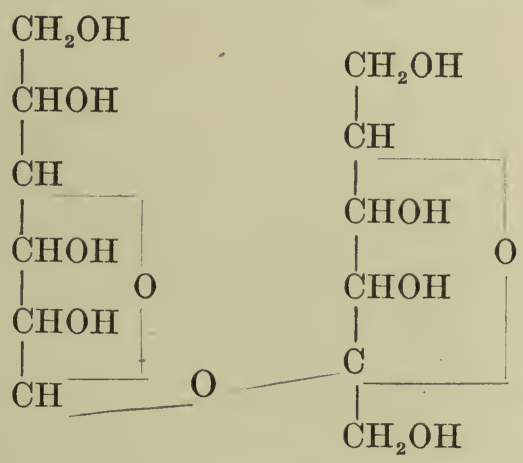

Saccharose 
Cane sugar does not reduce the usual carbohydrate reagents, such as Fehling's or Benedict's solutions and it does not form an osazone if treated with phenylhydrazine. This is due to its molecular structure. The aldehyde and ketone groups of its constituents are no longer free. On long boiling with these reagents, however, or after the action of an inverting enzyme on the cane sugar, the solution will give positive reactions with the above reagents since the sugar is split into its constituent parts. The hydrolysis or splitting of cane sugar is called inversion because the solution, originally dextrorotatory, is levorotatory after hydrolysis. This is due, of course, to the fact that the hydrolyzed solution contains equal quantities of glucose and fructose, the latter of which rotates more strongly to the left than does glucose to the right. The mixture is known as "invert sugar." A solution of cane sugar ferments readily with ordinary yeast, which contains an enzyme invertase which will invert the cane sugar. The resulting glucose and fructose are fermented by the zymase.

Lactose.-Lactose is found in the milk of all mammals, but does not occur in plants. Cow's milk contains about $4 \%$ lactose, human milk about $5 \%$ to $7 \%$. It may occur in the urine of women during pregnancy. Lactose is prepared from whey. On concentration, lactose crystallizes out, and may be purified

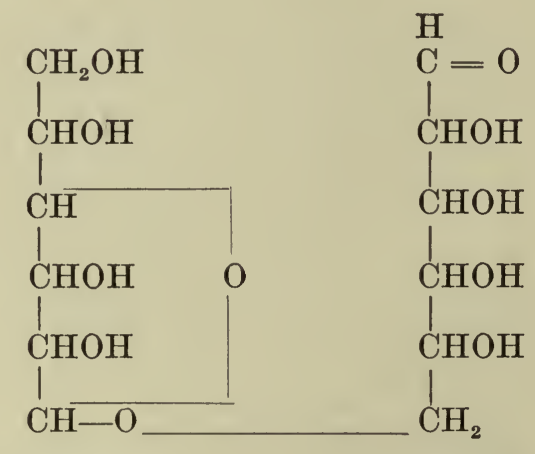

Galactose

Glucose

Lactose 
by recrystallization from water. The crystals are hard and gritty, and the solution is not so sweet as that of cane sugar. Lactose is composed of one molecule each of glucose and galactose. It is manufactured in the breast gland from the glucose of the blood. This is an interesting example of the conversion of one sugar into another in the body, since a portion of the glucose must be changed into galactose.

Lactose responds readily to the reduction tests for the monosaccharides. It thus possesses a free aldehyde group, and is represented by the accompanying formula. The solution of lactose is dextrorotatory. The specific rotation is $+52.5^{\circ}$. Lactose does not ferment with ordinary yeast. This property is serviceable in identifying it. It should be remembered that lactose will give the mucic acid test, since it contains galactose.

Maltose.-Maltose is formed in the hydrolysis of starch or glycogen by amylase. Since this enzyme is widely distributed in both plants and animals, maltose may be found wherever there is starch or glycogen.

Maltose is readily soluble in water. The solution is not so sweet as that of cane sugar. The solution is dextrorotatory, the specific rotation being $+136^{\circ}$. This value varies, however, with concentration and temperature. Maltose reduces Fehling's reagent, etc., and gives an osazone. Its structure is thus considered to be similar to that of lactose which has one free aldehyde group. On hydrolysis it yields two molecules of glucose. Maltose ferments readily with yeast. It may easily be distinguished from glucose by hydrolizing with dilute acid. After hydrolysis the reducing power of the solution will be found to have altered, since two molecules of glucose are now present for every molecule of maltose destroyed.

\section{Polysaccharides.}

Members of the polysaccharide group differ from one another considerably in their solubilities and other properties. They are found in both plants and animals in which they form reserve supplies of food material, and in plants and some of the lower 
animals, they are important constituents of the supporting framework or protective covering. The members of this group are made up of several molecules of monosaccharide united with loss of water to form the larger polysaccharide molecule: The commoner individual polysaccharides yield only one kind of monosaccharide when hydrolized, thus differing from the other material bases which, on hydrolysis yield varying kinds of simpler units. Since the number of monosaccharide molecules which make up a polysaccharide molecule is unknown, it is customary to express the formula with the indefinite coefficient $n$.

Starch $\left(\mathbf{C}_{6} \mathbf{H}_{10} \mathbf{O}_{5}\right)_{\mathrm{n}}$.- Starch is a plant product, and is found stored in leaves, seeds, fruits, tubers, etc. Grains contain as much as $50-70 \%$ of their dry weight; potatoes contain from $15-30 \%$ of their wet weight. Starch forms granules of more or less characteristic shapes which are serviceable in determining the source of the starch. Thus potato starch appears as eggshaped granules which often show concentric lines. Starch is prepared from potatoes or grain by grinding the material, filtering through sieves to remove the coarse debris and allowing the suspended starch particles to settle. It forms a white amorphous powder which does not dissolve in cold water. If boiled with water, the granules are broken open and the starch forms an opalescent solution. Starch is insoluble in alcohol and ether. A starch solution rotates the plane of polarized light to the right. It will not reduce Fehling's, or similar solutions, and will not ferment. The characteristic starch test is the formation of a blue color on the addition of a few drops of iodine solution. This color disappears on heating, but reappears if the solution is cooled. Alkali and alcohol also destroy the blue color. On boiling with dilute acids starch is broken down to glucose. The starch passes through various intermediate stages, the nature of which may be followed by the iodine test. If portions of the hydrolizing mixture are tested with iodine from time to time, the blue color soon gives place to red. This corresponds to the stage known as erythrodextrin (from the Greek word meaning "red"). On further hydrolysis, the iodine test 
gives no color. This is the achroodextrin stage (Greek word means "no color"). Beyond this stage maltose is formed, which then breaks up into glucose. The appearance of reducing sugars may be recognized, since the mixture will reduce Fehling's solution.

Starch is an enormously important food substance, and is widely used in the arts. In stiffening linen, the starch is broken down into dextrins by the heat of the iron. These dextrins give the fabric its stiffness and glossy appearance.

Dextrins.-Little need be said of the dextrins in addition to the fact that they are intermediate stages formed in the hydrolysis of starch to glucose. There probably are many members of the group, but so far, little is known of the different individual dextrins. Dextrins themselves probably do not reduce Fehling's solution, or only slightly, but commercial dextrin, which is prepared by the partial hydrolysis of starch, usually reduces Fehling's solution slightly, probably because the mixture contains maltose or glucose as the result of complete hydrolysis of some of the material. Dextrin solutions do not ferment, and give a red color (erythrodextrin) or no color (achroodextrin) with iodine according to the extent of the hydrolysis. Dextrins are readily soluble in water, but are precipitated by the addition of alcohol. The aqueous solution is dextrorotatory.

Inulin.-Inulin occurs in the sap of various plants and is found to the extent of $10-12 \%$ in the tubers of the dahlia. It is soluble in hot water, and gives a yellow or brownish color with iodine. It is interesting chiefly because on hydrolysis it yields levulose instead of glucose. It does not reduce Fehling's solution, and its solution is levorotatory.

Gums and Mucilages.-The gums and mucilages are widely distributed and on hydrolysis yield various pentoses and hexoses, and other substances. They vary greatly in their solubilities.

Cellulose-Cellulose is chiefly important in forming a large part of the structural framework of plants. The plant cell walls are made up of cellulose mixed with lignin and other sub- 
stances. Cellulose also is found in certain lower animals, the tunicates. Cellulose is insoluble in the ordinary solvents. It dissolves, however, in Schweizer's reagent, an ammoniacal solution of copper oxide, and in some other reagents. Cellulose derivatives are extensively used in the arts. Thus nitrocelluloses of varying composition are used in the manufacture of explosives, collodion, celluloid, artificial rubber, etc. From cellulose artificial silk and artificial gutta percha also are prepared.

There has been much discussion as to whether cellulose is of value as a food. Undoubtedly this is the case in herbivora. In man it probably is of little food value, but serves a useful purpose in giving bulk to the food and thus stimulating the muscular activity of the intestine. It has been stated that the cellulose of young and tender lettuce, asparagus, etc., may be utilized by the body to a considerable extent as food. There is no enzyme in the digestive juices capable of hydrolyzing cellulose, so that its disintegration must be due to the action of intestinal bacteria.

Glycogen.-Glycogen is found in the organs and tissues of animals, and also in some plants (yeast). It serves as a reserve fuel or food supply. The chief depots for glycogen deposit are the liver and the muscles. Glycogen is found in oysters, scallops and other molluses. Glycogen may be prepared from the liver of an animal which has just been killed. The liver is ground in a mortar with sand, and extracted with boiling water slightly acidified with acetic acid. If the extraction is not made at once after the death of the animal the glycogen supply will be greatly diminished or disappear altogether, as it is rapidly hydrolized to glucose by autolytic enzymes in the liver tissue. Feeding rabbits for a day or two on carrots before killing will insure a liberal supply of glycogen in the liver.

Glycogen is a white amorphous powder, which dissolves in cold water forming an opalescent solution. This solution gives a wine red or brown color with iodine. It does not reduce Fehling's solution, is not fermented by yeast, and is dextrorotatory. On boiling with dilute acids, glycogen is hydrolyzed to glucose. 
After hydrolysis the solution will of course, reduce Fehling's solution.

\section{Glucosides.}

Many compounds are obtained from plants and animals which yield on hydrolysis varying quantities of glucose or other simple sugars and in addition a wide variety of other compounds. To this group is given the name glucosides. Among these substances are many of the drugs used in medicine. The di- and polysaccharides themselves may be looked upon as glucosides, since in them glucose is combined with one or more sugar molecules. 


\section{FATS, PHOSPHATIDS AND ALLIED SUBSTANCES}

Distribution and Importance.-The fats are widely distributed in nature, in both plants and animals. In the former they are found in seeds such as cotton seed, the castor bean, etc., in fruits, such as olives, in nuts and also in the leaves and roots of some plants. In animals they are found in most tissues and fluids. The amounts in the tissues vary considerably. The active living protoplasm contains only about $1-10 \%$, whereas marrow, fatty tissue, etc., may contain considerably over $90 \%$. The fats are of importance as fuels for the body. They are laid away in large deposits which also serve the purpose of insulating the body by forming a blanket layer which aids in the conservation of heat. There is a layer of subcutaneous fat, and there are also large deposits around the abdominal viscera. Considerable quantities are found in the intramuscular connective tissue.

Composition and Structure.-The fats are made up of carbon, hydrogen and oxygen. The oxygen is present in much smaller per cent than in the carbohydrates. The constituent parts of the fats are the triatomic alcohol glycerine or occasionally some other alcohol, and organic acids, either of the fatty acid or a similar series. It is of interest that the acids making up the body fats have even numbers of earbon atoms in their molecules. The following list gives the names and formulas of some of the important acids:

$\begin{array}{lll}\text { Butyric } & \mathrm{CH}_{3} \mathrm{CH}_{2} \mathrm{CH}_{2} \mathrm{COOH} & \left(\mathrm{C}_{4} \mathrm{H}_{8} \mathrm{O}_{2}\right) \\ \text { Caproic } & \mathrm{CH}_{3} \mathrm{CH}_{2} \mathrm{CH}_{2} \mathrm{CH}_{2} \mathrm{CH}_{2} \mathrm{COOH} & \left(\mathrm{C}_{6} \mathrm{H}_{12} \mathrm{O}_{2}\right) \\ \text { Caprylic } & \mathrm{CH}_{3}\left(\mathrm{CH}_{2}\right)_{6} \mathrm{COOH} & \left(\mathrm{C}_{8} \mathrm{H}_{16} \mathrm{O}_{2}\right) \\ \text { Capric } & \mathrm{CH}_{3}\left(\mathrm{CH}_{2}\right)_{8} \mathrm{COH} & \left(\mathrm{C}_{10} \mathrm{H}_{20} \mathrm{O}_{2}\right) \\ \text { Palmitic } & \mathrm{CH}_{3}\left(\mathrm{CH}_{2}\right)_{14} \mathrm{COOH} & \left(\mathrm{C}_{16} \mathrm{H}_{32} \mathrm{O}_{2}\right) \\ \text { Stearic } & \mathrm{CH}_{3}\left(\mathrm{CH}_{2}\right)_{16} \mathrm{COOH} & \left(\mathrm{C}_{18} \mathrm{H}_{36} \mathrm{O}_{2}\right)\end{array}$


The acids listed above are all saturated compounds, that is they contain no double bonds. An acid found in a large number of fats is olcic acid, which has the same number of carbon atoms as stearic acid, but two less hydrogen atoms. It is thus $\mathrm{C}_{18} \mathrm{H}_{34} \mathrm{O}_{2}$ and its formula is

\section{$\mathrm{CH}_{3}\left(\mathrm{CH}_{2}\right)_{7} \mathrm{CH}=\mathrm{CH}\left(\mathrm{CH}_{2}\right)_{7} \mathrm{COOH}$.}

It is an unsaturated acid, and contains a double bond. Fats containing this acid have a lower melting point than those containing the corresponding saturated compound. Some allied compounds contain other alcohols in place of glycerine. Thus cetyl alcohol $\mathrm{C}_{16} \mathrm{H}_{33} \mathrm{OH}$ is found in spermaceti in the head of the sperm whale, and myricil alcohol $\mathrm{C}_{30} \mathrm{H}_{61} \mathrm{OH}$ in beeswax, etc. Esters of these alcohols usually are called waxes. The following formula illustrates the structure of a fat.

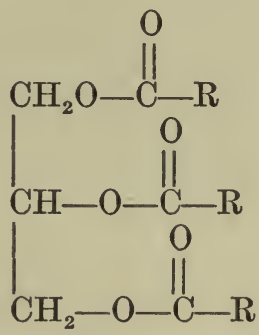

$R$ is the rest of an acid molecule. If the fat were tristearin, $R$ would represent a chain of $16 \mathrm{CH}_{2}$ groups with a $\mathrm{CH}_{3}$ group at the far end. Fats are thus tri-atomic esters of glycerine and an organic acid. The three R's may be all the same fatty acid, or they may be different. There is thus the possibility of having a large number of different fats, differing in the kind of fatty acid present. This possibility is realized in nature, and a large number of different fats are known. The naturally occurring fats are rarely made up of a single kind of fat, but usually are mixtures of various kinds such as tripalmitin, tristearin, and triolein, as the fats from these respective acids are called. Oleic acid has a very low melting point, and triolein also melts at a low temperature. The presence of much triolein in a fat lowers 
its melting point, often to such an extent that the fat is liquid at ordinary room temperature. Such fats are called oils. Other unsaturated acids are found in some fats, or "oils," and they exert a similar influence.

Among these unsaturated acids are linolic $\left(\mathrm{C}_{18} \mathrm{H}_{32} \mathrm{O}_{2}\right)$ and linolenic $\left(\mathrm{C}_{18} \mathrm{H}_{30} \mathrm{O}_{2}\right)$. Oils which contain considerable amounts of the esters of these acids undergo oxidation easily and are converted into a hard resinous material when exposed to air and light. They are called drying oils, and are used in paints and varnishes. Linseed oil is an example of this group. Others, such as cottonseed oil only thicken on exposure to air and light; they are called semidrying oils. Olive oil does not harden under similar circumstances and is classed as a nondrying oil.

General Properties.-The solid fats are white or light yellow substances, which if pure are odorless and tasteless. The oils often are yellow and frequently have a decided taste and odor. They are insoluble in water, somewhat soluble in cold alcohol but much more so in hot alcohol, and soluble in ether, chloroform, benzol, etc. From solutions, the fats often may be obtained in crystalline form as long needles. The specific gravities of all the fats and oils are less than that of water, hence they float at the surface. They reduce the surface tension of water. The naturally occurring fats and oils do not have sharp melting points, since they are mixtures of different kinds of fats. Even pure fats often show much indefiniteness in melting point. Some melt, resolidify at a slightly higher temperature, and if further warmed, melt again. This is supposed to be due to the fact that an internal rearrangement in the molecule is brought about by heating.

Emulsification.--If neutral oil and water are shaken together vigorously, and the mixture allowed to stand, it will quickly separate into two layers, oil and water. If a small amount of soap solution is added to this mixture and the shaking repeated, the liquid becomes milky in appearance, and even after prolonged standing will fail to separate into two layers. 
The fat is said to be emulsified. On examining such a mixture under the microscope, it will be seen to be filled with minute globules of oil suspended in the water. If the proportions are reversed, that is, if there is much oil and little water, the water will be suspended in the oil. Soap is by no means the only substance which will favor the formation of an emulsion. Albumin, gums such as gum arabic, and a variety of other compounds will bring about a similar result. Lymph will emulsify a fat, and if a drop of lymph and a drop of oil are brought in contact on a microscope slide, the oil may be seen to break up into minute droplets and enter the lymph drop.

Physiologically the formation of emulsions is of great importance. In digestion in the stomach only emulsified fats are attacked to any extent. In the intestine, fats of the food are emulsified by the pancreatic juice, a process which is extremely important for their proper digestion and absorption. The mechanism of emulsion formation has been the subject of much study. Probably different emulsifying agents act in different ways, or a single substance may act in more than one way. It is believed that the soap, albumin, etc., collects around the tiny fat droplets and serves to insulate them and thus lessen the tendency to run together. The lowering of the surface tension is also a factor in the production of emulsions, and possibly also electrical forces tending to repel the similarly charged particles of fat. Milk is an example of a fairly permanent emulsion. The fat droplets are suspended in a liquid which contains protein. On standing, a considerable portion of the milk fat finally will float to the surface. When removed from the skimmed milk beneath, this is known as cream. On churning, the emulsified fat runs together and butter is formed.

Saponification.-If a fat is boiled with an alkali, or an acid, it is split into fatty acids and glycerine. This process is known as saponification. If an alkali is used, the fatty acids react with the alkali to form salts. These salts of the higher fatty acids have a slippery feeling, and their solutions foam on being 
shaken. They are called soaps. The accompanying equation illustrates the process:<smiles>CCCC(=O)OCC(CCC)C(=O)OCC</smiles><smiles>CCCC(O)C(CO)C(=O)O[Na]</smiles>

The process is best carried out in alcoholic solution. Sodium soaps are known as hard soaps; potassium soaps which are buttery in consistency are known as soft soaps. Calcium soaps are very hard and insoluble. Soap is a useful eleansing agent. Soiled articles,-clothing, the hands, etc., usually are covered with a layer of fatty material which entangles and holds partieles of insoluble inorganic dirt. Soap emulsifies the fat and the remaining material is carried away by the water or by the lather, which takes up the particles of dirt mechanically.

Since calcium soaps are very insoluble, hard water is not good for washing purposes, as the calcium precipitates the soap added, and thus interferes with its cleansing activities.

Rancid Fats.-Many natural fats, upon standing, acquire a disagreeable taste and odor. This is due to the splitting of some of the neutral fat into glycerine and fatty acids. The lower fatty acids, such as those found in butter have a very disagreeable taste and odor, hence the character of "rancid" butter, etc.

Detection and Identification.-Acrolein Test.-Fats are easily detected by their physical properties, such as solubility, appearance, greasiness, etc. A test given by all common fats is known as the acrolein test. If a fat is heated to $300^{\circ}$ it is decomposed. The glycerine portion of the molecule loses water and forms the unsaturated compound acrolein. The test is obtained more readily if the fat is heated with a dehydrating 
agent such as potassium acid sulphate, boric acid or phosphorus pentachloride. Acrolein is easily recognized by its extremely sharp and irritating odor. Since only substances containing glycerine give the test, it may be used to distinguish between fats and fatty acids or soaps.

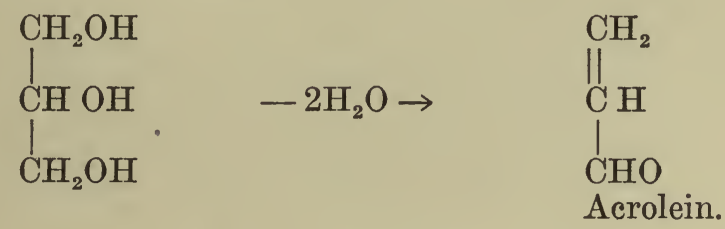

Melting Point.--The melting points of the natural fats are not sharp, since natural fats usually are mixtures. They often melt, solidify on further heating, and melt again at a higher temperature. Those fats whose melting points are below ordinary room temperature are called oils. The melting points of fats in animal tissues are generally below the usual temperature of those tissues, so that the body fats are in a fluid state. The fats of cold blooded animals melt at lower temperatures than those of warm blooded animals.

Saponification Equivalent.-The saponification equivalent is the number of milligrams of potassium hydrate necessary to neutralize the fatty acids produced by the saponification of one gram of fat. The smaller the molecular weight of the acids in the fat, the larger will be the number of molecules in a gram, and the higher the saponification number. Fats made up of fatty acids such as palmitic, stearic and oleic acid such as oleomargarine have a saponification number around 195. Butter, which contains fatty acids of low molecular weight has a saponification number around 227. These two substances may be distinguished easily in this way.

Volatile Fatty Acids.-Reichert-Meissl Number.-This method is used to give evidence of the amount of lower fatty acids in a fat. The fat is hydrolized with alkali, acidified with sulphuric acid and distilled. The fatty acids of low molecular weight distil over and may be titrated. Those of higher molec- 
ular weight are not volatile and remain behind. The number of cubic centimeters of $\mathrm{N} / 10$ alkali required to neutralize the volatile fatty acids from 5 grams of fat is called the ReichertMeissl number. For butter fat the number is 25-30.

Iodine Number.--The acids which contain double bonds, such as oleic acid will take up iodine or bromine, adding on two atoms of the halogen for each double bond. By this process it is possible to determine how much unsaturated acid is present in a fat. The weight of iodine in centigrams taken up by a gram of oil is known as the iodine number. Hydrogen and oxygen are taken up in an analogous manner, and this fact is also used to identify fats.

Acetyl Eqivalent.-Some fats contain oxyacids,-that is acids containing hydroxyl groups. These groups may be replaced by acetyl groups, which in turn may be split off and the resulting acetic acid titrated. The number of milligrams of potassium hydrate required to neutralize the acetic acid obtained from 1 gram of fat in which the hydroxyl groups have been replaced by acetyl groups is known as the acetyl equivalent.

The various factors described above have been determined for the important natural fats, and variations in one or more of these characteristic constants are of great service in determining whether a given fat or oil is pure, or has been adulterated.

Important Fats.-Tristearin, tripalmitin and triolein are the three fats occurring most frequently in natural fats.

Tristearin, or stearin, as it is often called, melts first at about $55^{\circ}$, resolidifies and melts again at about $71^{\circ}$. It is a hard, flaky material, and the least soluble of the three. It is obtained from tallow. Mixed with a little paraffin to make it less brittle it is moulded into candles. Free stearic acid is found in old pus, in gangrenous or tuberculous masses, etc., where decomposition of fat has taken place. It is found as its alkali soap in blood, bile, etc., and as its calcium soap in the feces.

Tripalmitin, or palmitin, is found in all animal and most 
vegetable fats, notably in palm oil, whence it derives its name. It predominates in human fat. It melts first at about $50^{\circ}$, resolidifies, and melts again at about $66^{\circ}$.

Triolein, or olein, is found in animal, and to a greater extent in vegetable fats and oils. It melts at $-6^{\circ} \mathrm{C}$, and is thus a liquid (an oil) at room temperatúre. The presence of olein lowers the melting point of natural fats. If exposed to the air olein quickly becomes rancid. Oleic acid, by reason of its double bond will take up iodine as described above.

Butter.-Butter, the fat obtained from cream, contains about $85 \%$ fats. About $7 \%$ of this is made of lower fatty acids. Of the remainder $60-70 \%$ is palmitin, and $30-40 \%$ olein. Butter contains very little stearin.

Oleomargarine is made usually from beef fat. The fat is melted, cooled, the oily portion pressed out and this mixed with various substances such as peanut oil, lard, etc., and finally churned up with milk to give it a butter flavor. If oleomargarine is made from clean, wholesome materials it is a perfectly satisfactory food substance. It often lacks certain substances found in butter, however, which are called vitamines, which recently have been shown to be very valuable materials to the body. Cod liver oil also contains these substances. Perhaps this fact justifies the popular impression of its beneficial nature.

Lanolin, or wool fat, which is an ester of higher fatty acids chiefly with the alcohol cholesterol in place of glycerine, is useful in medicine. It forms extremely fine emulsions and thus serves as a valuable basis for the formation of salves and ointments. Substances containing alcohols other than glycerine, such as cholesterol are often classed as waxes.

\section{Phosphatids, Cerebrosides, and Sterols}

A large number of substances have been isolated from animal and plant tissues which have certain similarities to the fats, such as solubilities, general appearance, etc. Some of these compounds are also related chemically to the fats. Substances of this nature are found in all cells, both 
animal and vegetable, and particularly in the brain and nerve tissues. Little is known of the biological function of these compounds, although as our information increases, they appear to be increasingly important. The composition of some of them is known, whereas in the case of others, we have no assurance that the substances reported are single compounds and not mixtures of closely related compounds.

The term lipoid has been used to designate these "fat-like" bodies, but the term has been used in so many different connections that MacLean suggests it be abandoned. He proposes the name "lipins" for the two groups, phosphatids and cerebrosides. These substances, which may be obtained from various tissues by extraction with ether, yield, among other things: fatty acids on hydrolysis. The phosphatids contain both nitrogen and phosphorus, and on hydrolysis yield phosphoric acid, glycerine, fatty acids, and a nitrogen base such as choline or amino-ethyl alcohol. They are classified on the basis of the relative amounts of nitrogen and phosphorus which they contain. Lecithin, kephalin and sphingomyelin are the best known members of the group.

The cerebrosides, obtained chiefly from the brain, contain nitrogen, but no phosphorus. On hydrolysis they yield, among other things the carbohydrate galactose or one of its derivatives. The only two substances definitely known to be individuals in this group, and not mixtures, are phrenosin and kerasin. Little is known of the functions of the cerebrosides, but their presence in the brain is sufficient to make them interesting.

Lecithin.-Lecithin, classed as a phosphatid, is one of the interesting compounds of this type. It is found in all cells, in greatest amount in egg yolk which contains about $10 \%$. Lecithin is soluble in absolute alcohol, and in ether, but may be precipitated from the latter solution by adding acetone. On hydrolysis lecithin yields higher fatty acids, glycerine, phosphoric acid and an organic base choline. It is believed to have the following formula, where $R$ indicates a fatty acid residue. 
FATS, PHOSPHATIDS, ETC.

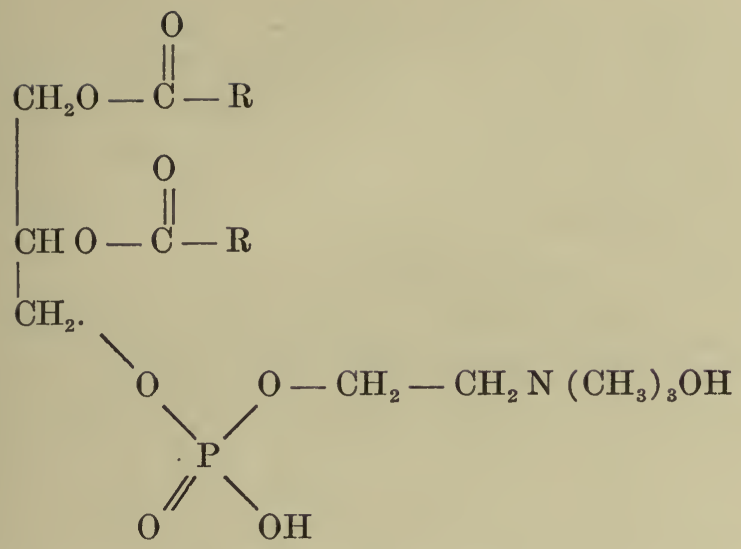

The functions of lecithin in the body are not perfectly understood. Recent studies have made it seem probable that much of the "fat" of the tissues, and the blood is in reality lecithin. It appears that the body easily synthesizes lecithin, so that it is not a necessary constituent of the diet. In fact the lecithin of the food probably is hydrolyzed by the lipase in the intestine into its constituent parts. Undoubtedly the lecithin in the cells aids them in holding water. Various other functions have been suggested, but in most cases the evidence is not conclusive.

Cholesterol.-Cholesterol is a substance which resembles the fats in some of its physical properties, but has little relation to them chemically. It is widely distributed in nature, and is found in large amounts in the brain and nerve tissue. It occurs also in small amounts in the blood, and in the bile, from which it is occasionally deposited in gall stones, whence it is most easily obtained. Cholesterol is insoluble in water, acids, or alkalies. It is readily soluble in hot alcohol, in ether, chloroform, benzol, etc. Cholesterol crystallizes from hot alcohol or other solvents, forming large colorless plates. It gives many color reactions. If a chloroform solution of cholesterol is carefully treated with concentrated sulphuric acid so as to form a layer of acid at the bottom of the test tube, the chloroform solution becomes a brilliant red, and the acid layer dark red with a 
green flucrescence. This test is known as Salkowski's test. The Lieberman-Burchard test is one of the best of the cholesterol tests. To 2 c.c. of a chloroform solution of cholesterol, 10 drops of acetic anhydride and 2 drops of concentrated sulphuric acid are added. A violet color appears which quickly turns to a blue green.

Cholesterol is an important substance physiologically. Apparently it protects the red blood corpuscles from certain harmful substances. It also seems to exert a restraining influence on some of the cell enzymes, and thus may act as a regulator of some of the cell activities. Cholesterol and certain of its allied substances are important in giving the cell its property of retaining the water which it contains. The presence of cholesterol in the brain in such large quantities points to the suggestion that it may have some important rôle in the functioning or properties of this most important organ. In short, there are doubtless numerous other rôles played by cholesterol in the body, of which we know very little as yet.

Cholesterol contains neither nitrogen nor phosphorus, and is classed as a sterol. It probably belongs to the group of terpenes. 


\section{CHAPTER V}

\section{PROTEINS}

Introductory.-The group of the proteins is of great importance in nature, both in plants and in animals. Members of this group are found in every living cell, and are necessary for the life of the organism. An animal may get on very well for a long time on a diet containing no carbohydrate or fat, but if fed on a diet containing no protein, the animal will die. Plants build up their own proteins from nitrogen compounds of the soil or air, but animals are dependent upon plants or other animals for the materials out of which they construct their proteins. Plant structures contain large amounts of carbohydrates along with protein and other materials, but the tissues of animals are made up largely of proteins. Thus muscle. nerve, ligament, skin, bone, blood, lymph, hair, nails, feathers, eggs, etc., all contain much protein. Often it is their chief solid constituent. In plants we find stores of protein laid away in seeds, such as the grains, and in many other places.

Elementary Composition.-The members of the group of proteins differ very widely among themselves in many properties, but they are all alike in certain respects, as for example in their elementary composition, for all proteins contain carbon, hydrogen, oxygen and nitrogen. Some contain also sulphur and some phosphorus. In addition to these elements we find sometimes others, such as iron, iodine, copper, manganese, etc. The proteins show individual variations in composition, but an average percentage is as follows:

$\begin{array}{lrlr}\mathrm{C} & 50 \% & \mathrm{~N} & 16 \% \\ \mathrm{H} & 7 \% & \mathrm{~S} & 0.3 \% \\ \mathrm{O} & 22 \% & \mathrm{P} & 0.4 \%\end{array}$


The earbon forms the backbone of the protein as long chains to which the other elements are attached. Oxygen is present in a proportion smaller than in the carbohydrates, but greater than in the fats. Nitrogen is present in various groupings, chiefly as imid-NH- groups which form the links holding the various parts of the protein together; as amino groups $-\mathrm{NH}_{2}$; and as $-\mathrm{CONH}_{2}$. These last two forms represent only a small part of the total nitrogen. Sulphur is present for the most part in unoxidized form - $\mathrm{S}$ - in one or two of the constituents of the protein molecule, but may be present also in oxidized form. Phosphorus is present perhaps in different forms, chiefly in oxidized form as phosphate.

Classification.-A knowledge of the percentage composition such as that given above affords us no clue to the structure of the protein molecule, which is very complex. Our knowledge of the actual structure of the proteins is so limited that a classification of the group has been worked out chiefly along other lines, making use of solubilities, source, etc., to distinguish groups, in connection with knowledge of chemical components so far as such information was available. The classification adopted by the American Society of Biological Chemists is as follows :
I. Simple Proteins.
II. Conjugated Pro- TEINS.
1. Albumins
2. Globulins
3. Glutelins
4. Prolamines
5. Albuminoids
6. Histones
7. Protamines
1. Glycoproteins
2. Phosphoproteins
3. Hemoglobins
4. Nucleoproteins
5. Lecithoproteins
III. Derived Proteins. A. Primary Protein De- rivatives.
1. Proteans
2. Metaproteins
3. Coagulated Pro- teins
B. Secondary Protein Derivatives
1. Proteoses
2. Peptones
3. Peptids

This classification is by no means final or ideal. Compounds are known which do not fall well into any group, whereas others seem to be intermediate between two groups. This classification serves very well, however, to bring comparative order 
out of chaos until more is known of the structural differences which differentiate the various members of the group. Another classification, in use by English biochemists, differs only slightly from that given above. Hemoglobins are called chromoproteins; albuminoids, scleroproteins; and the third division is subdivided somewhat differently in the English classification.

\section{Preparation of Proteins from Ilaterials in Which They Oc-} cur.-Most proteins occur naturally mixed with a large number of other materials. Only a few are found in a fairly pure state in nature. For purposes of study it is desirable to get them as free as possible from other compounds. Various methods are useful, according to the properties of the protein to be isolated and those of the other substances present in the mixture or tissue. But even at best the purification of a protein is a difficult task and when finished it is often uncertain whether or not the substance is contaminated by some other protein of very similar composition and properties or by other compounds. Plant proteins often may be dissolved out with $10 \%$ sodium chloride or with alcohol and separated from one another by dialyzing the salt, or by fractional precipitation. In preparing proteins from animal tissues, the process is made somewhat more difficult by the fact that such tissues contain larger amounts of autolytic enzymes. These have the property of breaking down the cell constituents or altering them if the tissues are allowed to stand for some time before the proteins are extracted. Also, it is difficult to free the proteins from the fatty or phosphatid constituents of the cell without altering the nature of the protein itself. Various solvents may be used, such as salt solutions, dilute acids or alkalies. A method which is very satisfactory consists in freezing the tissue quickly, reducing it to a fine powder and drying while still frozen. This material then may be extracted with petroleum ether to remove fats, phosphatids, cholesterol, etc. The residue is extracted with $10 \%$ sodium chloride solution, and the various proteins separated by fractional precipitation.

Some of the proteins may be purified by re-crystallization. 
Molecular Weight.-The molecular weight of the proteins is very high. Estimation of the molecular weight has been attended by great difficulties, because of the practical impossibility of obtaining pure proteins, and because of the unstable nature of the proteins themselves. The molecular weight of these compounds is so great that they cause only a very slight lowering of the freezing point, so that this method of determining molecular weight has given results of questionable reliability. The boiling point method cannot be used, as proteins coagulate on heating. Various other methods have been devised, however, and as fairly uniform results are obtainable by different methods of analysis, we may draw fairly accurate conclusions as to the molecular weights of members of the group. One of the methods employed consists in estimating the sulphur content of the protein. If the substance contains $0.5 \%$ sulphur then sulphur makes up $1 / 200$ of the protein, and the molecular weight will be 200 times that of the sulphur present. If there are two sulphur atoms in the protein, they have a molecular weight of $64(2 \times 32)$. The molecular weight of the protein will thus be $200 \times 64=12,800$. Calculated on this basis most of the proteins will have a molecular weight of from 14,000-16,000. Calculating the molecular weight in other ways, such as from the amount of oxygen some of the proteins will take up, gives practically identical figures. More direct methods (measurements of osmotic pressure, etc.) confirm these figures, so that we are justified in assuming the molecular weight of most proteins to be roughly in the neighborhood of 15,000 , although some recent evidence makes it seem that perhaps these figures, after all, are far too high. Comparing this figure with the molecular weights of some familiar compounds such as hydrochloric acid $(36+)$, sodium hydrate $(40)$ and sodium chloride $(58+)$, we get an idea of the relative hugeness of the protein molecule.

Hydrolysis.-Any consideration of the molecular structure of compounds having such enormous molecules would seem to present almost insurmountable obstacles. As the result of the work chiefly of Kossel, Emil Fischer, Emil Abderhalden, and 
Osborne, however, much light has been thrown on the subject. It has been found that proteins if boiled with concentrated acids, with alkalies, or if acted on by certain enzymes will break down ultimately into fairly simple chemical substances. These are the $\propto$ amino acids. On hydrolysis, all proteins are broken down into a mixture of these compounds, of which about twenty have been obtained from proteins. All proteins, whatever their nature, yield these same compounds. All the amino acids are not obtained from every protein, but in general a protein yields most of them. Many proteins lack one, two, three, or perhaps more, and a few proteins are made up of relatively few different amino acids. The analytical methods in use do not give products to the amount of $100 \%$ of the original protein, for there are losses at various stages in the process. Usually only about $2 / 3$ of the original substance is accounted for. From some proteins $85-90 \%$ has been recovered, and in the case of salmine $110 \%$, this apparently impossible result being accounted for by the taking up of water when the amino acid complexes are split up. It is easy to understand the causes for the difference in behavior of different proteins. Although they all are made up of practically the same amino acids, these are present in different proteins in widely varying proportions, and also undoubtedly are arranged or put together differently.

The following is a list of the amino acids which thus far have been obtained by the hydrolysis of proteins. It is quite possible that as time goes on others will be added.

\section{Amino Acids Obtained by Hydrolyzing Protein}

A. Monoamino-monocarboxylic acids.

1. Glycocoll

2. Alanine

3. $\propto$ Amino Butyric

4. Valine
$\mathrm{NH}_{2} \mathrm{CH}_{2} \mathrm{COOH}$

$\mathrm{CH}_{3} \mathrm{CHNH}_{2} \mathrm{COOH}$

$\mathrm{CH}_{3} \mathrm{CH}_{2} \mathrm{CHNH}_{2} \mathrm{COOH}$

$\mathrm{CH}_{3}$

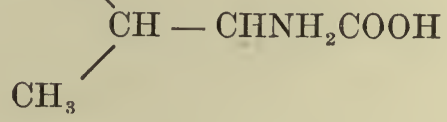


5. Caprine

$$
\mathrm{CH}_{3} \mathrm{CH}_{2} \mathrm{CH}_{2} \mathrm{CH}_{2} \mathrm{CHNH}_{2} \mathrm{COOH}
$$

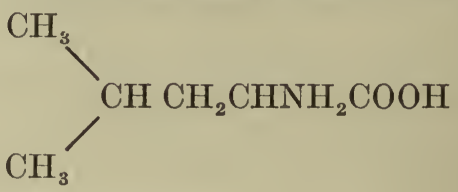

6. Leucine

7. Isoleucine

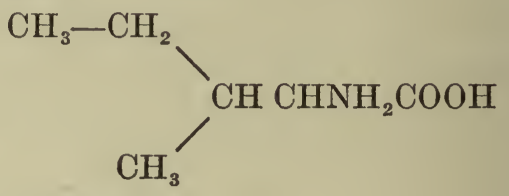

8. Serine

$$
\mathrm{CH}_{2} \mathrm{OH}
$$<smiles>CC(N)N</smiles><smiles>CCC(=O)O</smiles>

\section{$\mathrm{CH}_{2} \mathrm{SH}$}

9. Cystein

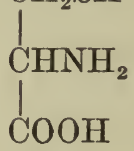

10. Cystin

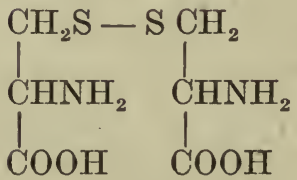

B. Monoamino dicarboxylic acids.

11.

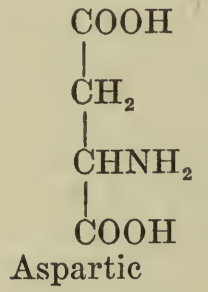

12. $\mathrm{COOH}$

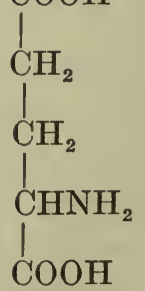

Glutamic 
C. Diamino-monocarboxylic acids

13. Lysine

$\mathrm{NH}_{2} \quad \mathrm{NH}_{2}$

13. Lysine

14. Arginine

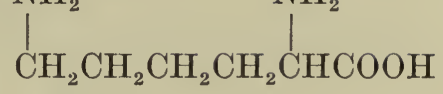

D. Cyclic compounds.

15. Phenylalanine

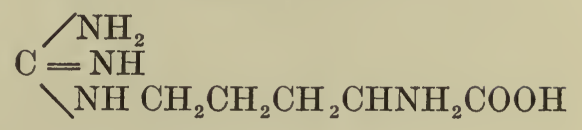

16. Tyrosine

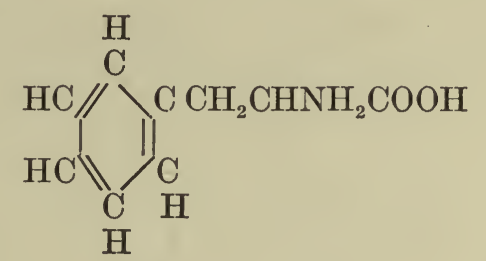

17. Tryptophane<smiles>NC(CNC(=O)O)Cc1ccccc1</smiles>

18. Histidine
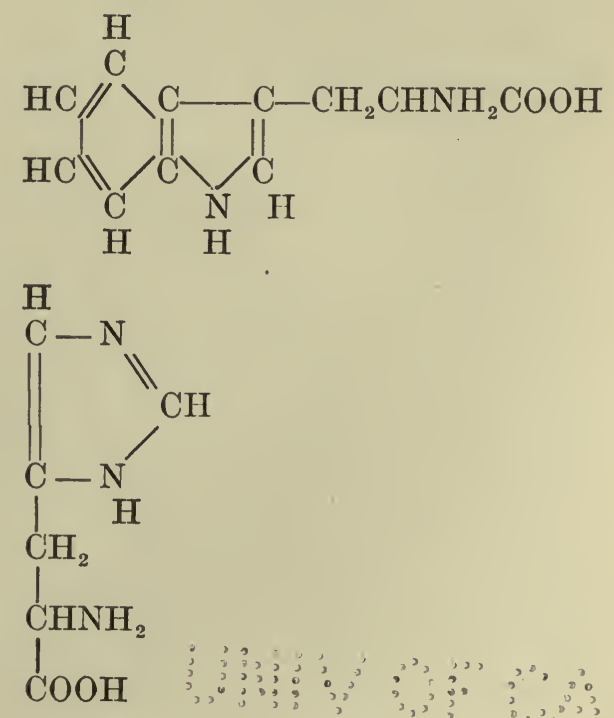
19. Proline<smiles>O=C(O)C1CCCN1</smiles>

20. Oxyproline

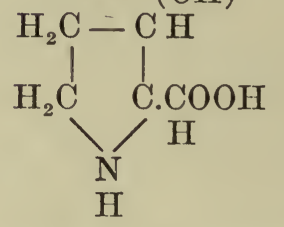

The position of the hydroxyl is uncertain.

General Properties and Reactions of Amino Acids.-Solubility, Taste, Optical Activity.-The amino acids obtained by hydrolysis of protein are crystalline substances, which are readily soluble in water, with the exception of cystine, which dissolves with difficulty in both cold and hot water, and of tyrosine, which is quite insoluble in cold water, but dissolves more readily in hot water. Solutions of monoamino-monocarboxylic acids are neutral in reaction. Solutions of dicarboxylic acids are acid, and of diamino acids are alkaline. The solutions of monoamino-monocarboxylic acids in reality have both acid and basic properties and should properly be classed as amphoteric. They all dissolve in dilute acids or alkalies, except cystin, which is not readily soluble in dilute ammonia. The amino acids vary in taste. Glycocoll, alanine and caprine are sweet, leucine is tasteless and isoleucine is bitter. With the exception of glycocoll, all the amino acids are optically active and exist in two forms, a dextro- and a levorotatory. Usually only one of the two is found as a protein constituent, and this is more often the levorotatory variety. If proteins are broken down by hydrolysis with alkali, the resulting amino acids are racemic, that is, they exist as equal amounts of the two optical isomers. The form of the acids not present in the protein is believed to be produced diaring the hydrolysis. Hydrolysis by 
enzymes, and to a certain extent by acids produces optically active acids, either dextro- or levorotatory, but does not cause racemization.

Acids, Bases.-The amino acids add on acids such as hydrochloric at the amino group. Thus

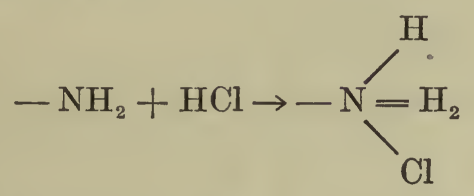

The acid raises the valence of the nitrogent to five. They also interact with bases to form salts.

\section{$-\mathrm{COOH}+\mathrm{NaOH} \rightarrow-\mathrm{COONa}+\mathrm{H}_{2} \mathrm{O}$}

At the amino group the amino acids add on the salts of certain metals such as cupric chloride, mercuric chloride, etc. This property is often made use of to precipitate amino acids.

Formaldehyde.-With formaldehyde, amino acids form methylene compounds. The basic properties of the amino group are thus greatly reduced, and the terminal carboxyl group can be titrated with a standard alkali. On this process a much used method for estimating amino acids is based (Sörensen's method).
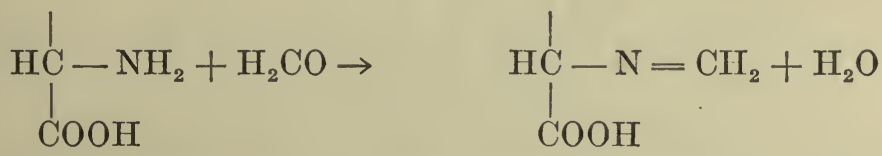

Carbamino Reaction.-The amino acids interact with carbon dioxide in the presence of calcium salts to form carbamino compounds. These have the following structure:<smiles>[R]C1NC(=O)O[13CH2]OC1=O</smiles>

If the nitrogen in this compound is determined, and also the amount of $\mathrm{CO}_{2}$ which is combined, a relationship between the 
amounts of nitrogen and $\mathrm{CO}_{2}$ may be established. In case the radicle $\mathrm{R}$ above contains no nitrogen $\frac{\mathrm{CO}_{2}}{\mathrm{~N}}=1$ for the above compound. If the radicle $\mathrm{R}$ contains nitrogen, however, as will be the case if the compound contains diamino acids, or is a substance known as a peptid, in which two or more amino acids are linked together, $\frac{\mathrm{CO}_{2}}{\mathrm{~N}}$ will be less than 1 .

Oxidation.-Oxidation of amino acids may yield a variety of products according to the strength of the oxidizing agent. The $\mathrm{NH}_{2}$ groups are not split off by acids or by alkalies to any extent. Alkalies, however, split arginine into ornithine and urea, and split off the sulphur from cystin and cystein. The small amount of ammonia given off in acid hydrolysis of protein is believed to come from the few acid amid groups $-\mathrm{CO}-\mathrm{NH}_{2}$ present. Oxidation with permanganate or hydrogen peroxide yields pyruvic acid from alanine. This compound is interesting since it is believed to be one of the steps in the breaking down of carbohydrates in the body, and thus may be a substance by way of which amino acids and monosaccharides can be converted into one another in the body. The substance has the formula<smiles>CC(=O)C(=O)O</smiles>

Nitrous Acid.-With nitrous acid the $\propto$ amino acids are broken down. Their nitrogen is liberated in the form of the free gas. This will be recognized as the familiar reaction of nitrous acid with primary amines.

$$
\mathrm{R} \mathrm{NH}_{2}+\mathrm{HONO} \rightarrow \mathrm{ROH}+\mathrm{N}_{2}+\mathrm{H}_{2} \mathrm{O}
$$

This reaction is the basis for the Van Slyke method for estimating amino acids, a method which has proved most useful in helping to settle some of the difficult questions with reference to the fate of the proteins in the body. From this brief re- 
view of some of the important facts relating to the amino acids we will turn our attention to the general properties and reactions of the proteins themselves.

\section{General Protein Reactions}

The general protein tests may be divided into two groups, the color tests and the precipitation tests.

Color Tests.-The protein color tests are by no means specific for proteins. They are tests which are given by certain groupings or certain constituents generally found in the protein molecule. If the grouping upon which a test depends is absent from a particular protein, that protein will not respond to the test in question. Thus a single positive test should not be taken as evidence of the presence of a protein, but should be confirmed by some other test.

Biuret Test.-If concentrated sodium hydroxide is added to a protein solution, and then a few drops of very dilute copper sulphate solution, a violet color appears either at room temperature or on slight warming. This test is named from the fact that it is given by a substance biuret which is made from two molecules of urea with loss of $\mathrm{NH}_{3}$

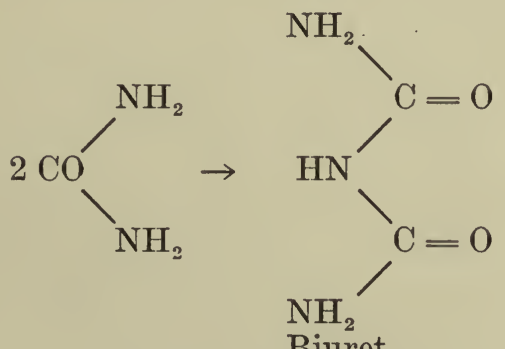

Biuret

This substance does not occur as a constituent of the protein molecule. The test depends on the presence of two amid groups - $\mathrm{CO}-\mathrm{NH}_{2}$ united either directly or by a carbon or nitrogen atom. One of the amino groups may be substituted as $-\mathrm{CO}-\mathrm{NHR}$ but not both of them. If the $\mathrm{NH}_{2}$ of the amid 
groups is split off by the action of strong acids, the proteins will no longer give a biuret reaction. Ammonium salts such as ammonium sulphate interfere with the test, and should be decomposed by boiling the mixture with strong alkali before making the test. Some of the proteins, e.g., the histones, and some of the products of protein hydrolysis give a reddish color.

Millon's Test.-If a few drops of Millon's reagent (a solution made by dissolving mercury in concentrated nitric acid) is added to a protein solution, a yellowish preciptate forms. On boiling, this precipitate turns rose pink. If the boiling is continued the pink color is destroyed and the precipitate turns brown. At times the whole solution becomes pink. If the protein happens to be insoluble, it will give the test quite as well, turning a very decided pink or red. The test depends on the presence in the protein of tyrosine, and is given only by those proteins which contain this amino acid. Phenol or any other compound which contains a hydroxyphenyl group will give the test, however. The dihydroxybenzenes do not give the test unless one hydroxyl group is substituted. Chlorides, alcohol or hydrogen peroxide will interfere with the test. Thus it is not serviceable to test urine for protein, since urine contains large amounts of sodium chloride.

Xanthoproteic Test.-If a protein is warmed with concentrated nitric acid the mixture turns lemon yellow. On the addition of alkali the color changes to a deep orange. The protein need not be in solution. Students of chemistry will recall having performed this test upon themselves by getting concentrated nitric acid upon the fingers, producing the familiar yellow spots. The skin is made of protein material. This test depends upon protein constituents containing the benzene ring, namely tyrosine, phenylalanine and tryptophane. The colored substance is a nitro derivative of benzene. Any substance, protein or otherwise, containing a benzene ring will respond to this test.

Adamkiewicz or Hopkins-Cole Test.-If glacial acetic acid, or a solution of glyoxylic acid (prepared by reducing oxalic 
acid with magnesium or sodium amalgam) is added to a protein solution, and concentrated sulphuric acid added so as to form a layer at the bottom of the test tube, a violet ring will form at the juncture of the two liquids. This test is due to the tryptophane in the protein molecule, and only those proteins containing this amino acid will respond to the test. When glacial acetic acid is used, the test is believed to be due to the presence as an impurity of glyoxylic acid, $\mathrm{HCO}-\mathrm{COOH}$, or other aldehydes. Nitrates, nitrites, chlorides, chlorates and some other substances interfere with this test.

Sulphur Test.-If concentrated sodium hydroxide and lead acetate are added to a protein solution and the mixture boiled, a brown or black color appears. The unoxidized sulphur of the cystein or cystin is split off and combines with the lead to form the dark brown or black lead sulphide.

Precipitation Reactions.-Proteins may be precipitated by a variety of reagents. The behavior of protein solutions with precipitation reagents, and in fact many other properties of protein solutions indicate that the proteins do not form true solutions such as that of sodium chloride in water. They form colloidal solutions.

Heat.-Many proteins are precipitated by heat. A slightly acid reaction, and the presence of salts is desirable if precipitation is to be complete. Alkaline solutions of proteins do not precipitate on boiling. Neutral solutions, especially if salts have been removed by dialysis, will precipitate only imperfectly. Solutions of some proteins, i.e., casein, gelatine and secondary derived proteins such as proteoses and peptones do not precipitate on boiling. In general for each protein there is a specific precipitation temperature, but experimental conditions such as the amount of salts present, the rapidity of heating and other factors eause rather wide variations in the precipitation temperature. A protein precipitated by boiling in weak acid solution cannot readily be redissolved. Some as yet unknown change has taken place in the protein molecule, and the sub- 
stance is said to be coagulated. Proteins may be coagulated also in other ways.

Mineral Acids.-Proteins are precipitated by the addition of small amounts of the strong mineral acids,-hydrochloric, sulphuric and nitric. The precipitate dissolves in excess of the acid, particularly if the solution is heated. Glacial acetic acid does not precipitate proteins. There has been much discussion of the nature of this precipitation. Probably the proteins are thrown down in the form of salts. Precipitation with concentrated nitric acid is often used as a test for proteins. To the solution to be tested, concentrated nitric acid is added carefully so that it will form a layer at the bottom of the test tube. In the presence of protein a cloudy ring appears at the juncture of the two liquids.

Salts.-Proteins are precipitated by salts. The salts of heavy metals, such as copper, iron, mercury, lead, etc., will throw down the proteins from their solutions. The precipitates formed are in many cases true salts of the protein and the metal. Where this is the ease, the precipitation takes place best usually in a weakly alkaline solution, for in this condition the proteins are negatively charged, and will combine with the positive metal ions. Some proteins, such as protamines and histones, which have large amounts of diamino acids, form alkaline solutions and the protein carries positive charges. More alkali must be added to give these proteins negative charges than in the case of albumins. Casein, which contains much dicarboxylic acid (glutamic) carries negative charges (since it gives off hydrogen-ions to the water). Casein may thus be precipitated by metals even in slightly acid solution.

The conditions governing precipitation of proteins by metals are somewhat complicated by the fact that some metals such as mercury, gold, copper, and others (these metals have a lower solution tension than hydrogen) combine with the amino group also, so that salts of these metals will precipitate proteins in weakly acid as well as in weakly alkaline solution.

Three salts much used to precipitate proteins are ammonium 
sulphate, magnesium sulphate and sodium chloride. High and varying concentrations of these salts are necessary to throw down the different proteins. By the use of suitable amounts of these salts some of the protein groups may be separated from others. The process is known as "salting out." The proteins are not coagulated, and may be redissolved on removal of the salt.

Alkaloidal Reagents.-Many compounds known as alkaloidal reagents will precipitate proteins. Among these are several acids such as tannic, picric, phosphotungstic, phosphomolybdic, ferrocyanic, chromic, and dichromic. The precipitates undoubtedly are compounds of protein with the negative-ion of the precipitating reagent. The tests are thus carried out to best advantage in weakly acid solution. Exceptions to this statement depend upon conditions similar to those discussed under precipitation by the addition of salts.

Tungstic acid as a protein precipitant is of particular interest, since it is used to precipitate the proteins of the blood in the preparation of the protein-free blood filtrate used in the Folin and $\mathrm{Wu}$ system of blood analysis. Sodium tungstate is added to the diluted blood, and then sulphuric acid. Tungstic acid is set free, and precipitates the blood proteins completely, so that the filtrate is water-clear, and free from all protein.

Alcohol.-Alcohol in sufficient concentration will precipitate many of the proteins. Some few are soluble in alcohol, however. If the precipitate is allowed to stand in the alcohol, it will become coagulated, and cannot be redissolved on removal of the alcohol.

\section{Structure of the Protein Molecule}

Since proteins make up so large a part of living tissue, and are indispensable to life it would be of great interest to find out, how the protein molccule is constructed. This problem has been studied by some of the foremost biochemists for a long time, and although the formula for a protein is still unknown, much is now known as to the manner in which the parts of a protein 
molecule are put together. On hydrolysis, proteins yield a mixture of amino acids. These, then, must be the building stones out of which the proteins are constructed. How are these building stones put together? Much evidence has accumulated to show that the amino acids are joined together in long chains, the different units being linked by what is known as the amid or "peptid" linking,- the union, with loss of water, of the carboxyl group of one acid with the amino group of another.

\section{$\mathrm{NH}_{2} \mathrm{CH}_{2} \mathrm{COOH}+\mathrm{NH}_{2} \mathrm{CH}_{2} \mathrm{COOH} \rightarrow$ $\mathrm{NH}_{2} \mathrm{CH}_{2} \mathrm{CO}-\mathrm{NH}-\mathrm{CH}_{2} \mathrm{COOH}+\mathrm{H}_{2} \mathrm{O}$}

A compound of this type is called a peptid,-if made from two amino acids, a dipeptid, if from three amino acids, a tripeptid, if from many amino acids, a polypeptid. On partial hydrolysis of proteins, such compounds actually have been found in the resulting mixture, and have been shown to be identical with compounds of known structure built up in the laboratory. The most complex compound of this nature yet synthesized was prepared by Emil Fischer. He prepared a substance having eighteen amino acids in the chain, thus an octadecapeptid. This compound had a molecular weight of 1213 , and from its properties, was still much simpler than a protein. The synthesis of such compounds is extremely laborious and expensive, so that no attempt has been made to earry the process further. One of the factors tending to increase the labor of synthesis is the fact that the amino acids used are optically active. As ordinarily obtained, they consist of equal portions of the dextroand levorotatory forms, and must be separated into the two varieties. The method most used to accomplish this consists in preparing the salts of the two forms with some optically active base, such as brucine, cinchonine; etc. The brucine compounds of the d-and l-forms may be separated since they usually vary considerably in solubility, and on concentration one or the other will erystallize out first. A second method consists in allowing various microorganisms to act on the mixture of the two optical isomers. Usually one form will be destroyed 
by the microorganism, the other to a much smaller degree, or not at all.

The optically active acids then may be built up into peptids at will. Three methods are in use to effect this synthesis.

1. Ester method.-

Glycocoll ester is converted into a ring compound

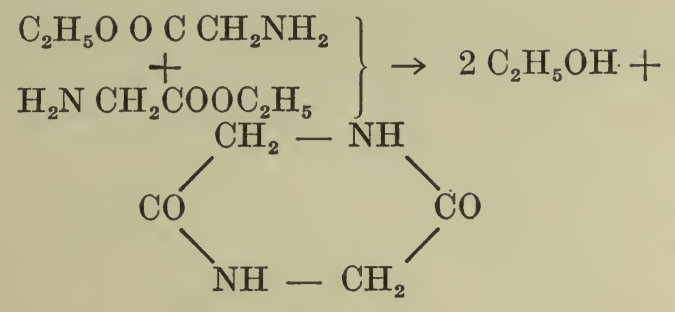

Under the action of alkali this ring compound is split open, forming a dipeptid

\section{$\mathrm{NH}_{2} \mathrm{CH}_{2} \mathrm{CO} \mathrm{NH} \mathrm{CH}_{2} \mathrm{COOH}$}

Of course the amino acids used may be varied, and thus different dipeptids obtained. The method serves only to build up dipeptids, however, and if two different amino acids are used it has certain other disadvantages.

2. Synthesis by means of acid chlorides.-

If glycocoll is treated with chloracetyl-chloride the following reaction takes place:

\section{$\mathrm{ClCH}_{2} \mathrm{CO} \mathrm{Cl}+\mathrm{NH}_{2} \mathrm{CH}_{2} \mathrm{COOH} \rightarrow \mathrm{HCl}+$ $\mathrm{Cl} \mathrm{CH}_{2} \mathrm{CO}-\mathrm{NH} . \mathrm{CH}_{2} \mathrm{COOH}$}

By the action of ammonia, the chlorine atom may be replaced by $\mathrm{NH}_{2}$, and a dipeptid results.

$$
\mathrm{NH}_{2} \mathrm{CH}_{2} \mathrm{CO}-\mathrm{NH} \text {. } \mathrm{CH}_{2} \mathrm{COOH} \text {. }
$$

This may be treated with chloracetyl chloride again, and in a manner perfectly analagous to the first reaction, a tri-peptid or higher peptid built up. Here again various amino acids, and various halogen derivatives may be used. The limitation of this 
method lies in the fact that the chain can be extended only at one end.

3. By treating an amino acid or a peptid with phosphorus pentachloride, the carboxyl group is converted into an acid chloride group which then may be caused to interact with the amino group of an amino acid or a peptid in a manner analagous to that described in the second method of synthesis. Thus glycocoll will be converted into $\mathrm{NH}_{2} \mathrm{CH}_{2} \mathrm{CO} \mathrm{Cl}$ which may be combined with a second glycocoll molecule or other amino acid to form a dipeptid as in 2 .

The compounds prepared in these ways show many of the reactions characteristic of the products of partial hydrolysis of proteins,- - thus some give the biuret reaction, those containing tyrosine give the Millon test, those containing tryptophane give the Adamkiewicz test, and certain of the more complex of them are precipitated by some of the protein precipitants. Many of them even are split by enzymes found in the body which are known to split protein decomposition products. These synthetic products thus are identical with those obtained in protein hydrolysis, a fact which is one of the strongest pieces of evidence that the proteins are constructed on the general plan of the polypeptids. Other evidence for this form of linkage in the protein is given by various facts. The proteins react only to a very limited extent with reagents which interact with free $\mathrm{NH}_{2}$ groups. Very little hydrochloric acid is taken up by them, the formaldehyde reaction described above shows relatively few free amino groups, as does also the Van Slyke method also described above. In alkaline hydrolysis the amino acids become racemic for the most part. Only those whose carboxyl groups are combined, as is the case in the amid linking, will become racemic on hydrolysis. All these results are explained if the amino acids making up the proteins are assumed to be united by the "peptid linking."

The behavior of polypeptids with enzymes is especially interesting, and demonstrates the extremely specific character of enzyme action. For example, it has been shown that the di- 
peptid d-alanyl-d-alanine is split by pancreatic juice, but not d-alanyl-l-alanine. In general, those peptids made up of the optical form of the amino acids which is found in nature will be split by enzymes. Peptids made from the optical isomers which do not occur in nature are not split, or are split less readily by enzymes.

From a study of the polypeptids it has been learned that the ease with which a given polypeptid may be precipitated from solution depends not only on the size of the molecule, but also upon the particular amino acids present in it. Thus a polypeptid containing tyrosine, tryptophane or cystin will precipitate at a much lower concentration of a given precipitating reagent than a second polypeptid of equal molecular weight, containing none of these acids. Many polypeptids, both those prepared synthetically and those obtained from protein hydrolysis will give characteristic protein reactions, though no polypeptid has been prepared which closely approaches the proteins in the complexity and size of its molecule.

On hydrolizing a protein, that is, breaking it down into simpler products, the process evidently is not a symmetrical division and redivision of the molecule. In tryptic digestion tyrosine, tryptophane and cystine are split off much quicker than alanine, leucine and valine, whereas glycocoll, proline and phenylalanine are not split off even after prolonged digestion. We must assume, therefore, that certain groups or "building stones," are split off from the great protein molecule, which is thus reduced through various stages to proteoses, peptones and in complete hydrolysis to the amino acids.

Putrefaction of Proteins.-When proteins are broken down by the action of bacteria in the intestines, products are formed some of which are very injurious to the organism. The amino acids produced by the hydrolysis of the proteins are attacked and partially broken down in a variety of ways. From tryptophane by the removal of its side chain, skatol and indol are produced. 


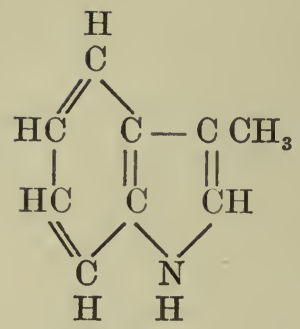

Skatol

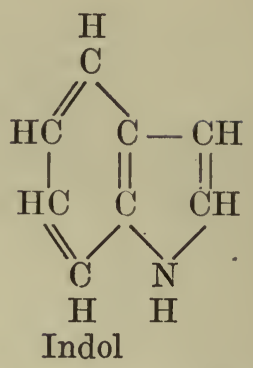

From amino acids, by removal of $\mathrm{CO}_{2}$ from the carboxyl group various amines are produced, e.g., from tyrosine, tyramine

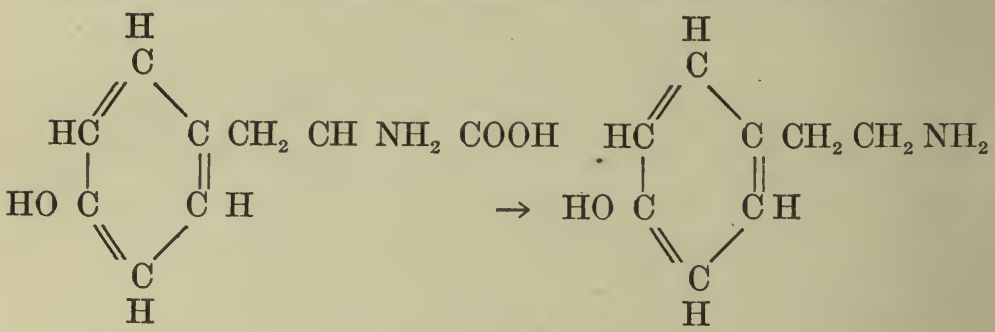

From histidine, histamine is produced in a similar way. Such compounds are known by the name "ptomaines," and they are extremely toxic. Cystine and cystein give rise to hydrogen sulphide and other sulphur compounds. A condition of constipation, in which intestinal contents are retained unduly long in the body, and putrefaction thus prolonged, favors the production of such substances. On absorption into the blood they give rise to headaches, general debility and in time, to more serious disorders. Undoubtedly certain compounds of this type are produced normally by the enzymes of the tissue cells themselves, and it is probable that some of them are of importance for the regulation of certain body processes. Adrenaline is such a substance.

\section{Individual Groups. Simple Proteins}

The important characteristics of the proteins as a class have been considered. Let us now review the properties of groups and of individual proteins. 
Albumins. - The albumins are found widely distributed in nature. Serum albumin in the blood, ovalbumin in egg-white, lactalbumin in milk and myogen in muscle are the best known members of this group. Compounds resembling the albumins have been found in plants, although they differ in some of their properties from the animal albumins. Albumins contain no glycocoll, and relatively much sulphur (about 1.6-2.2\%). They are soluble in water and dilute salt solutions, are precipitated by strong mineral acids, by saturation with ammonium sulphate in neutral solution and by many other precipitants. They are not precipitated by saturating a neutral solution with magnesium sulphate or sodium chloride, but if such a solution is acidified, the albumins precipitate. Albumins in solution coagulate on boiling, if salts are present and if the solution is faintly acid.

Globulins.-Globulins also are widely distributed in nature, both in animals and in plants. They often are associated with albumins. Important members of the group are serum globulin, fibrinogen and its derivative fibrin of the blood, and myosin of the muscles, ovoglobulin in eggs, lactoglobulin in milk, neuroglobulin in nerve tissue, and several plant globulins such as edestin from hemp seed, legumin from peas, lentils, etc., and various others from nuts or other materials.

The globulins are insoluble in pure water, and they may be precipitated by pouring a solution of a globulin into a large volume of pure water, or by dialyzing out the salts against distilled water through a parchment membrane. Globulins also differ from albumins in containing glycocoll, and in the greater ease with which they precipitate on the addition of a neutral salt. Thus they are precipitated by half saturation with ammonium sulphate or by saturation with magnesium sulphate or sodium chloride in neutral solution.

Fibrinogen has the property of clotting or coagulating, and is responsible for the clotting of the blood. If freshly drawn blood is stirred or beaten, the fibrinogen collects in stringy masses called fibrin. If washed free from corpuscles fibrin is a white, 
tough, elastic material which gives the usual protein tests. Myosinogen and myosin are the chief proteins of muscle tissue.

Glutelins.-The glutelins are proteins found only in the the seeds of plants. The important members of the group are glutenin from wheat, oryzenine from rice, and avenine from oats. There probably are others. They are characterized by being insoluble in pure water, salt solutions or alcohol. They dissolve in dilute acid or alkali.

Prolamines.-The prolamines are proteins found only in the grains. They have been obtained from all grains except rice. The best known members are gliadin from wheat, zein from corn, and hordein from barley. The group was named because of their high content of proline. The prolamines are characterized by being soluble in 70-80\% alcohol. They are insoluble in water. They contain only small amounts of arginine and histidine and no lysine.

Albuminoids.-Albuminoids are proteins obtained from a wide variety of sources in the animal world. Important members of the group are keratin from horn, nails, hair, hoofs, etc., collagen from connective tissue and bone, its derivative gelatine, which properly does not belong in this class however, and elastin, from ligaments and connective tissue. There also are various other albuminoids. The members of the group are classed together for convenience, since they are insoluble in water and most protein solvents, and are constituents of protective or supporting portions of the body. Keratin is the chief constituent of hair, horn, nails, feathers, the epidermal layer of the skin, etc. A keratin also has been obtained from the brain and nerve tissue. There probably are several keratins. The keratins contain much sulphur (1-5\%). They give the characteristic protein color tests.

Collagen is the chief constituent of the connective tissue and the chief organic constituent of bone. It occurs also in cartilage. There probably are several collagens. Collagen, if boiled with water or dilute acid, is converted into gelatine. Collagen is insoluble in water, dilute salt solutions, dilute acids and alkalies. 
Gelatine, obtained by boiling collagen, swells up in cold water, but dissolves in hot. If sufficiently concentrated, such a solution will set, on cooling, to a jelly. Gelatine is not coagulated by boiling, nor will it precipitate on the addition of strong mineral acids or metallic salts. It may be precipitated by a variety of reagents, however, such as alcohol, tannic acid, etc. Gelatin's gives the biuret reaction, but it contains neither tyrosine nor tryptophane, and thus if pure will not give the Millon or Hopkins-Cole tests. Gelatine contains much glycocoll. After prolonged boiling, a gelatine solution will no longer gel on cooling.

Elastin.-Elastin occurs in connective tissue, and in largest amount in the cervical ligament. Fresh elastin forms yellowish shreds or strings which are elastic in character. It is insoluble in water and most of the protein solvents. Elastin contains large amounts of glycocoll and leucine. It does not give the Hopkins-Cole test.

Histones.-The histones are found chiefly in the spermatozoa of fish, but the globin portion of the hemoglobin of the blood is usually classed in this group. Their chief characteristic is a high percentage of diamino acids. In this respect they stand midway between the protamines and the remaining simple proteins. They are basic in nature. The group is not sharply defined.

Protamines.-The protamines are the most basic of the proteins. They occur in the ripe spermatozoa of fish. They are named from their origin, as salmine, from the salmon, sturine from the sturgeon, etc. They are characterized by their high percentage of diamino acids, particularly arginine. Salmine contains $87 \%$ of arginine. As a result, solutions of protamines in water are alkaline in reaction. They give the biuret test, but most of them do not give the other color tests for proteins. They are precipitated fairly well by neutral. salts and are not coagulated by boiling.

\section{Conjugated Proteins}

The members of this group are made up of protein combined with some other non-protein substance, which is called the pros- 
thetic group. Among the conjugated proteins are substances of great biological importance.

Glycoproteins.-The glycoproteins are compounds which on breaking down yield a protein and a relatively high percentage of a carbohydrate or carbohydrate derivative. Other proteins also yield carbohydrates on hydrolysis, but in smaller amounts than the glycoproteins. The group is divided into two divisions, mucins and mucoids. They are very difficult to purify, especially the mucins, since they are slimy in character, and as a result there is much disagreement as to their composition and properties. Mucin is secreted by the salivary glands, by certain mucous membranes and elsewhere. The skin of some of the lower animals secretes large quantities of mucin. The mucins are distinguished from the mucoids by their slimy character and by the fact that on precipitation with acetic acid, they do not redissolve in excess of acid. The mucoids are found in tendons, in cartilage, in the cornea and crystalline lens, in egg white and various other places. Some authors divide this group again into mucoids and chondroproteins. The mucoid of tendon or cartilage may be extracted with lime water. On acidifying with acetic acid, the mucoid precipitates, but it will redissolve in excess. Both the mucins and the mucoids contain a relatively high amount of sulphur, a part of which, at least, is in the form of oxidized sulphur. On hydrolysis the mucoids yield a substance chondroitic acid or chondroitin sulphuric acid, which has been the subject of much study. The carbohydrate radicle in the glycoproteins is usually glucosamine or galactosamine.

Phosphoproteins.-The phosphoproteins are compounds of importance because they furnish a large share of the protein nourishment of the growing young of animals and birds. There are two important members of the group, the casein of milk and the vitellin of egg yolk. They are characterized by containing a relatively high amount of phosphorus (about 1\%) which is present in some form neither lecithin nor nucleic acid. On digestion, the phosphoproteins leave a difficultly digestible residue known as pseudonuclein. The phosphoproteins are acid in 
character. In their solubilities and precipitation properties they resemble both the globulins and the nucleoproteins. They may be distinguished from the former by their phosphorus content, and from the latter by the fact that they yield no purine bases on hydrolysis. Cow's milk contains about $3-4 \%$ casein, human milk about $0.5-1.5 \%$. Casein is not coagulated by boiling. It is insoluble in water, but dissolves readily in dilute alkalies. From such a solution, and from milk, casein is precipitated by the addition of a small amount of acid. This occurs also in the souring of milk. Bacteria decompose the lactose, forming organic acids. From this acid solution the casein is precipitated, causing the characteristic clotting of sour milk. It may be prepared by diluting milk, and adding a small amount of acetic acid. To free the casein from fat it may be redissolved in sodium carbonate and reprecipitated with acid. This process should be repeated several times if a pure product is desired. Casein contains no glycocoll, but little cystine and relatively much tryptophane. On hydrolysis it yields also several acids which are not obtained from other proteins. Casein is elotted by a ferment, rennin, which occurs in digesive juices, particularly the gastric juice. It may be well to state in this connection that the nomenclature of casein and its allied substances is somewhat confused. By many investigators the substance as it occurs in milk is called caseinogen. This is converted by rennin into another substance, paracasein. Paracasein differs from caseinogen at least in one respect, the insolubility of its calcium salt. The calcium salt of caseinogen is soluble, that of paracasein insoluble. Since calcium salts are found normally in milk, paracasein is precipitated as the familiar curdy material. If calcium is previously removed by adding an oxalate to the milk, the paracasein is formed from caseinogen by rennin, but will not precipitate. Subsequent addition of excess of a soluble calcium salt will cause precipitation even if the milk has been boiled to destroy the rennin. In the transformation of caseinogen into paracasein a protein like substance called albumose, or "whey protein" is split off. This clotting is the first step in the digestion 
of casein in the stomach, and serves to prevent the milk from passing on too quickly into the intestine.

Vitellin.-Vitellin is found in egg yolk and may be prepared by extracting the yolk with ether to remove lecithin, cholesterol, etc. The residue is dissolved in salt solution and reprecipitated by pouring into a large volume of water. To remove the last of the lecithin it is necessary to extract with boiling alcohol.

Hemoglobins.- This group often is called the group of chromoproteins, since its members usually are colored substances. The most important of them is hemoglobin or oxyhemoglobin, the red coloring matter of the blood. This compound is contained in the red corpuscles in higher animals. In some of the lower animals it is simply dissolved in the blood plasma. It, or a substance closely allied to it, occurs also in red muscle. The hemoglobin fulfils an important function in the body, that of transporting oxygen from the lungs to the tissues. If hemoglobin is exposed to a plentiful supply of oxygen, as is the case when the red corpuscles are passing through the capillaries surrounding the tiny air spaces, the alveolæ in the lungs, it takes up oxygen and is converted into oxyhemoglobin. When the corpuscles containing this oxyhemoglobin reach the tissues, again they pass through a fine network of capillaries. Here, however, the oxygen supply is very low, as oxygen is rapidly used up by the cells in oxidation processes. The oxyhemoglobin gives up its oxygen, becoming hemoglobin again, and the oxygen passes out through the capillary walls to the region of low oxygen supply. Returning to the Iungs, the hemoglobin takes up a fresh supply of oxygen, and again carries it to the tissues. This property of hemoglobin may be demonstrated easily as follows: On adding an equal volume of water to the blood, the hemoglobin diffuses out of the corpuscles and the solution becomes clear. The blood is said to be "laked." If the mouth of the test tube is closed with the thumb or a stopper and the liquid shaken well, the color of the solution becomes a brilliant red. It now contains oxyhemoglobin, the color of which is a much brighter red than that of hemoglobin. this substance gives arterial blood its bright 
red color. If a mild reducing agent is added to this oxyhemoglobin the color becomes much darker. The oxygen has been taken away from the oxyhemoglobin, which has now become hemoglobin. This substance gives venous blood its dark red color. This process may be repeated indefinitely. A convenient mild reducing agent is Stokes' fluid which contains $2 \%$ ferrous sulphate, $3 \%$ tartaric acid and ammonia sufficient to redissolve the precipitate which first forms on adding this reagent. The amount of oxygen which can be carried by a given amount of blood varies somewhat. On the average from 100 c.c. of fully oxygenated blood 19-20 c.c. of oxygen can be obtained (measured at $0^{\circ} \mathrm{C}$. and $760 \mathrm{~mm}$ of mercury pressure). A portion of this is simply dissolved in the blood plasma, but most of it is combined with hemoglobin. The tissues do not remove all of the oxygen from the blood, for venous blood still contains considerable oxyhemoglobin. From 100 c.c. of venous blood an average of 15 c.c. of oxygen may be obtained, although this value varies considerably. The molecular weight of hemoglobin is very large, probably about 16,000 , and one molecule of hemoglobin is believed to combine with one molecule of oxygen $\left(\mathrm{O}_{2}\right)$. The air normally contains about $20 \%$ oxygen. This percentage may be much lowered, however, before the amount of oxyhemoglobin in a hemoglobinnxyhemoglobin mixture will be greatly diminished. If the per cent of oxygen is reduced to $13 \%$ of an atmosphere the mixture will still contain $93 \%$ oxyhemoglobin and only $7 \%$ hemoglobin.

The dissociation of oxyhemoglobin is favored by the presence of salts, carbon dioxide, and lactic acid. In the presence of any of these substances, or if their amounts are increased, oxyhemoglobin tends to give up some of its oxygen. The biological importance of this fact is evident. In the tissues, the production of carbon dioxide or lactic acid will aid in causing oxyhemoglobin to yield up its oxygen, which is then used in the various oxidation processes going on in the cell.

Hemoglobin may be split into two parts, globin, a protein, perhaps a histone, and hemochromogen. From oxyhemoglobin hematin is obtained in place of hemochromogen. Globin makes 
up about $95 \%$ of the hemoglobin, hemochromogen about $4 \%$. The corpuscles contain about $30 \%$ of hemoglobin, an amount much greater than could be dissolved in an equal amount of fluid, so it must be assumed that hemoglobin is held in some loose combination with the other constituents of the corpuscles. Hemoglobin and many of its derivatives contain iron to the extent of about $3 \%$. This iron seems to be directly connected with the ability of hemoglobin to combine with oxygen.

Oxyhemoglobin may be erystallized with comparative ease. Oxyhemoglobins from different animals form erystals of greatly varying form, and may be crystallized with varying degrees of ease. Those easiest to crystallize are oxyhemoglobin of the guinea pig, which forms tetrahedra, of the rat (rhomboids), of the squirrel (hexagons), of the horse, dog, etc. Oxyhemoglobin from man (long rods, rhomboids), ox, and other animals may be obtained in erystalline form, but with greater difficulty. Crystals of the types which crystallize easily may be obtained by a variety of methods. That of Reichert consists in adding $1-5 \%$ of solid ammonium oxalate to blood, either before or after laking or defibrinating. A drop of this blood placed on a slide will crystallize quickly. On standing in the ice chest very beautiful large crystals form.

Reichert and Brown have studied the oxyhemoglobin crystals from the blood of a great many animals and maintain that biological relationships may be traced in the crystal form, since oxyhemoglobins from animals of the same species erystallize in similar forms. Hemoglobin is more soluble than oxyhemoglobin, and is thus much more difficult to erystallize.

Detection of Hemoglobin.-In medical practice, and also in medico-legal cases it often is of the greatest importance to detect and identify hemoglobin or its derivatives. This is done in a variety of ways as follows.

Catalytic Activity of Blood.-If hydrogen peroxide is added to blood, or even to blood diluted to the point. where the solution is no longer colored, the peroxide is decomposed, and bubbles of oxygen gas are given off. If the blood is first boiled, no action 
takes place. Thus it appears that the blood contains an enzyme capable of decomposing peroxides.

Hemin Test.-One of the best tests for blood is the preparation of hemin crystals. Hemin is the hydrochloride of hematin. The hemin test will not differentiate between the blood of man and other animals. Although hemoglobins from different animals differ, it is the globin portion which varies, and the hematin from different animals is undoubtedly the same. The hemin test is very delicate. The suspected stains are extracted with water or weak alkali, the solution evaporated to dryness and the residue used for the test. If the solution is very dilute, the pigment may be precipitated with tannic acid, and the test made on the precipitate.

The test is performed by evaporating a drop of blood or suspected material to dryness on a slide, adding sodium chloride and a few drops of glacial acetic acid. The mixture is covered with a cover glass and heated carefully until the acid boils. On cooling, brown or reddish brown rhomboidal crystals of hemin form. If crystals do not form the first time, the mixture should be boiled again as above. It may be necessary to repeat the process several times. As hematin is very stable, blood stains which have putrefied, or which are even centuries old still will give the test. Wood smoke and swamp water do not destroy hematin; it may be destroyed if certain moulds have grown on the stain however. It is of interest that the excreta spots of blood sucking insects will give a positive hemin test.

Guaiac Test.-Benzidine Test.-Blood has the property of transferring oxygen from hydrogen peroxide to easily oxidized chemical substances such as guaiac, benzidine and a variety of other compounds. On adding a small amount of a freshly prepared $1 \%$ alcoholic gum guaicum solution to a solution containing blood, and then a small volume of $3 \%$ hydrogen peroxide, a bluish green color develops. Old turpentine which has not recently stood in direct sunlight may be used in place of the hydrogen peroxide. In performing the benzidine test it is best to dissolve in glacial acetic acid the amount of benzidine which 
can be taken on a knife point, then add an equal volume of hydrogen peroxide and the liquid to be tested. If blood is present, the color produced will be a greenish blue. If blood has been diluted 200,000 times it still will give a very definite positive reaction. Other substances also respond to these tests, such as milk, living matter, etc. A slice of raw carrot gives a very good test. Milk, and living matter, (carrot) if boiled, no longer will give the test, whereas blood gives it quite as readily after boiling. Evidently in the case of blood the material responsible for the test is not an enzyme. As negative tests, these color tests may be taken to indicate the absence of blood. As positive tests, however, they are not conclusive without further confirmation from other tests. Also they do not distinguish human blood from that of other animals.

\section{Absorption Spectra of Oxyhemoglobin and Hemoglobin.-} Hemoglobin and many of its derivatives show characteristic absorption spectra. Thus a spectroscopic investigation often is of the greatest value in detecting blood in feces, urine, gastric contents or in stains in medico-legal work. The student is referred to the discussion of absorption spectra under pentoses in the chapter on carbohydrates. The nature of the absorption bands depends not only upon the substance present, but the concentration of the solution and thickness of the layer through which the light passes. Blood diluted ten times with water, and observed with a spectroscope in a flat sided cell about one centimeter in thickness allows only a little red light to pass through. If the solution is diluted however, it shows two absorption bands near together between the D and E Frauenhofer lines. On further dilution these lines become fainter and at sufficient dilution, finally disappear.

If Stokes' reagent is added to an oxyhemoglobin solution, thus converting the oxyhemoglobin into hemoglobin the double bands give place to a single continuous band in about the same location.

The amount of hemoglobin or oxyhemoglobin in blood or other fluids may be estimated by various means, the most con. 
venient consisting in comparing blood or diluted blood with a scale giving reds of different intensities corresponding to definite concentrations of hemoglobin.

\section{Derivatives of the Hemoglobins.-Carbon Monoxide-Hemo-} globin.-Hemoglobin forms compounds with various gases other than oxygen such as carbon monoxide, carbon dioxide, etc. Carbon monoxide, which is a constituent of illuminating gas combines with hemoglobin in molecular proportions. Its union apparently is firmer than that of oxygen, and apparently it combines with hemoglobin at the same place as does oxygen, for if both gases are present, carbon monoxide hemoglobin is formed, and the taking up of oxygen is interfered with. The carbon monoxide may be removed, however, by passing a stream of air through the liquid for some time, and oxyhemoglobin will be formed. In cases of asphyxiation by illuminating gas, carbon monoxide is found in the blood, thus preventing the proper transportation of oxygen to the tissues.

Solutions of carbon monoxide hemoglobin are a bright cherry red. The absorption bands look much like those of oxyhemoglobin,- - two dark bands between the $\mathrm{D}$ and the E lines. They are slightly nearer the violet end of the spectrum however, and on adding Stokes' reagent to the mixture they do not, as does the hemoglobin spectrum, give place to a single broad band.

Carbon dioxide apparently combines with hemoglobin at a point different from that at which oxygen combines, as combining with one does not prevent hemoglobin from combining with the other also.

Methemoglobin.-Methemoglobin is a compound derived from oxyhemoglobin. It contains the same amount of oxygen as oxyhemoglobin, but the oxygen is more firmly united than in oxyhemoglobin. Methemoglobin is found in the blood after poisoning with chlorates, amyl nitrite, etc., and is found occasionally in the urine, in transudates, cystic fluids, and elsewhere. Outside the body it may be prepared for study by adding fresh potassium ferricyanide solution, permanganate or other substances to oxyhemoglobin solutions. The solution turns a muddy 
brown. On dilution and observation with the spectroscope, a dark absorption band between the $\mathrm{C}$ and $\mathrm{D}$ lines is observed. Two fainter bands in the position of the oxyhemoglobin bands are considered by some investigators to be due to the presence of a small amount of this latter pigment. On adding Stokes' reagent to a methemoglobin solution, the substance is changed first into oxyhemoglobin, and this into hemoglobin, with corresponding changes in the spectrum.

On adding an alkali to a methemoglobin solution, alkaline methemoglobin is formed, which gives a characteristic spectrum of its own.

Acid Hematin.-Hematin is the compound into which oxyhemoglobin may be split by the action of acids or other agents. It contains the iron of the hemoglobin molecule, and is comparatively simple in structure. It has been shown to contain four pyrrol rings.

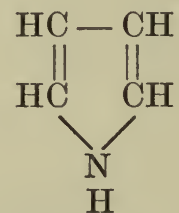

Pyrrol

Hematin is formed from oxyhemoglobin by the action of gastric and pancreatic juices. It is found thus in the feces after gastric or intestinal hemorrhage, but also, of course, after a meal of bloody meat. Hematin gives the brown color to cooked meat. It may be prepared by adding blood to glacial acetic acid and ether. The absorption spectrum of acid hematin is variable with the kind of acid used in its preparation and other conditions. If prepared as above, however, it shows a sharp dark band between the $\mathrm{C}$ and $\mathrm{D}$ lines and a fainter broad band between the $\mathrm{D}$ and $\mathrm{F}$ lines. Alkaline hematin shows characteristic bands.

Hemochromogen.-Hemochromogen, or reduced alkaline hematin is obtained by splitting off the globin from hemoglobin by the action of acids or alkalies, or by the reduction of alkaline hematin with Stokes' reagent. Like hematin, it also contains 
iron. Oxygen forms with hemochromogen a more stable compound than with hemoglobin. Hemochromogen shows characteristic absorption bands.

Hematoporphyrin.-Hematoporphyrin is an iron free derivative of hemoglobin which may be prepared by adding blood to concentrated sulphuric acid. The liquid becomes purple and may be shown to contain hematoporphyrin. It gives two absorption bands, one on each side of the $\mathrm{D}$ line. If the solution is made alkaline, the acid hematoporphyrin is changed to alkaline hematoporphyrin, which shows a different spectrum. Hematoporphyrin is found in the urine in considerable amounts after sulfonal poisoning and the statement has been made that small amounts occur in normal urine. Hematoporphyrin is interesting from the fact that it is closely related to phylloporphyrin, a derivative of chlorophyl, the green coloring matter in plants. In this same connection it is also interesting that hematoporphyrin acts as a photo-sensitizer, making animals sensitive to light. If hematoporphyrin is injected into a white mouse, no ill effects appear so long as the animal is kept in the dark. If exposed to strong sunlight, however, it will develop dyspnea, swelling of the ears, edema of the skin, and ultimately will die. In man, in some skin diseases connected with the action of strong sunlight, hematoporphyrin has been observed in the urine.

Fate of blood pigment in the body. Hemoglobin from broken down corpuscles is converted into bile pigments, and thus is the source of these coloring matters. The transformation in mammals is made in the liver itself and undoubtedly to a considerable exterit in the spleen, although this latter organ probably carries out only the first stages of the process. The bile pigments, bilirubin and biliverdin, and also urobilin, a yellow pigment in the urine thus come from the broken down hemoglobin of the blood.

Nucleoproteins.-The nucleoproteins are found in the nuclei of all cells, both plant and animal. It has been suggested that they occur in other portions of the cell, or in the blood plasma, but this is doubtful, although their decomposition products may occur there. Since the cell nucleus is intimately concerned in the 
process of cell division, and thus in reproduction and growth, any substances found regularly and characteristically in the nucleus will be of great interest. The nucleoproteins are characterized by containing nucleic acid, which may be obtained from them by splitting off the protein portion of the molecule. This process can be carried on in two stages, part of the protein splitting off more easily, leaving a product called nuclein, as in the action of gastric juice on a nucleoprotein. By the action of caustic alkali the remaining protein in nuclein may be split off, leaving nucleic acid.

This nucleic acid is by far the more interesting part of the nucleoprotein molecule, and it has been much studied. By vigorous hydrolysis nucleic acid may be split into four kinds of material (1) purine bases, (2) pyrimidine bases, (3) a carbohydrate, and (4) phosphoric acid. Four purine bases are obtained, adenine, guanine, hypoxanthine and xanthine. Only the first two are considered to be present in the original molecule, the last two being formed from the others in the process of hydrolysis. In the body a fifth member of the group is formed from the others,-uric acid.<smiles>O=c1[nH]c(=O)c2[nH]c(=O)[nH]c2[nH]1</smiles>

The pyrimidine bases are somewhat closely allied to the purine bases. Uracil, thymine and cytosine are the three members of the group obtained from nucleic acid.

The carbohydrate portion of the nucleic acid is sometimes a pentose and sometimes a hexose or allied substance.

The fourth product is phosphoric acid, $\mathrm{H}_{3} \mathrm{PO}_{4}$.

The structure of the nucleic acids has been worked out, and the student is referred to larger works for further discussion of this subject. 
The nucleoproteins may be extracted from tissue, such as the pancreas, by boiling with water. On acidifying with acetic acid the nucleoprotein will precipitate. In this and other properties the nucleoproteins resemble the globulins and the phosphoproteins. From both of these groups they may be distinguished by their content of purine bases. The nucleoproteins contain about the same per cent of phosphorus as the phosphoproteins.

Lecithoproteins.-The lecithoproteins are compounds made up of protein combined with lecithin or some other phosphatid. Little definite information is available concerning these compounds. Some investigators put vitellin in this class instead of among the phosphoproteins, for vitellin can only be freed from the lecithin which occurs with it, by extraction with hot alcohol. It has been suggested also that the residue of the blood corpuscles after removing hemoglobin contains a lecithoprotein. Our information about the group is still in a fragmentary and unsatisfactory state.

\section{Derived Proteins}

The derived proteins are produced when proteins are acted on by various agents. The group is divided into primary and secondary protein derivatives. In making the former the proteins are not greatly changed in their properties. In making the secondary derivatives, extensive alteration or breaking down occurs. The primary derivatives are again divided into proteans, metaproteins, and coagulated proteins. The secondary derivatives are subdivided into proteoses, peptones, and peptids.

Primary Protein Derivatives.-Proteans.-Proteans probably may be formed from most of the simple proteins. Those formed from the globulins have been most studied. If edestin, the globulin from hemp seed, is dissolved in the smallest possible amount of hydrochloric acid, it may be precipitated by adding a small amount of sodium chloride. On adding stronger salt solution a portion of this precipitate will not dissolve. As often as the above process is repeated, a further portion of the protein becomes insoluble. The same results are obtained by the action 
of weak alkalies, or the initial action of enzymes on proteins. The resulting substances are called proteans, and are named from the material from which they are prepared,-thus edestan from edestin, myosan from myosin, etc. They differ very slightly from unchanged proteins, and undoubtedly only minor rearrangements in the protein molecule have occurred in their formation.

Metaproteins.-Metaproteins are formed from the proteins by the action of alkalies or acids, either dilute or concentrated. They are sometimes called albuminates. If a very dilute acid or alkali is allowed to act on a protein at body temperature for some time, perhaps a half hour, it is quite evident that some change has taken place in the protein for if the faintly acid solution of the substance is boiled, no coagulation takes place. Both acid and alkali metaprotein, formed by using dilute acid or alkali, will dissolve in a slight excess either of acid or of alkali. If the solution is made exactly neutral, however, the metaproteins will precipitate. If this precipitate is suspended in water and boiled, it is coagulated, and no longer can be dissolved by adding dilute alkali or acid. Dissolving alkali metaprotein in acid, or acid metaprotein in alkali does not change these substances into one another. Perhaps acid metaprotein may be changed into alkali metaprotein by longer action of the alkali, but the reverse process surely does not take place. Alkali metaprotein is formed more quickly than is acid metaprotein, and in the former process some nitrogen and sulphur are split off. Acid metaprotein is particularly interesting because it is formed as the first stage in the digestion of most proteins in the stomach. Gastric juice contains hydrochloric acid which forms acid metaprotein from the proteins of the food, thus bringing them into solution. The metaproteins behave in most respects like proteins, and apparently the protein molecule is not extensively altered when they are formed.

Coagulated Proteins.-Coagulated proteins are formed when acid solutions of most proteins are heated to high temperatures, when most proteins are allowed to stand under alcohol, or 
are acted on by certain enzymes, and perhaps by other means. They are characterized by their insolubility in the usual mild protein solvents, such as water, salt solutions, etc. Little is known of what takes place in the protein molecule in coagulation. Although coagulated proteins are fairly insoluble, still they will dissolve in strong acids or alkalies, or in dilute acids or alkalies on warming slightly for some time. In the latter case they are converted into metaproteins. In the process of cooking food, most of the proteins are coagulated, but they are converted into metaprotein by the acid of the gastric juice, and then digested. Some proteins are digested more easily after coagulation, others less so. Coagulated proteins give all color tests given by the original protein. The protein molecule evidently has not been greatly altered or broken down in their formation.

Secondary Protein Derivatives.-Proteoses and Peptones are formed from proteins by the action of strong acids or alkalies, or by the action of enzymes. The protein molecule is broken up into fragments of varying size and properties. From what is known of the size and complexity of the protein molecule it is easy to understand the great diversity in size, composition and properties of the products formed on splitting it into fragments. Much labor has been spent in the study of these compounds. As yet little is known of the individual substances. For convenience, they are divided according to the concentration of ammonium sulphate required to precipitate them. Those which precipitate on saturating with ammonium sulphate are called proteoses. Those which will not precipitate on saturating with ammonium sulphate are called peptones. Such a method of separation is unsatisfactory, but it serves its purpose until more is known of the constitution of the individual substances. It is now known that factors other than the size of the molecule affect the ease with which a substance may be precipitated with ammonium sulphate. Thus substances of the above nature which contain tyrosin, cystin and tryptophane are precipitated at lower concentrations of ammonium sulphate than are those even 
of larger molecular weight in which these amino acids are missing. A study of the proteoses and peptones has developed the important fact that the breaking down of the protein molecule is by no means a symmetrical process. In fact, it probably consists in breaking off fragments of the greatest diversity, including some of the amino acids themselves even in the early stages of the process. There appear to be certain combinations or groups,- "nuclei" we may call them, in the protein molecule which are quite resistent to hydrolyzing agents, and even after very vigorous hydrolysis such groups may remain intact. Although the proteoses and peptones are simpler in structure than the proteins, they still are quite complex substances and contain many amino acid molecules, many more than the polypeptid of 18 amino acids built up by Fischer.

By boiling with strong acids or alkalies, or by the action of certain enzymes, proteoses and peptones are split into amino acids as might be expected.

Neither proteoses nor peptones coagulate on boiling. Both groups give the biuret test. The color is redder than that given by proteins, however. They usually give the xanthoproteic test. Their response to the Millon's and Hopkins-Cole reactions depends on the presence of tyrosine and tryptophane in the particular proteose or peptone under investigation. Proteoses usually give these tests. Peptones seldom do. Concentrated nitric acid precipitates some proteoses. These are called primary proteoses. It does not precipitate "secondary proteoses" or peptones. Peptones will diffuse fairly well through an animal membrane.

Peptids are substances consisting of only a few amino acids united by the "peptid linking." These compounds have been obtained by hydrolyzing protein, and also have been built up artificially in the laboratory, as already has been indicated at an earlier point in the discussion of proteins.

The final products of hydrolysis, the amino acids are the ultimate building stones of which the proteins and their simpler derivatives are built up. We have seen that proteins are neces- 
sary in the food to maintain life, but after all it is really the amino acids which are needed, for in digestion the proteins are split up into amino acids, and these substances are supplied to the living cells of the tissues. Some of these amino acids are used to build up new cell proteins or repair old ones, whereas others are transformed into various compounds some of which undoubtedly have most vitally important rôles to play in controlling or instigating many of the vital processes. Others of the amino acids undoubtedly are simply broken down and burned in the body as fuel, just as are carbohydrates and fats generally speaking. Some of the important rôles of the amino acids will be considered later in connection with the study of metabolism. 


\section{CHAPTER VI}

\section{SOME FAMILIAR FOODSTUFFS-SOME IMPORTANT TISSUES}

\section{Some Important Foodstuffs}

The substances considered in the preceding chapters are for the most part foodstuffs. It will be worth while, however, to consider some of the familiar substances usually called "foods." Most of these materials consist of a mixture, often of several of the classes or individuals of the material bases. We may ask the question, "What is a food?" In a broader sense a food may be defined as any substance which is required by the body. This would include oxygen from the air, water, and inorganic salts, in addition to carbohydrates, fats, proteins, etc. But some substances which are excellent foods, are not indispensable to the body, for example sugar. The body tissues are constantly wearing out and requiring repair. Materials for such repair of worn out tissues, or for the building of new ones, as in growth, are obtained from foods. Also, the body is constantly doing work, either external work, or the work of the heart, the digestive organs, glands, etc. Work cannot be done without the expenditure of energy. This energy is obtained by oxidizing, "burning," material derived either directly or indirectly from the foods. Some substances can be burned in the body, but still are harmful because of a poisonous effect upon the cells; such substances should not be classed as foods. Summing up the foregoing statements, a food may be defined as a substance which is necessary to the body or which furnishes it with energy or building material, and which is not harmful to the organism.

Water and salts, which are very important foods have been 
considered in connection with the general composition of the body.

- Cooking and Preparation of Foods.-Some foods are palatable and digestible when raw, such as milk, fruits, nuts and some vegetables. Other foods usually are prepared for use by cooking. Proper cooking of food is very important, both from an economic and from a physiological standpoint. Inexpensive materials, if properly prepared may be quite as good and desirable foods as more expensive materials, but even the best of materials may be spoiled and rendered unfit for use by careless or improper cooking. In cooking, various changes are brought about in the foods. Most animal proteins are coagulated, but this does not diminish, and sometimes increases their digestibility. The closely packed fibers of meat are loosened up so that they are more easily torn apart and subjected to the action of the digestive enzymes. Vegetable materials also are softened and made more vulnerable to the attack of enzymes. The taste and appearance of food materials also are improved in the process, and this appeal to the appetite is of actual physiological value in digestion. A further service performed by proper cooking is the destruction of living parasites of various sorts which often are found in raw foodstuffs, especially if the materials have been exposed in provision shops, or have been kept longer than is wise. Great progress has been made in recent years in regulating the care and quality of foodstuffs and thus in protecting the general public from dangers arising from the practices of ignorant, eareless, or unscrupulous dealers. Too much care and thoughtfulness can scarcely be spent in the proper choice and preparation of the diet.

Milk.-Milk is one of the most important natural foodstuffs. It is the natural and ideal food for the growing young, and comes very near to being an ideal food even for the adult. It contains all the necessary food substances, and in very nearly the proper proportions. If bread or crackers are eaten with it, it is an ideal food. The carbohydrate of milk, lactose, already has been discussed. Cow's milk contains about $4 \%$ lactose, hu- 
man milk about 6-7\%. Milk fat, about $2-4 \%$ in both cow's and human milk is characterized by its content (about $8 \%$ of the total fats) of lower fatty acids, butyric, caproic, caprylic and capric. There. also are traces of phosphatids. There are at least three proteins, $(0.7-1.5 \%$ in human milk and $2.5-4 \%$ in cow's milk). Casein makes up about $80 \%$ of the total protein in cow's milk, the remainder is mostly lactalbumin, with a small amount of lactoglobulin. Milk also contains a variety of salts including those of calcium, magnesium, sodium, potassium and iron; it contains phosphates and chlorides. Milk contains about $87 \%$ water.

"It has recently been shown that milk is a valuable source of vitamines. This will be discussed in the section dealing with these substances.

If milk is allowed to stand, bacteria decompose lactose forming organic acids. The milk becomes disagreeable in taste, and if the process goes far enough the casein is precipitated, and the milk is said to be sour. By properly sterilizing or pasteurizing milk, the bacteria are destroyed, and if it then is kept in closed vessels and not exposed to the air it will keep for a much longer time than otherwise. Half emptied milk bottles should on no account be left uncovered in a room or in the family ice chest, as bacteria from the air, falling into the milk, will thrive and multiply, and the milk if used may thus become a menace to health.

When milk stands for some time a portion of the fat rises to the surface. If this layer is skimmed off the liquid is called cream. Average cream contains $18-20 \%$ fat.

Buttermilk differs from sweet milk chiefly in its lower fat content (about $0.5 \%$ fat), since most of the fat has been removed as butter. As butter is usually made from old cream, buttermilk often is sour, that is, contains some lactic acid produced by the fermentation of lactose.

Butter.-Butter is a food of very high fuel value, for it contains $80 \%$ or more of fats. The composition of this fat already has been discussed (see under fats). Butter contains 
about $1 \%$ of protein, on an average of $3 \%$ salts and from 10 $15 \%$ water. It contains practically no carbohydrate. Butter is an excellent source of vitamine. Oleomargarine, a butter substitute made largely from beef fat also has been discussed in the chapter on fats.

Cheese.-When the caseinogen of milk is clotted by rennin it separates out as a solid curd, which carries .with it much of the milk fat. This curd when filtered off is called "cream cheese." From it, by pressing and other processes cheeses of various kinds are made. Cheese is a very nutritious foodstuff. Different kinds of cheese vary greatly in composition and food value. Average Amcrican cheese contains about $29 \%$ protein, $36 \%$ fat, traces of carbohydrate, $3.5 \%$ ash and $32 \%$ water.

Meats.-Meats are important food substances, for they furnish large amounts of proteins, which are essential constituents of the diet. Meats contain a variety of protcins, but the chief protein of dead muscle is myosin. Albumin, globulins other than myosin, albuminoids, glycoproteins, nucleoproteins, hemoglobin derivatives, and lecithoproteins all are constituents of meat, but in digestion all of these substances are broken up into their constituent amino acids, the form in which protein food reaches the cells of the tissues. Meat also contains fats, and small amounts of the carbohydrate glycogen or the sugar derived from it. Most meats, with the exception of bacon or very fat meats contain from 10 to $20 \%$ protein. Fat ranges from a few per cent up to $60 \%$ in fat bacon. Ash ranges from about $1 \%$ to 4 or $5 \%$, water from about 7 or $8 \%$ to 68 or $70 \%$.

Eggs.-Eggs are a very important article of diet. In the development of the young of birds they furnish the material out of which the tissues of the chick are built up. Eggs contain (without the shell) about $12 \%$ protein, $11 \%$ fat, $0.5 \%$ carbohydrate, $1 \%$ ash, and $75 \%$ water. Most of the fat is in the yolk. About $2 / 5$ of the protein is in the white and $3 / 5$ in the yolk. About $90 \%$ of the total protein of the white is albumin. About $61 / 2 \%$ is globulin. Vitellin is the chief yolk protein. All of these substances have been considered under 
their respective groups. The yolk contains fat and also considerable quantities of lecithin and cholesterol.

Vegetables.-Vegetables contain very high amounts of water and thus are low in fuel value. Some vegetables such as peas, beans, and lentils contain only small amounts of water. With the exception of these and a few others, most vegetables contain but small amounts of proteins, very little fat and varying amounts of carbohydrates. As fuel substances therefore most vegetables are far below meats and dairy products. Peas, beans and lentils are exceptions to this statement. Vegetables supply the body with some valuable salts, with some protein, fat and carbohydrate, but they are valuable also from another standpoint. They contain cellulose. Although cellulose is of little food value, it gives bulk to the food, and mechanically irritates the walls of the intestines. This stimulates muscular contractions which are important for the thorough mixing of the food with the digestive juices, and its transportation along the alimentary canal. The green leafy vegetables such as lettuce, cabbage, spinach, etc., are extremely valuable sources of vitamines, as will be pointed out later in the discussion of these substances. Fruits also for the most part have little food value, but they are wholesome for the same reason, and they also stimulate the appetite, which is an important factor in proper digestion. Nuts contain much protein, much fat and often considerable carbohydrate (peanuts contain protein 25\%, fat $38 \%$, carbohydrate $24 \%$ ) and thus are extremely nutritious.

Breadstuffs vary greatly in food value. Bread contains about $9 \%$ protein, very little fat, $53 \%$ carbohydrate and $35 \%$ water. Its fuel value is about that of average meats, but its protein content is lower. Crackers contain only about $5 \%$ water and their fuel value is about double that of bread. Sweet eakes, pastries, etc., usually have a high fuel value. They often are sufficiently indigestible, however, more than to counterbalance their greater fuel content. Some of the breakfast foods are valuable foods but many of them are so light that the amount ordinarily eaten has little total fuel value aside from that of the 
sugar and cream which usually is eaten with them, and which may be considerable.

Choice of Diet.-Much has been written upon the proper choice of a diet. Undoubtedly a mixed diet of wholesome nourishing food in sufficient quantity, but not in extravagant excess is the most desirable solution of the problem for the average healthy individual. Different standards of amount will be needed by individuals of different size or different habits of life. For an average sized (70 kilos) city dweller, perhaps 90 grams protein and enough fats and carbohydrates to make the total energy intake about 2,500 calories is about the right amount, but these figures will vary greatly with changing conditions. This subject is discussed in somewhat greater detail in the chapter on metabolism. The diet must contain certain unknown substances called vitamines, which will be discussed in the chapter on metabolism.

\section{Some Important Tissues}

Although the substances occurring in the different tissues have been discussed under the various groups of which they are members, it may be useful from the student's point of view to include here a brief summary or survey of some of the important tissues, and to discuss some additional points of interest.

Muscle.-The muscles make up about $2 / 5$. of the body weight in adults. They contain about $18-20 \%$ protein, $72-78 \%$ water and from $0.15-0.3 \%$ of glycogen. If muscle tissue is subjected to a very high pressure, a liquid known as the plasma is squeezed out. This represents about $60 \%$ of the total muscle weight. The remaining material is called the stroma. The plasma has the power of clotting. The chief proteins of the plasma are myosin and myosinogen (or myogen). Until recently most authors have considered myosinogen to be the mother substance of myosin. This has been disputed, however; it is considered by some investigators to be an albumin. The stroma contains a protein resembling an albuminoid. In addition to the above 
substances, muscle contains various extractives and salts, such as creatine, urea, inosite, taurine, and also some lipins. After activity, particularly if the muscle supply of blood or oxygen is low, lactic acid is found. This is believed to be a product of the partial oxidation of glucose. The lactic acid may be further oxidized, or rebuilt into something else.

The important property of muscles is their power of contracting. The process has been extensively studied, but as yet it is but imperfectly understood. Heat is liberated, glucose is used up and $\mathrm{CO}_{2}$ is given off. Possibly the swelling and shortening of the muscle fibers is caused by the acid and heat. The individual muscle fibers become shorter and thicker. The acid is quickly destroyed, $\mathrm{CO}_{2}$ is given off, and relaxation follows. If the acid is not removed, cramps or rigor result. It has been suggested that the swelling of the fibers is caused by taking up water under the influence of acid, since proteins swell in acid solution. The question is still unsettled, however.

A great deal of interest has centered around the chemistry of muscle activity, and the factors involved in the control of the blood supply to the muscles. Studies of the respiratory exchange, the composition of the blood and the changes taking place in excised muscles under various conditions all have contributed to our information, but many of the problems connected with muscle contraction still are unsolved.

During activity the blood supply to the muscles is greatly increased. If the activity is general, this is brought about by increased heart action and by dilation of the blood vessels in the muscles themselves. If the activity is local, the increase in blood supply is brought about mainly in the second manner. Even during rest a state of tonus exists, and $\mathrm{CO}_{2}$ is produced and $\mathrm{O}_{2}$ consumed. Indeed this is the case even if the nerve supply is cut so that the muscle loses its tone. In this case the extent of oxidation is greatly decreased. During contraction, the amounts of $\mathrm{CO}_{2}$ and $\mathrm{O}_{2}$ are very greatly increased. The nerve centers are stimulated, though it is not known exactly how. - An increase in the free $\mathrm{CO}_{2}$ in the blood, and a slight 
change in the reaction $(\mathrm{pH})$ of the blood have been suggested. In vigorous activity adrenalin secretion from the adrenal glands is augmented, resulting in increased vasomotor tone, and breaking down of liver glycogen (see section on metabolism of carbohydrates).

The production of acid also has an effect on the muscles themselves,- a dilation of the arterioles, and changes in the tone of the capillaries. If the supply of $\mathrm{O}_{2}$ is adequate, lactic acid either is not produced, or disappears very quickly. The earlier view that the disappearance of lactic acid in the period of relaxation was due to its oxidation has now been questioned. An excised muscle which of course is not receiving any repair material may be made to contract many times, and as much lactic acid can be obtained from it as could have been extracted after a few contractions. Also the oxidation of 1 gram lactic acid yields 3.7 calories, whereas 4.5 calories are produced in a muscle while 1 gram of lactic acid disappears. The belief is gaining ground that lactic acid goes back into its original form, while something else, perhaps glucose, is oxidized.

If a muscle is tetanized, the amount of lactic acid increases, and the muscle does not respond so well to the stimuli. If the circulation is vigorous, however, the muscle regains its activity. Fatigue is thus due to the lactic acid and perhaps other substances which accumulate in the cells during contraction. In smooth muscle contraction little energy is expended, hence smaller amounts of waste products are formed, and the smooth muscles are not susceptible to fatigue.

Whereas muscle contraction is accompanied by the appearance of lactic acid, it is interesting that the greater part of the oxygen consumed is used up after the period of contraction. Also the heat production lasts much longer than the contraction, so it appears that the contraction is not directly caused by the oxidation process.

During muscular activity, the amount of muscle glycogen is greatly reduced. It is generally considered that it is converted into glucose, which is oxidized with the liberation of energy. 
There is good evidence for believing that the cells can burm other things, however, for example, fats.

Practically nothing is known as to the actual course of the oxidation of glucose in muscle tissue.

Brain and Nerves.-Brain and nerve substance makes up a far smaller proportion of body weight than do the muscles, but the primary importance of this kind of tissue is obvious. The brain and nerves are the directing mechanism which controls the body activities. In higher animals, particularly in man where intelligence is at the maximum, the brain is relatively larger than in lower forms. The composition of brain and nerve tissue is thus of great interest. This kind of tissue contains less protein than the muscles. Gray matter contains $85+\%$ water, white matter $70+\%$ water. The characteristic fact in the composition of brain and nerve tissue is the presence of large quantities of alcohol-ether soluble substances. These include cholesterol, phospholipins, cerebrosides, but almost no neutral fat, possibly none at all. The principal phospholipins are lecithin, kephalin and various myelins; of the cerebrosides the most important are cerebron, phrenosin and kerasin. Various extractives also are found, such as creatine, inosite, purine bases, lactic acid and other substances. Caprine ( $\propto \mathrm{NH}_{2}$ caproic), an amino acid occurring in brain protein has been found in no other tissue. Glycogen is not stored in the brain.

The brain and nerves show a vigorous oxidative metabolism, producing $\mathrm{CO}_{2}$. They are very sensitive to lack of oxygen, and quickly cease functioning properly if their oxygen supply is eut off.

Nothing is known of the chemical changes involved in mental activity. Since the lipins are present in such large amount, it is possible that they are concerned in some way in these processes.

Bones and Teeth.-The bones and teeth, which form the solid structural portions of the body are composed of both organic and inorganic material. The bones are living tissue, and their cells wear out and are repaired just as any other cells. 
About $60 \%$ of bone tissue is organic material, largely the protein collagen. There also is some mucoid,-osseomucoid. In the hollow bones marrow is found, and this material contains much fat. There are two kinds of marrow, yellow and red, the red containing many red corpuscles. In the bones extensive amounts of inorganic material are found, chiefly calcium phosphate and carbonate.

The teeth are composed of three materials,-enamel, dentine, and cement. The enamel is the hardest substance in the body and contains only about .5\% of water and about $91 \%$ of calcium phosphate. Dentine and cement are of about the same composition as bone.

Dentine contains $26-28 \%$ of organic material. This yields gelatine on boiling, but it is considered to be not identical with collagen. Cement is practically identical with bone in composition. Bone ash has approximately the following composition: Calcium phosphate $87 \%$, calcium carbonate $10 \%$, calcium chloride $0.2 \%$, calcium fluoride $0.35 \%$, magnesium phosphate $1.6 \%$, iron oxide $0.1 \%$.

Gabriel has reported that the amount of fluorine in teeth is no greater than in the bones,-about $0.1 \%$.

Gassman reports that different teeth in the mouth of the same individual have different compositions, and he considers the frequent early decay of wisdom teeth to be due to a lower content of organic material and a higher content of lime than the other teeth.

Much work is needed to clear up the causes of dental caries. Rose considered tooth decay to be connected with a deficient supply of calcium salts. Recent work has brought out the apparent connection between one of the vitamines, $\mathrm{C}$, and the teeth. Degenerative changes in the teeth have been reported as the first effect of a shortage of $\mathrm{C}$ in the diet.

Cartilage contains collagen, chondroalbuminoid, salts and mucoid.

Connective Tissue.-There are two chief types of connective tissue, yellow and white. The white elastic tissue consists 
mainly of collagen, the yellow of elastin. Both forms contain mucoid and extractives.

The Blood.-Many points in connection with the blood have been considered elsewhere, chiefly in the discussion of hemoglobin in the chapter on proteins. Some additional points will be considered here. The blood is a fluid in which a variety of formed elements, corpuscles and platelets, are suspended. It is of the utmost importance as a circulating medium for it carries oxygen, $\mathrm{CO}_{2}$, food materials, products of internal secretion, various waste substances, heat, salts, etc., to or from the cells. The blood is thus the common carrier of the body, delivering fuel and other supplies to the cells, and carrying away the cell refuse. Each cell is bathed in an aqueous fluid just as were the original independent unicellular organisms which lived in the sea.

In recent years, much value has been attached to variations in the amounts of the nonprotein constituents of the blood such as urea, uric acid, creatinin, sugar, cholesterol, etc., in the diagnosis of disease. In the laboratory section will be found methods for the estimation of these various substances. In diabetes, blood sugar is high, in nephritis the amount of uric acid first increases, later that of urea, and still later that of creatinin. The following table has been compiled from the work of various investigators in this field,-Hammett, MacLean and others. Amounts are given in milligrams per 100 c.c. of blood. Insignificant decimals have been omitted.

Nonprotein constituents of blood in milligrams per 100 c.c. blood: (Normal)

$\begin{array}{ccccc}\text { Urea } & \text { Uric } & \text { Total non- } & \text { Sugar } & \text { Creatinine } \\ \mathrm{N} & \text { acid } & \text { prot. } \mathrm{N} & & \\ 10-25 & 0.5-1.16 & 27-45 & 85-166 & 0.37-0.6\end{array}$

Cholesterol

$140-180$
Chlorides

524-584

The general composition of the blood is as follows (from Mathews) : 


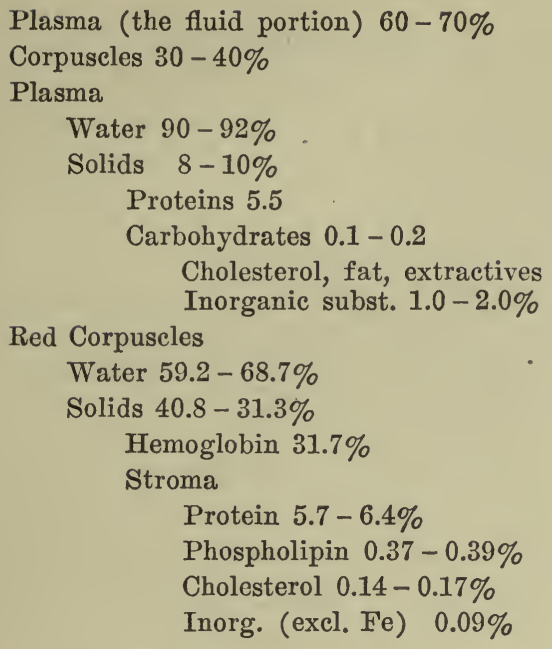

The important constituent of the red corpuscles is hemoglobin. This substance already has been discussed in the chapter on proteins (q.v.). It carries oxygen to the cells, and assists in carrying away $\mathrm{CO}_{2}$. In the transportation of $\mathrm{CO}_{2}$, other factors are important, for this substance is carried to a considerable extent also in the plasma, as sodium bicarbonate, carbonate and combined with plasma proteins. When $\mathrm{CO}_{2}$ passes from the tissues into the blood it makes the blood less alkaline. This favors the liberation of $\mathrm{O}_{2}$ from oxyhemoglobin. The reverse case occurs in the lungs.

The transference of $\mathrm{O}_{2}$ into, and of $\mathrm{CO}_{2}$ out of the blood during its passage through the lungs has been much studied to determine whether it is a process of simple diffusion, or dependent upon secretory activities of the cells of the alveolar epithelium. It is now believed that under ordinary conditions diffusion is the principal, if not the sole cause of the passage of gases to and from the blood. It is probable, however that in times of stress there may be an active secretion of gases by the cells to supplement the ordinary diffusion.

The white corpuscles are true cells, and possess a nucleus. 
They can move about, can reproduce, and have other functions characteristic of living cells generally.

The red corpuscles are living but contain no nuclei in mammals. There are from 5-6 millions per cubic millimeter in normal blood, but they may fall to one-half that number in certain diseased conditions.

The platelets are structures derived probably from the white corpuscles, possibly also from the red. They play an important rôle in the clotting of the blood. It has been reported that they contain nuclein, but this has been contradicted.

The chief plasma proteins are serum albumin, one or more serum globulins and fibrinogen.

The blood contains various enzymes in small amounts, e.g. amylase (acts on glycogen), a glycolytic enzyme (acts on glucose), lipase, proteolytic enzymes and an invertase, the amount of which is increased if cane sugar is injected or fed in large amounts.

A reaction which has attracted much attention in the last few years is known as the Abderhalden reaction. This investigator found that if a foreign protein is injected into the blood, enzymes appear which have the power of breaking down the albumoses produced by digesting that protein with cold $\mathrm{H}_{2} \mathrm{SO}_{4}$ for 24 hours. The serum of a pregnant woman will digest an albumose-peptone mixture obtained from placental tissue in this way. The digestion of the material can be followed by observing the slight change in optical activity of the mixture, or by dialyzing off the amino acids produced and confirming their presence by the ninhydrin test. There is much difference of opinion as to the value and reliability of this test for the diagnosis of pregnancy.

Reaction of the Blood.-The blood is almost neutral-it is alkaline to litmus and acid to phenolphthalein. Nevertheless, much acid must be added to blood before any great change in its reaction is produced. This is due to the fact that it contains $\mathrm{NaHCO}_{3}, \mathrm{Na}_{2} \mathrm{CO}_{3}$ and $\mathrm{Na}_{2} \mathrm{HPO}_{4}$, and alkali salts of the blood proteins. Any strong acid will form its sodium salts, taking the 
sodium away from the weak acid radicles enumerated above. These weak acids do not ionize to any great extent, and the reaction of the liquid remains near the neutral point. Acids produced in pathological conditions may be neutralized in a similar way.

The hydrogen-ion concentration of blood as determined by the gas chain or indicator method (see the discussion of $\mathrm{HCl}$ in the chapter on gastric digestion) is about $\mathrm{N} \times 10^{-7.2}$ to $\mathrm{N} \times 10^{-7.7}$ in defibrinated blood at $18^{\circ}-20^{\circ}$.

Osmotic Pressure.-The osmotic pressure of the blood influences the passage of water to and from the cells. It is kept at a uniform level by the kidneys, which excrete excessive amounts of salts, urea, and sugar. These substances are the main factors in determining the osmotic pressure of the blood. The average depression of the freezing point in mammalian blood is $\Delta=-0.5^{\circ}$ to $-0.6^{\circ} \mathrm{C}$.

Coagulation of the Blood.-An enormous amount of labor has been expended to clear up the mechanism of blood clotting. The results still are far from conclusive. Clotting is a protective device to prevent excessive loss of blood after injury. If blood is drawn and allowed to stand, it sets to a jelly-like mass. On standing, a yellow liquid is pressed out. This is called the serum. It differs from plasma in that it does not contain fibrinogen, for in clotting, fibrinogen is converted into the insol-" uble fibrin. This fibrin forms a fine mesh work in which the corpuscles are entangled. Contact with foreign bodies or with the injured tissue hastens clotting. Blood platelets and calcium play a rôle in the clotting process. In the process of clotting a new substance, thrombin appears. A second new substance, serum fibrinogen also has been reported.

The following summary of the steps involved in clotting is perhaps the most satisfactory explanation thus far advanced. Since clotting does not occur normally in blood circulating in the blood vessels, some one or more of the substances involved in clotting must be absent from such blood, or be held in check or in an inactive form. This substance is considered to be 
thrombin, which is present in the blood as prothrombin. Possibly prothrombin is combined with a substance "antithrombin." When blood is shed, as in injury, the blood platelets yield a substance thrombokinase which combines with antithrombin, liberating the prothrombin. The prothrombin then reacts with calcium to form thrombin. Thrombin acts on fibrinogen, which is converted into the insoluble fibrin.

Various things delay or prevent clotting, such as substances which precipitate calcium, strong salt solutions, alkalies, cooling, and a substance contained in the head of leeches, also some snake venoms and other poisons.

Lymph.-The lymph is a fluid much resembling blood plasma in composition. It fills the tissue spaces in immediate contact with the cells, and serves as a medium through which the interchange of material between blood and cells takes place.

The Skin.-The skin, hair, nails, etc., are made up chiefly of the protein keratin, and contain also various salts. The skin also contains various pigments, some of those of dark color being classed as melanins. 


\section{CHAPTER VII}

\section{DIGESTION IN THE MOUTH}

General Purpose of Digestion.-The body is by no means a permanent structure consisting always of the same molecules of its constituents. It is a living, changing mass of material, fairly uniform in its percentage composition, to be sure, but constantly wearing out, and being rebuilt or repaired. The cells perform much work, - they build up substances for secretion, they produce heat by the oxidation of their own constituents or materials supplied to them, and they perform mechanical work. To do all these things the cells require fuel, and materials out of which to construct their products. Thus a living organism must take food. For purposes of producing heat or mechanical work the materials required are not restricted to particular chemical substances. It is enough that the materials supplied be such that they can enter the cells, and be "burned" by them. For purposes of repair or building specific products the situation is different, however. Unless the body can build up a constituent such as a given amino acid required for the manufacture of a necessary substance, that particular constituent must be supplied in the food. We are beginning to realize that definite building stones are demanded by the cells for the construction of their products. In some cases the body itself is able to manufacture the necessary building stones from other materials. In other cases it is not able to do so, and serious consequences result if the required building stone is not supplied in sufficient amounts. This is another argument in favor of a varied diet which will be likely to furnish all substances 'required.

The materials of our food correspond only in a rough way to the materials out of which our body is constructed. Many of 
the plant proteins, for example, are valuable foods, although they differ from our body proteins in the relative amounts and in the arrangement of the different amino acids they contain. If the varied substances in our food all could get into the blood stream, and into the cells, the problems of the cells would be greatly complicated, for they would be obliged to deal with a great variety of proteins, carbohydrates and fats. Nature has solved this problem by arranging for the breaking up of the various foodstuffs into their simple building stones in the digestive tract. It is in the form of these simple building stones, a limited uniform list of which is obtained in general from all the food substances, that the food materials reach the cells. An exception is the case of the fats, the building stones of which are rebuilt for the most part into neutral fat before entering the blood. The primary function of digestion thus is to break up the diverse constituents of the foods into a fairly uniform mixture of simple, diffusible compounds which enter the blood and are presented to the cells for their use. The chief factors in this breaking down process are the enzymes present in the digestive juices. The enzymes and the conditions affecting their activities already have been discussed.

Preparation of Food.-CHewing.-Proper cooking is of great importance in preparing food, as was pointed out in the chapter on foods. After proper cooking comes proper chewing. The food should be thoroughly chewed to break it up into small fragments so that the digestive enzymes may have better access to its constituents.

Saliva.-Secretion, Amount.-In the process of chewing, the food not only is broken up, but it becomes mixed with the first of the digestive juices, the saliva. The saliva is secreted by three sets of glands, the parotid, submaxillary and sublingual, and also by small glands in the mucous membrane. The materials secreted by the different glands vary, as do also the nature of the stimuli which will cause a secretion. The blood brings to the salivary glands a supply of raw materials which are used in the formation of saliva,-salts, water, the constit- 
uents of mucin, ete. When the cells are secreting, their blood supply is greatly increased. Granules appear in the cells, and these apparently are the mother substance of mucin. The protoplasm of the cells is in large part converted into saliva, and then renewed from repair materials selected from the blood. Each set of glands is controlled by nerves from the brain direct, and from the sympathetic system. The nerves from the brain dilate the arterioles, those from the sympathetic system constrict. When the former are stimulated (the chorda tympani of the sublingual and submaxillary, and the auriculotemporalis branch of the fifth nerve, with its fibers from the glossopharyngeal for the parotid) much saliva is secreted, and it is of a thinner, more watery character than the secretion resulting from stimulation of the sympathetic. It is as yet undecided whether the difference in character of the saliva under these two types of stimulation is due to different responses on the part of the same cells, response of different cells, the dilation or constriction of the blood vessels, or to other causes. A flow of saliva may be produced by mechanical irritation of the mouth, by reflex action upon stimulation of the end organs of taste or smell, by purely psychie influences, such as the thought or sight of food, or by a general reflex, as in nausea preeeding vomiting. Its flow may be inhibited by nervous stress or anxiety, a fact well known to amateur public speakers or by those who have experieneed dryness of the throat in sudden fright. Some drugs stimulate, others retard the flow of saliva. The composition of the secretion is influenced by the nature of the stimulus producing it.

If a dog is fed meat, the saliva secreted is thick and mucous (submaxillary secretion) but if dried pulverized meat is fed, the saliva is thin and watery (parotid secretion). Thus there appears to be a certain amount of adaptation of the character of the secretion to the type of stimulus, and to the function which the secretion will be called upon to perform. The thick mucous saliva serves as a lubricant for the meat, whereas the thin watery secretion moistens the dry meat powder. 
Chemical substances may cause profuse secretion, for example dilute acetic acid. Weak alkalies do not stimulate secretion.

Very interesting results have been obtained in studying the psychic secretion. If a fasting animal is shown tempting food, saliva flows copiously. If the animal is not allowed to eat the food, however, and it is shown to him repeatedly, the psylehic secretion no longer appears. Also, if an animal is first shown something he does not eare for, and which causes no secretion, and then given something he is fond of, and the whole process is repeated several times, finally saliva will be secreted at sight of the unappetizing food, the display of which always has been followed by the feeding of something he likes. Evidently he has learned to associate the unappetizing food with the appetizing, a secretion resulting.

The amount of saliva secreted in a day undoubtedly varies within wide limits. 1500 c.c a day has been suggested as an average amount. Many factors may greatly increase this amount such as smoking, eontinuous chewing, mereury poisoning, or various drugs, notably pilocarpine.

Composition of the Mixed Saliva.-The mixed saliva is a thin watery fluid (99.4\% water) containing various salts, including potassium sulphocyanate, and, as its most important constituents, mucin and an enzyme ptyalin. Other substances of varying nature are present in small amounts. The saliva usually is somewhat turbid, due to cells or other material, and on standing the turbidity increases, due to the precipitation of calcium carbonate. It often is thick and somewhat slimy in character from the mucin present, most of which is secreted by the submaxillary and sublingual glands. Mucin is a glycoprotein which already has been discussed in the chapter on proteins. Saliva is usually slightly alkaline in reaction, (H-ion concentration about $2 \times 10^{-8}$ ) but so slightly that it is acid to phenolphthalein. In certain individuals the saliva is somewhat acid. Some recent reports on the reaction of the saliva have been to the effect that it normally is slightly acid in character.

Functions of Saliva.--The saliva moistens the mouth and food, 
and by reason of the mucin present, serves as a lubricating agent to aid in the manipulation and swallowing of the food. It also cleans and preserves the teeth, both by washing away particles of food which otherwise might decay, producing acids which would attack the teeth, and also by neutralizing acids introduced into the mouth. The saliva also performs an important digestive function by reason of the enzyme ptyalin which it contains. Ptyalin is an enzyme which acts on starches. Little is known of its chemical nature, but evidence seems to indicate that it is made up of a substance resembling a protein combined with a carbohydrate gum. Perhaps ptyalin is not a single enzyme, but a mixture of two or more enzymes, each of which is responsible for one of the steps in the digestion of starch. Ptyalin is a member of the group of amylases, or starch splitting enzymes. These enzymes are widespread in nature, and are found in vegetable cells and also in various animal secretions and tissues. They convert starch or starch-like carbohydrates into intermediate decomposition products and finally into maltose. The different amylases are not identical, however, as they are destroyed at different temperatures, and show other variations in their properties. The composition of the amylases is of course unknown, but they have been much studied. They may be extracted from tissues by water and precipitated with magnesium sulphate, the method of Lintner. Ptyalin itself is very difficult to isolate and purify. Practically all amylase preparations contain phosphate. It is likely that the phosphate is essential to the action of the enzyme. As stated above, ptyalin appears to be combined with a carbohydrate gum, which may act as a carrier. Ptyalin is secreted by the parotid glands. It acts best at a temperature of $40^{\circ}-45^{\circ} \mathrm{C}$. and is destroyed by heating rapidly to $75^{\circ} \mathrm{C}$. Ptyalin acts best in a very faintly acid solution, the optimum acidity being $10^{-5.3} \times$ normal. If the acidity is sufficient to turn congo red to violet $\left(\mathrm{N} \times 10^{-4}\right)$ the action of ptyalin is inhibited. It also will act in a weakly alkaline solution such as the saliva. The presence of some salt favors its action. If the salt is removed by dialysis, the ptyalin 
activity is greatly decreased, and the addition of sodium chloride will restore it. Phosphates also have a favorable effect on some of the amylases. The same amylase, in the presence of chlorides, may'act best at a $\mathrm{pH}$ different from its optimum in the presence of phosphates. Some salts have a remarkable inhibiting effect on ptyalin action, particularly salts of the heavy metals. The speed of ptyalin action also is retarded by the end products produced. This effect cannot be due simply to the reversibility of the process, however, as sugars other than maltose also retard ptyalin action. The sugars are believed to combine with the ptyalin, thereby reducing its activity. The speed of digestion by ptyalin is proportional to the amount of the enzyme.

Starch is attacked by ptyalin and broken down into simpler substances. The physical condition of the starch is important, for raw starch is digested only with difficulty, whereas cooked starch is digested very rapidly and completely under favorable conditions of temperature, acidity, ete. Various products are produced in the breaking up of starch, first the dextrins, of which there undoubtedly are several, and finally maltose and isomaltose. The progress of the decomposition may be followed by testing small portions of the liquid with iodine. The blue color characteristic of starch gives place to a purplish, then a red color, and finally no color whatever is produced. The products corresponding to these stages are as follows:

Color with Iodine

Starch $\rightarrow$ Amylodextrin $\rightarrow$ Erythrodextrin $\rightarrow$ Achroo-dextrin

blue

blue

red

(no color)

$\rightarrow$ Maltose

(no color)

Maltose undoubtedly is split off even in the first stages of the process. It also is possible to follow the progress of the reaction by observing the time required for the clearing of an opalescent starch solution, or the change in the optical activity of the starch solution, or by the use of Mett's tubes filled with starch. 
The Mett method is described in the chapter on Gastric Digestion.

The saliva also contains small amounts of an enzyme maltase, which breaks up some of the maltose into glucose. Perhaps, as already stated, there are different enzymes which are responsible for the different steps of the decomposition of starch.

Since the action of ptyalin is inhibited by concentrations of acid such as are present in gastric juice, it might be expected that its activity would last only during the brief time before the food enters the stomach. It has been shown, however, that when a meal is taken, the food forms a bolus or mass in the stomach and does not become thoroughly mixed with the acid gastric juice for some time, perhaps a half hour. The ptyalin thus continues to act under these conditions for some time after the food is swallowed, and the decomposition of starchy materials may be considerable. Ptyalin is not found in the saliva of all animals. It is missing, for example, in the saliva of the dog, cat and some others of the carnivora. Their natural food is not starchy in character.

The saliva also contains an enzyme erepsin which has the power of splitting peptids, but saliva does not digest proteins or fats. Certain substances are excreted in the saliva. Potassium sulphocyanate is present in small traces, but is not known to perform any definite function. If a capsule containing potassium iodide is swallowed, iodide soon may be demonstrated in the saliva. It is taken out of the blood and excreted by the salivary glands. Urea, uric acid and many other substances also have been found in traces in the saliva. Perhaps they merely filter through from the blood. 


\section{CHAPTER VIII}

\section{DIGESTION IN THE STOMACH}

Importance.-When the food has been mixed with the saliva and ground into small pieces by the teeth it is swallowed, and passed down the esophagus into the stomach. The stomach is a muscular, strong walled organ, which is collapsed or contracted upon itself when empty, but is capable of being greatly distended. If water is taken alone, it quickly runs through the stomach into the intestine, but solid food collects in a more or less solid mass near the entrance. It remains in the stomach for a considerable time, (1-5 hours) passing on little by little as it is thoroughly mixed with the gastric juice. The stomach thus serves primarily as a reservoir into which considerable amounts of food may be taken at a time. Since its walls are strong and muscular they are in no danger of being ruptured. The stomach thus stands between the more delicately constructed intestine and the mass of food taken in a normal meal, and passes along the food material in small amounts so as not to overtax the capacity of the intestine. However, the stomach also fulfils important digestive functions.

Methods of Study.-The digestive functions of the stomach are due to constituents of a juice secreted by numerous small glands scattered in the stomach wall. The study of this juice and its activities has received the attention of scientific men for a very long time. One of the chief difficulties was the manner of obtaining juice for study. Spallanzani, working in the latter part of the eighteenth century, swallowed food tied up in small linen bags which ultimately were passed with the feces. By studying the residues in the bags, the effects of digestion on the food could be observed. Another method consisted in swallowing a sponge tied to a string. The sponge soaked up 
gastric juice, and was then pulled out. Such methods were obviously crude, and led to but imperfect results. At the present time, to study gastric contents a light meal is given, and after a time the stomach content is pumped out with a stomach pump. This material consists of gastric juice mixed with food, but the method may give data valuable for diagnosis.

The first successful study of gastric juice and the conditions causing its flow was made possible by an accident which occurred in 1822 on the Island of Mackinac. Alexis St. Martin, a Canadian coureur $d u$ bois, was accidentally shot in the abdomen. The bullet passed through the stomach, and when the wound healed, a passageway was left from the stomach to the exterior. St. Martin recovered and lived for a long time in perfect health. He came under the care of Dr. William Beaumont, an American army surgeon, who recognized the exceptional opportunity to study the problems of gastric digestion, and made a long series of careful investigations. Two Europeans developed the same method of study by producing artificial openings or "fistulæ" into the digestive tract of dogs. The Russian, Pavlov, has brought the technique of the method to its highest stage of perfection. An incision was made into the stomach, and the cut sewed up in such a way as to produce a small pouch, or "little stomach" separated from the main stomach cavity, but still supplied with blood vessels and nerves. From this pouch, a silver tube led to the exterior. If gastric juice was poured out into the small pouch, it could be drawn off to the exterior and its composition and properties studied. These observations made on dogs have been supplemented by occasional investigation on human beings when restriction of the esophagus or other causes made it necessary to make an opening into the stomach from the exterior. By the use of such methods a large number of investigators have studied the problems. of gastric secretion and digestion. The results of these studies are summarized in the following discussion.

Causation of Flow of Gastric Juice.-The interior of the stomach, when no food is present, is pale pink and velvety in 
appearance. Gastric juice does not flow continuously. There are two factors which cause a flow to begin, nerve impulses and chemical stimulation of the glands. The former may have two origins, psychic or reflex. The mere thought or sight of food may cause a flow of gastric juice. This is known as the psychic or appetite secretion. The presence of food in the mouth gives rise to a copious flow by reflex action. The flow produced by nervous stimulation is large in amount, and continues for some time. It does not, however, account for all of the gastric juice poured out, for if a dog is allowed to swallow the food which he chews, the amount of juice obtained is greater than when the food is caused to drop out of an opening made into the esophagus. The presence of the food in the stomach thus is responsible for a portion of the secretion. Some investigators, among them Beaumont, have reported that mere mechanical stimulation of the stomach walls by means of a feather or a glass rod would cause secretion. Pavlov believes, however, that mechanical stimulation causes no flow. The sccretion resulting from the presence of food in the stomach is not due to reflex nervous impulses acting on the gastric glands, for it occurs even after the nervous connections of the stomach have been severed. All foods will not cause a flow, therefore it cannot be the result of mechanical stimulation. It is evident that a chemical substance must be involved, which stimulates the gastric glands to action. Injecting a partially digested mixture into the blood does not cause a flow. From this and other evidence it has been concluded that by the action of the food on the pyloric mucous membrane a substance is produced which is given off to the blood stream. When this substance, to which the name of gastrin has been given, reaches the gastric glands, it incites them to secrete gastric juice which is poured out into the stomach cavity. This subject is further discussed in the chapter on Intestinal Digestion. Some foods, such as meat broth, produce this effect directly. Other foods do so only after they are partly digested. This chemical secretion, though smaller in amount than the nervous secretion, persists for a much 
longer time. Digestion in the stomach thus is started by a copious flow of juice incited by nervous impulses, either psychic or reflex. The process is continued by a flow which is incited by the presence of the food itself in the stomach by means of a chemical substance, gastrin, produced in the mucous membrane, given off into the blood, which upon reaching the gastric glands, incites them to secrete.

The amount of gastric juice secreted varies greatly. It is roughly proportional to the amount of food eaten. From 2-3 liters a day has been estimated as an average amount.

General Character of the Secretion.-The character and composition of the gastric juice vary with the nature and amount of food taken. Perhaps this is due to the fact that all of the glands producing gastric juice may not be secreting all the time. The juice first secreted has a greater digestive activity than that secreted later, and any sudden increase in the amount of flow also is accompanicd by increased digestive power. The digestive agents apparently accumulate in the gland cells during rest; the first secretion would thus be more active.

Gastric juice from dogs or man is a clear, watery fluid, and is colorless unless mixed with bile which sometimes gets into the stomach through the pylorus. The specific gravity ranges from 1.002-1.0059. It contains the salts which are found in the blood serum, and some organic material. Its most important constituents are hydrochloric acid and the enzymes pepsin, rennin, and a lipase. The chief digestive activity of the stomach is on proteins, since pepsin and rennin both act on that group.

Hydrochloric Acid.-The first stage of the digestion of proteins in the stomach consists in their conversion into acid metaprotein by the action of the hydrochloric acid present in the gastric juice. Variations in the amount of acid occur, and if its concentration departs sufficiently from the normal, grave digestive disturbances result. Variations in the amount of hydrochloric acid accompany certain pathological conditions. For these reasons, the acid of the gastric juice long has been the sub- 
ject of much study. The total acidity of the gastric contents is due to the sum of several factors,-- free hydrochloric acid, hydrochloric acid combined with protein, acid salts and small amounts of organic acids. It may be determined as follows. The patient, in the morning, when the stomach is empty, is required to eat a "test meal," which consists of a cup of tea and a piece of toast, or some other simple articles of diet. At the end of a specified time, for example forty-five minutes or one hour, the patient is made to vomit, or his stomach is pumped out with a stomach pump. A measured amount of the unfiltered stomach contents is titrated with $\mathrm{N} / 10 \mathrm{NaOH}$ using phenolphthalein as indicator. This indicator is sensitive to such small amounts of hydrogen-ions (thus to such weak acidity) that the method will yield results corresponding to the total acidity from all factors. 100 c.c. of normal gastric contents should require about 74 c.c. to 90 c.c. of $\mathrm{N} / 10 \mathrm{NaOH}$ to neutralize it. By the use of other indicators which are less sensitive to weak acids, it is possible to decide whether all of the acidity is due to free hydrochloric acid, or part of it to hydrochloric acid combined with protein or to weaker acids. Gunzberg's and 'Toepfer's reagents are the best indicators to determine free hydrochloric acid. It is obvious that a large amount of a weak acid will produce as high a concentration of hydrogen-ions as a small amount of a strong acid. For this reason the above methods, and other similar methods for determining the various factors making up total acidity are inaccurate. The accurate determination of hydrogen-ion concentration is made by the gas chain method, a process which is too difficult for the average physician. The following method gives approximately correct results. It consists in using Gunzberg's reagent, which contains $2 \mathrm{~g}$. phlorglucin, and $1 \mathrm{~g}$. vanillin to 100 c.c. alcohol. The reagent should be kept in the dark. A drop of the reagent on a white plate is dried on the water bath. A drop of the juice to be tested is added to the yellow spot. On warming, if free hydrochloric acid is present a red color develops. By diluting, a point will be found where the liquid just gives the reaction. At this point the concentration 
of $\mathrm{HCl}$ is $0.0004 \mathrm{~N}$. Weaker acid gives no color. The results of this test correspond fairly well with those obtained by more accurate methods. In pure gastric juice most of the hydrochloric acid is free. Total acidity as determined with phenolphthalein, and free hydrochloric acid as determined with Gunzberg's reagent agree closely. If food is present however, the free hydrochloric acid is greatly reduced since much of the acid is combined with protein.

Human gastric juice contains on an average $0.3 \% \mathrm{HCl}$. (That of dogs contains about $0.6 \% \mathrm{HCl}$ ). The concentration of $\mathrm{H}$-ions runs from $10^{-2}$ to $7 \times 10^{-2} \mathrm{~N}$.

In diseases the amount of hydrochloric acid may vary considerably. A condition in which the amount is abnormally high is spoken of as hyperacidity (or hyperchlorhydria). This is usually observed in cases of gastric ulcer and in neurosis. If the acidity is below normal it is spoken of as hypoacidity (or hypochlorhydria). This condition often is observed in cases of cancer of the stomach or other regions. Sometimes no acid at all is secreted. This condition is called anacidity (or achlorhydria). A normal condition is called euchlorhydria.

Source of the Hydrochloric Acid.-The hydrochloric acid is secreted mainly by glands in the fundus end of the stomach. It is not stored in the cells previous to secretion, and probably is not even secreted directly by the cells, but is formed from a secreted substance. The actual mechanism is a matter of doubt. Perhaps it is secreted as an ester, or is formed from ammonium chloride or some other chlorine compound. The source of the chlorine is evidently the sodium chloride of the blood.

The functions of the hydrochloric acid are various. First, as we already have seen, it dissolves the proteins of the food, converting them into acid metaprotein. It plays an important part in digestion of proteins by pepsin, however, for this enzyme is secreted in an inactive form, and becomes active only under the influence of the hydrochloric acid. An unimportant phase of the function of the hydrochloric acid is in connection with its action on saccharose. This disaccharide is easily hydro- 
lyzed by dilute hydrochloric acid, and it is probable that this process takes place at least to some extent in the stomach. The hydrochloric acid also kills bacteria and parasites in the food, and thus protects the body from their attack.

Enzymes of the Gastric Juice.-Three important enzymes are secreted in the gastric juice, pepsin, rennin and a lipase.

Pepsin is formed in glands of the stomach wall, particularly in the fundic region but it exists there probably in an inactive form called pepsinogen, which becomes active only after secretion. Pepsinogen is stored up during the period of inactivity between times of secretion. The transformation of pepsinogen into pepsin is brought about by the hydrochloric acid of the gastric juice. Little is known of the nature of pepsin. Probably it is similar in nature to the proteins, or perhaps only attached to a protein, but this is still uncertain.

Pepsin, which digests proteins, acts best in weak acid solution. For pepsin from man, the most favorable acidity is about $0.3 \%$ hydrochloric acid or a hydrogen-ion concentration of about $1.7 \times 10^{-2} \mathrm{~N}$. to $3 \times 10^{-2} \mathrm{~N}$. At $10^{-4} \mathrm{~N}$. digestion almost stops. The most favorable acidity for pepsin corresponds closely with the normal acidity of the gastric juice of the animal from which the pepsin is obtained. Other acids may be used in place of hydrochloric, but they are not so favorable. Pepsin is very sensitive even to slight amounts of alkali, and is rendered inactive if its solution is made alkaline. For the action of pepsin it is not necessary that the hydrochloric acid be free. If the acid is combined with the protein acted upon, a neutral pepsin solution will digest the protein quite readily. Hydrochloric acid alone will digest proteins, but much more slowly than pepsin and acid together.

There are various methods for estimating the activity of a pepsin solution. The use of Mett's tubes is one of the most satisfactory. Egg white is drawn up into narrow glass tubes and coagulated by heating. The tubes then are cut into short lengths (ca. $2 \mathrm{~cm}$.) and suspended in the enzyme solutions. These are placed in the incubator for 24 hours. The amount of diges- 
tion of egg white bears a relationship to the digestive power of the solution. The length of the column of egg white digested is proportional to the square root of the amount of the enzyme. The products produced by the digestion of the protein inhibit the digestive action of the pepsin, so the method is not an ideal one, but it is useful for obtaining comparative data.

Products of Peptic Digestion.-Pepsin-hydrochloric acid attacks the proteins and breaks them up into fragments. The acid metaprotein first breaks into proteoses of varying degrees of complexity. These are again broken into products which are not precipitated by saturation with ammonium sulphate,- the peptones. It is probable in fact that peptones also are produced directly from the protein molecule in the first stages of hydrolysis. There has been much discussion as to whether or not amino acids are split off by pepsin. Weight of evidence seems to indicate that pepsin does not split off amino acids, and that the presence of these substances in mixtures digested by an extract of the stomach wall was due to another enzyme, called erepsin, or to still other agencies. The proteoses and peptones produced by the action of pepsin vary greatly in their composition and properties, as might well be expected on considering the extremely complex nature of the protein from which they are produced.

The ultimate fate of pepsin is a matter of interest, of which little is known. It probably is largely destroyed in the intestine, but a portion undoubtedly is absorbed into the blood. What happens to this portion is not known, but a part at least is excreted in the urine. Perhaps the remainder is destroyed by agents in the blood.

Rennin.-Gastric juice, or the water extract of the stomach mucosa of a young animal contains an enzyme rennin, which is instrumental in clotting milk. For this clotting, calcium also is necessary. The process is believed to consist in the transformation of the soluble protein caseinogen into a derivative called paracasein. In this process an "albumose" or "whey protein" is split off. The calcium salt of caseinogen is soluble, but that of 
paracasein is insoluble. When rennin has transformed the caseinogen into paracasein, the calcium salt of the latter precipitates, and the milk is said to be clotted. Removal of the calcium by precipitation as oxalate will prevent clotting, but will not interfere with the formation of paracasein. When this has been formed by the action of rennin in oxalated milk, the rennin may be destroyed by boiling. Now, on adding a soluble calcium salt to the milk it will coagulate, showing that the transformation of caseinogen into paracasein has taken place in the absence of calcium.

Are pepsin and rennin identical? Not only does gastric juice clot milk, but extracts of a very large number of substances containing proteolytic enzymes show a similar behavior. The question has thus arisen, are pepsin and rennin identical, or are they different enzymes? This question is still in dispute. The trend of evidence seems to indicate, however, that they are not identical. Solutions have been prepared which showed-peptic but not rennin action, and also the reverse is true. Also, peptic digestion takes place only in acid solution, whereas rennin will act also in neutral or weakly alkaline solution. Pepsin itself undoubtedly can clot milk, however. There is still no uniformity of opinion as to whether the two enzymes are, or are not identical, although as already stated, opinion is tending toward the latter view.

The value of rennin to the young animal depends on the fact that by clotting, the chief protein of the milk is retained in the stomach instead of running on through into the small intestine. It is thus subjected to the action of the enzyme pepsin.

Gastric juice contains a lipase, which acts on fats. The action is not extensive however, except in the case of emulsified fats, such as those of milk, which are digested to a considerable extent.

The contents of the small intestine may occasionally regurgitate into the stomach. Ordinarily this takes place only to a slight extent. In this way the digestive enzymes of the intestine 
may get into the stomach and exert their characteristic activities there.

The stomach wall is not digested by the gastric juice because the hydrochloric acid cannot pass into the cells of the mucous membrane. These cells also undoubtedly contain antienzymes which counteract the effect of pepsin, and thus protect the tissues. After death however, or if the blood supply to a given area is shut off, the walls of the stomach are attacked and digested.

Passage of the Food Into the Intestine.-The food is carried along the stomach by waves of muscular contraction. The entrance from the stomach into the small intestine is guarded by a ring of contractile tissue which is closed ordinarily. When acid comes in contact with the stomach side of this ring, as it does when the food has been thoroughly mixed with gastric juice, the sphincter relaxes and allows a small portion of the stomach contents or "chyme" to pass into the small intestine. When this acid mixture comes in contact with the wall of the small intestine, the sphincter closes again. Thus the chyme is passed into the small intestine in small portions.

Gastric digestion does not complete the disintegration of the foodstuffs. It only starts the process, and prepares the food for further digestion by the enzymes in the intestine. In fact the stomach can be removed without causing death. In such case, only small amounts of food can be taken at a time, and its character must be carefully regulated. 


\section{CHAPTER IX}

\section{DIGESTION IN THE INTESTINE}

General.-Digestion in the mouth and stomach, although valuable in beginning the breaking down of the foodstuffs, does not fit the greater part of the food for utilization by the body. The final, and by far the most extensive portion of the task falls to the intestine.

When the acid chyme passes into the duodenum, or upper part of the small intestine it becomes mixed with alkaline digestive juices. Since pepsin is very sensitive to alkali, when this occurs the peptic digestion stops. We now know, however, that the contents of the small intestine may remain acid or neutral for some time. The digestive activity of pepsin may thus continue for a time after the chyme has left the stomach.

There are three important digestive juices secreted into the intestine, the pancreatic juice, the bile, and the succus entericus or intestinal juice. The methods employed in studying digestion in the intestine are in the main similar to those used for gastric digestion,--such as the preparation of fistulas or openings into the intestine, removal of duodenal contents by means of a tube passed down the esophagus and through the stomach, and various other devices.

\section{Pancreatic Juice}

General.-The pancreatic juice, or "external secretion" of the pancreas, is produced by this gland, which lies along the duodenum or close to it, and empties its secretion by two main ducts and sometimes also smaller ones. The opening of the pancreatic duct in man lies 9-10 $\mathrm{cm}$. below the pylorus. The amount of juice secreted varies with the nature of the food. It has been estimated to average 500-800 
c.c. per day. The secretion is continuous in herbivora, whose intestines normally are well filled. In man, however, the secretion is intermittent, and it is poured out in large amount when the acid chyme is passed into the duodenum from the stomach.

Mechanism of Flow.-The factors causing a flow of pancreatic juice have been much studied. It became evident that the action of the acid chyme on the walls of the duodenum causes a copious flow. After severing the nervous connections of the pancreas, the introduction of acid into the duodenum still causes a flow of juice. Apparently it is not caused by reflex nervous stimulation alone. It was thought that perhaps the stimulation is due to a local reflex, but Bayliss and Starling showed that an acid extract of the walls of the duodenum, if neutralized and injected into the blood stream causes the pancreas to secrete. Apparently then, the action of the acid chyme on the walls of the duodenum causes a substance to be given off into the blood stream. This substance, when it reaches the pancreas, causes it to secrete. The substance has been given the name "secretin." Substances of this nature undoubtedly are formed by many tissues or organs in the body and sent off by way of the blood as chemical messengers to arouse activity in some other organ or tissue. To these substances, as yet of unknown constitution, the name hormone (derived from a Greek word meaning "I arouse to activity") has been given. An example of such a substance already has been cited in connection with gastric secretion. The final proof of the existence of intestinal secretin was obtained by making a cross circulation between two dogs so that blood from one also circulated in the other. Acid was placed in the-duodenum of one dog, and the pancreases of both began to secrete, showing that the stimulating substance had been carried in the blood.

It is very probable that secretin is not responsible for the entire secretion of the pancreas, but that a reflex stimulation from the walls of the duodenum also comes into play.

Composition of Pancreatic Juice.-Human pancreatic juice is a water-clear liquid, alkaline in reaction since it contains sodium carbonate. It requires from $10-15$ c.c. $\mathrm{N} / 10 \mathrm{HCl}$ to neutralize 
100 c.c. of juice, using litmus as indicator. The concentration of hydroxyl-ions is about $.0001 \mathrm{~N}$. It contains protein in quantities sufficient to cause a turbidity on boiling, and several enzymes, chiefly trypsin, lipase, amylase, nuclease, maltase and, at least in young animals, a lactase.

Trypsin.-Trypsin is a proteolytic enzyme. When secreted by the gland it has very little digestive action, but if pancreatic juice is mixed with the secretion of the walls of the small intestine, it becomes very much more active, and digests proteins vigorously. This fact is generally believed to indicate that trypsin is secreted in an inactive form, called trypsinogen, which is converted into active trypsin by a substance, "enterokinase," in the intestinal secretion. The nature of the activation is not understood. Perhaps trypsin is liberated from some other substance with which it is combined. Some authors report also that the bile has a favorable action on tryptic digestion. It has been reported that some other substances have the power of activating trypsinogen.

There is some difference of opinion as to the most favorable reaction for tryptic digestion, which is usually stated to be a slightly alkaline reaction. It is certain, however, that trypsin will act in alkaline, neutral or even faintly acid solution. The reaction of the duodenal contents varies in fact, being at first acid, until it is neutralized or made alkaline by the various digestive juices poured into the intestine.

Activated trypsin attacks most proteins very vigorousiy, breaking them down into proteoses, peptones, peptids and even amino acids. The digestion is by no means complete, however, and there still are many of the partially digested products. The digestion is more vigorous and far reaching than that of pepsin, and differs. from it also in the fact that it will take place in alkaline solution. The previous action of pepsin on certain proteins appears to make them more vulnerable to the attack of trypsin.

The activity of a trypsin solution may be studied by methods 
similar to those used for studying peptic activity, e.g., Mett's method, ete.

Pancreatic juice also contains an enzyme called erepsin, which acts mainly on the simpler digestion products, proteoses, peptones and peptids, breaking them down into amino acids. Erepsin acts also on some proteins, such as casein. Thus the final stage in the intestinal digestion of proteins is brought about by the erepsin of the intestinal juice, or "succus entericus." An erepsin also is present in the intestinal mucosa and in fact in most tissues. Erepsin reduces most of the protein digestive products to the amino acid stage, the form in which they enter the blood.

It is interesting that the kind of amino acids present and their arrangement are important factors in determining whether or not a given polypeptid will be digested by erepsin.

Rennin.-Pancreatic juice has the power of clotting milk, a "rennin" action. This is thought by some authors to be due to the pancreatic erepsin.

Action on Fats.-Steapsin.-Pancreatic juice has the power of cmulsifying and splitting fats. If mixed with neutral olive oil, the mixture quickly becomes acid. An enzyme called "steapsin," a lipase, splits the neutral fat into glycerine and falty acids. Steapsin is believed to be produced by the cells of the pancreas. It is by far the most important of the fat digesting enzymes. It acts best in a weakly alkaline solution, the optimum hydrogen-ion concentration being $\mathrm{N} \times 10^{-8}$. In stronger alkali, or in weak acid solution its activity is greatly reduced. Bile has a very favorable effect upon the activity of steapsin. This is partly due to the fact that bile emulsifies the fats and thus makes them more accessible to the action of steapsin, but this does not entirely explain the favorable effect of bile. The bile salts evidently are the bile constituents concerned. The liberated fatty acids combine in part with the alkali present to form soaps.

Action on Starches.-Amylase or "Amylopsin." - The pancreatic juice contains an enzyme "amylopsin" which acts on starch and glycogen, splitting them into the dextrins and finally 
maltose and isomaltose in much the same way as the ptyalin of saliva splits starch. Starches which escape salivary digestion, or starch and glycogen eaten by carnivora whose saliva contains no ptyalin thus are digested in the intestine. It has been suggested that perhaps amylopsin is really a mixture of two or more enzymes, which are responsible for different phases of the splitting of starch into the disaccharide maltoses, one enzyme taking the material as far as the dextrin stage, the other converting dextrins into maltose and isomaltose. The bile seems to have little or no effect upon the activities of amylopsin. The final stage of the digestion of starch is due to an enzyme maltase which splits maltose into glucose. Pancreatic juice of young animals also contains a lactase which splits lactose. This disappears as the animal grows older unless milk still forms a part of the diet as in man, pigs, and some other animals. Pancreatic juice has been reported to contain a nuclease which acts on nucleic acid, and splits it into its component parts.

\section{The Bile}

Causes of Flow. Amount.-The bile, secreted by the liver into the gall bladder is poured out into the duodenum by way of the bile duct. It is produced continuously by the liver, but its flow from the gall bladder into the duodenum is intermittent. The mechanism by which the secretion of bile into the digestive tract is controlled is little understood. The action of the acid chyme upon the walls of the duodenum seems to be one of the factors concerned. Probably this is due to hormone action.

The amount of bile secreted by the liver of man in the course of a day has not been determined accurately under normal conditions. 550 c.c. per day has been suggested as a possible amount. It may be less than this, but in all probability the amount is greater. Observations on this point have been made in cases of fistulae, but since the bile under these conditions does not enter the intestine, its neutralizing effect on the acid chyme is lost; this would tend to increase the formation of secretin. But bile 
itself, when it enters the intestine is said to increase the bile secretion. The point is thus still uncertain.

Composition. Function.-Human bile is a clear, watery, yellow, brown or greenish liquid as it is poured into the gall bladder. Here, however, it becomes somewhat more viscous, due in part at least to the addition to it of mucinous material from the mucous membrane of the gall bladder and biliary passages. Bile has a bitter taste, is usually somewhat alkaline in reaction and contains a variety of substances, among them bile pigments, bile salts, cholesterol, a mucinous material, inorganic substances and many other things. Although the bile itself does not contain digestive enzymes, at least in any important amount, it is of the greatest importance in the processes of intestinal digestion. This is especially true in the case of fat digestion. The addition of bile to a mixture of olive oil and pancreatic juice increases the amount of oil digested several fold. (5-10 times). There has been much discussion as to the constituent of bile responsible for this effect. Latest opinions ascribe it to the action of the bile salts, sodium glycocholate and taurocholate. There also are conflicting reports as to the effect of bile on the action of trypsin and amylase, but probably the former is not affected, and the latter slightly if at all. The function of the bile in connection with absorption will be discussed later.

Bile tends to reduce putrefaction in the intestine. It is not itself bacteriocidal, and probably this effect is due to its power of increasing digestion and stimulating the muscular movements of the alimentary canal, thus hurrying the food in its passage through this region.

Bile serves as an excretory medium for certain substances, among them cholesterol, the bile pigments and other materials.

Bile Pigments.-The bile contains a variety of colored compounds, the bile pigments. Bilirubin, a reddish brown pigment is probably the mother substance of most of the others. On oxidation it yieläs biliverdin (green), bilicyanin, and other compounds. On reduction it yields urobilin, one of the urinary pigments. The bile pigments are formed by the breaking down 
of hemoglobin of the blood. In their constitution they closely resemble other known derivatives of hemoglobin. They contain no iron. There has been much discussion as to where the transformation of hemoglobin into bile pigments takes place. It is probably in the phagocytic cells of the liver, which are believed to engulf the red corpuscles and destroy them. The liver cells then finish the transformation.

Bile Salts.-The bile salts, which are largely responsible for the favorable action of bile in digestion and absorption of fats, are mainly salts of glycocholic and taurocholic acids, two conjugated acids made up of cholic acid and glycocoll or taurine respectively. Cholic acid is a complex substance of which the formula is still uncertain. It is a specific product of liver cells and is formed nowhere else in the body. Bile salts if mixed with a little sugar and brought into contact with concentrated sulphuric acid give a violet color. This is known as Pettenkofer's test for bile salts.

Bile contains small amounts of sodium soaps of various acids. Bile from the bladder also contains a mucinous substance which is perhaps largely a phosphoprotein. The nature of this substance is not definitely known.

At times concretions known as "gall stones" form in the gall bladder. They are composed of cholesterol, inorganic material or bile pigment deposited from the bile.

\section{Intestinal Secretion}

The third of the important digestive fluids poured into the intestine is called the intestinal juice or succus entericus. The juice secreted into the duodenum is poured out by small glands in the mucous membrane, the glands of Brunner. Little is known of the mechanism by which these glands are made to secrete. They are stimulated to activity when the acid chyme passes the pylorus. The amount of juice secreted has not been definitely determined, but it probably is large. The juice is strongly alkaline, due to the presence of carbonates, and 
thus has an important part to play in neutralizing the acid chyme. This is by no means the only role of the intestinal juice, however, for it contains substances of the greatest importance in intestinal digestion. It contains enterokinase which greatly increases the action of pancreatic juice on protein. This is usually believed to be due to activation of the inactive trypsinogen, by conversion into trypsin. Enterokinase may be extracted from the intestinal mucosa from almost the entire length of the intestine to the rectum, but it is present in largest amount in the upper regions of the small intestine. Enterokinase is itself probably an enzyme.

Erepsin.-A second enzyme found in the intestinal secretion is erepsin. This enzyme is important, for it is responsible for the last stage in the digestion of proteins. Erepsin does not act on proteins themselves, with one or two exceptions, e.g., casein, but on the intermediate digestion products, proteoses, peptones and polypeptids, which it reduces to amino acids, the final digestion products of the proteins. Erepsin is found in the mucous membrane of the intestinal wall, and in fact, in most of the body tissues. Erepsin acts best in weak alkaline solution.

Other Enzymes.-Three enzymes which have the power of splitting disaccharides also are present in the intestinal juice, maltase, which splits maltose; lactase, which splits lactose, and is found in the intestinal juice of young mammals and in adults of animals which take milk in their diet, and invertase, which splits cane sugar.

The intestinal juice also contains a nuclease, which splits the nucleic acids of the nucleoproteins.

Excretory Function of Intestinal Secretion.-Aside from the digestive enzymes, the intestinal juice carries various substances into the intestine which are thrown out of the blood as undesirable, or waste materials. It thus serves in the capacity of an excretion as well as a secretion.

From the walls of the large intestine a secretion is poured into the intestinal cavity. This contains mucus as its chief con- 
stituent, and appears to contain no enzymes of digestive importance.

Bacterial Action in the Intestine.-The intestine shelters and favors the growth of enormous numbers of bacteria, which feed upon the substances of the intestinal contents. From these materials, bacteria produce a large number of compounds, some of which are harmless, but some extremely toxic to the body. The toxic materials are produced mainly from the digestion products of the proteins, the amino acids. By splitting out $\mathrm{COO}$ from the carboxyl group of an amino acid an amine is produced. These substances are known as ptomaines; many are extremely toxic. An example of this process is the formation of ethyl amine from alanine.

\section{$\mathrm{CH}_{3} \mathrm{CHNH}_{2} \mathrm{COOH} \rightarrow \mathrm{CO}_{2}+\mathrm{CH}_{3} \mathrm{CH}_{2} \mathrm{NH}_{2}$}

From tyrosine, by splitting off the side chain, a process which goes in several stages, phenol is produced

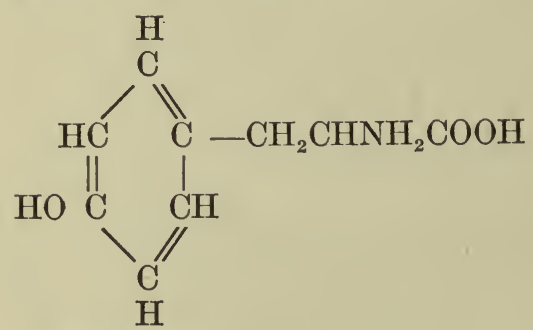

Tyrosine

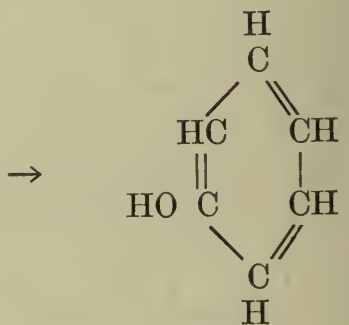

Phenol

From tryptophane indol and skatol are produced.

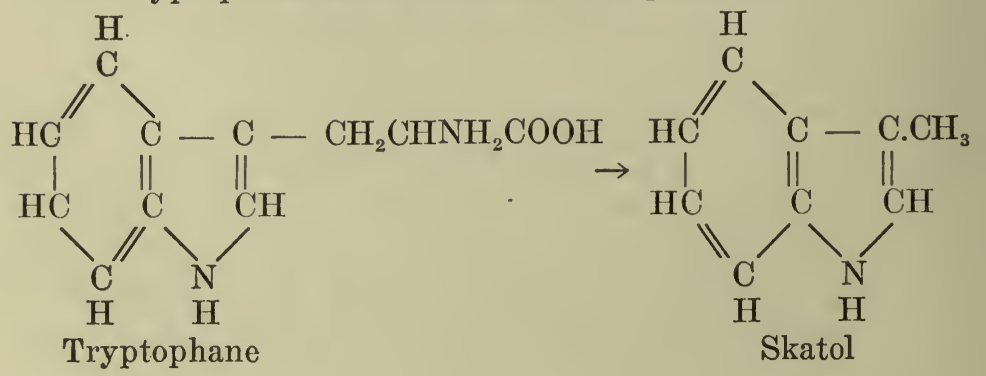




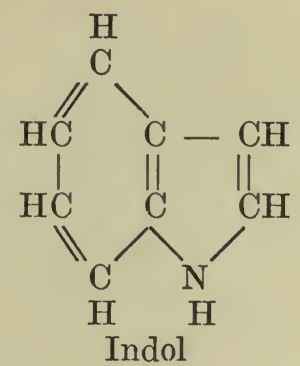

From cystin and cystein, hydrogen sulphide and mercaptans are produced.

Many of the products of bacterial action are not harmful. It is even possible that bacteria may in some ways be of service to the organism, as in attacking and breaking down indigestible substances, and producing from them materials which can be utilized by the body. In putrefaction, however, many harmful substances are produced, as above stated, and are responsible for the well-known ill effects of constipation. The material retained in the intestine putrefies, the products are absorbed into the blood, and give rise to headaches and various other symptoms causing discomfort.

Putrefaction is greatly favored by an alkaline reaction. Also most of the harmful substances resulting from putrefaction come from the proteins. Limiting the amount of protein in the diet and thoroughly chewing the food so that it may be digested quickly will greatly reduce putrefaction in the intestine.

Feces.-In every mixed meal there is some material which is indigestible and will not be attacked by the digestive enzymes. Connective tissuc, cellulose, etc., are examples of such substances. This material remains in the intestine and forms a part of the solid excreta, or feces. In addition, the feces contain enormous numbers of dead bacteria. Between one half and one fourth of the solid matter of the feces consists of dead bacteria. The feces also contain the waste material of the cells of the intestinal mucosa, and the residues of the digestive secretions. The total amount of solids excreted in the feces per day 
averages 15-25 grams. This amount may vary much with the nature of the food. The feces are colored chiefly by derivatives of the bile pigments, but these substances themselves do not occur here ordinarily. If the flow of bile into the intestine is inhibited, the feces are gray, due to the presence of fat and the absence of pigments. Many drugs color the feces black, green, yellow, etc. 


\section{CHAPTER X}

\section{ABSORPTION}

General.-In the preceding chapters we have followed the disintegration of the various foodstuffs in the different regions of the alimentary canal. By the combined or successive action of the digestive enzymes and other agents the widely varying carbohydrates, fats and proteins of the food are broken down into their simple building stones, monosaccharides, fatty acids and glycerine, and amino acids. Thus from the complex foods a mixture of simple substances is obtained. This mixture may vary from time to time in the relative amounts of the different building stones it contains, but the kinds of material it contains are fairly uniform. In a strict sense the digestion products are not yet in the body however. The alimentary canal is nothing more nor less than a tube or corridor which passes through the body. Through this corridor a great variety of materials is made to pass. These materials are torn to pieces, and from the resulting simpler substances the ever ready cells of the walls of the alimentary canal select and take up certain substances. This process is called absorption. As a matter of fact it has been much debated whether absorption is merely a physical diffusion of material through the walls of the alimentary tract, or whether it involves the activities of the cells making up the intestinal walls. Undoubtedly both of these factors come into play.

Practically no absorption takes place in the mouth, and but little in the stomach. There is some evidence that salts, monosaccharides and certain other substances may be absorbed to some extent from the stomach if they are present in high concentration, but this is of little importance normally.

The largest part of the absorption takes place in the small intestine. The surface area of the intestine is greatly increased 
by finger-like projections, called villi, which extend into the cavity of the intestine. The villi are supplied with blood vessels,-arteries, capillaries and veins, and each contains also a lacteal, or lymph vessel. The walls of the villi are very thin, so that only a thin membrane separates the food in the intestinal contents from the capillaries and lacteals. The cells of this membrane are alive, and whereas simple processes of diffusion probably account for a portion of the absorption of food, the living cells of the intestinal wall undoubtedly influence the process by taking up particular substances and passing them, or products made from them, on into the blood in the capillaries or into the lymph in the lacteals.

Absorption of Proteins.-Proteins are absorbed mainly in the form of amino acids. A portion of these are passed directly into the blood of the capillaries and thus enter the blood stream by which they are carried to the different tissues and cells. Possibly a portion of the amino acids are deaminized,- -that is, lose their amino groups in their passage through the intestinal wall. Little is known of the further fate of the residue left after splitting off the amino group. Perhaps it is burned by the cells of the body as fuel, or used to build up new substances in the cells. Amino acids have been obtained from the blood or shown to be there by various methods, so that there is no longer doubt that this is the form in which the proteins of the food reach the cells. This subject was long in dispute, for the amount of amino acids in the blood at any one time is so small that only recently has it been possible to prove that they are there at all.

Carbohydrate Absorption.-The monosaccharides glucose, levulose and galactose produced by the digestion of the carbohydrates are believed to pass into the blood of the capillaries and thence into the blood stream. Glucose is found regularly in the blood to the extent of $.08-.12 \%$. The liver takes up monosaccharides and builds them into glycogen, which serves as a reserve store of fuel. Glycogen is stored also in the muscles.

At times, if excessive amounts of maltose, lactose, or cane sugar are taken, these sugars may get into the blood. The mal- 
tose will be split and utilized at least in part, as the blood contains a maltase. Blood appears to contain little or no lactase or invertase, however, so that lactose or cane sugar which get into the blood are excreted into the duodenum, or in the urine.

Absorption of Fats.-Fats are absorbed mainly in the form of fatty acids and soaps. These are recombined with glycerine in the cells of the intestinal wall, and pass into the lacteals as neutral fat.

In the absorption of fats the bile plays an important role. If bile is excluded from the intestine, the absorption of fats is greatly diminished. Large amounts of fatty acids, soaps, and some fat appear in the feces, indicating that the fatty acids and soaps are absorbed very slightly in the absence of bile. Bile aids in the absorption process by its power of dissolving fatty acids and soaps. These soaps and fatty acids dissolved by the bile salts pass into the cells of the intestinal wall. Glycerine also is absorbed, and from this and the fatty acids, neutral fat is constructed. This passes for the most part into the lacteals, thence along the lymphatics and into the thoracic duct whence it is poured into the blood stream at the junction of the jugular and subclavian veins. The bile salts which have entered the blood with the fatty acids are picked out of the blood by the liver and returned to the bile.

During digestion, white corpuseles are known to gather in large numbers in the neighborhood of the intestine. Evidently they are concerned in some way in taking care of the absorbed foodstuff.s, but their rôle is as yet a matter of uncertainty.

Most of the absorption of digestive products takes place in the small intestine, so that little useful material is left by the time the digested food reaches the large intestine. In this region, however, there is a large absorption of water so that this valuable liquid is not wasted by excretion with the feces. 


\section{CHAPTER XI}

\section{URINE}

General.-The body is by no means a permanent structure if considered from the standpoint of the individual molecules of which it is composed. The tissues are constantly wearing out and being rebuilt in the process of wear and tear. In this process waste products are formed. For the maintenance of body temperature and the performance of mechanical work, substances are constantly being "burned" or oxidized in the body, both substances from the food, or from the organic reserves of the tissues themselves. Here also waste products are produced. The body must dispose of waste material. This is accomplished by way of the various excretions,--through skin, lungs and kidneys, and in the feces.

The skin excretes water and salts, and about a gram of nitrogen per day in various compounds. From the lungs much water and carbon dioxide are given off. The feces contain mainly undigested food residues, the dead bodies of intestinal bacteria and substances from the digestive secretions, but also some other materials which are excreted into the intestine. They contain 1-2 grams of nitrogen per day.

Most of the nitrogen excreted is given off in the urine however, and hence this excretion attracts especial interest; a study of the amounts and variations in the nitrogen elimination often will give valuable information as to what is going on in the body itself. This is not confined entirely to nitrogen compounds, for the urine contains various other substances of interest. Occasionally also, abnormal constituents appear, and by their presence or amount give information which is of great value to the physician. The study of the urine is thus of primary importance. 


\section{Physical Properties}

Volume.-The excretion of the various urine constituents varies considerably during the course of the 24 hour day. Except in the case of some pathological constituents, the mere presence of which is an indication of an abnormal condition, it is customary to make analysis of, and report the amounts of the substances found in the urine voided during a complete 24 hour period. Such a 24 hour specimen is most conveniently collected by discarding the first voiding in the morning, then collecting all urine voided during the day, and the first voiding of the following morning. The specimen should be preserved from spoiling by adding 5-10 c.c. of a $5 \%$ thymol solution in chloroform. More recently much work has been reported on the analysis of three-hour specimens.

The volume of such a sample varies through fairly wide limits. It is determined by the balance between the amount of water taken, and that excreted in other ways. Thus, loss by excessive sweating, by diarrhea, or vomiting, or in fever where evaporation from the skin is increased will cause a fall in the urine volume. On the other hand, drinking much water, or prevention of loss through the skin as on humid days when evaporation is low, will tend to increase the total volume of the urine. An average figure for adult men is 1,000-1,200 c.c. per day, in women somewhat less, but the volume from a perfectly healthy subject may be much lower (400-500 c.c. or less) or much higher (two to three liters or more). Children excrete less urine than adults, 600-700 c.c. being an average amount between the ages of 3 and 7 or 8 years. A new born infant excreted 17 c.c. the first day, and on the sixth day, 206 c.c. Vegetarians usually excrete a small volume.

In pathological conditions, such as fevers, or in kidney affections, the volumes may greatly diminish (oliguria), or no urine may be excreted at all. Such a condition is of course extreme. In the various forms of diabetes the volume may be very large; 
even 10 liters per day and greater volumes have been reported. Such a condition is known as polyuria.

Color, Transparency.-The color of normal urine ranges from a pale straw color to a dark brown. Ordinarily it is amber. The color is due to the presence of pigments,-urochrome and urobilin being of greatest importance. Uroerythrin may give normal urine or its sediments a reddish color. Dilute urines of large volume usually are pale in color, concentrated urines usually darker. The reaction of the specimen also affects the color, as acid urines are usually darker, alkaline urines lighter.

Various pathological colorings may occur,-thus blood pigment or its derivatives may cause a red or brown; bile pigments give the urine a dark brown, greenish, or greenish black color. In a condition known as alcaptonuria the urine turns dark on standing. It should be borne in mind that various drugs will color the urine, such as senna (yellow), tar preparations, salol, etc. (brown). Also madder, beets, or the analine dyes used in cheap candies may result in abnormal colorations.

The transparency of normal urine varies greatly. Fresh acid urine usually is clear or fairly so. If concentrated, it may be cloudy from a precipitate of urates or uric acid. This precipitate will dissolve on warming. On standing, a slight sediment usually settles out. This consists of various cells or cell debris, urates, uric acid and some other substances.

If the specimen is alkaline, it usually is cloudy from precipitated phosphates or carbonates of the alkaline earths. This cloudiness does not disappear on warming, but does on acidifying.

Pathological urine may be cloudy from mucin, epithelial cells, pus, blood, bacteria, etc.

To clear a cloudy specimen filtering often is sufficient. If the urine is alkaline, the addition of acetic acid may cause clearing.

Decolorizing may be brought about by adding charcoal, or permanganate or in other ways. The method chosen should not interfere with the analysis to be undertaken however. 
Albumin may be removed by the careful addition of acetic acid and boiling. Care should be taken (trial and error) to add just the right amount of acid, as excess or a deficient amount will result in incomplete precipitation of the protein.

Consistency, Odor, Taste.-The urine usually is thin and watery. It foams on shaking but the foam quickly disappears. If the urine is albuminous the foam may persist. If it contains much mucin or pus, the urine may be thickish in character.

The odor of fresh urine somewhat resembles that of a meat broth. It is quite characteristic. Little is known of the nature of the substances responsible for the odor. Recently a substance "urinod" has been reported. When urine is allowed to stand without a preservative, it quickly acquires a sharp ammoniacal odor. Decomposition has taken place. With a little practice, the analyst is able to detect when a urine has "spoiled," and become unsuitable for analysis. Albumin or pus urine, especially if old, often has a putrid odor. Ingested drugs or foods may cause an unusual odor,- - thus asparagus, or onions give the urine a disagreeable odor. After taking menthol, an odor of peppermint is observed.

The taste of normal urine usually is somewhat salty due to $\mathrm{NaCl}$. Diabetic urine may taste sweet, from the sugar present.

Specific Gravity.-Total Solids.-The specifie gravity of normal urine obviously depends upon the relation between the amount of total solids, and the volume of the urine. An average specimen will have a specific gravity of from 1.017-1.020, but it may fall to 1.010 or lower if the volume is large, or rise to 1.030 or higher if the volume is low or the total solids high. In new born infants the specific gravity is low, about 1.005-1.007.

In pathological conditions the volume and specific gravity may not vary inversely. Thus in diabetes mellitus a large volume is accompanied by a fairly high specific gravity, on account of the sugar present. In cases of albuminuria the volume may be low and the specific gravity low also.

Specific gravity usually is estimated by means of an areometer or urinometer. (See laboratory directions for urine.) 
The total solids excreted in the course of a day will vary considerably. Sixty grams is an average figure. The amount depends largely upon the amounts of sodium chloride and protein eaten, for $\mathrm{NaCl}$ and urea, the principal end product of protein metabolism in the body, make up the largest part of the total solids. (For the estimation of total solids see the laboratory directions.)

Optical Activity, Reducing Power, Fermentation, Etc.Normal urine is slightly levorotatory, since it contains minute traces of protein and of conjugated glucuronates, both of which rotate strongly to the left. It contains also minute traces of glucose, which is dextrorotatory, but the amount is too small to counteract the levorotatory substances.

Pathological urine may show strong rotation. If dextrose is present the rotation will be to the right. If protein or levulose are present in quantity, the rotation will be to the left, as is also the case if the urine contains large amounts of conjugated glucuronates, as it does after taking camphor, chloral hydrate, menthol and various other drugs.

Normal urine has a slight reducing power, due to its content of traces of sugar, of conjugated glucuronates, uric acid and other substances. The reduction is not sufficient to interfere in the ordinary Fehling test when small amounts of urine are used however, so that this test, as performed with normal urine is negative. In pathological urine, particularly carbohydrate urine, the reduction may be extensive, and is made use of to detect sugar.

On standing, urine may ferment in a variety of ways as the result of the activities of microorganisms. Ammoniacal fermentation is the most common. Micrococcus ureæ and B. ureæ decompose the urea, forming ammonia. The urine becomes alkaline, its color changes, and phosphates, etc., are precipitated. Dilute urine usually ferments quicker than a concentrated specimen. A strong acid reaction retards the process. Other types of fermentation also occur.

Pathological urines usually ferment quicker than normal 
specimens, as they furnish a good medium for the development of microorganisms. Occasionally, fermentation occurs in the bladder before the urine is voided. Various gases may be produced, the condition being known as pneumaturia.

If injected into the blood stream, urine has a toxic effect. This is due in part perhaps to certain alkaloidal substances present in small amounts, but also to disturbances in osmotic equilibrium.

Spectroscopic examination of the urine is sometimes valuable in the detection of blood or bile pigments.

Reaction.-The urine of a normal healthy individual may vary considerably in chemical reaction, - thus it may be acid, neutral or alkaline. The reaction depends primarily on the nature of the diet. On a meat (protein) diet, the urine is acid, on a vegetable (non-protein) diet the urine may be alkaline. Thus the urine of carnivora is acid, that of herbivora alkaline. If either class of animal is forced to eat the other class of material, the reaction of the urine changes accordingly. In starvation, the urine is acid, since an animal thus becomes carnivorous, living upon its own tissues. At the beginning of gastric digestion, the urine usually is alkaline, due to the abstraction of available $\mathrm{H}$-ions from the blood to form the gastric $\mathrm{HCl}$. Profuse sweating also may lower the acidity, since acid is carried out through the skin.

The effect of variations in diet upon the reaction of the urine is due to the products formed in the breaking down of food constituents. Proteins contain sulphur and phosphorus, which are transformed into sulphuric and phosphoric acids, and excreted largely in the form of acid salts and probably to a slight extent as the free acids. These two factors are mainly responsible for the acid reaction of urine, although there also are small amounts of various organic acids. The organic acids in vegetable products, on the other hand, are oxidized to $\mathrm{CO}_{2}$ in the body, a portion of which is excreted in the urine as carbonate. The hydrolysis of these salts is responsible for an alka- 
line reaction of urine. Ingesting organic acids such as citric, malic, etc., which are burned to carbonates, actually increases the alkalinity of the urine, thus bearing out the above conclusions.

As might be expected, the total titratable acidity of the urine varies greatly. An average 24 hour specimen on a mixed diet will require $150-400$ e.c. $\mathrm{N} / 10$ alkali to neutralize it, using phenolphthalein as indicator. These figures may be exceeded in either direction.

There appear to be no consistent and characteristic variations in acidity in disease.

The hydrogen-ion concentration of urine may be determined by the gas chain method, or by the use of a series of indicators such as that worked out by Henderson. The average value is $\mathrm{N} \times 10^{-6}$, but it ranges between $\mathrm{N} \times 10^{-5,1}$ and $\mathrm{N} \times 10^{-7.4}$.

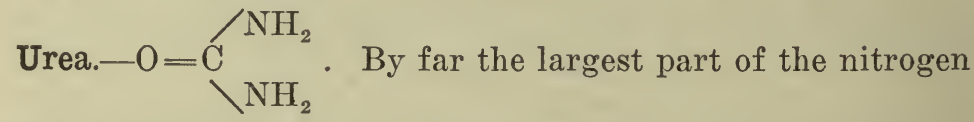

excreted by the body is in the form of urea. Other nitrogenous substances are uric acid, ammonia, creatinine and creatine, hippuric acid, allantoin, amino acids and a variety of other substances. Of the total nitrogen contained in all these substances, urea contains on an average $85-90 \%$. On a high protein diet this percentage may increase, and on a low protein diet it may fall even as low as $60 \%$. Also the total amount of urea varies with the amount of protein in the food. A variation of from 8 or 10 grams to 30 grams per day will cover most eases.

Urea is a colorless, odorless compound which crystallizes in long needles. It is almost tasteless, but produces a sensation of coolness when placed on the tongue. It is readily soluble in water and alcohol but insoluble in ether. With nitric or oxalic acid, it forms urea nitrate or oxalate respectively, one molecule of nitric uniting with one of urea, one of oxalic with two of urea. These salts crystallize easily and are useful in the isolation and purification of urea.

On heating dry urea, it decomposes, forming biuret, the sub- 
stanee for which the biuret test was named, cyanuric acid and other substances.
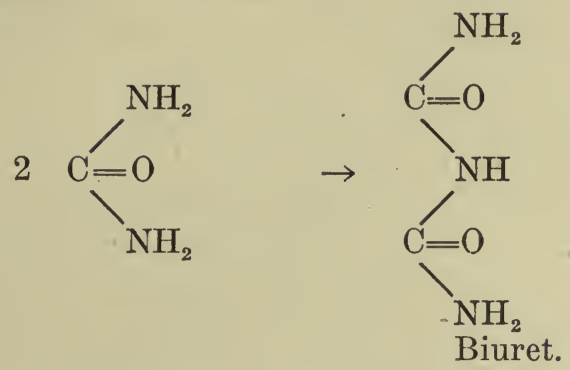

Urea is decomposed by nitrous acid, and by hypobromite; the nitrogen is liberated as the gas, and may be measured. Various methods for the quantitative estimation of urea depend upon these reactions. If heated to $153^{\circ} \mathrm{C}$. urea gives up its nitrogen as ammonia. This method also has been used in some of the most successful quantitative urea methods. An enzyme urease which is found in the soy bean, and elsewhere, has the property of decomposing urea, but no other nitrogenous constituent of the urine. The urea is converted into ammonium earbonate from which the ammonia easily may be liberated. This is the basis for the method of urea determination in general use at present.

The original source of urinary urea is the proteins of food and tissues. Proteins are made up of amino acids. In digestion they are split up into these compounds. Where and how is the amino acid nitrogen converted into urea? This is a question which has taken much labor to solve. A portion of the amino acids is deaminized by bacteria in the intestine, a portion possibly in their passage through the cells of the intestinal wall. The amino group is converted into ammonia which circulates in the blood as ammonium carbonate. It has been shown that an excised liver if perfused with blood containing ammonium carbonate will convert this substance into urea, but most of the amino acids pass into the blood after absorption. Amino acids undoubtedly are deaminized in the liver itself, and also in various other tissues, so that the supply of ammonium carbonate may come from very 
generally distributed regions. An attempt has been made to demonstrate that the manufacture of urea occurs only in the liver, but evidence does not bear out this assumption. Probably urea formation is a function of all cells of the body, and in 1922 Folin has published the conclusion that the liver has no specialized function in connection with deamination and urea formation. The liver cannot be removed from the body of a mammal without causing the death of the animal in a few hours. The liver may be shunted out of the circulation, however, by an operative procedure known as Eck's fistula. In an Eck's fistula, the portal vein is joined to the inferior vena eava. The portal vein beyond the fistula is then ligated. The blood from the intestine now no longer passes to the liver, but into the vena cava and thence to the heart. The liver still receives some blood by way of the hepatic artery but most of the blood does not pass through the liver. Under these circumstances, the amount of urea decreases greatly, with a corresponding increase in ammonia. It also has been observed that in various acute diseases of the liver or in injury to the liver cells after poisoning with eertain substances, a fall in urinary urea results.

A small proportion of the urinary urea may arise from the direct hydrolysis of one of the amino acid constituents of the proteins, viz. arginine. Inspection of the formula for this compound will demonstrate the ease of this process from a chemical standpoint. There probably also are various other minor sources of urea.

Urea has a distinct physiologic action, acting as a diuretic. Increase of urea in the blood increases the flow of urine. This is in harmony with the fact that on high protein diet, the volume of the urine also is high.

Uric Acid and Other Purine Derivatives.-Uric acid occurs in the urine in amounts ranging from about 0.3 to 1.2 grams per day. It makes up from 5-10\% of the total nitrogen of the urine. Notwithstanding its small amount, uric acid is of great interest, and long has attracted widespread attention because it occurs as a urinary sediment. On account of its insoluble character, it is 
deposited in the joints in gout and arthritis, and shows other interesting variations in disease and on varying diets.

In birds and reptiles uric acid is the chief nitrogenous excretion. The urine of these animals is semisolid, since it contains large amounts of uric acid crystals.

Uric acid is 2, 6, 8 trioxypurine and has the following formula:

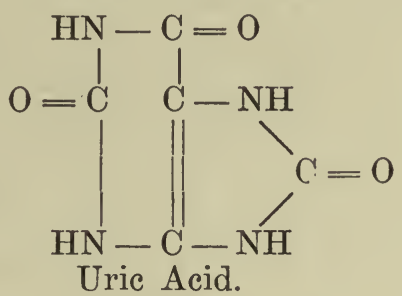

It is extremely insoluble and frequently precipitates from the urine as a crystalline sediment which usually is highly colored by other substances. The crystal form varies, but is frequently whetstone or gunboat shape. It dissolves fairly readily in alkalies, in boiling glycerol, and in concentrated sulphuric acid on slight warming without decomposition. It forms salts, those of sodium and ammonium often being present in urine sediment. Uric acid is easily decomposed in alkaline solution, and yields various products such as urea, dialuric acid, etc. It reduces Fehling's solution, and is readily oxidized by alkaline permanganate and other oxidizing agents. Alloxan, allantoin, oxalic and carbonic acids are among the oxidation products of uric acid.

If a crystal of uric acid is moistened with concentrated $\mathrm{HNO}_{3}$ and evaporated to dryness on the water-bath a reddish spot remains, which becomes a deeper red on the addition of ammonia. This is known as the murexide test. Other purines (xanthine and guanine) give this test, but may be distinguished from uric acid by the fact that the red color persists on warming, whereas that from uric acid disappears on warming. The red substance from uric acid is ammonium purpurate.

The source of the uric acid was long a matter of uncertainty. We now know, however, that it is derived from the purine por- 
tion of nucleic acid. Thus the nucleoproteins are the ultimate source. This fact increases interest in uric acid, since evidently it is a product of the breaking down or catabolism of nuclear material. The purine bases, adenine and guanine, are constituents of nucleic acid. In the body these compounds are oxidized to hypoxanthine and xanthine, and these latter substances to uric acid. These purines may come from the breaking down of nuclear material in the food, or in the tissues themselves. This fact may be demonstrated, for if glandular material such as sweet breads (pancreas) is fed, there is an increase in the uric acid of the urine. Since this uric acid has an origin outside the tissues, and comes from material which probably is at no time a constituent of the cells, it is called exogenous uric acid, i. e., coming from without. It is interesting that all of the ingested purines are not recovered in the urine as uric acid. Evidently a portion either is not absorbed, or is destroyed in the body. If an animal is kept upon a purine free diet, uric acid does not disappear from the urine; from 0.3-0.5 grams a day still is excreted. This must come from the nuclear material of the tissues themselves, and hence is called endogenous uric acid, i.e., coming from within. There is a possibility that at least a portion of this uric acid comes from the nuclear material of dead bacteria in the intestine, but it is generally considered to arise mainly in the catabolism of the tissue nucleins.

The transformation of the purines, adenine and guanine, into uric acid involves several steps. Quite recently this process has been carefully studied, and enzymes have been found in various tissues which possess the property of carrying out all the intermediate stages of the process.

In most mammals an enzyme uricase occurs which has the power of destroying uric acid,--allantoin being one of the chief products. This enzyme has not been found in man, however, and it is probable that man has lost the power of destroying uric acid. To be sure, if uric acid is given to a human being by mouth, only a small portion of it appears in the urine. This fact is still unexplained. Perhaps intestinal bacteria are responsible for its destruction. 
The question has been raised, ean the body build up its own purines (and from these its nucleoproteins) from non-purine substances? This question is as yet unanswered. In birds and reptiles the synthesis of uric acid is a well known fact. In invertebrates also this synthesis takes place. It would be curious if only mammals lacked this power. We know in fact that in very young mammals living on milk, which contains only traces of purine, large amounts of nuclear material are built up, apparently from non-purine sources. But the question is still unsettled, and awaits further evidence.

The variations in the amount of uric acid in disease are influenced by the amount of destruction of nuclear material in the body. During recovery from pneumonia, when the transudates containing large numbers of leucocytes are being reabsorbed, in leucemia where the number of leucocytes in the blood is greatly increased, after severe burns which have caused the disintegration of much tissue and in pregnancy when there is increased nuclear metabolism, an increase in uric acid is observed. In gout and arthritis uric acid is deposited in the joints causing much pain and inconvenience. It is a debated question, however, as to whether this is the cause or the effect of the disease.

Other purines occur in the urine in small quantities. Also caffein, theobromine and other members of the group which occur in coffee, cocoa, etc., may be present. The quantitative estimation of uric acid is described in the laboratory directions.

\section{Hippuric Acid.-}

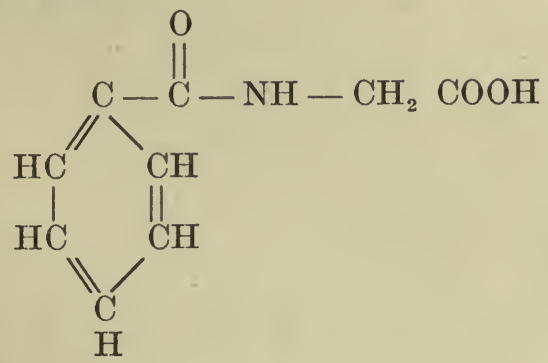


Hippuric acid is found in large amounts in the urine of herbivora. Less than a gram a day usually is present in the urine of man. It is interesting, for it is a substance formed by the union of benzoic acid and glycocoll. The former may be formed in the body, or may be ingested with the food, as it occurs in various fruits and vegetables, particularly in cranberries. The formation of hippuric acid apparently is a protective measure, as benzoic acid is considerably more toxic than hippuric. The benzoic is conjugated with glycocoll, which comes in part from the destruction of protein tissue or food, but also may be synthesized by the body. The hippuric acid is then excreted, and the more dangerous benzoic acid thus removed.

Ammonia.-The urine always contains small amounts of ammonia, usually less than a gram a day. Ammonia is used by the body to neutralize acids which are not oxidized and destroyed by the body. The taking of a mineral acid thus will cause an increase in urinary ammonia. This occurs also in diabetes, where aceto acetic acid and $\beta$-oxybutyric acid are produced and not further oxidized in the organism. They are excreted in the form chiefly of their ammonium salts. The source of the ammonia is largely the split-off amino groups liberated in the destruction of amino acids when proteins are destroyed. Most of this ammonia is built into urea, but a portion is excreted as such. For the quantitative estimation of ammonia, see the laboratory directions.

\section{Creatinine and Creatine.-}<smiles>CCN1CC(=O)NC1=O</smiles><smiles>CCN(CC(=O)O)C(=N)N</smiles>

Creatinine and creatine are two closely related compounds 
occurring in the urine. Creatinine is the anhydride of creatine. It occurs in the urine of adults in amounts ranging from 1-2 grams a day. On a diet which contains no creatinine, the amount is quite independent of the amount of protein in the food. It is thus evident that it is not an end product of the metabolism of food proteins. If creatinine is present in the food, however, (it is found in meats, meat extracts, etc.), almost all of the amount ingested reappears in the urine, and thus evidently is not destroyed in the body. On a creatinine free diet, the amount of creatinine in the urine is remarkably constant for each individual,--about 7-11 mg. of creatinine nitrogen per kilo body weight per day being excreted. This value is called the creatinine coefficient.

Creatine is found in the urine of children, of women during pregnancy, menstruation and after childbirth, and in the urine of adult men and women during fasting. It also occurs in the urine during carbohydrate starvation, and in diabetes. At such times the amount of creatinine decreases, the total of creatine + creatinine remaining about constant.

If creatine is taken by mouth, little or no increase in urinary creatinine or creatine occurs, a very singular fact. Creatine occurs as a constituent of most organs, and of voluntary muscle. There are about 120 grams of creatine in the body of an average adult. Creatinine also may be obtained from muscles.

The fact that the creatinine excretion is independent of the protein and creatine intake, that it varies with age and sex, and shows a fairly constant value in each individual has led to the conclusion of Folin that creatinine is a product and index of the endogenous metabolism of the tissues. The source of the urinary creatinine is probably the creatine of the tissues. The formation of creatinine appears to be independent of muscular work, as no increase is observed after exercise. Just what governs the formation of creatinine is still uncertain. The excretion of creatinine is greater during the day than at night, so that it has been suggested its formation may depend in some way on the maintenance of muscle tone, but this point is as yet undecided. 
The whole subject of the rôle and formation of the creatine of the tissues and the formation of creatinine is still in a very unsatisfactory state, although it appears evident that these substances are intimately connected with endogenous tissue metabolism itself,-with the actual wear and tear of the tissue substance.

Creatinine occurs in the blood, and increases in amount in nephritis. A value of $5 \mathrm{mg}$. per 100 c.c. blood is called the fatal ratio, since, if it reaches this level the patient rarely recovers.

\section{Inorganic Constituents}

The urine contains a variety of inorganic constituents such as chlorides, sulphates, phosphates, carbonates, etc., of sodium, potassium, calcium and magnesium. In addition, various others occur. There are traces of nitrates, believed to come mainly from nitrates in the drinking water. Iron also is found in traces, about $8-10 \mathrm{mg}$. per day. It probably is both in inorganic and organic compounds. Fluorides, silicic acid and other substances also occur, as well as accidental constituents taken with the food.

Chlorides.-Of the inorganic constituents, chlorides make up the largest part. Sodium chloride is present in greatest amount, and the total chlorides of the urine usually are reported as "sodium chloride." The total amount averages 10-15 grams of sodium chloride a day, but it may vary greatly, the variation depending chiefly on the amount of "salt" in the diet. Drinking much water will increase the chloride output. The excretion is greater during activity than at night. The amount decreases in some diseases, as during the formation of exudates in pneumonia. When reabsorption takes place after the crisis, the retained chloride reappears in the urine. It is interesting that sodium chloride is the only salt present in an ordinary mixed diet in amounts insufficient for the body's needs. It must be added to the food. The well known salt craving of herbivorous animals 
is an example of this. Carnivorous animals obtain enough salt from the bodies of the animals they devour.

Some chlorine may be present in the urine in organic combination, but if so, only in traces. No chlorine-containing organic constituent of the body is known. The quantitative estimation of chlorides is given in the laboratory directions.

Phosphates.-The urine contains about 0.5-1.2 grams phosphorus per day, but none of this is in the form of free phosphorus. It all is in oxidized form as phosphates, a portion of which are present as mono- or di-sodium, potassium, calcium or magnesium phosphates, some probably as free phosphoric acid, and some in ester-like compounds such as glycero-phosphoric acid, etc. The amount excreted is increased by a protein diet, since the phosphates of the nucleoproteins, lecithin, the phosphoproteins, etc., are the main source of urinary phosphates. Some inorganic phosphates also occur in the food as such. The amount in the urine also is influenced by the fact that phosphates are excreted in the feces. This is due partly to failure to absorb them, but also to the fact that phosphates are excreted into the intestine and eliminated in the feces chiefly as calcium phosphate. On an average, about $50-65 \%$ of the total phosphorus is excreted in the urine, the remainder in the feces. Constipation causes increase in urine phosphorus, and decrease in that in the feces. Diarrhea has the opposite effect. The phosphates of the urine are sometimes differentiated into alkali phosphates, alkaline earth phosphates, and organically combined phosphates.

In pathological conditions the phosphorus excretion is increased when there is increased destruction of nuclear material as in leucemia. Also in starvation the bone tissue is gradually drawn upon for fuel, and increased phosphate elimination results. The parathyroid glands also appear to be connected in some way, as yet not understood, with phosphate excretion.

Sulphates.-Sulphur occurs in the urine in various forms. Three classes usually are recognized, inorganic sulphates, ethereal sulphates and unoxidized or "neutral" sulphur. The 
total amount of sulphur excreted per day, is variable, but usually is somewhat less than a gram. The chief source of urinary sulphate is the sulphur of the protein molecule. Most of this is oxidized in the body, so that $75-80 \%$ of the total sulphur is present as inorganic sulphate. The remainder is made up of organically bound sulphate, and of a mixture of traces of several compounds containing sulphur in unoxidized form, such as sulphocyanate (which we already have met as a constituent of saliva), cystin, sulphides, etc.

Since the proteins are the chief source of urine sulphur, there is a general parallelism between the nitrogen and sulphur excretion, which often runs about as follows: $\mathrm{N}: \mathrm{H}_{2} \mathrm{SO}_{4}=5: 1$. But this is by no means a constant ratio.

The ethereal sulphates are compounds in which sulphuric acid is conjugated with various phenols produced from the proteins by putrefaction in the intestine. From tryptophane, indol and skatol are produced and oxidized in the body to indoxyl and skatoxyl which are then combined with sulphuric acid to make ethereal sulphates. The synthesis of these compounds, as in the case of the conjugated glucuromates, is a protective measure, the toxic phenols being converted into relatively non-toxic ethereal sulphates.

Variations in the amount of protein in the diet cause relative as well as absolute variations in the different sulphur constituents. If the amount of protein in the food is decreased, the inorganic sulphates fall both in amount and in per cent of the total sulphur which they represent. The amount of neutral sulphur remains fairly constant, so that the per cent rises. Thus neutral sulphur, like creatinine, probably is largely a product of endogenous tissue metabolism, since it is independent of the protein intake in the food. Neutral sulphur is increased in eystinuria, by chloroform and after taking cyanides, which are converted into sulphocyanate. Putrefaction in the intestine decreases in amount on a low protein diet, so that ethereal sulphates decrease, but usually not so rapidly as the total sulphur, so the percentage increases somewhat. 
Carbonates are found often to considerable extent in the urine. An alkaline reaction of the urine may be due largely to carbonates. They often precipitate in alkaline urine and are thus found in the sediment.

Sodium, potassium, calcium and magnesium are present chiefly as chlorides, sulphates, phosphates and carbonates. The amounts depend much on the quantity of each present in the food. In the case of calcium and magnesium much may be lost in the feces. About 1 gram a day of the phosphates of these two metals is found in the urine. Two to 4 or 5 grams each of sodium and potassium may be excreted daily.

\section{Pathological Constituents of the Urine}

The most frequent pathological constituents of the urine are proteins, carbohydrates, acetone bodies, casts, bacteria, etc. The study of pathological urine lies properly in the field of pathology. A short review of the kinds of the above substances occurring most frequently in pathological urine has been included in the laboratory chapter on the urine, together with the most important or convenient methods for detecting and estimating them. Certain anomalies of metabolism leading to the appearance of these substances in urine will be discussed in the chapter on metabolism. 


\section{CHAPTER XII}

\section{METABOLISM}

General.-The body in some respects resembles an engine,it requires fuel to carry out its various activities. This fuel is "burned" or oxidized, and heat or mechanical work results. In the process, waste products are formed. In addition, the individual parts of the structure are continually breaking down and being repaired. All this we know from a comparison of the "fuel" or food substances ingested, and the waste products eliminated. All evidence points to the fact that extensive chemical activity is going on in the tissues. Materials are torn to pieces, chemically speaking, other substances are built up. The field covering the processes going on in the tissues is known as metabolism. This does not include digestion, for strictly speaking, the contents of the alimentary tract are not in the body, at least not in the tissues, but only in a passageway or tube running through the body. The study of metabolism covers the history of the foodstuffs from the time of their absorption to the point where they, or the products formed from them are excreted from the body. We may differentiate various fields of metabolism, such as that of proteins, carbohydrates, fats or inorganic materials, and also some other fields, such as energy exchange. In addition, we may study the general metabolism of a localized area, such as metabolism of the muscles, liver, etc.

It is obviously impossible to enter the tissues and observe what is going on in them. Our observations must be made in indirect ways, and conclusions drawn as well as the case permits. Various methods for the study of metabolism are employed. For example, from variations in the constituents of the urine and the amounts of the different substances present, from observations made on excised surviving organs through which blood or some 
similar fluid is kept circulating, from a study of the relationship between oxygen consumed and carbon dioxide eliminated (respiratory quotient), and from measurements of the heat produced by the body much valuable information has been obtained about the processes going on in the tissues.

Protein Metabolism.-The metabolism of proteins, and problems connected with this field make up one of the most important phases of metabolism. The reason for this is not difficult to divine. Proteins make up the bulk of the solid material of living tissue. Without an adequate supply of them in the diet, an animal will die, even though it be given all it will eat of the other food substances such at fats, carbohydrates, salts, etc. Evidently proteins have some rôle to play which cannot be filled by the other classes of foods. Since the other foods are good fuels, and are easily burned in the body, the importance of the proteins seems to be connected with furnishing building or repair material to the tissues, or supplying certain chemical groupings required for the manufacture of some vitally important products manufactured in the body itself. It will be of interest to follow the proteins and the products formed from them in their passage through the organism.

In the digestive tract proteins are broken down by enzymes into amino acids and in this form they are absorbed and pass into the capillaries of the villi, and thence into the general circulation by way of the portal vein. Until a few years ago it was a matter of lively dispute whether the digestive products of the proteins entered the blood in the form of amino acids, of proteoses and peptones, or were rebuilt in the intestinal wall to form proteins. It had never been possible to find amino acids or proteoses and peptones in the blood, so that many investigators believed these products were rebuilt into protein before passing into the blood. One by one, pieces of evidence have accumulated to settle this question, and we now know that normally the greater part at least of the amino acids produced in digestion gets into the blood as such, and is transported to the tissues. It has been shown that if proteoses and peptones are injected into 
the blood, enzymes appear in the blood stream eapable of breaking them down. No such enzymes occur in the blood normally. Obviously then, proteoses and peptones normally are not present in the blood. The chief difficulty in showing that free amino acids are present in the blood lay in the complex character of that liquid, and its high content of protein. During the digestion of a protein meal the amount of amino acids absorbed at any one time would be very small. Also the flow of the blood is rapid, so that any increase in the total nitrogen of the blood would be slight and thus difficult to detect in the presence of so much other nitrogenous material. Folin and his co-workers showed an increase in the non-protein nitrogen of the blood after a protein meal. Abderhalden demonstrated that amino acids are present in the blood, by using very large volumes of blood. Van Slyke, by his now well known method for determining small amounts of $\propto$ amino groups, showed a marked increase after a protein meal, and Abel and his co-workers by passing the circulating blood of a living animal through a diffusion device succeeded in isolating considerable quantities of amino acids from the blood. The problem was thus solved. The proteins of the food are reduced to amino acids in the digestive tract, are absorbed as such, and transported to the tissues. It has been reported that a portion of the amino acids lose their amino groups in the intestinal wall. If this is the case, the amount so destroyed is extremely small.

The further fate of the amino acids still is very obscure. They are taken up by the tissues from the blood. Van Slyke has shown that the amino acid content of the tissues is greatly increased after a protein meal. Probably certain of the amino acids are used for the repair of cell proteins, or in the manufacture of cell products. There appears to be little or no storage of protein material, however, for the nitrogen from ingested protein reappears in the urine within twenty-four hours or so after taking. The protein structures of the body appear to increase only in growth, in recovery after a wasting disease, or 
in connection with increased activity, and not as the result of taking an excessive supply of this material.

Various modes of decomposition are possible in the destruction of amino acids. The amino group might be removed, leaving a fatty acid, or oxidation might result in the formation of an oxyacid or a keto acid; thus from alanine, propionic acid, lactic acid, or pyruvic acid would result.
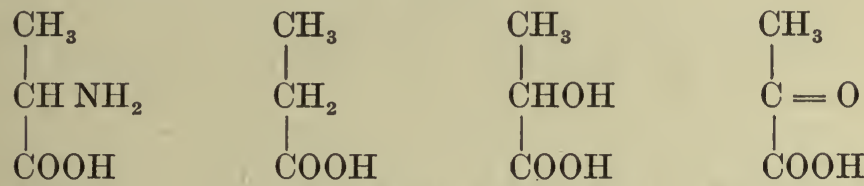

Alanine

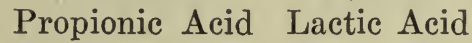

Pyruvic Acid

Enzymes are known which can produce any one of these types of reaction. These products might then be further oxidized to $\mathrm{CO}_{2}$ and $\mathrm{H}_{2} \mathrm{O}$, or they or their derivatives could be utilized in the construction of other substances needed by the tissues. Pyruvic acid and the corresponding aldehyde undoubtedly play an important part in the destruction of more than one amino acid, and the resynthesis of other substances. The property of deaminizing amino acids undoubtedly is possessed by all cells.

Amount of Protein Required.-Nitrogen Balance.-Since protein is indispensable, it is of interest to know how much protein is needed by the body. Protein is an expensive article of diet. It would be of economic advantage to eat no more protein than necessary for the maximum efficiency of the body. Excess protein is not stored for future use, but is broken down, and its nitrogen excreted mainly as urea within a comparatively short period. Ordinarily an adult is in a condition known as nitrogen equilibrium. That is, he excretes just the amount of nitrogen that he takes in his food. His body is neither storing up nor depleting its store of nitrogenous material or protein. Such a condition of exact balance does not always exist, however. In a growing child or in an adult during recovery from a wasting disease, or even during periods of "training" or unwonted exercise, the body may lay on protein tissue,-muscle for 
example. In this ease the individual will be exereting less nitrogen than he reeeives in his foods. He is said to be in positive nitrogen balance since he is laying up nitrogen compounds. In wasting disease, in starvation, or on insufficient protein diet the loss of nitrogen may be greater than the intake in the food. This condition is known as negative nitrogen balanee.

It is a curious fact that nitrogen balance may be maintained on widely varying levels without any apparent inconvenience. The problem of the necessary amount of protein in the diet thus resolves itself into the problem,-on what amount of protein ean an individual be kept in nitrogen equilibrium; a further phase of the question will be to determine whether minimum protein is desirable from a physiologic and general point of view.

If a man is consuming a diet containing 10 grams of protein nitrogen, and is on nitrogen equilibrium at that level, suppose his protein ration to be doubled, so that he will be taking 20 grams of protein nitrogen, and that he continue on this diet. The first day he will exerete considerably more than 10 grams of nitrogen, but not the entire 20 grams. The second day he will excrete still more, and on the third or fourth day he again will be in nitrogen equilibrium, but now on 20 grams nitrogen. If this is now eut to 10 grams, the process is reversed, and in three or four days he will be in equilibrium again at the lower level.

An ardent advocate of a low protein diet was Horace Fletcher, an American who long suffered from ill health. $\mathrm{He}$ greatly reduced both the total quantity of food taken, and the amount of protein which it contained. The results were so satisfactory that a study of the problem was undertaken by various investigators. Chittenden earried out a long series of metabolism experiments on several men. Folin's report of the case of Dr. van Sommeren indicated the effects on the quantitative composition of the urine of a low protein diet. (Folin's "30 Normal Urines," an analysis of 30 24-hour specimens was the first complete study of the 24-hour output of normal urine eonstituents. This furnishes a standard for comparison.) These results already have been referred to. Folin himself lived on a starch 
and cream diet containing only about 6 grams of nitrogen a day for several days. Chittenden drew the conclusion that the most desirable amount of protein for an average sized adult is 40 grams per day or somewhat over 6 grams of nitrogen. This is much less than the standards previously recommended. Voit, for example, basing his conclusions on the amount of protein consumed per capita in several European cities, recommended 118 grams of protein (19 grams nitrogen) as the proper amount, and most other standards were of this general magnitude.

It has been shown that nitrogen equilibrium may be established on a level even lower than that of Chittenden. Thus Thomas reduced his nitrogen to 2.2 grams a day (about 15-20 grams protein). In fact on a low protein intake the protein evidently is used more economically by the body. But the problem remains,-is so low a level of protein intake desirable or safe? Some evidence has accumulated on this point. Certain tribes in India who live on low protein diet have been observed to be less efficient and to possess less endurance than neighboring tribes of meat eaters. Races living in cold climates usually live on a high protein diet, and such races display great endurance. The whole experience of the human race seems to have tended toward a fairly high protein diet. If low protein intake resulted in greater efficiency, it is only reasonable to suppose that races or nations living on low protein rations would have dominated over others. From the experiments of Chittenden and others it appears possible to maintain a group of individuals for several months on a low protcin diet, apparently with good results and increased efficiency and health. It might be suggested, however, that such favorable results may be due in part to the carefully regulated living, wisely directed exercise and wholesome food of the subjects, for it is an unfortunate but incontrovertable fact that many of us are sadly lax in the ordering of such important factors in our existence as exercise, proper food, and general healthful living. From experiments of but a few months' duration it is dangerous to draw conclusions of so im- 
portant and far-reaching a nature. It is possible that on minimum protein intake, reserve stores of important but seldom needed materials might be seriously depleted, thus reducing the body's power of resisting disease or meeting emergency demands. In this connection, the work of Haecker is most interesting. A herd of eattle was kept for about three years on low protein diet. The first two years all went well. In the third year the animals showed lessened resistance to the inroads of disease, and finally became so ill that the experiment was discontinued.

In summary, perhaps the older standards of about 120 grams protein are unnecessarily high. A compromise on perhaps 90 grams of protein for the average adult has been suggested as the most satisfactory solution of the problem.

Recently (1920) Sherman has reported 0.633-0.637 grams protein per day per kilo of body weight as adequate for maintenance in man.

The fact that nitrogen equilibrium can be maintained on so low a level as 2-3 grams $\mathrm{N}$ a day makes it seem possible that the breaking down of the protein tissues takes place to a much smaller extent than was supposed. Another and a more probable explanation of this fact is that much of the material produced in the destruction of protein tissues is used again in the body to rebuild the destroyed tissues.

The maximum amount of protein on which nitrogen balance may be maintained is of little practical interest. It will be limited by the ability of the organism to absorb protein products from the digestive tract. Excess protein is simply destroyed in the organism. In addition to the economic disadvantage of so expensive a diet, unnecessary strain is put on the excretory organs in disposing of the excess of urea and other end products formed. It is an interesting fact that increased muscular activity does not increase nitrogen excretion if the animal is well nourished. The animal burns carbohydrates and fats, and destroys no more protein.

Thus far we have spoken only of protein need. In so doing, we really are considering amino acid need, for the proteins are 
broken down to amino acids in digestion. Since proteins contain widely varying proportions of the different amino acids, and a given protein often lacks one or more of these compounds, it is not surprising that different proteins vary greatly in their usefulness and adequacy in the diet. The amount of protein required thus is dependent on the kind of protein. If the body requires a definite amount of a particular amino acid, that amino acid must be present in the food protein unless the body is capable of constructing it in its own workshop from other material such as ammonia and residues of other amino acids, of carbohydrates or other substances. Most interesting results have been obtained in this field.

Gelatine lacks tyrosine and tryptophane. Although gelatine is a protein, if an otherwise adequate diet is fed containing gelatine as the sole protein constituent, the animal will die as surely as if he were receiving no protein. Addition of these missing amino acids to the diet, either directly or by adding a protein which contains them will remedy the difficulty, and make the diet adequate. Osborne and Mendel, and McCollum have contributed greatly to our knowledge in this field. Zein, a protein from corn, contains no tryptophane or lysine. On an otherwise adequate diet containing zein as its sole protein, a young animal declines and dies. On adding tryptophane to such a diet, the animal no longer loses weight. It maintains about the same body weight. Apparently tryptophane is necessary for body maintenance. But on such a diet the animal does not grow. If lysine be added along with the tryptophane, the animal now grows almost at a normal rate. Lysine thus appears to be necessary for body growth. From the above discussion it is evident that the body cannot synthesize tyrosine, tryptophane, or lysine, at least in amounts sufficient for its needs and this is also true of cystine. Some of the amino acids apparently can be built up by the body. Thus after feeding benzoic acid, glycocoll will be excreted in hippuric acid in quantities too large to be accounted for by the available glycocoll in the organism. Probably some other amino acids, among them proline, also can be built up by the body. 
The fact that maintenance is possible on a zein-tryptophane protein ration (lacks lysine) but not growth, which would entail formation of new protein, may indicate that the breaking down of proteins in the tissues is not general, but for the purpose of supplying some amino acid required for the manufacture of a necessary substance. Otherwise, it would be difficult to understand why the body can build enough protein to repair that broken down, but no more for growth unless lysine also is fed.

The problem of protein requirement thus is resolved into a problem of amino acid requirement, limited on the one hand by the body's needs, and on the other hand by its ability to construct amino acids itself.

Since amino acids are the real requirement of the body, one would expect it to be possible to supply an animal's protein requirement solely by a mixture of amino acids. Such experiments have been attempted and their results point in general to this conclusion. It may be noted, however, that the difficulty of experiments of this sort is much increased by the unpalatable nature of such a mixture, so that positive results have not been always the rule. Dogs fed on such mixtures often refuse to eat, and if fed by a stomach tube, frequently vomit. In a German laboratory, an attempt by one of the assistants to live for a period upon a mixture of amino acids and the building stones of the fats and carbohydrates as well, was abandoned after the first attempted meal as a result of the unappetizing nature of the mixture. By injection of protein decomposition products into the blood, it has been possible to maintain an animal on nitrogen equilibrium.

The rôle played by the individual amino acids in the organism is still obscure. Probably certain of them are used as repair material for the tissue proteins. Others may be employed for the manufacture of important products of internal secretion. It is probable that the active substance of the internal secretion of the thyroid, thyroxin, is made from tryptophane, whereas adrenalin very probably is made from tyrosine. Such acids as are not required, undoubtedly are deaminized and the residues 
either used for constructing other compounds, or burned as fuel, as are the fats and carbohydrates.

The composition of the proteins of the tissues is remarkably constant, and quite independent of the nature of the food protein. A horse was bled to remove much blood protein. This the body was obliged to replace. The horse was fed at the time on gliadin, a grain protein containing a large percentage of glutamic acid. The blood proteins contain less than a quarter as much glutamic acid as does gliadin, but they were regenerated, and showed no variation from their normal composition.

The proteins of each individual tissue, and of each different animal undoubtedly are specific, that is those from different sources differ slightly in composition. It has been suggested that possibly they are the substances which transmit species characteristics, since they vary in different animals, whereas most of the other body compounds, such as salts, carbohydrates enzymes, nucleic acids, etc., are practically identical in different animals. This is in the realm of speculation, however.

Carbohydrate Metabolism.-A field of interest second only to that of protein metabolism is the metabolism of carbohydrates. Carbohydrates make up a large and important part of our food, and although they play a much less conspicuous rôle as constituents of body tissue, they are excellent fuels, and furnish the body with a considerable proportion of the material burned for the maintenance of body temperature and the performance of mechanical work.

The various carbohydrates of the food such as starches, dextrins, disaccharides, etc., are reduced to monosaccharides by the digestive enzymes, and as such are absorbed and pass into the blood stream. What is their further fate? The monosaccharides have been shown to be present in the blood stream as such, and not combined or united with any other substance, at least in more than the most unstable union. Dialysis of blood against dextrose solutions of various strengths has shown that the blood sugar evidently is free.

The blood from the intestine is gathered into the portal vein 
and passes to the liver and here begins the story of its utilization in the body. Claude Bernard, a French scientist, discovered in the liver a substance to which he gave the name glycogen. This substance is a polysaccharide, and on hydrolysis yields glucose. Glycogen occurs in places other than the liver, for example, the muscles also may contain it. It appears that glycogen is a reserve supply material which serves to store up sugar for the organism. In case of need, the glycogen is broken down, and furnishes the tissues with a supply of glucose for fuel. The amount of glycogen which the liver and muscles can store is limited, however. About 150 grams is the maximum amount which either of these tissues can lay up. Since glycogen is a reserve fuel for the body, it is called upon in case of need and conditions requiring the body to call on its reserves will cause a diminution in the glycogen. Liver glycogen appears to be particularly available for immediate use. Hard work, starvation, exposure to cold and various other conditions will greatly reduce the amount of glycogen in the liver, and also in the muscles. The sources from which glycogen may be built up will be discussed at a later point in this chapter.

The breaking down of liver glycogen has been shown to be influenced by a center in the medulla. Injury to this center, which may be brought about in rabbits by forcing a steel pencil into the brain in front of the occipital prominence in such a way that the floor of the fourth ventricle is pierced, causes sugar to appear in the urine. The percentage of sugar in the blood rises much above normal. If the animal is killed and the liver examined, it will be found to contain only a trace of glycogen. Evidently impulses from this region of the medulla cause the conversion of liver glycogen into glucose. Overstimulation of this center results in flooding the blood with sugar. The kidneys are so regulated that they keep a constant percentage of sugar in the blood. Any excess over this amount is excreted in the urine. It is evident that puncture of the medulla does not destroy the sugar center, but only irritates it, for the glycosuria is temporary, and passes off after a short time. The center is con- 
sidered to be a true reflex center, and may be stimulated by afferent impulses. Thus if the vagus is severed and the central end stimulated, sugar appears in the urine. If the splanchnics are cut, there is no glycosuria after puncture. Thus evidently, it is only liver glycogen which is affected by puncture, and the impulses are carried to the liver by the splanchnics. Glycosuria produced by "diabetic puncture" is known as "puncture diabetes."

A second factor which apparently is connected with the control of liver glycogen is the secretion of the suprarenals, adrenaline. If adrenaline is injected, an increase of blood sugar occurs, which has its source in liver glycogen, since no glycosuria results if the glycogen supply of the liver has been exhausted. It has been suggested that the action of the sugar center in the medulla is by way of the suprarenals. This problem is still in doubt; it is probable that the sugar center works by direct stimulation of the liver cells, but is aided and supplemented by the adrenaline secreted from the suprarenals.

Recently Langfelt has published interesting studies on liver diastase, the enzyme which brings about glycogenolysis (breaking down of glycogen). This enzyme, in the form of its chlorine derivative, acts best at a $\mathrm{pH}$ of 6.8 , and in the form of its phosphate derivative at a $\mathrm{pH}$ of 6.2 . In the presence of adrenaline the optimum $\mathrm{pH}$ was 7.73 . The $\mathrm{pH}$ of the blood is about 7.33. Although the $\mathrm{pH}$ of the tissues is not known, they are considered to be a little less alkaline than the blood. On this basis, the presence of adrenaline would affect the diastase in such a way that its optimum $\mathrm{pH}$ came nearer the $\mathrm{pH}$ of the tissues, its activity would be increased and glycogen would be broken down more rapidly into glucose. These findings agree well with the known fact that injection of adrenaline causes an increase of blood sugar.

The pancreas has been shown to play an important part in the utilization of sugar by the body. This is connected with the burning of the sugar as fuel and also with the storage of glycogen in the liver. If the pancreas of an animal is removed, 
sugar appears in the urine, and the amount of blood sugar rises much above the normal. Very little liver glyeogen is stored up. The explanation of these facts occupied a great many years, and many points are still obscure. It was found that if even a small portion of pancreas tissue is grafted under the skin and the blood vessels and nerves of the fragment left intact, extirpation of the remainder of the gland does not cause glycosuria. The action of the pancreas evidently is independent of the pancreatic juice secreted into the intestine. If this transplanted portion of the pancreas subsequently is removed, hyperglycemia (excess sugar in the blood) and glycosuria appear. Only within the last few years has fairly conclusive evidence been obtained that the pancreas produces a substance which is given off into the blood,-an "internal secretion," without which the tissues are unable to use glucose. If this substance is lacking, the amount of glucose in the blood increases, and the excess is excreted in the urine. It often has been affirmed that the "Islands of Langerhans," small groups of certain cells present in the pancreas, are responsible for the production of this important internal secretion. The evidence for this is not conclusive, however, and it is still uncertain where in the gland the substance is formed.

Still another factor is concerned in the control of glucose utilization in the body, and that is the influence of the kidneys. It has been stated that the kidneys are "set" to retain a definite percentage of sugar in the blood. The kidneys may be injured by the injection of the drug phloridzin. There is a fall in the level of blood sugar, and sugar appears in the urine for several hours. The amount of sugar in the blood falls below the normal. The mechanism of the process is undecided. Possibly the excretion of glucose is an active process, and not - simply the passive action of a dam to keep back a certain amount of sugar. In this case phloridzin might act by stimulating the exeretion of sugar by the kidneys. It is of interest in this connection that the drug causes marked degeneration of kidney 
epithelium. There is some reason to believe that phloridzin causes excretion of glucose in organs other than the kidneys.

A rather rare condition known as renal diabetes is similar to phloridzin diabetes in that sugar appears in the urine as a result of a deranged condition of the kidneys. In this disorder the level of blood sugar falls below normal, a fact which serves to differentiate it from diabetes mellitus. The kidneys pour out sugar into the urine even when blood sugar is at its normal level, thus reducing it in amount. Evidently renal diabetes is a disorder quite different in character from the ordinary type of diabetes.

Much speculation has been expended on the eause of diabetes in man and its possible relationships to one or other of the forms of experimental diabetes discussed, i.e., puncture diabetes, pancreatic diabetes or phloridzin diabetes. Obviously it is not analogous to the last form, for the disease is accompanied by inereased sugar content of the blood. Clinicians generally are of the opinion that it is closely allied to panereatic diabetes. Evidence, however, is not absolutely conclusive as yet, though it is generally believed that lesions in the pancreas are usually if not always the eause of the disorder.

In the study of diabetes much use has been made of the socalled assimilation limit for carbohydrates. A normal person can take by mouth 300-400 grams of glucose, or even more without the appearance of sugar in the urine. If sugar appears in the urine after taking 100 grams of glucose, the subject is considered to be diabetic. Woodyat has suggested a new term, - sugar tolerance, based on the amount of glucose which can be injected without causing glycosuria (sugar in the urine). $\mathrm{He}$ reports that in the normal person 0.8-0.9 grams glucose per kilo of body weight per hour can be used by the body. In a diabetic, of course, the amount would be smaller than this, and if the above mentioned amount is injected, sugar appears in the urine.

A review of the foregoing discussion will emphasize the fact that the internal factors regulating carbohydrate metabolism in the body include a center in the medulla, presiding over the 
conversion of liver glycogen into blood sugar, the internal secretion of the suprarenals, operative in a similar way, the internal secretion of the pancreas, without which sugar cannot be burned by the cells, and the nature of the kidney, which regulates the level of sugar in the blood.

Various forms of temporary glycosuria are known. Thus, after excessive exercise, during great agitation or mental strain such as often is experienced by students taking a difficult examination (emotional glycosuria), or after taking excessive amount of simple carbohydrates (alimentary glycosuria) sugar may appear in the urine. In the case of the first two conditions, the glycosuria is believed to depend on a production of adrenaline, which is known to be secreted at times of great exertion or emotional stress, a logical process, since at such times the muscles are apt to need an increased supply of fuel for use in possible pursuit, flight, or combat.

An important phase of carbohydrate metabolism is concerned with the ultimate fate of the glucose which is burned as fuel. How and where does the burning or oxidizing take place, and how is it controlled? There is still much uncertain ground in this field, though much progress has been made. Various methods have been employed to throw light on the problem. Interesting results have developed from a study of the Respiratory Quotient. This term is used for the ratio between the amount of carbon dioxide excreted in the respired air, and the amount of oxygen consumed, and is indicated as $\frac{\mathrm{CO}_{2}}{\mathrm{O}_{2}}$. If glucose is oxidized to $\mathrm{CO}_{2}$ and water a certain amount of oxygen is consumed.

$$
\mathrm{C}_{6} \mathrm{H}_{12} \mathrm{O}_{6}+6 \mathrm{O}_{2} \rightarrow 6 \mathrm{CO}_{2}+6 \mathrm{H}_{2} \mathrm{O} \text {. }
$$

For every molecule of $\mathrm{CO}_{2}$ produced, one molecule of $\mathrm{O}_{2}$ is used up. There already is enough $\mathrm{O}$ in the carbohydrate to take care of the hydrogen present. Thus the volume of $\mathrm{O}_{2}$ consumed equals the volume of $\mathrm{CO}_{2}$ produced, and $\frac{\mathrm{CO}_{2}}{\mathrm{O}_{2}}=1$. This will be true in the body as well as elsewhere provided the oxidation 
of the carbohydrate to $\mathrm{CO}_{2}$ and $\mathrm{H}_{2} \mathrm{O}$ is complete. If a fatty acid is burned in the same way, the respiratory quotient is less than one. It does not contain sufficient oxygen to take care of even the hydrogen. Thus part of the oxygen consumed is excreted as water, and in the expression $\frac{\mathrm{CO}_{2}}{\mathrm{O}_{2}}$, the denominator is larger, and the ratio is less than one (about 0.7 ). In the case of amino acids (from the proteins) the value lies between those for carbohydrates and fats (about 0.8). By actual measurement of the amount of $\mathrm{CO}_{2}$ excreted by an animal, and the amount of $\mathrm{O}_{2}$ consumed it is possible to draw conclusions as to the kind of material which the body is burning.

Further evidence of the fate and history of glucose in the body has been obtained by a study of the various experimental glycosurias described above.

Although much labor has been expended to clear up the exact mechanism of the burning of glucose in the muscles, very little is known of the steps in the process. We are sure that glucose serves as fuel for the muscles from evidence of various sorts but only in the presence of a substance produced in the pancreas. It has been suggested that glyceric aldehyde $\mathrm{COH}-$ $\mathrm{CHOH}-\mathrm{CH}_{2} \mathrm{OH}$ is an intermediate stage in the process of burning sugar, and that this is converted next into alcohol $\mathrm{CH}_{3}$ $\mathrm{CH}_{2} \mathrm{OH}$ and $\mathrm{CO}_{2}$, but this has not been proven. The diabetic is unable to use glucose, but the exact reason for the failure of the tissues to use sugar is unknown. It is probable that in some cases at least, the necessary internal secretion of the pancreas is wanting, but this only brings us a step nearer the solution without actually furnishing it. The diabetic is still capable of performing oxidations, for many substances other than glucose still are oxidized with ease. It has been suggested that the difficulty is in the first attack of cleavage on the sugar molecule, but there is some evidence which does not bear out this idea. We shall have to await the final solution of the problem.

Sources of Glycogen.-The study of the sources from which glycogen can be built up in the body has been greatly facilitated 
by the knowledge of the experimental glyeosurias. It is of interest to know what substances are glycogen formers in the body. One method of study is to render an animal as nearly as possible glycogen free, and then to feed the substance to be investigated. The animal then may be killed and the glyeogen eontent of liver and muscles estimated. If glyeogen is present in quantity it either will have been formed from the material fed, or indirectly, if this food has "spared" or been burned in place of some other glyeogen former. A second method is to render the animal diabetic by puneture, phloridzin or other means. A substance then may be fed, and the amount of sugar in the urine estimated. An increase will indicate that the substance fed is a glycogen or rather glucose former, provided the possible origin of the excess glucose from body constituents is exeluded. To render an animal glycogen free, it may be made to fast for some time, to do work, it may be subjected to cold, or thrown into convulsions by giving strychnine. A combination of methods usually gives more satisfactory results than any single method. A method of study depending upon quite different technique may be used. The liver may be exeised, and kept supplied with a circulating medium, either blood or some other fluid. The substance to be studied then may be introduced into the circulating fluid, and the glycogen content of the liver later determined.

It has been found, as might be expected, that glucose forms glycogen. Any substance which will form glucose in the body, thus also will be a possible glycogen former. Fructose, also, and to some extent galactose form glyeogen. On hydrolysis, this glyeogen is converted into glucose and not into the sugar from which it was produced. This is an interesting fact, as it is an example of a conversion of one monosaccharide into another in the body. Naturally all earbohydrates which are digested to monosaccharides in the alimentary tract thus will be sources of glyeogen. It has been shown, however, that there are sourees of glyeogen other than the carbohydrates.

If an animal is made diabetic by removal of the pancreas or in some other way, glueose appears in the urine. It continues to 
be excreted when the glycogen supplies of the body have been exhausted. If protein is fed to such an animal, the amount of glucose in the urine increases. There is a certain parallelism between the amount of glucose (dextrose) excreted and the amount of nitrogen in the urine. This is known as the D:N ratio. All the protein is not converted into glucose. Portions of some of the amino acids are destroyed or converted into other substances. Sixty grams of glucose is considered the maximum amount which the body can produce from 100 grams of protein (this contains ca. 16 grams nitrogen). A ratio of $\mathrm{D}: \mathrm{N}=60: 16$ or about $3.7: 1$ would indicate that none of the glucose produced from protein was being burned by the body, in other words a complete failure on the part of the tissues to burn glucose. 3.7 is thus known as the fatal ratio. It must be observed, however, when the animal is on a carbohydrate free diet, as otherwise the ratio may, of course, be still higher. By observing the $\mathrm{D}-\mathrm{N}$ ratio when various amino acids were fed, it has been shown beyond doubt that some amino acids are converted completely into glucose in the body, some others only partially. There is thus no question of the formation of glucose (and glycogen) from the carbon chain of the amino acids, and thus indirectly from the proteins.

Very little glycogen is formed from fat. The glycerine portion may be converted into glucose but the fatty acids do not appear to be converted into glucose.

Metabolism of Fats.-The fats are digested in the alimentary tract and absorbed in the form of fatty acids and soaps. In this process the bile salts play an important rôle. In the cells of the villi, however, a re-synthesis of neutral fat takes place, and at least most of the fatty acids and glycerine are recombined, and poured into the blood stream by way of the thoracic duct. Fats are stored away in a variety of places,-subcutaneously, in the intramuscular spaces, around the abdominal viscera and elsewhere. There is practically no limit to the amount which may be laid away. This is in sharp contrast to the non-storage of excess protein, and to the limited glycogen reserves. Fat is 
a reserve fuel, and it also protects the body from loss of heat, like a subcutaneous blanket, for it is a poor conductor.

If an animal is fed excessive amounts of carbohydrate food, it will lay on reserves of fat. There is evidence thus that carbohydrates may be converted into fat in the body, although it seems that the reverse process does not take place, at least it is very questionable. Since amino acids may be converted into carbohydrates, they also may be fat formers.

Recent work has made it evident that there is considerable difference in the character of the fat-like substances in different tissues. The fats of the great inert fat deposits are mostly true neutral fat, whereas the "fats" in the active tissues are probably largely in some other form, perhaps lecithin or allied substances. On hydrolysis, these yield a much lower percentage of fatty acids. The "fats" of the liver are intermediate between depot and tissue fats. Blood "fat" probably is largely lecithin or some similar substance. It is likely that the depot fats, when mobilized, are converted in the liver into lecithin or something similar, and also to a certain extent the acids converted into unsaturated acids. This process may be regarded as preparatory to the use of the fats by the cells.

Light has been thrown upon the mechanism of fat oxidation in the body in various ways. Ordinarily the fatty acids are burned completely to $\mathrm{CO}_{2}$ and $\mathrm{H}_{2} \mathrm{O}$. By introducing into the organism compounds in which a fatty acid side chain is attached to a benzene ring, the last step in this destruction of the side chain is prevented and the nature of the resulting substance may be studied. Such compounds in which the side chain has an even number of carbon atoms are oxidized, and the side chain destoyed with the exception of two carbon atoms. From phenylbutyric acid, phenyl acetic is produced. This substance is conjugated with glycocoll and excreted as phenyl aceturic acid, a compound of phenyl acetic and glycocoll analogous to hippuric acid (q.v.). If the original side chain contains an uneven number of carbon atoms, the side chain is oxidized away, and benzoic acid remains, which is conjugated with glycocoll and 
excreted as hippuric acid. From these facts it is evident that the side chains are oxidized so that not one carbon atom, but two are removed at each step.

Thus it is believed that the fatty acids from the fats also are oxidized two carbon atoms at a time, the final products normally being converted completely into carbon dioxide and water. The acetoacetic acid $\mathrm{CH}_{3}-\mathrm{CO}-\mathrm{CH}_{2}-\mathrm{COOH}, \beta$-oxybutyric $\mathrm{CH}_{3}-$ $\mathrm{CHOH}-\mathrm{CH}_{2}-\mathrm{COOH}$ and acetone which appear in the urine in the advanced stages of diabetes, when the organism is so severely affected that its powers of oxidation are impaired are believed to be largely a product of the incomplete oxidation of fatty acids, though acetoacetic has been shown to arise also from certain of the amino acids.

Interesting work has recently been published by Shaffer on the conditions governing the appearance of acetone bodies in the urine. It has become evident that the proper burning of fatty acids in the body is influenced by the presence of glucose. Since fatty acids are a source of the acetone bodies, as are also certain of the amino acids, Shaffer calls these compounds "ketogenetic" (Symbol K). Glucose, both that from the food carbohydrates and from amino acids or other sources he calls "antiketogenetic" (Symbol A). When the value $\frac{K}{A}=2$ or more, acetone bodies appear in the urine. A method has been devised on this basis whereby it is possible to calculate the amount of carbohydrate it is necessary to supply to a diabetic to prevent the excretion of acetone bodies in the urine.

Metabolism of Inorganic Material.-The metabolism of inorganic material assumes an aspect somewhat different from that of the organic substances, for the reason that inorganic substances are not disintegrated or "burned" in the organism, and thus are not sources of energy. There is, of course, more or less interchange of radicles, and to a certain extent inorganic substances are built into compounds of organic nature. But quite aside from this, inorganic materials play a very important part in metabolism. Numerous chemical reactions in the body are 
controlled, or influenced by salts. The irritability of muscle and nerve is greatly affected by the kind and amount of salts present. The elotting of blood and of milk both are dependent upon the presence of calcium. The general osmotic equilibrium of tissues and fluids depends in large measure upon salts. Even the development of the unfertilized eggs of some animals may be stimulated by certain salts. Evidently the inorganic materials of the body have far more extended importance than merely to form an inert framework to support and protect the soft organs and tissues.

Osborne and Mendel, in 1918 fed rats on diets which were adequate in every respect except their content of inorganic materials. Various salts were added to this diet. It was found that the omission of chloride, phosphorus, sodium, potassium and magnesium caused the animals to stop growing. Evidently certain salts are necessary for normal growth. It is also well known that a calcium-free diet results in faulty bone formation. Hart, McCollum and Humphrey fed a cow on a diet deficient in calcium. In a little over three months she lost $5.5 \mathrm{lbs}$. of calcium from her own body. It is quite evident that an adequate supply of inorganic materials must be included in the diet. The ordinary mixed diet of the average adult usually contains these substances in sufficient quantity and diversity. Certain conditions involving faulty bone formation, such as rickets, are not necessarily due to shortage of inorganic materials in the food, however, but to deranged metabolic conditions; which result in failure on the part of the organism properly to use its available supplies (see discussion of vitamines).

Energy Exchange.-A very important phase of metabolism is concerned with the body's energy requirements. Very amusing ideas on this subject prevailed up to a century and a half ago. The foundations of present day knowledge were laid by the great Frenchman, Lavoisier, who demonstrated that there was something in common between the burning of a candle and the breathing of an animal. Mice died and candles went out if placed under a bell jar, and either one shortened the period of 
a subsequent mouse or candle. He decided that the burning of the candle consisted in a combining of the carbon of the candle with a substance in the air which he called "oxygen," and that in animals a similar process took place, producing the heat of the body. By measuring the amount of ice melted in a given time by the heat from an animal, and comparing the result with the amount of carbon dioxide produced by the animal in a period of similar length, he obtained results which indicated that at least $96 \%$ of the heat produced by the animal could be accounted for by the oxidation of carbon to $\mathrm{CO}_{2}$. He further showed that oxygen also was used up in combining with hydrogen to form water. He also arrived at the result that more heat was produced when the animal was subjected to cold than otherwise, and that digestion and work increased the heat output. Unfortunately, Lavoisier lost his life on the guillotine during the Reign of Terror, and his brilliant work was left unfinished.

The way had been opened, however, and other workers took up the problems which the brilliant Lavoisier had opened. The methods and ideas of Lavoisier were extended and improved. Dulong and Depretz, Regnault and Reiset, Rubner and others abroad, and Atwater, Benedict, Lusk, du Bois and others in this country have investigated and solved many of the problems connected with energy balance and energy requirements. The work of Atwater in this country has been of particular service from the point of technique development. Atwater, in conjunction with Rosa constructed an apparatus known as a calorimeter. This contrivance consists of an insulated chamber large enough for a man. The walls are so constructed that no heat is lost through them. The heat produced by the occupant is carried off by water circulating through a cooling coil. The flow of water and its temperature at entering and leaving the chamber can be accurately measured, and thus the amount of heat carried off from the chamber computed. A circulation of air is provided, and from this, the moisture evaporated from the subject is extracted and measured, and the heat consumed in its vaporization calculated. A third factor in the heat measure- 
ment consists in observing any change in the temperature of the patient or the apparatus. The sum of these three factors will give the total heat given off by the subject.

The circulating air is in a closed system. The carbon dioxide is removed by passing the air through an absorption apparatus, and its amount can be determined by weighing. Oxygen is supplied from an oxygen retort, so that the amount used by the subject ean be determined accurately by the loss of weight of the retort, considered in connection with any change in the composition of the circulating air.

By means of this calorimeter, accurate measurements may be made of the heat produced in the body, and the amount of carbon dioxide and water produced. Results of the most extraordinary accuracy have been obtained by Atwater, Benedict, Lusk, du Bois, and others, who have worked with apparatus constructed on this general principle.

One of the most important and far reaching results obtained in this way is that the law of conservation of energy holds for the animal body. The animal body can neither create nor destroy energy. In a series of experiments representing an exchange of over 590,000 calories, the difference between the theoretical calculation and the amount actually measured was only 50.1 calories, an error of only about $0.1 \%$. Thus an animal works on the same principle as an engine, a candle or an alcohol flame. The heat which it produces comes from the oxidation of organic substances, either those of the food, or those of the body tissues.

In calculating the amount of heat which will be produced in the body by a given foodstuff, it must be burne in mind that only the portion of the food which is absorbed will be available for burning in the cells, and also that if the food is not completely burned to carbon dioxide and water, this fact must be taken into account. The carbohydrates ordinarily are completely burned to $\mathrm{CO}_{2}$ and $\mathrm{H}_{2} \mathrm{O}$, as are also the fats. The proteins, however, are not completely oxidized, for uric acid, urea, and other substances of protein origin are excreted in the urine. 
The figures usually used for the average energy value of the three foodstuffs are carbohydrates 4.1 large calories per gram, proteins 4.1 and fats 9.3 . It will be seen that the fats yield by far the most heat per gram. (It will be recalled that a large calorie is the amount of heat required to raise 1 kilo of water through $1^{\circ} \mathrm{C}$. of temperature,-usually from $15^{\circ}$ to $16^{\circ} \mathrm{C}$. or from $0^{\circ}$ to $1^{\circ}$ C.)

Accurate and extended studies have been made of the amount of heat produced (and thus the amount of fuel required) by individuals in health and in various diseases, at rest and during exercise, waking and sleeping, during periods of mental ease and of great mental exertion, at different ages from infancy to old age, and in the two sexes. It will be possible here only to summarize briefly some of these important findings.

First, the different foodstuffs are isodynamic in the body, that is, the body can employ fats, carbohydrates or proteins as fuel interchangeably, and with no loss of energy,-each foodstuff furnishes its total theoretical amount of energy when it is oxidized in the body.

The total amount of energy required by an individual varies with his age, state of health, body weight, sex, and degree of body activity. In general, the energy exchange is higher in small animals than in larger ones, for there is more surface per unit of weight in small animals, and thus the loss of heat is greater. Metabolism in children is higher than in adults, partly for the foregoing reason, and possibly also because there is greater tissue activity. In newborn infants during the first few days of life, metabolism is low. In a fat man the energy exchange per kilo body weight is lower than in a thin man, as the excess weight is due to inert fat deposits which take little part in active metabolism. Metabolism is lower per kilo body weight in women than in men.

Increase in body activity, of course, increases the energy exchange. More fuel is burned, and more heat produced. The average city adult man requires from 2,500 to 3,000 calories per day. If such a person remains in bed and inactive, his energy 
requirement sinks to perhaps 1,700 calories. Physical exercise and exposure to cold greatly increase the energy requirement, which may run up to 4,000 or even 5,000 calories. The extreme limit recorded is the case of an endurance bicycle rider who used up 10,000 calories in a day.

An interesting fact is that the mere taking of food will materially increase the heat production in the body. It was suggested that this was due to the work of the digestive glands and organs, but this has been shown to be incorrect; the taking of salts which cause intense muscular activity of the intestine resulted in no such increase. This effect of food substances is spoken of as the Specifie Dynamic action of the foods. Proteins cause the greatest rise in heat production. The effect in the case of proteins is believed to be due to a stimulation of the cells of the tissues to greater activity, the stimulation being produced by the decomposition fragments of protein in the blood. In the case of the earbohydrates and probably also of the fats, the effect is believed to be due to the "mass action" of the fragments of decomposition and not to a direct stimulating action. Since proteins produce the greatest increase in heat production, they should be eaten sparingly in hot weather.

Curiously enough, increased mental activity does not increase the heat produced by the body. This might be interpreted to mean that mental activity uses up no fuel, or an inappreciable amount, but it is more nearly accurate to state that great mental activity such as studying for an examination produces no more heat than the mental activity of our ordinary and most indolent mental processes.

During sleep metabolism is low, but this is due probably to lessened body activity rather than to any inherent differences of metabolism.

The variations of heat production in disease have been carefully studied by Benedict, Lusk, du Bois and others. In typhoid and exopthalmic goitre there is a great increase. In diabetes the increase is slight.

As a basis for comparison, the heat production of the body at 
minimum activity is taken as a standard. This is called the Basal Metabolism, and is taken as the amount of heat produced by a person lying quietly in bed, protected from extremes of temperature, and having taken no food for 12-15 hours. There has been much discussion as to whether this should be referred to units of body weight or body surface. Apparently it is absolutely proportional to neither of these standards, but perhaps to the "mass of active protoplasm" in the subject. This it is of course impossible to determine experimentally. It has been found however, that the heat production is very nearly proportional to body surface, which easily can be calculated from a formula devised by $\mathrm{Du}$ Bois. Under basal metabolism conditions, the normal adult produces very close to 40 calories per square meter surface per hour. A deviation of $10-15 \%$ from this standard is still considered normal. Certain diseases are accompanied by marked deviation from this value. In exophthalmic goiter the heat production may run $50-75 \%$ above this normal, and in no other disease is it so high. In myxedema, and cretinism, where the thyroid activity is subnormal, the basal metabolism falls to a very low level. This fact is of great value in diagnosis, and in following the effect of treatment.

Basal metabolism may be determined not only by direct measurement of heat eliminated, which involves a costly and elaborate calorimeter, but also by the method of "Indirect Calorimetry." By the measurement of the oxygen consumed and $\mathrm{CO}_{2}$ exhaled by a patient in a given time it is possible to calculate the respiratory quotient. This, when considered with the amount of nitrogen in the urine, coming of course from protein destruction, gives all data necessary to calculate just how much fat, carbohydrate and protein have been burned in the body. Since we know the number of calories produced in the oxidation of 1 gram of each of these substances, it is possible to calculate the total heat production and thus the basal metabolism.

Apparatus for Indirect Calorimetry is within the reach, financially speaking, of every clinic, and its use as an aid to diagnosis has become very widespread. 
Utilization of Alcohol by the Body.-A problem of the greatest importance has been to determine the position of alcohol in metabolism. If burned in a calorimeter, alcohol yields over 7 calories per gram, and thus has a very high fuel value. A long and careful study of the effects of alcohol on the body has been undertaken by Benedict. In the body, small amounts of alcohol undoubtedly are burned as fuel by the tissues. The effects of alcohol are those of a depressant rather than of a stimulant, apparent stimulation being in reality a depression of inhibiting influences. Although alcohol undoubtedly is burned as fuel by the body, still it is a generally accepted fact that its poisonous effect on the cells more than counterbalances any beneficial effect resulting from its high fucl value.

Starvation.-If an animal is deprived of food it will live for some time, drawing upon its own tissues for the essential materials for fuel and body maintenance. The length of time an animal will survive without food varies greatly with the size and condition of the animal, in general a large or a fat animal will survive longer. It depends also much on external conditions. If exposed to cold or great exertions as in winter, in shipwreck, etc., life may be lost in a few hours. Otherwise, fasting may be continued for even a month or longer in the case of a man, without death ensuing. Children die much sooner, usually in four or five days. Recently the case of a dog was reported in which the animal survived 117 days of fast. If water is withheld as well as food, death occurs in a few days' time.

The study of metabolism during fasting furnishes interesting information about the chemical processes going on in the body. Benedict has published an exhaustive study of a man fasting 31 days.

During fasting there is of course continuous loss of body weight. Reserve stores of glycogen and fat are called upon, but there also is a continuous excretion of nitrogenous material in the urine, showing that some protein is continually broken down. At an earlier point we have noted the appearance of creatine in 
starvation, replacing a portion of the normal creatinine. A most interesting fact is that all tissues and organs do not lose weight in like amount as starvation proceeds. Organs of vital importance such as the heart, the brain and nerves are preserved practically without loss of weight, whereas the skeletal muscles, the liver (loses glycogen) and adipose tissue lose a very considerable portion of their weight. The body tries to save the most vitally important organs, and does so at the expense of less indispensable tissues, which are called upon to furnish fuel and undoubtedly also repair material for the vital parts.

The nitrogen excretion of a fasting animal gradually decreases as time goes on, probably as a result of the decreased amount of protein tissue in the body. Shortly before the death of the animal the nitrogen excretion rises sharply. This is known as the pre-mortal rise. It appears as if the organism gives up the struggle to protect its tissues from destruction and that the overtaxed organism begins to give way. After a prolonged fast, the total nitrogen excretion in man falls to the neighborhood of about 2 grams per day. This appears to be the minimum, and has been taken by some to represent the actual necessary wear and tear of the protein tissues of the body. Possibly, however, it is necessary for the body to break down the protein which this represents in order to obtain some particular amino acid or acids needed in the synthesis of some absolutely essential product of internal secretion.

Several cases are on record, and the author himself has known individuals who have derived great benefit from an occasional wisely timed fast. The digestive and excretory organs are given a rest, the tissues are cleared of all unnecessary stored up material, and the body undergoes a thorough housecleaning.

Unknown Food Constituents._-Vitamins.-Until recently it was believed that if an individual were supplied with enough protein, the necessary salts, and amounts of carbohydrates and fats sufficient to make up the total of energy required, that his diet was adequately regulated. Recent developments have shown that this list is incomplete, and that certain substances 
hitherto undreamed of, are absolutely essential to the health and even the life of the individual. The chemical nature of these substances is still unknown, but it appears that there probably are at least four and possibly more of them.

The discovery of the existence of these substances was intimately connected with a disease known as beri-beri prevalent in oriental countries where the natives live on a diet consisting mainly of polished rice. On such a diet, extensive disturbances of the nervous system develop, resulting in weakness, paralysis and ultimate death. Feeding the outside layer of rice, ordinarily removed in polishing, causes immediate relief and speedy recovery. A substance evidently is present in the outer layer of rice, without which an animal on a polished rice diet develops beri-beri. Later work has shown the substance to be present mainly in the embryo. A similar condition appears in chickens on a rice diet, and extensive studies have been carried out to ascertain the nature of the curative substance and of the disease. Funk has called the unknown material "vitamine," a name to which there are some objections, and believes that the substance contains nitrogen.

The investigation of these unknown, but essential dietary constituents is made difficult by the fact that they are present in the foods and are required by the body in extremely minute amounts, a few milligrams being sufficient to supply all the body's daily needs.

Just why the body needs these materials and the functions which they perform are matters as yet obscure. Possibly they stimulate or regulate the functioning of certain tissues or cells, possibly they are used as materials for the construction of certain products of internal secretion which are required in the processes of metabolism. The riddle is still unsolved, but nevertheless, we are closer to its solution than were our ancestors, who did not dream of the existence of these vitally important compounds.

Since the earlier observations were made, much interest has centered on diseases which appear to be brought about by 
deficiencies in the diet, and the term "vitamin," now often spelled without the final " $e$," has become a household word. It is possible very briefly to summarize the enormous amount of work which has been done in this field in the last ten years.

At present nothing is known of the chemical constitution of the vitamins. At least four are believed to exist, and since they are of unknown structure they have been given the provisional designations A, B, C, and D. Absence of any one of these substances from the diet will eause serious disorders even to the point of death. In the case of an animal suffering from a lack of one of the vitamins, remarkable recovery may be brought about by feeding extremely small amounts of the missing substance, either in the form of an extract or a food containing the vitamin. The following brief summary gives the essential facts which are known at present.

Vitamin A.-A shortage of $A$ results in failure to grow in young animals, and in a disease of the eyes, xerophthalmia, in which the eyes are very subject to infection, and which may result in blindness. $A$ is found in butterfat and milk, in which it is present in particularly large amounts if the cow has been fed on green fodder, in smaller amounts if the animal has been stable fed. It is found in egg yolk, the germ of cereals, and green leaves for example eabbage and lettuce, and also in large amounts in cod liver oil. It may be stored in the body to some extent. It is soluble in fats, ether, alcohol-ether and some other solvents. It is fairly stable to heat, quite so in acid and less so in alkaline solution. It is stable to drying, but is destroyed by hydrogenation. By reason of its distribution and stability it is not likely to be lacking in the diet, but dairy products and green leaves should be included to secure an adequate supply.

Vitamin B.-A shortage of $B$ results in failure to grow, in the young, and in adults leads to the disease known as beri-beri, mentioned above. More recent work has shown that $B$ has a marked effect in stimulating the appetite, and much evidence points to the conclusion that it has some connection with the sex interests and functions. Rats on a diet deficient in $B$ become 
sterile. A lack of this substance also leads to marked disturbance of the digestive system. Some reports indicate that $B$ is concerned in oxidation in the cells, and perhaps in the metabolism in the cell nucleus.

$B$ is found in largest amounts in yeast, but also in green leaves, spinach, lettuce, asparagus, celery, beans, peas, milk, including milk powder, the embryo of grains, and eggs. The amount in dairy products varies with the amount in the food of the cow, as evidently the animal does not synthesize it. It is soluble in water, and probably is not stored in the body. It is not destroyed by drying, is stable at $100^{\circ} \mathrm{C}$. in neutral or acid solution, but is destroyed by heating in alkaline solution. Commercial canning or sterilizing processes are likely to destroy it, if temperatures above $100^{\circ}$ are used. On account of its wide distribution and its comparative stability it is not likely to be lacking in the diet.

Vitamin C.-A disease which ean be cured by $C$ is scurvy, which long was the terror of seafaring men. A shortage of $C$ also affects the teeth. It has been reported also that $C$ is concerned in the production of adrenaline and in normal metabolism. If is found in oranges, lemons, cabbage, lettuce, and tomatoes ir. largest amounts, also in spinach, onions, fresh peas, and in small amounts in potatoes. Milk contains some, but most prepared milks but little $C$. Much depends on the food of the cow, as indicated for $A$, and on the method of preparing the milk powder. Germinating seeds contain much. By allowing seeds to sprout the amount of $C$ is greatly increased. Drying destroys $C$ except in acid solution, such as orange, lemon or tomato juice. It is soluble in water and is more stable in acid than in alkaline solution. In the absence of oxygen it is stable up to $100^{\circ} \mathrm{C}$. (Dutcher). C, by reason of its more limited distribution, its susceptibility to drying and to high temperatures is more likely to be deficient in an ordinary diet than $A$ or $B$. Green leafy vegetables, oranges, lemons, cabbage or tomatoes should be included in the diet to secure an adequate supply.

Vitamin $D$.- - It is not altogether certain that vitamin $D$ exists, 
but most recent evidence indicates that cod liver oil contains some substance other than $A$, the taking of which makes it possible for an animal to make better use of the calcium in his food than without this substance. This substance, $D$, is curative for rickets. But rickets also may be relieved or eured by giving phosphates, or exposure to sunlight. The case of rickets is thus by no means clear.

Other disolders have been reported as deficiency diseases, but the evidence is not conclusive. Doubtless time will bring to light other vitamins, and solve many of the problems in this field which still are unsettled.

Body Temperature.-In warm blooded animals body temperature must be maintained at a constant level, regardless of external conditions. Fuel is burned in the body, and heat produced. In lower animals, a fall in the external temperature seems directly to stimulate increased heat production in the body. In man such a direct result appears to be wanting, but the same end is accomplished indirectly by stimulation of shivering or increased voluntary activity. More fuel is burned, and more heat produced.

It appears that the heat situation in the body is presided over by a center, possibly two centers in the medulla. One of these centers presides over heat production, the other over a balance between heat production and heat loss. Heat loss is accomplished by increased excretion of sweat, which evaporates from the skin and thus removes excess heat. Sweating may occur even in cold weather during vigorous exercise, when the heat production is greatly increased. If evaporation from the surface of the body is prevented, either by coating the body with some substance, or if the surrounding air is saturated with moisture, great discomfort is experienced. The temperature of the body rises, and death will result if the condition is not relieved.

Influence of Organs of Internal Secretion.-The metabolism of the body as a whole, and of specific organs or tissues is profoundly influenced by products poured out into the blood stream 
by certain glands and other tissues. Since these products are delivered into the blood stream they are called internal secretions. We already have considered the internal secretion of the adrenals, adrenaline, in its effect upon the control of liver glycogen, the internal secretion of the pancreas and its importance for the utilization of sugar by the muscles, the hormones secreted by the stomach and intestinal walls, and their importance in inducing the flow of digestive juices. To this list many more examples might be added. Thus the thyroid gland is known to have a profound influence on general metabolism. If the gland atrophies, or is removed in a young individual, growth is retarded and dwarfism results. Mental development also is checked. The condition is known as cretinism, and it may be improved by feeding thyroid. In adults, removal or atrophy of the gland results in thickening of the skin, and coarseness of the hair, the temperature is below normal and general depression of metabolism is observed. If the thyroid is too active or too large, goitre results, with a general quickening of metabolism and increased nervous irritability. These and other facts are interpreted to mean that the thyroid pours out a substance into the blood which has a profound effect on general metabolism, and influences the metabolism of the bones, the nerves and the skin. This substance undoubtedly contains iodine. It is being studied by Kendall. The parathyroids undoubtedly also have important functions, but they are not so well understood. Careful removal of these organs causes symptoms of poisoning, but the cause is still obscure.

In addition to the action of adrenaline above mentioned, this secretion of the suprarenal glands increases vasomotor tone and has other less understood functions. Even if adrenaline is injected into an animal from which the suprarenals have been removed, the animal dies, but the cause of its death is unknown.

The sexual glands also markedly influence metabolism. Removal of the testes in immature males, a process known as castration, results in the failure of the secondary sexual character- 
istics to appear. This effect is considered to be due to lack of substances poured out into the blood stream from these organs.

The anterior lobe of the pituitary body is known to have a marked influence on growth. If it is removed from a young animal, the animal fails to grow, and retains its infant characteristics such as immature intelligence, infantile sex organs, etc. If there is an excessive development of this portion of the hypophysis, gigantism results. The posterior lobe seems to have no such function. The only symptoms reported after its removal are an increased sexual desire, and an increased flow of urine.

The thymus and pineal glands probably have functions in connection with general metabolism, but these functions as yet are obscure. 


\section{PART II}

\section{LABORATORY WORK}

Directions for laboratory work for each chapter except $\mathrm{X}$ and XII are appended. Lists of apparatus and materials needed have been included as an aid to the teacher. Directions for making up the necessary quantitative solutions will be found at the end of the volume.

\section{MATERIALS}

The following materials should be available:

1. Cleaning fluid-a saturated solution of potassium bichromate in concentrated sulphuric acid.

2. Soap solution.

3. Sand or damp sawdust, placed for instant use to extinguish small fires.

4. A blanket or woolen rug is a valuable aid in case a student's clothing catches fire.

5. A supply of sodium bicarbonate solution should be kept on hand for use if a student gets acid in his eyes. Wash under running water and pour on sodium bicarbonate solution.

6. Boric acid solution should be kept for use if a student gets alkali in his eyes.

The following list includes the apparatus desirable for the equipment of the desk of each student. It is supplemented occasionally by articles obtained temporarily from the storeroom.

4 Beakers, assorted sizes.

1 Doz. test tubes.

1 Erlenmeyer flask, 250 c.c.

1 Erlenmeyer flask, 500 c.c.

2 Florentine flasks, 500 c.c.

1 Florentine flask, 1000 c.c.

1 Funnel, large.

1 Funnel, small.

1 Graduate, 10 c.c.
1 Graduate, 100 c.c.

11 c.c. pipette, graduated in $1 / 100$ c.c.

15 c.c. pipette.

110 c.c. pipette.

120 c.c. pipette.

125 c.c. pipette.

2 Watch glasses.

1 Wash bottle.

2 Kjeldahl flasks, 500 c.c. 
1 Woulff bottle, $\mathrm{CaCl}_{2}$ tube and Several sheets hard filter paper. glass pearls.

1 Kjeldahl condenser bulb and connections.

1 Burette and clamp.

1 Large glass stoppered bottle.

1 Stirring rod.

1 Porcelain casserole.

1 Porcelain crucible and cover.

1 Small evaporating dish.

1 Large evaporating dish.

1 Thermometer.

$1 / 2$ Pkg. small filter paper.

$1 / 2 \mathrm{Pkg}$. large filter paper.

2 Bottles litmus paper.

1 Test tube holder.

1 Test tube brush.

1 Test tube rack.

1 Clay triangle.

1 Wire gauze.

1 Iron stand and ring.

1 Tripod with 2 rings.

1 Bunsen burner and tubing.

1 Box of matches.

1 Glass slide.

Key to locker.

Several small labels.

On each desk, or conveniently placed should be the following reagents :

1. Acid, acetic glacial.

2. Acid, acetic, $10 \%$.

3. Acid, hydrochloric, conc.

4. Acid, hydrochloric, $10 \%$.

5. Acid, nitric, conc.

6. Acid, nitric, $10 \%$.

7. Acid, sulphuric, conc.

8. Acid, sulphuric, $10 \%$.

9. Acid, picric, sat'd sol.

10. Alcohol, $95 \%$.

11. Ammonium hydroxide, $10 \%$.

12. Ammonium molybdate, $10 \%$.

13. Ammonium oxalate, $2 \%$.

14. Ammonium phosphate, $2 \%$.

15. Ammonium sulphocyanate, $2 \%$.

16. Barium chloride, $5 \%$.

17. Chloroform.
18. Copper sulphate, $5 \%$.

19. Ether.

20. Fehling, Sol. A.

21. Fehling, Sol. B.

22. Ferric chloride, $2 \%$.

23. Aqueous iodine, $1 \%$.

24. Mercuric chloride sat.

25. Millons reagent.

26. Potassium ferrocyanide, $2 \%$.

27. Silver nitrate, $2 \%$.

28. Sodium carbonate, $2 \%$.

29. Sat. sodium chloride.

30. Sodium chloride, $10 \%$.

31. Sodium hydroxide, sat.

32. Sodium hydroxide, $10 \%$.

33. Benedict's solution.

The following lists cover the special materials and apparatus needed for the work in the laboratory chapters:

SoLids.

Gelatin.

Dialyzing tubes.

Starch.

\section{Chapter I.}

Liquids.

Methyl orange, $1 \%$ aqueous.

Phenolphthalein, $1 \%$ alcoholic.

$0.1 \mathrm{~N}$ Oxalic acid.

Colloidal (dialyzed) iron.

Albumin solution. 


\section{Chapter II.}

Solids.

\section{LIQUIDS.}

Meat (hamburger steak) $1 \mathrm{lb}$.

Lead acetate sol. enough for 25 students.

Solid casein.

Soda lime.

Fusion mixture.

Bone ash.

Blood or blood serum-20 c.c. per man.

Potassium hydroxide, $25 \%$.

Potassium pyroantimonate sol.

Sodium cobaltinitrite sol.

Ground bone.

Bismuth subnitrate.

SoLIDS.

Dextrose.

Yeast-1 cake for 25 students.

Cane sugar.

Maltose.

Lactose.

Potatoes-1 for each student.

Starch.

Dextrin.

Orcin.

Phlorgluein.

Special Apparatus.

Microscope.

Polariscope and its accessories.

Fermentation tube.

Incubator.

Steam bath.

Knives for scraping potato.

Cheese cloth for straining.

Solids.

Lard.

Boric acid or $\mathrm{KHSO}_{4}$.

Fusion mixture.

\section{Chapter III.}

Solutions.

Dextrose, $2 \%$.

Levulose, $2 \%$.

Galactose, $1 \%$.

Arabinose (Hydrolize gum arabic with $\left.2 \% \mathrm{H}_{2} \mathrm{SO}_{4}\right)$.

Cane sugar, $2 \%$.

Maltose, $2 \%$.

Lactose, $2 \%$.

Glycogen.

Barfoeds sol.

Phenylhydrazine.

$15 \%$ alcoholic $\propto$ naphthol.

Tannic acid sol.

Amyl alcohol.

\section{Chapter IV.}

LIQUIDS.

Olive (or cottonseed) oil.

Soap solution.

Albumin solution.

Gum arabic sol.

Lymph (few drops if available).

Alcoholic NaOH, 75 c.c. per man.

Glycerine.

Calcium chloride sol.

Egg yolk or its ether extract.

Acetone, 40 c.c. per man.

Phenolphthalein, $1 \%$ alcoholic.

Milk.

$0.1 \mathrm{~N}$ alcoholic $\mathrm{NaOH}$ 


\section{Chapter V.}

Solids.

For Simple Proteins.

Casein.

Magnesium sulphate.

Ammonium sulphate.

Egg albumin.

Fibrin.

Meat (muscle).

Flour, 50 grams per man.

Horn.

Tendo Achilles.

Gelatine.

Ligamentum nuchæ.

For Conjugated Proteins.

Solid benzidene.

Carrots-raw and boiled.

Beef pancreas.

For Derived Proteins.

Witte's peptone.

Armour's peptone.

Barium carbonate.

Wool.

Charcoal.

Solid sodium acetate.

Congo paper.

Fusion mixture.

\section{Special Apparatus.}

Spectroscopes.

Flat sided cells for spectroscopic observations.
LIQUIDS.

For Simple Proteins.

Egg albumin sol.

Phenol, 1\% sol.

Glyoxylic acid sol.

Alcoholic $\propto$ naphthol, $15 \%$.

Potassium mercuric iodide sol., $2 \%$.

Acid phosphomolybdic, $2 \%$.

Acid phosphotungstic, $2 \%$.

Acid trichloracetic, $2 \%$.

Tannic acid.

Lead acetate sol.

Ammonium sulphate, sat.

Blood serum.

Egg white, undiluted, 5 c.c. per man.

Blood plasma, 20 c.c. per man.

Pepsin sol., $1 \%$ in $0.2 \%$, HCl.

Pancreatin sol., $1 \%$ aqueous.

Lime water, sat.

For Conjugated Proteins.

Defibrinated blood.

Stokes' reagent.

CO-hemoglobin, aerated.

Fresh potassium ferricyanide sol.

Defibrinated rat or guinea pig blood.

Hydrogen peroxide.

Guaiac.

Corpuscles, washed in physiol. saline.

Milk, 30 e.c.

Lead acetate sol.

Egg yolk extracted with ether.

Egg white diluted 1 to 10 .

Derived Proteins.

Egg white undiluted 10 c.c.

Meat digested with pancreatin.

Chapter VI.

For materials needed in this chapter, see the individual methods. 


\section{Chapter VII.}

Solids.

Paraffin.

Starch.

Freezing mixture (ice and salt).
LIQUIDS.

Phenolphthalein, 1\% alcoholic sol.

Methyl orange, $1 \%$ aqueous.

Cane sugar sol.

\section{Chapter VIII.}

\section{Solids.}

Pig's stomach.

Mett's tubes.

Thread.

Rennin tablets.

Capsules, 0.1 gram $\mathrm{CHI}_{3}$.

Capsules, 0.2 gram KI.

Capsules, 1.0 gram salol.

Starch paper.

Solid ammonium sulphate.

\section{SoLIDS.}

Mett's tubes.

Pancreatin powder.

Cane sugar.

Charcoal.

Powdered sulphur.

Gall stones.

\section{Chapter XI.}

\section{Solids.}

Sodium carbonate.

Barium carbonate.

Pure oxalic acid.

Pure sodium bicarbonate.

Potassium sulphate.

\section{Special Apparatus.}

Bottles for urine.

Large (2 liter) cylinders.

Urinometer.

Weighing bottles.

Quantitative balance.
LIQUIDS.

Glycerine.

Toepfer's reagent.

Guenzburg's reagent.

Congo red.

Phenol, 1\% sol.

Lactic acid, $1 \%$.

Milk.

N/10 alkali.

$\mathrm{CaCl}_{2}$.

\section{Chapter IX.}

\section{LiQuids.}

$1 \%$ aqueous pancreatin sol.

Milk, 5 c.c. per man.

Litmus sol.

Bile.
Liquids.

Urine, 2 or 3 liters per man.

Albumin urine.

Sugar urine.

Acetone urine.

Urine, a 24-hour specimen.

$5 \%$ Thymol in chloroform.

Magnesia mixture, 225 c.c. per man.

Potassium permanganate-about 8 or $10 \%$ sol.

Oxalic acid, about $5 \%$.

Bromine water.

Glycerol. 
Special Apparatus.

Fermentation tubes.

Polariscope.

Esbach tubes.

100 c.c. volumetric flask.

500 c.c. volumetric flask.

Duboseq colorimeter.

Distilling apparatus.

Cylinders, bottles, Folin absorption tubes for ammonia and urea determinations.

Compressed air (or a water pump).

\section{LIQUIDS.}

Apparatus for producing $\mathrm{H}_{2} \mathrm{~S}$.

Sodium nitroprusside sol. (fresh).

Methyl orange, $1 \%$ aqueous.

Calcium chloride sol.

Lead acetate sol.

Alizarine red, $1 \%$ aqueous.

$1 \%$ Potassium dihydrogen phosphate, 30 c.c. per man.

Neutralized potassium oxalate sol. (saturated).

Sodium hydroxide, saturated.

Kerosene.

Formalin, diluted, 1-4 and neutralized with $\mathrm{N} / 10 \mathrm{NaOH}$ (phenolphthalein).

Folin-Schaffer reagent.

10\% Ammonium sulphate.

$\mathrm{N} / 20$ Potassium permanganate.

N/2 Potassium bichromate.

Standard silver nitrate.

Standard ammonium sulphocyanate.

Ferric ammonium sulphate, sat'd (iron alum).

Standard barium chloride.

Special sodium acetate.

Standard uranium acetate.

Esbach's reagent.

Quantitative, Fehling's.

Benedict's sol.

For materials needed in Folin's microchemical methods for uric acid, urea and creatinine see the individual methods. 


\section{GENERAL LABORATORY INSTRUCTIONS}

Perform all experiments with eare. Always read through the directions before beginning work. Record results immediately after they have been observed. Do not wait until a later time and then trust to your memory.

Be neat and careful in your work.

Always return reagent bottles to their proper places immediately after using. Keep your desk and apparatus clean and in good order. After being used, apparatus should be thoroughly cleaned before it is put away. Wash it with tap water, using a brush, and finally rinse with distilled water. Greasy materials can be removed with warm water and soap. If these means are not effective, use cleaning fluid (potassium bichromate and concentrated sulphuric acid). Do not throw the cleaning fluid away. Return it to the vessel from which it was taken as it can be used again. Glassware cleaned with cleaning fluid should be rinsed thoroughly with tap water and finally with distilled water.

Do not put a pipette or dropper into any reagent bottle. If a reagent must be measured with a pipette, pour out a small amount into a clean dry beaker, or test tube, and take up with a pipette from this vessel. If more of a given laboratory reagent has been taken from a bottle than is needed, do not return the excess to the original bottle. Be careful not to pour out more of the reagent than you require, but if excess has been taken it should be thrown away.

In using alcohol, ether, acetone, benzol, etc., make sure that no fire is near. Ether vapor is especially dangerous, as it may flow along the desk top for some distance and ignite at a neighboring Bunsen burner. Small. desk fires often may be put out by throwing on sand, or moist sawdust, which should be kept in boxes throughout the laboratory. It is an excellent plan to keep 
a woolen blanket or rug in the laboratory. In case a student's clothing has taken fire he may be wrapped in the blanket and the flames extinguished. Solutions of sodium bicarbonate and of boric acid should also be kept in the laboratory for use if acid or alkali is spattered into the eyes or face.

In testing for substances present in small amounts, make sure that both the liquid to be tested and the precipitating reagent are perfectly clear, filtering them repeatedly if necessary. If uncertain as to the presence of a precipitate, compare with the original solution.

As many of the chemical reactions in physiological chemistry are extremely complex, the student need write equations only when they are specifically called for in the notes. 


\section{CHAPTER I.}

\section{STANDARD ACID AND ALKALI}

1. Calibration of Pipette.-Boil distilled water, allow to cool somewhat and pour carefully into a small vessel which can be closely stoppered. Cool to the temperature of the weighing room. Clean a 25 or 20 c.c. pipette carefully with cleaning fluid and rinse thoroughly. Weigh to the third decimal a carefully cleaned weighing bottle of small size. With the pipette, measure exactly 25 or 20 c.c. of water, which should be at room temperature, into the weighing bottle, stopper quickly and weigh. The difference gives the weight of water delivered by the pipette. Weigh at least three portions. Take the temperature of the water (this should be that of the room). Assume that 1 c.c. of water at room temperature weighs 0.997 grams. Calculate the volume of the pipette. Paste a label on the calibrated pipette and write on it the volume delivered at the specified temperature.

This method of ealibration may be used for pipettes of any size, provided that the total weight does not greatly exceed 100 gms., which is the largest amount which should be weighed on the quantitative balances.

2. Empty your burette exactly to the 50 c.c. mark. From your pipette, run in 25 (or 20) c.c. of water and note the level. Repeat the process, thus calibrating the burette. By emptying the 5 or 10 c.c. pipettes into the burette in a similar manner, calibrate them.

3. Normal Acid and Alkali.-A normal solution of an acid is of such strength that one liter of the acid will contain one gram equivalent of ionizable hydrogen (1.008 g.). A liter of normal hydrochloric acid thus will contain a weight of hydrochloric acid gas equal to its molecular weight, since this amount, 36.46 g., will contain the desired amount of hydrogen. If sul- 
phuric acid is used, the amount required will be $1 / 2$ the molecular weight, since the formula is $\mathrm{H}_{2} \mathrm{SO}_{4}$, and if this weight were taken, it would contain twice as much hydrogen as required. A normal sodium hydrate solution is of such strength that it will correspond exactly to a normal acid solution, - that is, it will exactly neutralize an equal volume of normal acid. Since one molecule of sodium hydrate will neutralize one molecule of hydrochloric acid, a liter of normal sodium hydrate must contain the same number of molecules as a liter of normal hydrochloric. This result will be obtained if the solution contains an amount equal to the molecular weight of sodium hydroxide, or 40 grams, since 40 grams of sodium hydrate will neutralize 36.46 grams of hydrochloric acid. If barium hydrate were used $\mathrm{Ba}$ $(\mathrm{OH})_{2}$, then as was the case with sulphuric acid, $1 / 2$ the molecular weight should be contained in a liter of solution if it is to be exactly normal.

From the amounts required for a normal solution, other strengths such as $\mathrm{N} / 10, \mathrm{~N} / 5$, etc., may be calculated.

The term "normal" also is used in connection with oxidizing and reducing agents. Thus in the estimation of uric acid a $\mathrm{N} / 20$ potassium permanganate solution is used. A "normal" reducing solution must yield one gram equivalent of reducing hydrogen per liter, and a normal oxidizing solution must furnish per liter enough oxygen to oxidize this amount of hydrogen. A normal potassium permanganate solution contains a weight in grams per liter equivalent to $1 / 5$ the molecular weight.

Preparation of Standard Acid and Alkali.-In order to prepare an acid or alkali of known strength it is necessary to start from a substance of known composition and purity. Various methods are used for standardizing, the two most frequently used being the oxalic acid, and the sodium carbonate methods.

4. Oxalic Acid Method.-Oxalic acid combines with varying amounts of water of crystallization. By drying the oxalic acid a day or two in a desiccator over sulphuric acid of specific gravity 1.35 , however, it may be assumed to contain $2 \mathrm{H}_{2} \mathrm{O}$. Sixty-three 
gms. of the erystals so prepared are dissloved in water and made up of 1 liter. This solution may be considered normal and used to standardize an unkuown solution of sodium hydrate, using phenolphthalein as indicator.

5. Sodium Carbonate Method-A more reliable method for preparing a standard consists in heating pure sodium bicarbonate in a platinum dish. The dish is placed in an air bath already heated to $200^{\circ} \mathrm{C}$. and the temperature raised to $270^{\circ}-280^{\circ}$ but not above $300^{\circ}$. It is heated at this temperature for half an hour, then cooled in a desiccator, and before the sodium carbonate is quite cool, transferred to a dry stoppered weighing bottle.

To standardize an unknown acid solution, rapidly weigh 2-3 gms. of carbonate prepared as above, dissolve in $80-100$ c.c. of water, add 2 drops of methyl orange and titrate with the unknown acid.

An exactly normal acid should neutralize pure sodium earbonate in the ratio of 100 c.c. acid to 5.3 gms. carbonate. From the results of the titration, which should be done in duplicate, the strength of the unknown acid may be calculated, and the acid diluted accurately to the required strength. After dilution, the acid should be titrated again against sodium carbonate to make sure that the dilution was accurate.

After making an accurate solution of normal acid $\left(\mathrm{H}_{2} \mathrm{SO}_{4}\right.$ or $\mathrm{HCl}$ ) a solution of normal sodium hydrate may be prepared, using the normal acid as a standard and titrating with alizarine red or methyl red as indicator.

If time does not permit each student to start from oxalic acid or sodium carbonate in the peparation of his normal solutions, standard solutions of $\mathrm{N} / 10$ acid and alkali prepared as above described should be furnished for the purpose of standardizing the solutions made by the class.

Note: In the author's classes it has been customary to furnish to the class $\mathrm{N} / 10$ acid and alkali for the purpose of standardizing? solutions prepared by them as described below, thus saving much time for the class; students then use their own $\mathrm{N} / 10$ solutions in their work. 
Each student should prepare one standard solution. The class may be divided into pairs, one student of each pair preparing a standard acid, his partner a standard alkali. The solutions prepared then ean be used jointly by each pair of students.

6. Preparation of $\mathbf{N} / 10$ Acid.-Read all of section (6) before beginning your work. Conc. hydrochloric acid is about $41 \%$ by volume, that is, 100 c.c. contains 41 grams of hydrochloric acid gas. Calculate the amount of conc. acid required to make 2 liters, 11/2 or 1 liter of N/10 acid, according to the size of the large glass stoppered bottle with which you are provided, and measure this amount of acid into your large bottle. Add distilled water to make the volume up to about 100 c.c. less than the total volume for which you have made your calculation.

With a clean pipette (see note "on cleaning glassware") measure out 25 c.c. of the acid into an Erlenmeyer flask. The pipette either may be dry, or it may be rinsed out once with the solution to be measured.

Add 2-3 drops of alizarine red as indicator, and from a burette (also cleaned as above and either dry or rinsed with $\mathrm{N} / 10 \mathrm{NaOH}$ ) run in $\mathrm{N} / 10$ sodium hydrate. In reading the burette, read the lowest curve of the meniscus. Have your eye on a level with the meniscus. If the acid were exactly $\mathrm{N} / 10$ it would require exactly 25 c.c. of the alkali to neutralize it. Make the titration in duplicate. Duplicates should agree within 0.1 c.c. From the amount of alkali used in titration calculate the amount of dilution necessary to make the acid exactly $\mathrm{N} / 10$.

Example. -25 c.c. acid used. Amt. N/10 alkali to neutralize the acid 27.2 c.c. Thus 25 c.c. of the acid used contains enough hydrochloric acid to neutralize 27.2 of N/10 alkali. To make the acid exactly $\mathrm{N} / 10$ it must be diluted in the ratio of $25: 27.2$.

Measure the total amount of your acid with a large cylinder and calculate the volume of water necessary to dilute it to tenth normal acid. Add this amount of water from a cylinder, and shake thoroughly. Allow to stand for a few minutes, and repeat the titration as above.

If your acid does not check with the $\mathrm{N} / 10$ alkali, correct it 
again as above by the addition of the calculated amount of water, or of the calculated amount of hydrochloric acid in case the solution is now too weak.

Preserve your tenth normal solution for future use.

Rinse out the pipette and burette thoroughly.

7. Preparation of a Standard Alkali.-Read over carefully section (6) on the preparation of a standard acid, as the principles involved are identical with those for the preparation of the alkali.

A concentrated solution of sodium hydrate will be used as a stock solution. The strength of the $\mathrm{NaOH}$ will be found on the bottle. Calculate the amount required to make up 2, $11 / 2$, or 1 liter of N/10 alkali according to the size of the glass stoppered bottle in your desk.

Measure this amount into the large bottle and add enough distilled water to make the volume up to about 100 c.c. less than the total volume for which you have made your calculation.

In titration with alizarine red as an indicator it is necessary to run alkali into acid. Accordingly, measure 25 c.c. of the standard N/10 acid furnished into a clean Erlenmeyer by means of a clean pipette. Add 2-3 drops of alizarine red.

Clean your burette and either dry it or rinse with the solution to be used in it. Where the supply of liquid is adequate, the second method is easier.

Fill the burette with your alkali, and titrate the standard acid. Make a duplicate titration to check your result. The duplicate titrations should agree within 0.1 c.c.

If your alkali were exactly $\mathrm{N} / 10$, it would require 25.0 c.c. to neutralize the 25 c.c. acid. As you have made it up slightly stronger than this, it will require somewhat less than 25 c.c. alkali to neutralize the 25 c.c. of acid.

From the amount of alkali used calculate the amount of dilution necessary to make the alkali exactly $\mathrm{N} / 10$.

Example.-25 c.c. N/10 acid is neutralized by 23.2 c.c. alkali.

Thus 23.2 c.c. of alkali contains enough $\mathrm{NaOH}$ to neutralize 
25 c.c. $\mathrm{N} / 10$ acid. To make the alkali exactly N/10 it should be diluted in the ratio of $23.2: 25$.

Measure the total volume of your alkali in a large cylinder (first returning any of the solution remaining in the burette) and calculate the amount of water necessary to dilute it to tenth normal alkali. Add this amount of water from a cylinder, shake well and allow to stand for a few minutes.

Rinse out the burette with the diluted alkali, and repeat the titration against standard acid.

If your alkali does not check with the standard acid, correct it again by adding the calculated amount of water, or the calculated amount of sodium hydrate if the solution has been made too dilute.

Preserve your tenth normal solution for future use.

\section{Kjeldahl Method for Nitrogen Determination.-}

This method is of utmost importance and is widely used for estimating total nitrogen. The various nitrogen compounds are broken down by heating with concentrated sulphuric acid, the nitrogen being converted into ammonia, and the carbon into carbon dioxide. The ammonia is retained in the solution as $\left(\mathrm{NH}_{4}\right)_{2} \mathrm{SO}_{4}$. It then is liberated by the addition of sodium hydrate and distilled into a known volume of $\mathrm{N} / 10$ hydrochloric or sulphuric acid. The excess of N/10 acid is then determined by titration and the amount of ammonia calculated.

In analyzing liquids it is customary to use 5 or 10 c.c., according to the nitrogen content. If the material is a solid, 1 gram accurately weighed is the usual amount.

With a pipette measure 5 c.c. urine into a 500 c.c. Kjeldahl flask. Add 8-10 g. potassium sulphate which raises the boiling point, 15 c.c. of concentrated $\mathrm{H}_{2} \mathrm{SO}_{4}$ and 2 c.c. of $5 \%$ copper sulphate which acts as a catalyser.

Heat the flask in an inclined position over a small flame until the contents become clear and pale green. There must be no suggestion of yellow and no black specks of unoxidized carbon anywhere in the liquid or on the inner surface of the flask. If 
black specks are present, remove the flask from the flame and by careful shaking, rinse them down. Do not attempt to rinse them down by adding water.

Note: Injurious fumes are given off during the heating in the Kjeldahl analysis, so that it should never be carried out in the open laboratory, but in a well drawing hood, or other arrangement for carrying off the fumes.

When digestion is complete, allow the liquid to cool and about half fill the flask with distilled water. The nitrogen now is in solution in the form of ammonium sulphate. It must be set free as ammonia and driven over by distillation into a known volume of standard acid.

Arrange apparatus (condenser, connections, etc.) to distill off the ammonia. The most satisfactory receiving flask is a Woulff bottle. Connect the delivery end of the condenser with one neck of the Woulff bottle by means of rubber and glass tubing, and a stopper. In the other neck of the Woulff bottle fix a cork through which passes the narrow tube of a $\mathrm{CaCl}_{2}$ tube. Charge the bulb of the $\mathrm{CaCl}_{2}$ tube with glass pearls.

Prepare the Woulff bottle by running into it over the glass pearls in the calcium chloride tube, 50 c.c. $\mathrm{N} / 10$ hydrochloric acid measured with a pipette, add 2-3 drops alizarine red and connect the bottle by the other neck with the delivery tube of the distilling apparatus. Set the digestion flask on a ring over the flame and make sure that all connections fit properly. Add a small quantity of talcum powder to prevent irregular boiling (bumping) but not more than the amount that can be taken up on the tip of a knife blade. Add a drop of alizarine and then about 50 c.c. of sat. $\mathrm{NaOH}$ to neutralize the sulphuric acid. In adding the alkali, the flask should be tilted somewhat and the sodium hydrate poured down the side of the tube so that it will form a layer underneath the acid. This is to avoid possible loss of ammonia. Stopper the flask immediately, make sure that the stopper fits tightly, and rotate the flask gently so as to mix the contents thoroughly. If the liquid does not 
turn pink or purple, add more alkali as above. Distill the liberated ammonia into the standard acid. When about $2 / 3$ of

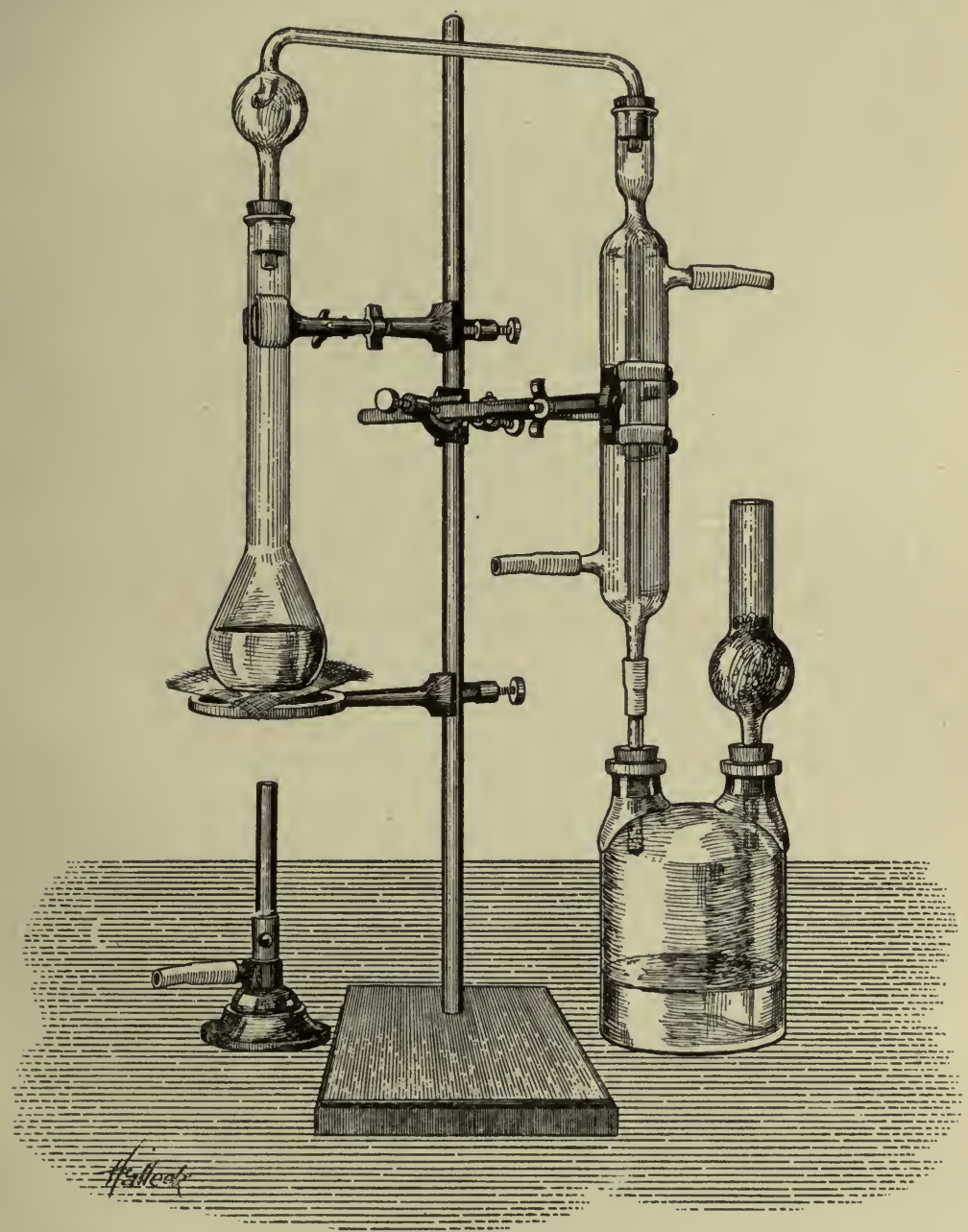

Fig. 3.-Apparatus for Distilling Total Nitrogen by Kjeldahl Method.

the water has distilled over, remove the Woulff bottle and test the next drop of distillate for ammonia by letting it fall into a 
small beaker containing distilled water and a drop of alizarine. If the indicator turns pink, replace the Woulff bottle and continue the distillation until the liquid coming over no longer contains ammonia. When the ammonia is all over, discontinue the distillation, rinse down the glass pearls with distilled water, and titrate the excess of $\mathrm{N} / 10 \mathrm{HCl}$ with standard alkali.

Subtracting the amount of alkali used from the total acid (50 c.c.) gives the volume of acid neutralized by the ammonia.

Multiply this figure by the weight of nitrogen in 1 c.c. of $\mathrm{N} / 10$ ammonia (1.401 mg.). This gives the weight of nitrogen in 5 c.c. urine.

In accurate work, a blank determination should be made to estimate the amount of nitrogen in the reagents used.

9. Indicators.-Very carefully titrate 2-20 c.c. portions of $\frac{\mathrm{N}}{10} \mathrm{HCl}$. with $\frac{\mathrm{N}}{10} \mathrm{NaOH}$, using phenolphthalein as indicator in one titration and methyl orange in the other.

10. Repeat the process using as acid 20 c.c. portions of oxalic acid $0.1 \mathrm{~N}$. Explain.

Why was methyl orange used in standardization of acid by the sodium bicarbonate method above? Why could not phenolphthalein have been used?

By adding properly chosen indicators to solutions of known $\mathrm{pH}$ which are made up to form a series, it is possible to obtain a series of graduated tints, each corresponding to a definite $\mathrm{pH}$. By making up an unknown solution with the same amount of indicator, it is possible to determine its $\mathrm{pH}$ by comparing it with the colors in the standard tubes.

11. Catalysis.-Into each of two Erlenmeyer flasks introduce 5 c.e. of urine and 10 c.c. cone. $\mathrm{H}_{2} \mathrm{SO}_{4}$. To one add two or three crystals of copper sulphate. Boil both in the hood or in a fume flue. Which clears first? Explain.

The catalytic action of enzymes is treated at a later point in the laboratory work. 


\section{Colloids}

12. Solation and Gelation.-Cover a small amount of gelatin in a test tube with water and warm. What happens? Allow to cool. What happens? Repeat the process once or twice. Is the process reversible?

13. Imbibition.-Cut two equal-sized pieces of sheet gelatin about $1 / 4 \mathrm{x}^{1 / 2}$ inch. Put one in a test tube or small beaker of cold water for some time. Compare its size with the piece which has remained dry. Explain.

14. Dialysis.-In a parchment or collodion dialyzing tube place some albumin solution and some sodium chloride solution. Suspend the tube in a beaker of distilled water and let it stand several hours or over night. Test the water in the beaker for chloride (has the chloride dialyzed?), and for albumin by acidifying with a drop or two of dilute acetic acid and boiling; a cloudiness indicates albumin. Has the albumin dialyzed?

15. Suspensoids and Emulsoids.-Prepare two test tubes, one containing 1-2 c.c. of colloidal iron (dialyzed iron) the other 1-2 c.c. of albumin solution. To each add a drop of sodium chloride solution. Which is more easily precipitated by electrolytes?

Emulsoids form gels, suspensoids do not.

16. Reversibility.-Prepare two test tubes, one containing 1-2 c.c. dialyzed iron, the other 1-2 c.c. gelatin solution. Evaporate both to dryness in a water bath or beaker of boiling water. When dry, add water to each (1-2 c.c.). Explain.

17. Diffusion.-Prepare a 4-5 per cent solution of gelatin, by dissolving in warm water. Half fill four or five test tubes with the solution and allow to gel. Pour on the gel 1-2 c.c. of colloidal iron, copper sulphate, congo red, beet juice and any other colored salt solution available. Which are colloids? (Holmes.) 
18. Adsorption.-The student is familiar with a demonstration of adsorption in the decolorization of solutions by means of charcoal.

Place a small amount of dry starch in a test tube and add 2-3 c.c. of a very dilute iodine solution (straw color). Shake well, and allow the starch to settle. Observe the color of the supernatant liquid. What has happened? 


\section{CHAPTER II}

\section{DETECTION OF THE ELEMENTS AND OF INORGANIC SALTS}

The body tissues, foodstuffs, etc., are made up of a large number of elements which are seldom free, but usually combined in the form of organic substances or inorganic salts. The latter are often in more or less firm combination with the organic constituents of the tissues. The inorganic materials often, but not always, can be extracted from tissue material with water or dilute acid. To detect the elements in organic substances it is usually necessary to destroy the organic material by fusing it with an oxidizing agent. Metals or salts making up part of the compound are set free in this manner and can be detected by the usual qualitative tests. The following experiments will demonstrate the presence of the various elements and salts which are found most frequently in the body or in food materials.

I. 1. Carbon.-Place a small quantity of sugar, fat, dry easein or meat which has been thoroughly dried and ground up in a test tube and heat in the Bunsen flame. The material is decomposed, leaving a black residue of carbon.

2. Hydrogen.-Observe the liquid which condenses on the inside of the test tube. This is water. Since the original material was dry, this water must have been formed from the hydrogen in the substance, and oxygen from the substance itself or the air.

3. Oxygen.-There is no simple method for detecting the presence of oxygen in combination. In analysis, the sum of all the other constituents present in a given substance is subtracted from the weight of material used. The difference is taken as the amount of oxygen in the compound. 
4. Nitrogen.-Mix a small portion of casein or meat with soda lime in a test tube and warm gently in the flame. (Soda lime is prepared by mixing solid sodium hydroxide and calcium hydroxide and heating the mixture.) Nitrogen is liberated in the form of ammonia. To detect this compound, moisten a piece of litmus paper with distilled water and hold it in the mouth of the tube. It should turn blue.

Sulphur.-Sulphur may be present as "loosely combined," that is, unoxidized sulphur, or in oxidized form as sulphate.

5. To detect loosely combined sulphur suspend a small amount of casein in water, add sodium hydrate and a few drops of lead acetate and boil. "Loosely combined" sulphur is split off, and combines with the lead to form lead sulphide, which causes the liquid to turn dark brown or black. Add sufficient hydrochloric acid to make the solution acid, and hold in the mouth of the test tube a strip of filter paper moistened with lead acetate solution. The acid liberates hydrogen sulphide gas, which will precipitate lead sulphide as a shiny dark material on the filter paper.

6. Oxidized sulphur is usually present as sulphate. It is detected by precipitation as barium sulphate. Sometimes sulphate can be extracted from a tissue with water or dilute acid. Sometimes it is liberated only when the material is destroyed. (See below under the analysis of special tissues.)

II. Prepare extracts of the following important tissues or fluids and test them for inorganic constituents. Other tissues may be substituted if desired. Directions for the specific tests will be found in Section III, immediately following the methods of preparing the extracts.

Prepare each material for analysis, and so far as is possible, complete the tests upon it before beginning work on another material.

7. Preparation of Muscle Extract.-Heat 100 c.c. of distilled water to boiling and add a small teaspoonful of chopped meat. Dilute about 2 c.c. of $10 \%$ acetic acid with about $18-20$ c.c. of distilled water. The resulting acid will be about $1 \%$. Acidify the water and meat mixture by adding 2-3 drops of the $1 \%$ 
acetic acid prepared, and boil for 2-3 minutes, stirring with a glass rod. If the liquid does not clear, add 3-4 drops more of the acid and boil again. Observe the mixture closely, as the liquid may be quite clear, but appear cloudy from the large number of solid particles suspended in it. Repeat the addition of acid and boiling if necessary. Caution: Addition of too much acid will cause the solution to be muddy in appearance, so that it will not filter clear. This treatment dissolves out a large part of the inorganic salts, and coagulates the protein material. Filter the liquid from the solid residue. Test the filtrate for inorganic materials as described below in Section III.

8. Muscle Residue.-The coagulated protein material and connective tissue also contain inorganic substances, but in organic combination. To detect them it is necessary to destroy the organic compounds by fusion with an oxidizing agent.

Wash the coagulated muscle residue with a little hot water and dry it by pressing between filter papers. Half fill a crucible with fusion mixture. This is a basic oxidizing agent which contains $\mathrm{KNO}_{3}$ and $\mathrm{NaOH}$. The nitrate furnishes nascent oxygen, and the sodium hydrate prevents loss by volatilization of inorganic substances, excepting a portion of the carbonates.

Support the crucible in a clay triangle and melt the fusion mixture (hood) by heating cautiously with the Bunsen flame. Add small portions of the muscle residue until the charred particles do not disappear on heating carefully. When this point has been reached, add a few crystals of fusion mixture, and heat until colorless. Allow the crucible to cool and dissolve the residue in water acidified with nitric acid. The volume should not exceed 100 c.c. Test the solution with litmus (it should be acid) and make phosphate, sulphate and iron tests as described below in Section III. What do you conclude as to the forms in which phosphorus, sulphur and iron are present in muscle tissue?

9. Blood Serum.-Heat 100 c.c. distilled water to boiling, acidify with a few drops of $1 \%$ acetic acid and pour into it slowly 20 c.c. blood serum or blood. The liquid should be faintly 
acid. Test with litmus and add more of the acetic acid if necessary to produce a slightly acid reaction. Boil for a few minutes. If the liquid does not become perfectly clear, add a few drops more of the acid and boil again. Avoid excess of acid, which will cause the liquid to become milky. Filter the clear liquid from the coagulated proteins and test it for inorganic materials as described below in Section III.

10. Bone.-Dissolve a small spoonful of bone ash in 100 c.c. of water acidified with nitric acid. Is a gas given off? If so, what substance is shown to be present in bone? (Remember that in preparing the bone ash much $\mathrm{CO}_{2}$ may have been driven off by heating.) If the bone ash does not all dissolve, add more nitric acid, or filter off the undissolved ash. Test the solution for inorganic materials as described below in Section III. Before testing for calcium, neutralize the solution with ammonia (litmus) and reacidify with $10 \%$ acetic acid, as calcium oxalate is soluble in nitric acid. In case the phosphates thrown down by the ammonia do not all dissolve on the addition of acetic acid, the solution must be filtered before performing the calcium test.

\section{Tests for Inorganic Materials.-}

Use about 3 c.c. of the solutions prepared in Section II for each test. Record results as -, indicating absence; +, indicating traces; and ++ , indicating much. If the liquid only becomes cloudy, report + . If there is a distinct precipitate, report ++ .

11. Chlorides.-Acidify with about $1 / 3$ volume of cone. nitrie acid and add silver nitrate. The presence of much chloride is indicated by a heavy white precipitate. If only traces are present, the solution becomes cloudy and shows a bluish-white opalescence. Write the equation. Confirm the presence of chloride by adding ammonium hydrate until the liquid reacts alkaline to litmus. On adding this reagent, any precipitate of silver chloride will dissolve, but if phosphates are present in quantity, they will be thrown down. If a precipitate forms on the addition of ammonia, filter, and in the filtrate confirm the presence 
of chlorides by acidifying and reprecipitating with nitric acid.

12. Sulphates.-Add dilute hydrochloric acid and barium chloride. Write the equation. The precipitate is barium sulphate.

Note: Sulphates are present only in small amounts in most body tissues and fluids. Unless care is exercised, a slight precipitate of sulphates will be overlooked. The sulphate test should be observed carefully in a good light, and compared with the original solution which is being tested in order to detect a possible slight precipitate.

13. Phosphates.-Add 1-2 c.c. conc. nitric acid-and about 2-3 c.c. ammonium molybdate. Warm carefully until just too hot to be held in the hand, and allow to stand. If phosphates are present, a yellow crystalline precipitate of ammonium phospho-molybdate will gradually settle to the bottom of the test tube. The precipitate may not form for some time, but its appearance often may be hastened by rubbing the inside of the test tube gently with a glass rod. If the precipitate is curdy, add more nitric acid and boil until it dissolves, as this is not the phosphate precipitate.

14. Carbonates.-The carbonates present in muscle and blood were decomposed by boiling with acid, carbon dioxide being given off. Also the carbonates in bone were partly destroyed in the preparation of bone ash. To detect carbonates in bone, add $2-5$ c.c. water to a small amount of ground bone and add a few drops of concentrated nitric acid. Observe the bubbles of $\mathrm{CO}_{2}$. If the mouth of the test tube is held to the ear, the effervescence may be plainly heard.

15. Calcium.-To 10 c.c. of the solution add about 2 c.c. ammonium oxalate and allow to stand for 15 minutes. The precipitate is calcium oxalate. The test must be made in acetic acid solution as nitric acid dissolves calcium oxalate. Write the equation. Use for the magnesium test the portion tested for ealcium.

16. Magnesium.-Filter off any precipitate of calcium oxalate in the liquid tested for calcium. If the filtrate does not come 
through clear, add a pinch of bismuth subnitrate and refilter through the same filter paper. Repeat if necessary until clear. The bismuth subnitrate, which is extremely insoluble, closes up the larger pores of the filter paper, and thus holds back the fine calcium oxalate crystals. To the clear filtrate add a few c.c. of ammonium oxalate and allow to stand for some time, preferably over night, to make sure that all calcium has been removed. After complete removal of the calcium, add ammonium hydrate and ammonium phosphate to the clear filtrate and allow to stand for 15 minutes. The precipitate is magnesium ammonium phosphate. Write the equation. Save the filtrate for the sodium and potassium tests.

17. Iron.-Add hydrochloric acid and potassiun ferrocyanide to the liquid to be tested. In the presence of iron "Prussian blue" is formed. A small amount of Prussian blue in the solution may result only in a green color, due to a combination of blue with the yellow already present in the solution. A green color may thus be taken to indicate a trace of iron. If more than a trace of iron is present a precipitate will form. Write the equation. Compare with a blank test on the reagents used. If iron is found, remember that it may have come from the hemoglobin of the blood present in traces in the tissues.

18. Sodium.-Use one-half the filtrate from the magnesium test. Make sure that all magnesium has been removed by adding a few drops of ammonium phosphate. Divide the filtrate into two portions and reserve one for the potassium test.

To test for sodium, add potassium hydrate a few drops at a time and warm in an evaporating dish until all the ammonia has been driven off. (Test by holding over the dish a piece of red or neutral litmus paper moistened with distilled water.) Pour into a watch glass and concentrate on the steam bath to about $1 / 2$ c.c. There should be no solid material present when the process is stopped. If solid material has appeared in the liquid, the concentration has been carried too far, and the solid should be dissolved up by the cautious addition of water drop by drop, stirring the mixture with a glass rod. Add a few drops of potas- 
sium pyroantimonate solution. If sodium is present a fine white granular precipitate will form in the course of a few minutes and stick to the glass. This test must be made in neutral or alkaline solution. If no precipitate is visible, observe carefully in a good light. Rotate the watch glass earefully so that small particles of precipitate will collect in the center. It often is possible to hasten the formation of a precipitate by rubbing the inside of the containing vessel with a glass rod.

19. Potassium.-From the portion of the liquid reserved in $\mathbf{1 6}$ for the potassium test, remove ammonia as in 18 , but use sodium hydrate instead of potassium hydrate. Add glacial acetic acid until the liquid is acid to litmus. Now add 2-3 drops of sodium cobaltinitrite solution. In the presence of potassium a yellow crystalline precipitate forms at once. 


\section{CHAPTER III}

\section{CARBOHYDRATES}

Study the reactions of the physiologically important carbohydrates in solutions of the pure substances, as this method is more convenient for laboratory purposes than the preparation of the materials from the tissues or body fluids in which they occur.

\section{Monosaccharides}

I. Hexoses.-This group includes the monosaccharides of greatest physiological importance, dextrose, levulose and galactose.

Dextrose--(Glucose.)

1. Solubility.-Test the solubility of dextrose in water, $95 \%$ alcohol and ether. (Be careful of fire.) To test the solubility of a substance in a given solvent, take a small amount of the solid in a test tube and to it add a few cubic centimeters of the liquid. In case the solid does not completely dissolve, filter and test the filtrate for the substance in question to see if any has dissolved. This may be done either by evaporating the solution to dryness, or by chemical means. If there is any doubt as to the solubility of dextrose in the solvents tried, filter and evaporate to dryness on the steam bath. A residue indicates that a portion of the material has dissolved.

Test the solubility of dextrose in alcohol diluted with an equal volume of distilled water. Dextrose is soluble in dilute alcohol. Will it be precipitated from aqueous solution by the addition of alcohol? This is a point of difference from dextrin.

2. Fehling's Test.-Fehling's reagent is made up in two parts, $\mathrm{A}$ and $\mathrm{B}$, which are mixed in equal amounts immediately before using. A contains copper sulphate; B contains sodium hydrate 
and sodium potassium tartrate (Rochelle salt). The solutions are kept separate, as after mixing, a reduction will take place in the course of time, due to the action of the tartrate.

Mix equal portions (about 5 c.c.) of $\mathbf{A}$ and B. A temporary whitish precipitate of cupric hydroxide forms, but dissolves when the liquids are well mixed, the solution becoming deep blue. The cupric hydrate forms a soluble compound with the tartrate present, the tartrate thus serving to hold the cupric hydrate in solution. Heat to boiling. No change occurs, since the cupric hydrate held by the tartrate does not decompose. If the tartrate were not present, the cupric hydrate would be converted into black cupric oxide on boiling. To the hot liquid add a few drops of dilute dextrose solution and boil. If no change is observed, add more dextrose and boil again. Repeat until a reaction is obtained. A precipitate forms which may be yellow at first (cuprous hydroxide). On further boiling this is converted into red or brownish red cuprous oxide. This is one of the most widely used tests for reducing sugars.

For the quantitative method, see chapter on urine.

\section{Benedict's Qualitative Reagent for Sugar.-}

Dissolve $85 \mathrm{~g}$. sodium citrate and $50 \mathrm{~g}$. anhydrous sodic carbonate in 400 c.c. of water. Dissolve 8.5 copper sulphate in 50 c.c. of hot water. Pour the copper sulphate solution slowly, and with stirring, into the alkaline citrate solution. Filter if necessary. Label and preserve.

Heat to boiling about 5 c.c. of Benedict's reagent in a test tube together with a pebble or two, to prevent bumping. Add about 8 drops of sugar solution (or urine) and boil for 2 minutes. If more than two- or three-tenths per cent of sugar is present, the solution will be filled with a colloidal (greenish, yellow or reddish) precipitate. With smaller amounts of sugar the precipitate will usually appear only on cooling (the cooling should not be hastened by immersion in cold water).

Barfoed's T'est is similar in principle to Fehling's. The solution contains copper acetate and acetic acid, the copper being 
reduced by monosaccharides as in the Fehling test. A disaccharide usually gives the Barfoed reaction only on prolonged boiling, which hydrolyzes the disaccharide to simple sugars. If properly applied, Barfoed's test may be used to distinguish between mono- and disaccharides but a concentrated maltose solution will give a quicker reduction than a dilute glucose solution, hence unless experimental conditions, are carefully controlled, erroneous conclusions may result. Place about 5 c.c. of Barfoed's solution in a test tube and heat to boiling. Add diluted dextrose solution (1 to 5) a few drops at a time, heating after each addition. A red precipitate of cuprous oxide forms.

Haines' T'est is similar to Fehling's, except that glycerine is used in place of Rochelle salt and potassium hydroxide in place of sodium hydroxide.

4. The Phenylhydrazine Test depends on the formation of osazones, relatively insoluble compounds of phenylhydrazine and sugars.

In a test tube mix 5 drops phenylhydrazine, 10 drops glacial acetic acid and 15 drops saturated sodium chloride. To the resulting solid add about 3 c.c. of dextrose solution and boil for 2-3 minutes. Allow to stand. Examine crystals under the microscope and draw. The osazones form yellow needles which often group together in rosettes, sheaves, or fans. Many of the sugars may be identified by the crystal form or the melting point of their osazones. If the crystals are slow in forming, place the test tube in a beaker of boiling water for 20 minutes, then allow to cool.

5. Molisch's Test.-To about 5 c.c. of dextrose solution add 2 drops of Molisch's reagent ( $15 \%$ alcoholic $\propto$ naphthol). Incline the test tube and pour concentrated sulphuric acid carefully down the side to form a layer at the bottom of the tube. Notice the reddish violet ring at the junction of the two liquids. This test is extremely delicate, but is given by various substances other than carbohydrates. A negative result is good evidence 
that earbohydrates are absent. A positive test, however, may be due to other substances or to shreds of filter paper (cellulose).

6. Heat a small portion of dry dextrose in a test tube. Note the brown color and the odor of caramel. If a small amount of sugar is heated in a test tube with concentrated potassium hydroxide, a similar result is observed,-the color and odor of caramel appearing. This is known as Moore's test.

7. Optical Activity.-Carefully read the discussion of optical activity in the text. The specific rotation of dextrose is $+52.5^{\circ}$ at $20^{\circ} \mathrm{C}$. provided the concentration is not above $15 \%$. With a polariscope determine the rotary power of the dextrose solution furnished, and calculate the strength of the solution by means of the following formula:

$$
\begin{aligned}
\mathrm{c} & =\frac{\propto \cdot 100}{[\propto]^{20^{\circ} \cdot \mathrm{L}}} \\
\mathrm{D} & =\text { grams per } 100 \text { c.c. } \\
\propto & =\text { observed rotation } \\
{[\propto] \frac{20^{\circ}}{\mathrm{D}} } & =\text { specific rotation }
\end{aligned}
$$

$\mathrm{L}=$ length of observation tube in decimetres

Record the observed rotation. By means of the formula calculate the weight of dextrose per 100 c.c.

If the weight of an unknown sugar in 100 c.c. of solution is known, the specific rotation may be calculated by observing the rotation, and substituting in the following formula:

$$
[\propto]_{\mathrm{D}}^{20^{\circ}}=\frac{\propto}{\mathrm{L} \cdot \mathrm{c}} 100
$$

By referring to a table of specific rotations the sugar under investigation can thus be identified.

8. Fermentation.-The fermentation test is useful in detecting the presence of many sugars. If dry yeast is used, the yeast solution must be "sct" the day before using and kept warm at least over night. Compressed yeast may be used immediately. 
Gently rub up the yeast with the sugar solution to be tested, making a homogeneous mixture.

Fill an Einhorn fermentation tube with this mixture. Make sure that all air is removed from the tube. When the tube is full, pour out most of the liquid from the bulb. Otherwise it will overflow into the incubator in case fermentation takes place. Label the tube with your name and the kind of sugar under examination and place in an incubator at $38^{\circ}-40^{\circ} \mathrm{C}$. until the next class period. Remove the tube and add 3-4 c.c. conc. sodium hydrate from a pipette in such a way that it will enter the upright portion of the tube. Be careful that the gas in the tube does not escape and that no air is admitted. The alkali will absorb the $\mathrm{CO}_{2}$, and the liquid will rise in the tube.

The presence of alcohol in the fermented liquid may be shown by neutralizing and distilling. This is most conveniently done with the combined material of the whole class, the distillation being performed by the laboratory attendant. To about 10 c.c of the distilled liquid add 5-6 drops of $10 \%$ sodium hydrate (no more). Warm to about $50^{\circ} \mathrm{C}$. (This point may be determined by feeling the test tube. Fifty degrees feels hot, but still can be borne by the hand). Add iodine solution drop by drop until the liquid has a faint brown tinge. Allow the tube to stand. Notice odor of iodoform. This is a test for alcohol.

Levulose.-(Fructose.)

Repeat the following tests made on dextrose, using levulose solution.

9. Solubility.-The solubilities of levulose are similar to those of dextrose. The student need not repeat the tests.

10. Benedict's T'est.

11. Phenylhydrazine Test.-Dextrose and levulose form the same osazone, so that they cannot be distinguished one from the other by this test.

12. Molisch Test.

13. Optical Activity.-The specific rotation of levulose is $-93^{\circ}$. Determine the amount of levulose in a solution furnished.

14. F'ermentation.-Levulose ferments readily. 


\section{Galactose.-}

This sugar gives the usual reduction tests and forms an osazone. It may be distinguished from dextrose and levulose by the mucic acid test. Lactose also gives this test, as galactose makes up one-half of the lactose molecule. To distinguish between lactose and galactose one may use the Barfoed test, which reacts with lactose only after prolonged boiling.

15. Mucic Acid Test.-To 50 c.c. of galactose solution add 10 c.c. concentrated nitric acid and evaporate on the water bath to about 3 c.c. or less. The fluid should be clear at this point, and a fine white precipitate of mucic acid should form.

II. Pentoses.-Three pentoses are of physiological interest, arabinose, xylose and ribose. These pentoses give the reduction tests and form osazones. They may be distinguished from dextrose and levulose by two color reactions, the orcin and phloroglucin tests.

Arabinose.-The arabinose may be obtained by hydrolyzing gum arabic by boiling for several hours with $1-2 \%$ sulphuric acid.

16. Orcin Test.-Mix equal volumes (about 2 c.c.) of arabinose solution and concentrated hydrochloric acid, add a few grains of orcin and heat on the water bath. In the presence of a pentose, galactose, or glucuronic acid a red color develops which gradually passes through reddish blue to green. The color alone is not sufficient proof of the presence of a pentose. To confirm the test it is necessary to extract the liquid with amyl alcohol; if pentoses are present, this extract will contain the colored compound which should show an absorption band between the Fraunhofer lines C and D.

17. The phloroglucin test, commonly called Tollen's reaction, is similar to the above test except that phloroglucin is used in place of orcin. The absorption band of the amyl alcohol extract is between $\mathrm{D}$ and $\mathrm{E}$.

18. Final proof of the presence of a particular pentose is obtained by determining the melting point of its osazone. 


\section{Disaccharides}

Saccharose (Sucrose).-Study the properties of saccharose as follows :

19. Solubility.-(See note on solubility determinations under Dextrose). Test the solubility of cane sugar in water, cold alcohol, hot alcohol (do not warm over a flame,-dip the test tube in hot water) and ether.

20. Try Benedict's Test.-Saccharose does not reduce Benedict's solution on short boiling since it has no free aldehyde or ketone group. Try boiling for several minutes. On prolonged boiling some reduction takes place.

21. Phenylhydrazine.-Cane sugar forms no osazone.

22. Inversion of Saccharose.-By boiling with dilute acids saccharose may be broken up into dextrose and levulose. After ascertaining that the saccharose solution will not reduce Benedict's, take another sample of saccharose solution, acidify slightly by adding about 1 c.c. of dilute sulphuric acid and heat on the water bath for 15 minutes. Test again with Benedict's solution. The saccharose will have been hydrolyzed into dextrose and levulose, and the liquid should reduce Benedict's solution.

23. Optical Activity Before and After Inversion.-Determine the rotation of the solution of saccharose. Determine the rotation of a portion of the same solution which has been inverted. The rotation should now be to the left, since the solution contains equal amounts of dextrose and levulose, and the latter is stronger levorotatory than the former is dextrorotatory.

24. Perform a Fermentation Test with Saccharose.

Maltose.-Repeat the following tests performed on saccharose, using a maltose solution:

25. Solubility in water, alcohol and ether.

26. Benedict's.

27. Phenylhydrazine.

28. Fermentation. 
Lactose.-Perform the following tests:

29. Solubility in water, alcohol and ether.

30. Benedict's.

31. Phenylhydrazine.

32. Fermentation.

This test is of importance in identifying lactose. In testing for lactose a control should be run with a lactose solution, to make sure that no lactose splitting enzyme is present in the yeast used.

Lactose may also be distinguished from all other reducing sugar's except galactose by the mucic acid test. Lactose may be distinguished from galactose by the fermentation test.

\section{Polysaccharides}

\section{Starches.-}

33. Potato Starch.-Pare a small potato and with a pocket knife scrape it to a fine pulp. Mix with $300-400$ c.c. of distilled water and whip up thoroughly. Strain through a piece of cheese cloth to remove the coarse particles. The starch granules will settle rapidly to the bottom of the beaker. Wash once or twice by decantation and examine under a microscope. Draw the granules. Filter off a portion and allow it to dry in the air. Make the following tests on starch.

34. Solubility.-Refer to directions for determining solubility as given under Dextrose. In the case of starch, the substance is tested for in the filtrate by chemical means. Test the solubility of starch in cold water, hot water, alcohol, and ether. If uncertain of the result, filter and test the filtrate for starch by the iodine test as described below.

35. Preparation of Starch Solution.-Heat about 75 c.c. of distilled water to boiling. To this add about $1 / 2$ gram of starch which has been rubbed to a thin paste with a small amount of cold water. Boil very slowly for about 15 minutes, replacing water lost by evaporation. Use the resulting opalescent solution for the following tests. 
36. Iodine T'est.-To about half a test tube of distilled water add 3-4 drops of iodine solution. Pour 3-4 drops of this diluted solution into 4-5 c.c. of the starch solution prepared in 35 . Notice the dark blue color. This is due to a starch-iodine compound formed. Iodine gives no color with mono- or disaccharides. To a starch solution colored blue by iodine add 2-3 drops of dilute sodium hydroxide.

To another portion of the blue liquid add alcohol.

37. Perform Benedict's Test.

38. To starch solution add 2-3 c.c. tannic acid solution. The tannic acid solution should be fresh, as tannic acid solutions decompose on standing.

39. Starch will not dialyze through a parchment membrane.

40. Hydrolysis of Starch.-Place about 25 c.c. starch solution in an evaporating dish, add 10 drops of concentrated hydrochloric acid and boil gently. At intervals of about a minute remove a drop or two of the liquid with a glass rod and test with a drop of iodine solution. The starch is broken down into dextrins and finally into simpler carbohydrates (maltoses and glucose). When iodine no longer gives a color, make the hydrolyzed starch solution alkaline with sodium hydrate and perform a Benedict's test. Reduction indicates the maltose or glucose stage.

\section{Dextrines.-}

41. Test the solubility of dextrin in cold water, hot water, alcohol and ether.

42. Iodine Test.-Test a solution of dextrin with a few drops of diluted iodine solution, as under "starch." Refer to the results obtained on the hydrolysis of starch. Try the effect of heat, of alkali and of alcohol on the color produced with iodine.

43. Make a Benedict's Test on dextrin solution.-Remember that if reduction is observed it may be due to the presence of maltose or dextrose as impurities in the dextrin. In the manufacture of dextrin, small amounts of maltose and dextrose are likely to be formed by complete hydrolysis of a portion of the 
starch. Pure dextrin is believed not to reduce Benedict's solution.

44. To dextrin solution add alcohol. Recall the effect of alcohol on dextrose.

\section{Glycogen.-}

This polysaccharide may be obtained from the liver taken from an animal immediately after death, or from fresh oysters. The material must be fresh, as otherwise the glycogen will have been broken down to glucose by the tissue enzymes. The liver (or oysters) is thrown into boiling water slightly acidulated with acetic acid. After boiling a few minutes, the pieces are removed, ground in a mortar with sand, and returned to the water and boiled for several minutes. The glycogen solution is then filtered while hot from the coagulated protein material.

45. Test glycogen solution with iodine. Warm gently by holding the test tube in a beaker of water heated to about $50^{\circ} \mathrm{C}$. Remove as soon as the color disappears and cool under the tap.

46. Test glycogen for reducing sugar with Benedict's solution. Glycogen should not reduce, but a solution prepared as above often will do so, as the result of partial hydrolysis of the material.

47. To 10 c.c. of glycogen solution add about 10 drops of concentrated hydrochloric acid and boil for about 10 minutes. Neutralize carefully with sodium hydrate and repeat the Benedict test. The glycogen is hydrolyzed by the acid. 


\section{CHAPTER IV}

\section{FATS AND PHOSPHATIDES}

\section{Fats}

The fats found in the animal body are mainly the triglycerides of palmitic, stearic and oleic acids. Olive oil and lard are convenient materials for laboratory study. Butter or mutton tallow may be used with equal advantage.

1. Solubility.-Test the solubility of olive oil and lard in water, cold and hot alcohol, ether and chloroform. To 3 c.c. of solvent add 2 drops of oil or a small piece of lard. Note: Do not heat alcohol over the free flame. Heat some water to the boiling point in a small beaker. Turn out the flame and warm the alcohol by dipping the test tube in the hot water.

2. Formation of Crystals.-Many fats will crystallize in small needles from warm alcohol, benzol, etc.

3. Place a drop of oil or a small piece of lard on a filter paper. Observe the transparent spot. This test may be used to detect the presence of a fat in a solvent. On evaporation of the solvent a transparent spot remains on the paper if fat was present in solution.

4. Preparation of a Neutral Oil (Mathews).-Most commercial fats and oils contain some free fatty acid. To get a neutral oil this free acid must be neutralized.

To about 25 c.c. of $95 \%$ alcohol in a small flask add about $1 / 2$ c.c. phenolphthalein solution. Heat to boiling on the steam bath and from a pipette add $0.5 \% \mathrm{NaOH}$ (dilute $10 \%$ twenty times) until a faint pink coloration remains. Now add 6 c.c. of oil. Again heat to boiling, then stopper the flask and shake gently. The alkaline alcohol becomes colorless as the alkali is neutralized by the fatty acids present. Continue the addition of $\mathrm{NaOH}$ as 
above, shaking after each addition until after a vigorous shaking the pink color in the alcohol layer just remains. Allow the two layers to separate in a large test tube. Draw off by means of a pipette the layer of neutral oil, wash it by shaking gently with one or two portions of distilled water in a test tube, and save it for use in the experiments following where neutral oil is called for.

Emulsification.-The property of forming an emulsion is of great importance. The fat is held in suspension in the liquid in the form of minute droplets. Study the conditions affecting emulsification as follows:

5. Shake together thoroughly about 1. c.c. of neutral oil and a few c.c. of water. The oil and water quickly separate into two layers.

6. Repeat 5, first adding a small amount of sodium carbonate solution to the tube. The milky appearance of the water layer indicates that a portion of the oil has emulsified, but the action is not extensive. To neutral oil and water add soap solution and shake. A good emulsion forms, indicating the favorable action of soap on emulsion formation. Upon this property of soap depends in large measure its usefulness in cleansing. Greasy material on the surface washed is emulsified, and the insoluble "dirt" thus liberated and carried away by the water and the "suds."

If rancid oil (oil which contains free fatty acid), water and an alkali, e.g. $\mathrm{Na}_{2} \mathrm{CO}_{3}$ are shaken together a good emulsion is formed, since the mixture contains the soap produced by the action of the fatty acids and the alkali.

Lymph has the power of aiding in the formation of an emulsion. This may be demonstrated (Ranvier's experiment) by bringing drops of oil and lymph together under the microscope. Intense activity will be observed at the juncture of the liquids, and the oil passes into an emulsified state.

7. Examine an emulsion under the microscope. Observe the tiny globules of fat. Examine a drop of milk under the microscope. The fat in milk is emulsified. On standing, a portion of 
it will rise to the surface. This layer containing much fat is known as cream.

8. Saponification of Futs.-If a fat is heated with an alkali it is split into fatty acids and glycerine. The liberated fatty acids unite with any excess of alkali to form soaps, hence the term saponification.

In a flask heat to boiling on the steam bath 50 c.c. of alcoholic sodium hydrate and add 5 c.c. of olive or cottonseed oil and continue heating. The saponification is complete when all the oil has disappearer. This may occur almost immediately. When this point has been reached, transfer to an evaporating dish, add about 75 c.c. of water and heat on the water bath to drive off the alcohol. When the alcohol has been removed (decide this by the odor) divide the liquid into three parts. Filter if necessary. To one portion add dilute sulphuric acid and warm on the water bath. The acid converts the soaps into free fatty acids which form an oily layer at the surface (oleic acid). If a palmitic or stearic acid fat is used, the fatty acids separate in solid form.

To a second portion of the sodium soap solution add an equal volume of saturated sodium chloride. The soap is "salted out" since it is insoluble in sodium chloride solution. In case no precipitate forms evaporate the solution on the water bath to a smaller volume.

To the third portion of the sodium soap solution add calcium chloride solution. The precipitate is calcium soap, which is more insoluble than sodium soap. Hard water, which contains calcium salts, is unsuitable for washing purposes, for the calcium precipitates soap in white flakes, and thus removes it from solution.

9. Acrolein Test.-If a fat is heated with boric acid or potassium bisulphate, acrolein is formed, which may be recognized by its sharp, disagreeable odor. Heat a small amount of lard or olive oil with boric acid or potassium bisulphate in a dry test tube. Continue the heating until the material has become practically dry and note the penetrating odor of acrolein. Repeat the test using a few drops of glycerine in place of the lard. 
Fats give the test because of the glycerine which they contain. Fatty acids and pure soaps do not give the test, a result which might be expected from the fact that they contain no glycerine.

\section{Phosphatides}

The phosphatides contain glycerine, phosphoric acid, choline and fatty acid radicles. Chemically they are closely allied to the fats (see lecture notes). A lecithin will be studied as an example of this group.

Lecithins occur probably in all animal cells and are especially abundant in the brain, which contains also other members of the group of phosphatids and from which these substances may be prepared. For laboratory purposes, lecithin may be obtained from egg yolk.

\section{Lecithin.-}

10. Preparation.-The yolk of one egg is allowed to stand with 30 c.c. ether over night. To 30 c.c. of ether extract of egg yolk add 50 c.c. of alcohol. If a precipitate forms (another extractive substance if present in large amounts may be thrown down by alcohol) filter. Evaporate on the water bath, dissolve the residue in 15 c.c. ether and filter. Add 40 c.c. acetone to the filtrate. The lecithin is precipitated. Filter it off. The filtrate contains cholesterol. Use the lecithin for the following tests :

11. Add a small amount to 1 c.c. of water. Note opalescence. The mixture may be filtered unchanged, although the lecithin does not form a true solution.

12. On a small portion make the acrolein test.

13. Fuse about $1 / 3$ of the lecithin with fusion mixture, dissolve in dilute nitric acid and test for phosphate with ammonium molybdate.

14. Saponify $1 / 3$ of the lecithin by heating in a flask on the water bath with 20 c.c. of alcoholic $\mathrm{NaOH}$ for about 15 minutes, replacing the alcohol if necessary. Evaporate to dryness and dissolve the soap in water. Boil and observe the soap bubbles. 
Acidify with hydrochloric acid and allow to stand, warming if necessary. What is the precipitate?

15. The choline portion of the molecule also may be recognized by appropriate tests.

16. Dissolve $0.5 \mathrm{gm}$. stearic, palmitic or oleic acid in about 20 c.c. warm alcohol. (Be careful of fire.) Add a few drops of phenolphthalein and titrate with $\frac{\mathrm{N}}{10}$ alcoholic $\mathrm{NaOH}$. Calculate the purity of the fatty acid used. 


\section{CHAPTER V}

\section{PROTEINS}

All proteins contain carbon, hydrogen, oxygen and nitrogen; some contain also sulphur, phosphorus, iron or other elements. These elements may be detected by the methods described in the chapter on the elements.

\section{General Protein Reactions}

The general tests for the detection or isolation of the proteins are divided into two groups, color reactions and precipitation reactions.

\section{Color Reactions.-}

1. Biuret Test.-Prepare two test tubes each containing a few cubic centimeters of water. To one test tube add a few drops of egg albumin solution. To each tube add 1-2 c.c. saturated sodium hydrate and a few drops of copper sulphate solution which has been diluted until it has only a faint blue color. Such a copper sulphate solution can be prepared by adding a few drops of copper sulphate solution to a half test tube of distilled water. Notice the lavender or violet color in the albumin tube, and compare it with the color of the control. This reaction is very delicate. It also may be performed by adding the alkali to the protein solution, inclining the test tube slightly and allowing the copper sulphate solution to flow down the side of the tube so as to form a layer on the surface of the liquid. A lavender ring forms at the juncture of the two liquids. The biuret test is given by any substance, protein or otherwise, which contains two $\mathrm{CONH}_{2}$ groups joined either directly or by a single carbon or nitrogen atom, and also by some other similar groupings. The test is named from the fact that it is given by biuret; 
$\mathrm{CONH}_{2} \cdot \mathrm{NH} . \mathrm{CONH}_{2}$, a substance obtained by heating urea to $180^{\circ} \mathrm{C}$.

Repeat the test but first add $\left(\mathrm{NH}_{4}\right)_{2} \mathrm{SO}_{4}$.

2. Millon's Reaction.-To a few cubic centimeters of albumin solution add 2-3 (no more) drops of Millon's reagent (1 pt. by weight of $\mathrm{Hg}$. dissolved in 2 pts. of conc. $\mathrm{HNO}_{3}$ and diluted with two volumes of water). A yellowish or white precipitate forms. Heat the solution carefully. The precipitate will turn pink or red. Repeat the test, first adding sodium chloride solution to the albumin solution. This result should be borne in mind in testing for protein in a liquid containing sodium chloride. Shake up a small amount of dry easein with water and apply the Millon test. Perform the test on a dilute solution of phenol. A beautiful red color results. The reaction is given by substances containing a hydroxyphenyl group $-\mathrm{C}_{6} \mathrm{H}_{4}$. OH. Most proteins contain tyrosine, a substance possessing this grouping, and it is because of the presence of this substance that the proteins give the Millon reaction.

3. Xanthoproteic Reaction.-To a few cubic centimeters of egg albumin solution add concentrated nitric acid. Warm the mixture until the whitish precipitate has dissolved. The solution is yellow. Cautiously add ammonia until the reaction is alkaline and observe the deepening of the color to orange. Repeat the test using a small portion of dry casein. Invert the stoppered bottle of concentrated nitric acid slightly so as to get a drop on the glass stopper. Carefully apply the stopper moistened with nitric acid to a small area on the palm of the hand. In a moment pour on the yellow spot a drop or so of ammonia. Observe the orange spot. Rinse off the hand earefully under the tap. The test is given by substances containing a benzene ring and is due to the formation of certain nitro-compounds. Most proteins contain amino acids in which there is a benzene ring, and thus will respond to this test. This is also true of the proteins of the skin.

4. Hopkins-Cole Reaction.-Mix 2-3 c.c. of albumin solution with an equal volume of glyoxylic acid solution. Add an equal volume of concentrated sulphuric acid, pouring it down the 
inside of the test tube so as to make a layer beneath the aqueous solution. A reddish violet or purple ring will develop at the juncture of the two liquids. Shake the tube gently so as to mix the two layers. The color will spread throughout the entire solution.

This test is often performed by adding glacial acetic acid in place of a solution of glyoxylic acid, as glyoxylic acid is usually present in glacial acetic acid as an impurity. It is more satisfactory, however, to use a solution of glyoxylic acid, which may be prepared by reducing oxalic acid with sodium amalgam or magnesium. The reaction is due to tryptophane, which is present in most proteins.

5. Molisch Test.-The student is already familiar with the Molisch test from his work on carbohydrates. It is given by many proteins and is supposed to indicate the presence of a carbohydrate group in the protein molecule. To about 3-4 c.c. of albumin solution add 2-3 drops of $15 \%$ alcoholic $\propto$ naphthol. Incline the tube and pour down the side a few cubic centimeters of concentrated sulphuric acid to form a layer at the bottom of the tube. Note the result.

\section{Precipitation Tests.}

6. By Heat.-Heat about 3 c.c. of albumin solution to boiling. If no precipitation occurs, add 1 drop of $1 \%$ acetic acid and boil again, repeating the process until coagulation occurs. Repeat the experiment, adding about 1 c.c. saturated sodium chloride solution to the albumin solution. The presence of salts is favorable for coagulation.

In removing the protein from a solution the acid is added after heating, as otherwise acid metaprotein, which does not coagulate on boiling, may be formed. Proteoses, peptones, casein and a few other proteins are not coagulated by heat. Heat another portion of albumin solution, first adding 1-2 drops of concentrated sodium hydrate.

7. Concentrated Mineral Acids.-Prepare three test tubes each containing a few cubic centimeters of albumin solution. Add 
concentrated sulphuric, hydrochloric, and nitric acid respectively drop by drop to the three tubes, and record the results in each. The coagulation with nitric acid may be used to detect extremely small amounts of protein. Dilute 1-2 c.c. of albumin solution with several volumes of water. To a few cubic centimeters of this dilute solution in a test tube, add concentrated nitric acid carefully from a pipette. Caution.-In taking up concentrated acids in a pipette, great care should be exereised to avoid drawing the acid into the mouth. Be sure the point of the pipette is kept well below the surface of the acid, which should have been poured into a clean test tube before being drawn up into the pipette. Do not fill the pipette more than half full of acid.

Run the nitric acid slowly into the albumin solution from the pipette, keeping the point of the pipette at the bottom of the test tube. This facilitates the formation of two layers.

Observe the cloudy ring at the juncture of the two liquids. Performed in this way, the test is known as the Heller Ring test, and is used to detect the presence of protein in urine.

8. Dilute the albumin solution used, and determine the smallest concentration of albumin which will give a positive Heller test.

9. Alcohol.-To a few cubic centimeters of albumin solution add alcohol.

10. Heavy Metals.-To small portions of albumin solution add solutions of copper sulphate, mercuric chloride and lead acetate. Egg white is used as an antidote in cases of poisoning by "blue vitriol," "corrosive sublimate," etc., since it forms insoluble compounds with these metal salts which then can be pumped from the patient's stomach with a stomach pump, or removed by vomiting. The salts of most heavy metals will precipitate proteins, in the same way as those tested above.

11. Acetic Acid and Potassium Ferrocyanide.-To a few cubic centimeters of albumin solution add 5-10 drops of $10 \%$ acetic acid (why?) and then, drop by drop, potassium ferrocyanide. A precipitate forms. Avoid an excess of the reagent, as the precipitate may be redissolved. This test is very delicate. Zine also will give a similar precipitate with ferrocyanide, a fact 
which should be borne in mind if the liquid to be tested has been kept in a zine lined ean.

12. Alkaloidal Reagents.-Prepare six tubes of albumin solution and test the precipitating power of each of the following reagents. Add a few drops of the reagent at a time, until a precipitate forms, avoiding excess, as certain of the precipitates are soluble in excess of the reagent:picric acid, tannic acid and trichloracetic acid. Acidify the remaining three tubes with a few drops of dilute hydrochloric acid before making the tests with: phosphotungstic acid, phosphomolybdic acid and potassium mercuric iodide. An approximate estimation of the albumin in a solution may be made by precipitating the protein in a specially graduated tube, called an Esbach tube, by adding Esbach's reagent, which contains picric and citric acids.

Ammonium or Magnesium Sulphate, Sodium Chloride, etc. "Salting out."

13. To about 3 e.c. of blood serum in a test tube add an equal volume of water, and then powdered magnesium sulphate, (shaking) until no more will dissolve. There should be considerable excess salt in the tube to insure saturation. Globulins are thrown down. Filter off the liquid. Test the precipitate for protein by Millon's reaction. Test a portion of the filtrate by the xanthoproteic test, not forgetting the addition of ammonia. If a precipitate forms, what is it? To the remainder of the filtrate add 1-2 drops of dilute acetic acid. Explain.

14. Repeat 13, using solid ammonium sulphate in the place of magnesium sulphate. A copious precipitate should form. Filter and test filtrate and preeipitate for protein as above. To a portion of the filtrate add 1-2 drops of acetic acid. Explain.

15. To a small portion of blood serum add an equal volume of saturated ammonium sulphate solution, thus producing a mixture half saturated with this salt; this will have the same effect as saturating with magnesium sulphate.

16. Pour 2 or 3 drops of blood serum into a large beaker of distilled water. The cloudiness is due to the precipitation of a protein (globulin) which is soluble in the blood serum because of 
the presence of certain salts. On dilution the protein precipitates. The same results may be obtained if the salts are removed by dialysis.

\section{Individual Groups-Simple Proteins}

Albumins.-The solution of egg white, and also the blood serum used for the general protein tests contained both albumins and globulins. These two classes of proteins may be separated from one another by saturating with magnesium sulphate, or half saturating with ammonium sulphate in neutral solution. Either method causes globulin to precipitate. Saturating with magnesium sulphate will precipitate both groups if the solution is acid. Saturating with ammonium sulphate throws down both groups in neutral solution. Some of the plant albumins and globulins are exceptions to the above statements.

17. Preparation of Albumin Crystals.-Beat up 5 c.c. of white of egg to break the reticulum. Dilute with ten volumes of water and strain through gauze. Add an equal volume of saturated ammonium sulphate. Label with your name and leave in the ice box until the next laboratory period. Filter off the precipitated globulin and to the filtrate add saturated ammonium sulphate until the liquid becomes turbid. Now add distilled water in very small portions until the turbidity has just disappeared. Add drop by drop $10 \%$ acetic acid saturated with ammonium sulphate until a precipitate is obtained. Again place in the ice box until the next period. On standing, the precipitate, which is at first amorphous, will become crystalline. Examine under the microscope. The crystals are small, and look much like sand grains. If similarly prepared, serum albumin and lactalbumin crystals may be obtained.

18. Solubility of Egg Albumin.-Test the solubility of powdered egg albumin in water and $10 \% \mathrm{HCl}$. Recall notes on solubility determinations under dextrose. A filtrate may best be tested for protein by one or more of the color reactions. Recall that albumins are precipitated in neutral solution by saturating with ammonium sulphate, but not with magnesium sulphate, or 
sodium chloride, or on half saturation with ammonium sulphate. If the solution is acid, however, in these latter cases some albumin is precipitated. Recall also that albumin is coagulated by heat and is precipitated by various reagents such as alcohol, mineral acids, etc.

19. Recall the color reactions of the albumins as determined under general protein tests.

20. Coagulation Temperature of Egg Albumin Solution.-Fill a large beaker half full of tap water. In this beaker place a smaller beaker or an Erlemeyer also about half full of water. If the inner vessel is not supported by fitting into the large beaker, arrange the amount of water in this inner vessel so that it will not sink to the bottom of the large beaker. Place a test tube containing about five cubic centimeters of clear, fresh albumin solution in the inner vessel, and add to the albumin solution about 1 c.c. of saturated sodium chloride and a few drops of $1 \%$ acetic acid. While your partner carefully warms the water in the outer beaker, observe the thermometer and the albumin solution. The point at which it becomes cloudy is taken as the coagulation temperature of the protein under examination.

Globulins.-As has been observed above, globulins, at least those of animal origin, are precipitated from neutral solution by saturating with $\mathrm{MgSO}_{4}$ or half saturating with $\left(\mathrm{NH}_{4}\right)_{2} \mathrm{SO}_{4}$.

21. Plant Globulins.-Globulins are found in many plants and may be extracted with dilute salt solutions. An example of this class is Edestin, which may be prepared by extracting hemp seed with $5 \%$ sodium chloride solution.

22. Globulins of the Blood Plasma.-If time permits, the various blood globulins may be fractionally precipitated from blood plasma. Quarter saturation brings down fibrinogen, half saturation precipitates practically all the remaining globulins of which there may be several. The filtrate from the globulins still contains serum albumin, which may be precipitated by acidifying slightly, or by saturating with ammonium sulphate. Fibrinogen also may be precipitated by half saturating with $\mathrm{NaCl}$, differing 
in this respect from the other globulins which require full saturation for precipitation.

Fibrin.-On beating freshly drawn blood with a rod, the fibrinogen separates as shreds of fibrin which gather on the rod, and may be washed free of corpuscles. Examine a piece of fibrin. Note its elasticity. It possesses the general properties of the globulins. Test its solubility in water and $10 \% \mathrm{NaCl}$. Filter off the liquid and test it for protein by an appropriate test to ascertain whether or not any fibrin has dissolved. In choosing your test, recall the effect of sodium chloride on certain of the color tests.

Like other globulins, fibrin is soluble in dilute salt solutions, but complete solution takes place only after several days' standing.

On small pieces of solid fibrin suspended in water try the Millon and the xanthoproteic tests.

Myosin.-This muscle globulin may be prepared from the muscle of a freshly killed rabbit, by grinding in a mortar with $5 \%$ $\mathrm{MgSO}_{4}$, preferably after washing out the blood vessels with physiological saline. The extract contains myosin and also other proteins, e.g. paramyosinogen, from which it may be separated by fractional precipitation with $\mathrm{MgSO}_{4}$. At $50 \%$ concentration, paramysinogen precipitates.

If the concentration of the filtrate is increased to $94 \%$, myosin is thrown down. It may be prepared and its reactions studied if time permits.

The solid meat residue left after extracting the myosin is known as "muscle stroma." It contains some coagulated myosin, and probably members of other groups. Test it by the xanthoproteic reaction and Millon's test.

Neuroglobulins.-Two and perhaps three globulins occur in the brain. They may be prepared by extraction with salt solutions and occur chiefly in the grey matter and the axis cylinders.

Prolamines.-The best known member of this group of alcohol soluble proteins is the gliadin of wheat.

23. Two students may work together. To about 50 gms. of 
flour add in small portions enough water to make a dough. Knead this thoroughly in running water until it is washed free of starch. Press out as much water as possible from the solid residue, which is known as gluten. Add 250 c.c. of $70 \%$ alcohol (prepare this by diluting 185 c.c. desk alcohol, $95 \%$, to 250 c.c.) and knead up thoroughly with the hand. Shake often, and allow to stand until the next laboratory period, or extract for an hour on the water bath. Filter off the gliadin solution from the residue which is mainly glutenin. Evaporate the gliadin solution on the water bath. On the residue make the following tests. Test its solubility in water and $10 \% \mathrm{NaCl}$. Try the Millon and xanthoproteic tests.

24. Glutelins.-The only member of this group which has been carefully studied is the glutenin of wheat. It may be prepared from wheat flour by allowing $0.2 \% \mathrm{KOH}$ to act on the insoluble residue left after the gliadin has been completely extracted. This alkaline extract is then neutralized with $\mathrm{HCl}$, when the glutenin precipitates.

Albuminoids.-These proteins are distinguished by their insolubility in neutral solvents. They form the principal organic components of the supporting framework of the body and of hair, nails, horn, etc.

Keratin.-25. Test the solubility of horn in water and $10 \%$ $\mathrm{NaCl}$.

26. Place a few pieces of horn in a few cubic centimeters each of artificial gastric and pancreatic juice, add a small amount of toluol, and set in the incubator until the next period. Horn is not digested by gastric or pancreatic juice. Conclusions as to the digestibility of horn are drawn from the appearance of the pieces. It is not possible to use the color tests for ascertaining whether or not horn has been digested, as the pancreatic and gastric ferment preparations themselves contain soluble proteins in amounts sufficient to give positive color reactions.

27. Suspend small pieces of horn in water and try the Millon and xanthoproteic tests. Test for loosely combined sulphur. All of these tests should be positive. 
28. Collagen.-Collagen may be prepared from the tendon of Achilles of an ox by dissolving out the mucoid with lime water. After several days' extraction, the residue is mainly collagen. Collagen is insoluble, only slightly digestible and gives certain of the protein color tests. On boiling for some time it is converted into gelatine.

29. Properties of Gelatine.-With gelatine prepared as above or with commercial gelatine perform the following experiments:

Test its solubility in cold water. It only swells up. Heat. What happens? On cooling, if the solution is concentrated, it solidifies to a jelly.

Gelatine digests in gastric and pancreatic juice.

30. Elastin.-Elastin may be prepared from the ligamentum nuchae of the ox. The ligament is cut into small pieces and washed with $10 \% \mathrm{NaCl}$ two or three times, and then with running water for 48 hours. It is then extracted with half saturated lime water for two days. The material then is boiled for at least two hours with dilute acetic acid to remove collagen. The residue is mainly elastin. It is insoluble, somewhat digestible, exhibits a remarkable elasticity, and responds to several of the protein color tests.

Histones.-The globin which forms a part of the hemoglobin molecule is considered to be a histone by most authorities. It is easily split off from hemoglobin by the action of dilute hydrochloric acid.

The histones give the biuret test, usually only a faint Millon reaction, are precipitated from neutral solution by alkaloidal reagents, and form precipitates if added to a protein solution. They are acted on by the digestive juices. They contain a high percentage of diamino acids.

Protamines.-The protamines have been found only in the spermatozoa of fish. The protamines give the biuret test, but most of them give no Millon's reaction. They are precipitated by alkaloidal reagents, and fairly well by neutral salts. They give precipitates with ammoniacal protein solutions. 


\section{Conjugated Proteins}

These compounds are made up of protein combined with some other substance or substances. The non-protein portion is called the prosthetic group:

Glycoproteins.-These proteins are characterized by a high content of a carbohydrate derivative, usually glucosamine. They usually are divided into two groups, mucoids from the tissues and mucins from the fluids and secretions of the body. As these substances are extremely difficult to purify even approximately, there is much disagreement as to their composition and properties.

31. Mucoids or Chondroproteins.-The mucoid obtained by extracting the ligament and tendon in the preparation of albuminoids may be studied if time permits. The mucoid is precipitated by acidifying with acetic acid. It gives the usual protein color tests. By hydrolizing with $10 \% \mathrm{HCl}$ it may be split up. Sulphate and carbohydrate may be detected in the hydrolyzed liquid.

\section{Mucin.}

32. Rinse the mouth carefully with distilled water, and collect a test tube of saliva. If there appear to be solid particles in the liquid it should be filtered. Add $10 \%$ acetic acid as long as a precipitate forms. This precipitate is mucin. It is not soluble in excess of the acid. Allow to settle, decant most of the liquid, and if the precipitate is sufficiently heavy, filter and use small portions of the residue for the following tests. If the precipitate is very slight, use portions of the saliva containing the precipitated mucin from which most of the clear supernatant liquid has been poured off.

33. On a portion of the mucin suspended in water try the biuret and the xanthoproteic tests.

34. Hydrolyze a portion of the mucin by boiling with dilute $\mathrm{HCl}$, and, with portions of the liquid, test for sulphate, and for carbohydrate.

Hemoglobins.-The hemoglobins are compounds consisting 
of a protein combined with hemochromogen or some similar substance. In connection with the study of hemoglobin, some other constituents of blood will be considered. Recall the inorganic materials present in blood as determined in an earlier chapter. What proteins have been observed in the blood earlier in this chapter? In blood are also found fat, sugar, extractives, protein decomposition products and various other substances. The methods for quantitative estimation of these substances will be found in Chap. VI.

Spectroscopic behavior of hemoglobin. Study the spectroscopic behavior of hemoglobin and its derivatives as follows, two students working together.

35. Oxygenation of Hemoglobin.-The color of oxyhemoglobin is a much lighter and more brilliant red than that of hemoglobin. Dilute defibrinated blood with 5 volumes of water. The blood is "laked," that is, the hemoglobin leaves the corpuscles and goes into solution in the water. The liquid, which was opaque, becomes clear. Laking may be brought about in various other ways, as by the addition of a small amount of ether, toluol, etc. Shake up the laked blood with air, closing the tube with the thumb. The color becomes bright red, as the hemoglobin is changed into the brighter colored oxyhemoglobin.

Prepare three test tubes of this diluted blood. To two of them add Stokes' fluid. (This is a mild reducing agent, which contains $2 \% \mathrm{Fe} \mathrm{SO}_{4}, 3 \%$ tartaric acid, and ammonia in amount sufficient to redissolve the precipitate which forms on first adding this reagent. The reagent must be freshly prepared.) The color becomes darker red. The oxyhemoglobin has been reduced to hemoglobin. Pour the blood in one of the Stokes' reagent tubes several times from one test tube to another. The brighter oxyhemoglobin color reappears. Evidently hemoglobin and oxyhemoglobin are readily converted one into the other. Consider this property in connection with the rôle which hemoglobin plays in the organism.

36. Spectroscopic Study of Blood Pigment.-By means of a spectroscope observe the spectrum produced by a luminous gas 
flame and by electric light. The spectrum is continuous. Observe also the incomplete spectrum of the non-luminous bunsen flame. Introduce a small amount of a sodium compound into the flame and observe the yellow band. Such a spectrum is called a bright line spectrum and the particular bands observed are char-

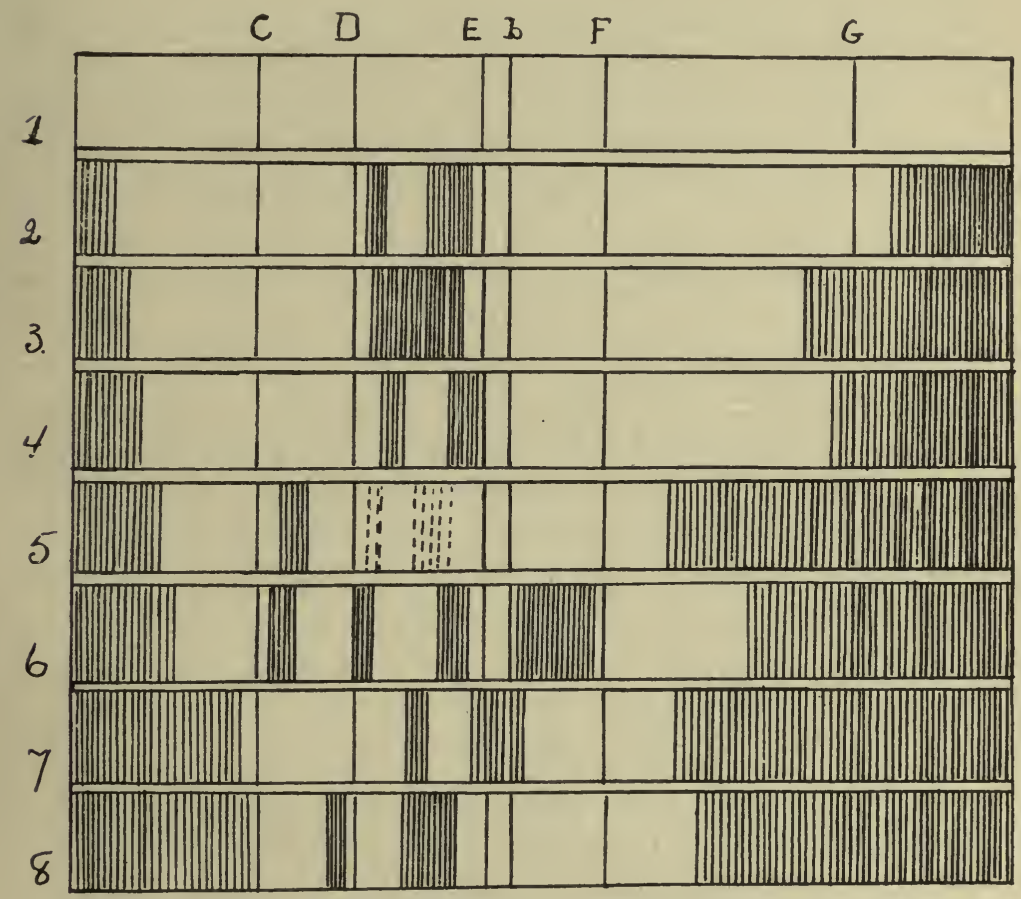

Fig. 2.-Absorption SPECTRA.

1. Spectrum of sunlight showing Frauenhofer lines.

2. Oxyhemoglobin $(0.37 \%)$.

3. Hemoglobin.

4. CO-hemoglobin.

5. Methemoglobin.

6. Acid hematin (in ether).

7. Hemochromogen (reduced alkaline hematin).

8. Acid hematoporphyrin.

acteristic of sodium. Observe the spectrum produced by sunlight. Notice the fine dark lines at various intervals. These are the Frauenhofer lines, and the most prominent of them will be used 
to orient the absorption bands produced by the hemoglobin solutions examined. In making a spectroscopic examination and in charting the result, have the red end of the spectrum always on the left. This avoids confusion and error in interpreting notes. Observe and chart the following lines in your notebook, and indicate them on all your spectrum records. From left to right (beginning in the red) $\mathrm{B}$ and $\mathrm{C}$, two prominent lines in the red; $\mathrm{D}$, a prominent line in the yellow (really two lines if observed with a delicate spectroscope). This line corresponds to the bright lines observed above in the sodium spectrum; $\mathrm{E}$ and $\mathrm{b}$, two prominent lines in the green; $F$ at the beginning of the purple.

37. Examine oxyhemoglobin solutions of various concentrations spectroscopically as directed below. Blood contains between $13 \%$ and $14 \%$ hemoglobin so the percentage concentration of hemoglobin may be calculated for any dilution. The observations are made through a flat sided cell. Each successive dilution may be made by adding an equal volume of distilled water to any volume of the liquid to be diluted. Make observations on blood in the following dilutions: Blood shaken with air and diluted 10 times, 20, 40, 80, 160, 320, 640 and 1280 times. If the solution at a dilution of 1280 still shows two absorption bands, dilute further until only one band remains. Record results.

38. Spectrum of Hemoglobin.-Pour blood diluted 40 times into the cell, reduce it by adding a small amount of Stokes' reagent and observe the spectrum. Record. Repeat with blood diluted 80 and 160 times.

39. Carbon Monoxide Hemoglobin--Prepare a solution of carbon monoxide hemoglobin by diluting blood 160 times, and, in the hood, passing illuminating gas through the liquid. Observe the cherry red color. Observe the solution spectroscopically and chart it, comparing the location of the bands carefully with those given by an oxyhemoglobin solution. To a little of the carbon monoxide hemoglobin solution add Stokes' reagent. Observe that the spectrum does not change, as did that of oxyhemoglobin, which it resembles, under similar conditions. 
Although carbon monoxide forms a fairly stable compound with hemoglobin, still it is possible to remove the carbon monoxide by passing a brisk air stream through the solution for some time. The solution will contain oxyhemoglobin, as can be demonstrated by reducing with Stokes' reagent. The oxyhemoglobin is reduced to hemoglobin.

40. Methemoglobin.-To a small volume of blood diluted ten times add a few drops of a fresh solution of potassium ferricyanide. Methemoglobin is formed. The color becomes a dirty brown. Examine the solution with the spectroscope, diluting somewhat if it is too opaque, and chart. While one student is observing the spectrum, let his partner add Stokes' fluid to the liquid in the cell. Observe that the methemoglobin spectrum gives way to that of oxyhemoglobin, which in turn is replaced by that of hemoglobin. The reducing agent first changes methemoglobin back into oxyhemoglobin (these two compounds contain the same amount of oxygen) from which the oxygen is then removed by the reducing agent, hemoglobin being produced.

41. Acid hematin may be prepared by treating defibrinated blood with half its volume of glacial acetic acid and an equal volume of ether. The liberated hematin dissolves in the ether, and this solution may be used for a spectroscopic examination. It shows a distinct band between $\mathrm{C}$ and $\mathrm{D}$, somewhat nearer $\mathrm{C}$ than the band in the methemoglobin spectrum. A second fainter band appears between $\mathrm{D}$ and $\mathrm{F}$. On dilution this band divides into two, a broad dark band in the green between $\mathrm{b}$ and $\mathrm{F}$, and a narrow fainter band to the left of $\mathrm{E}$. A fourth band may appear on the violet side of $\mathrm{D}$.

42. Acid Hematoporphyrin.-To 5 c.c. of concentrated sulphuric acid in a test tube add 2-3 drops of undiluted defibrinated blood, mixing well after the addition of each drop. Observe the color and chart the spectrum. If the color is too dark, dilute with glacial acetic acid.

Crystallization of Blood Pigment.

43. Hemoglobin Crystals.-Place a drop of defibrinated rat or guinea pig blood on a slide, add an equal volume of water, 
a few erystals of ammonium oxalate, and 1-2 drops ether. Mix and cover. In a few minutes crystals of oxyhemoglobin separate out. Observe under the microscope and draw.

\section{Chemical Tests for Recognizing the Presence of Blood.}

44. Hemin Crystals.-Place a drop or two of blood on a slide. Add one or two crystals of sodium chloride (no more) and rub with a glass rod until the salt has dissolved. Place the slide on a ring about a foot above a small flame and allow the blood to evaporate slowly to complete dryness. Rub the red residue to a powder with a knife blade, collect the powder in a small pile and add a drop of glacial acetic acid from a glass rod. Rub to a paste, place a portion on a clean slide, and add a drop of glacial acetic acid. Cover with a cover slip and cautiously heat over a small flame until the acid begins to boil. Let a drop more of the acid run under the slide and allow to cool. If no crystals form, add another drop or two of acid and repeat the boiling. Examine the crystals of hemin (hematin hydrochloride) under the microscope and draw. The crystals are brownish-red rhomboids.

This test is one of the best methods for detecting small quantities of blood in blood stains. Blood stains on cloth, etc., are soaked in distilled water or alkali, the solution evaporated and treated as above. The formation of hemin crystals is an absolute proof of the presence of blood; it does not, however, distinguish between the blood of man and that of some other animals.

45. Benzidene Reciction.-The benzidene reaction is also one of the most delicate and reliable tests for blood. Different solutions of benzidene vary considerably in sensitiveness, so that in practice a control always should be run, using distilled water in place of blood. Benzidene solutions should be kept in the dark as they are easily altered by the action of light. Mix a saturated solution of benzidene in alcohol or glacial acetic acid, with an equal volume of $3 \%$ hydrogen peroxide and add one cubic centimeter of a dilute blood solution. If the mixture is not already acid, acidify with acetic acid. Note the greenish blue color. This 
test may be performed by adding a small amount of solid benzidene, and glacial acetic acid instead of the benzidene solution.

Guaiac test. This test, if properly performed is extremely delicate. As it is given by substances other than blood, however, conclusions from a positive result should be drawn only with caution. A negative result is conclusive evidence of the absence of blood.

46. Dilute defibrinated blood by adding 2-3 drops to a test tube of distilled water. Add about one-eighth volume of hydrogen peroxide ( 3 vols. per cent) and float on the surface a layer of tincture of guaiacum (or of guaiaconic acid). Note the slow appearance of a green-blue color above the junction of the liquidis. Boil a small volume of blood and repeat the test. It is still positive.

47. On a slice of raw carrot put a little hydrogen peroxide and some of the guaiacum solution. Observe the color. Repeat with boiled carrot. The reaction also is given by milk, pus, saliva, and various other substances, but these substances do not give the test after having been boiled, thus differing from blood. They contain an oxidase which is responsible for the reaction and which is destroyed by boiling. In the case of blood, the reaction depends probably on the catalytic action of the iron constituent of hematin.

48. Catalase. The presence of a catalase in blood may be demonstrated readily. To a little defibrinated blood add hydrogen peroxide. Observe the bubbles of oxygen given off. This reaction is due also in some measure to the blood pigment, but fresh blood contains a catalase in addition. Repeat the experiment with boiled blood. Explain.

49. In testing a blood stain, in addition to the tests given above, a small portion of the fabric should be extracted with glycerol or $0.9 \%$ sodium chloride, and the extract examined with the microscope for corpuscles.

50. An aqueous extract of the stain may be tested for the formation of hemochromogen. If the stain does not dissolve in 
water it may be extracted with acidified alcohol, and the extract examined with the spectroscope for acid hematin.

The identification of a stain as human blood is accomplished by none of the above reactions. The final proof that a blood stain has come from a human subject is obtained by agglutination and hemolysis tests.

51. The globin constituent of hemoglobin may be demonstrated as follows: Corpuscles are washed 2 or 3 times with isotonic salt solution, the corpuscles being separated from the liquid each time by centrifuging. A small quantity of the washed corpuscles is then placed in a test tube, a small amount of alcohol and of hydrochloric acid added and the mixture heated on the water bath. The liquid turns a dark brown color due to the formation of acid hematin, and a precipitate forms which consists of the globin. This may be filtered off, and it will be found to give the usual protein color tests.

Hemoglobin, although erystallizing readily, differs from other crystalloids in not diffusing through an animal membrane.

Phosphoproteins.-The phosphoproteins are compounds consisting of a protein combined with some phosphorus-containing substance other than nucleic acid or lecithin. These proteins often are called nucleoalbumins; they differ from the nucleoproteins by containing no purine bases. One member of the group will be studied, casein from milk. In connection with this substance some other constituents of milk and egg yolk will be considered.

\section{Caseinogen.}

52. Test with litmus the reaction of the milk furnished. Fresh milk is neutral or faintly alkaline.

53. Boil a few cubic centimeters of milk. Observe that the caseinogen does not coagulate. In this respect caseinogen differs from most other proteins. If a skin forms over the surface of the milk, it is due partly to evaporation from the surface of the liquid, partly, perhaps, to coagulation of other milk proteins.

54. To a few cubic centimeters of milk add an equal volume 
of saturated ammonium sulphate. The precipitate consists mainly of caseinogen. In this respect caseinogen resembles the globulins. The member of this group occurring in milk also is precipitated with caseinogen, but its amount is extremely small. Filter the mixture. Divide the filtrate from the caseinogen into two portions. Heat one to boiling, acidifying slightly if necessary. The slight precipitate is lactalbumin. Saturate the second portion with ammonium sulphate. Albumin is precipitated.

55. The usual method for the preparation of caseinogen consists in precipitating with dilute acetic acid. This process is analogous to the clotting of sour milk; by the action of bacteria milk sugar is fermented. The resulting lactic acid, when present in sufficient concentration, causes the caseinogen to precipitate. Dilute 25 c.c. of milk with three times its volume of water, warm slightly (about to body temperature) and add $1 \%$ acetic acid drop by drop, stirring and allowing a short interval to elapse between the addition of successive drops. Caseinogen flocks out as a heavy white precipitate. Add acid until the supernatant liquid is clear. Filter and save both the filtrate $(\mathrm{x})$ for use in 56 and the precipitate. Dissolve the precipitate in $2 \%$ sodium carbonate, and reprecipitate with acetic acid. This reprecipitation is for the purpose of freeing the caseinogen from fat, which is carried down mechanically. It may be repeated several times if a purer product is desired. Redissolve the caseinogen in $2 \%$ sodium carbonate, filter through a wet filter to remove more of the fat, and with this solution perform the biuret, Millon, and xanthoproteic tests, and the test for loosely combined sulphur. The presence of phosphorus in caseinogen already has been observed.

56. The filtrate $(\mathrm{x})$ from the caseinogen prepared in 55 contains lactalbumin and lactoglobulin, whose presence in milk was demonstrated in 54. The filtrate $(x)$ is already acid. Boil it and add $2 \%$ sodium carbonate drop by drop until nearly neutral. If the liquid becomes alkaline, reacidify with a few drops of acetic acid. This treatment removes lactalbumin and lactoglobulin. Filter from the precipitate and test the filtrate with Bene- 
dict's solution for sugar, and for phosphates. Casein is one of the chief constituents of cheese, which also contains much fat.

57. Weigh accurately about $0.2-0.3$ gram dry casein and determine its nitrogen content by the Kjeldahl method.

Recall that unboiled milk gives a positive guaiac test.

Nucleoproteins.-Nucleoproteins are present in all cells. They may be prepared from various organs and tissues.

58. Preparation of nucleoprotein from the pancreas.

Hammarsten's Method.-Grind a piece of fresh beef pancreas in the meat grinder, throw the pulp into 200-300 c.c. of hot water, and boil for about ten minutes. Filter hot. The filtrate will be pale yellow and fairly clear. Cool under the tap, and acidify with sufficient acetic acid to make the concentration of acid $0.5-1.0 \%$. The nucleoprotein is precipitated and quickly settles to the bottom. What other groups of proteins are precipitated in this way? Filter off the precipitate, suspend it in 200 c.c. distilled water, and add ammonia cautiously until the precipitate has just dissolved. Reprecipitate the nucleoprotein with acetic acid as above. If a pure product is desired, the process of dissolving in alkali and reprecipitation with acid should be repeated several times. The precipitate is filtered off, washed with water containing a few drops of acetic acid, and then with about 50 c.c. of hot alcohol in small portions (be careful of fire). Spread the washed nucleoprotein upon a carefully cleaned spot on a tile, and manipulate it to remove the alcohol.

With small portions of the nucleoprotein prepared above perform the Millon, biuret, and xanthoproteic tests.

Note that nucleoprotein was not coagulated by boiling.

59. Fuse a small portion of nucleoprotein with fusion mixture, dissolve the residue in dilute nitric acid and test for phosphorus and iron.

60. Cover a small portion of nucleoprotein with pepsin solution, and incubate at least 24 hours. Note the undigested residue of nuclein.

61. Hydrolysis of nucleoprotein. Mix up the remainder of the nucleoprotein with 10 times its volume of $5 \%$ hydrochloric acid, 
and boil in the hood for about half an hour. To identify the decomposition products of nucleoprotein divide the liquid into four parts and make the following tests: For sugar with Benedict's test; for phosphate; for purine bases. To detect purine bases, add an excess of ammonia, and then a small amount of silver nitrate. Under these conditions purine bases are precipitated as their silver salts.

62. Glycoproteins and many phosphoproteins also are precipitated by acetic acid and dissolve in dilute alkalies. From the above experiments, devise a way to distinguish among glycoproteins, phosphoproteins and nucleoproteins.

Lecithoproteins.-This group of conjugated proteins has not been exhaustively studied. It includes compounds of simple protein with the lecithins, the substances being known as lecithans, and also with some other members of the phosphatid group.

\section{Derived Proteins}

\section{Primary Protein Derivatives.-}

Proteans.-These protein derivatives are formed by the action of very small quantities of acids, of water or of enzymes on most of the proteins. They are characterized chiefly by altered solubilities, as little is known of the proteans.

Metaproteins.-By the further action of weak acids, or of alkalies, products are formed which are readily soluble in weak acids or alkalies, insoluble, however, in neutral solution. The metaproteins are divided into two classes according to the manner of their preparation. These substances often are called albuminates.

\section{Acid Metaprotein.}

Measure 25 c.c. of egg albumin solution (the solution furnished will be egg white diluted 1 to 10 ) into a beaker, add an equal volume of $0.4 \%$ hydrochloric acid and heat on a water bath at $40^{\circ}-50^{\circ}$ for about $1 / 2$ hour. While waiting, the preparation of alkali metaprotein may be started, if time permits the study of both of these substances. At the end of the heating period, boil 
the solution vigorously. This will coagulate any unchanged albumin. The metaprotein is not coagulated by boiling in acid solution. Filter if necessary.

Exactly neutralize the solution of acid albuminate with $0.4 \%$ sodium hydrate. A white precipitate should form. This will redissolve in a slight excess of alkali, so that the process of neutralization must be carried on with great care. Filter off the precipitate and wash it onee with distilled water. Suspend the precipitate in a small amount of distilled water, and with small portions make the following tests:

64. Test for loosely combined sulphur.

65. Add a small amount of $0.4 \%$ hydrochloric acid. The precipitate dissolves. Neutralize carefully with sodium carbonate. The metaprotein is precipitated.

66. Add a small amount of $0.4 \% \mathrm{NaOH}$. The metaprotein dissolves. Neutralize with $0.4 \% \mathrm{HCl}$. It precipitates.

67. Metaprotein may be distinguished from globulins by the fact that it is insoluble in ammonium sulphate of any concentration, whereas globulins dissolve in ammonium sulphate of concentrations less than half saturation, and are precipitated only at this latter concentration.

68. Boil a small portion of the neutral suspension. Cool and add $0.4 \% \mathrm{HCl}$. The metaprotein no longer dissolves in dilute acid,-it has been coagulated by boiling in neutral solution. Recall that it is not coagulated by boiling in acid or alkaline solution.

69. Dissolve the remainder of the acid albuminate in $0.4 \%$ HCl. Apply (i) the biuret and Millon tests. (ii) Try precipitation with mercuric chloride. Acid metaprotein made from meat is known as syntonin.

Acid metaprotein is especially interesting from the fact that it is the first product formed in the digestion of proteins in the stomach by the acid gastric juice.

70. Alkali metaprotein.--Although differing in some important respects, many of the properties of alkali metaprotein are similar to those of acid metaproteins. If time permits, it may be pre- 
pared in the same manner as acid metaproteins, using $0.4 \%$ sodium hydroxide in place of the acid. Its properties are similar to those of acid metaprotein, with the exception that it gives a less definite neutral sulphur test, as a portion of the sulphur is split off in its manufacture.

Coagulated Proteins.-These protein derivatives are produced from proteins by the action of heat, by standing under alcohol, by the action of certain enzymes, and in various other ways. Much of the protein material of our food is coagulated by cooking before ingestion.

71. Measure about 10 c.c. of undiluted egg white into a thinwalled test tube and immerse the tube in boiling water for $6-8$ minutes. Observe the progress of coagulation. Remove the coagulated protein from the tube by means of a glass rod, or the wire handle of a test tube brush.

With small pieces, test its solubility in water and $10 \% \mathrm{NaCl}$.

72. In each of two test tubes put small pieces of the egg white. To one add $0.5 \% \mathrm{NaOH}$, to the other $0.2 \% \mathrm{HCl}$. Warm to $40^{\circ}$ for some time. What happens? Neutralize each carefully. The precipitate indicates that the protein was converted into metaprotein.

73. Place pieces of egg white in each of two test tubes and test their digestibility by pepsin and trypsin, adding a drop of chloroform and a little toluol and digesting several hours in the incubator.

74. With a small piece of egg white in water try Millon's reaction and the xanthoproteic test.

75. Biuret test. Partly dissolve a piece of egg white in coneentrated $\mathrm{NaOH}$. Cautiously add very dilute copper sulphate. Observe the lavender biuret color. From the above results it appears that coagulated albumin still belongs to the protein group. The exact nature of the alterations in the protein molecule brought about by coagulation is not understood.

\section{Secondary Protein Derivatives.}

Proteoses.-The intermediary products of protein hydrolysis are somewhat arbitrarily subdivided into various groups 
distinguished by the concentration of ammonium sulphate necessary to cause their precipitation and by other similar characteristics. Products which are not precipitated by ammonium sulphate are said to have passed beyond the proteose state. "Witte's peptone" and "Armour's peptone" are in reality mixtures of proteoses and peptones. The former is mainly proteose, the latter mainly peptone.

76. Dissolve about a half teaspoonful of "Witte's peptone" in sufficient water to cause solution. Boil to coagulate any unchanged protein. Filter if necessary. Saturate the hot solution with ammonium sulphate. Proteoses are precipitatcd. Remove the precipitate either by filtering, or, if it has formed in clumps, by collecting with a glass rod or with a small watch glass. If the latter method has been employed, filter the liquid to remove any remaining proteose, and reserve the filtrate for the study of peptones below. Press the proteose precipitate between filter papers to remove as much sulphate as possible, and dissolve in a small amount of water. Add solid barium carbonate in excess and boil. Filter from the precipitated barium sulphate and use the filtrate which contains a mixture of proteoses, for the following tests :

77. Biuret, Millon, Adamkiewicz.

78. Precipitation with concentrated $\mathrm{HNO}_{3}$. If a precipitate forms, it consists of the so-called primary proteoses, the only members of this group which are precipitated by nitric acid. Performed as a "ring test," this test is known as Heller's ring test.

79. Precipitate with picric acid. Warm. What happens? Cool.

80. Note that proteoses are not coagulated by boiling, even in acid solution.

81. The lower members of the proteose group are somewhat diffusible through an animal membrane.

Peptones.-The filtrate from proteoses reserved above which contains peptones, or preferably a solution of Armour's peptone may be examined for peptones. After removal of proteoses by 
saturating with ammonium sulphate, concentrate to a small volume, cool, pour off the liquid from the ammonium sulphate crystals, and boil it with solid barium carbonate until sulphate is completely removed. Filter and test the filtrate for the properties of peptones.

82. Perform the biuret, Millon and Adamkiewicz tests. The biuret gives a redder color than with proteins. The Millon and Adamkiewicz tests are usually faint or negative, since tyrosin and tryptophane are split off early in the disintegration of protein.

83. Observe that peptones are not precipitated by concentrated nitric acid.

84. Try precipitation with picric acid, and with potassium ferrocyanide in a solution made acid with acetic acid.

85. Recall that peptones are not coagulated by boiling.

86. The peptones diffuse through an animal membrane more readily than do the proteoses. Since proteins do not diffuse, a mixture of protein and peptones may be freed from peptones by dialysis.

Peptids.-Those decomposition products of the proteins which are made up of a relatively small number of amino acids are known as peptids,-e.g. di-, tri-, tetra-, poly-peptids, etc., according to the number of amino acids making up the peptid molecule. The study of this group of protein derivatives requires an amount of time obtainable only in a more specialized course.

Amino Acids.-The detailed study of these final products of protein hydrolysis, as with the peptids, requires more time than can be devoted to the subject in a general course in biochemistry. Certain members of the group will be studied, however.

87. The student should be furnished with a mixture of protein decomposition products obtained by digesting protein material for several days with trypsin. Test the liquid with the biuret reaction. The result will give an idea of the stage to which the digestion has progressed. Nearly neutralize 100 c.c. of the diges- 
tion mixture with dilute hydrochloric acid. When nearly neutral, exactly neutralize with $0.2 \% \mathrm{HCl}$ or with $0.2 \%$ sodium carbonate to precipitate metaprotein. Filter, if necessary and concentrate the filtrate, being careful to make the solution slightly acid with acetic acid, as heating in alkaline solution will cause decomposition of the amino acids. The concentration may be carried on over a free flame or wire gauze until the liquid becomes fairly concentrated. The process then should be continued on a water bath. To the resulting syrupy liquid, add alcohol as long as a precipitate of proteoses and peptones forms. Remove as much as possible of this sticky precipitate, warm slightly, and filter. The alcoholic filtrate contains leucine and tyrosine. Concentrate the alcoholic solution on the water bath and allow it to stand in a cool place (over night if possible). Tyrosine, being the more insoluble of the two amino acids, crystallizes first. Leucine comes down more slowly. When a good crop of crystals has been obtained, filter them off, add a small amount of water and warm gently. Leucine goes into solution while tyrosine remains undissolved. Concentrate the leucin-filtrate and allow it to stand (over night if necessary).

(1) Tyrosine.

88. Observe the crystal form with the microscope. Tyrosine crystallizes in fine needles, which often group into rosettes or sheaves. If the crystals are not well formed, add a drop of water, warm, and allow to cool slowly.

89. Observe that tyrosine is quite insoluble in cold water, but much more soluble in hot.

90. Perform Millon's test with a small amount of tyrosine.

(2) Leucine.

91. Observe the crystals under the microscope (broad plates).

92. Record solubility of leucine in cold water and hot water.

93. Salts of Amino Acids.-The copper salts of the amino acids are extremely serviceable in separating these compounds, as their solubilities vary considerably. To a solution of either tyrosine or leucine, preferably the former, in hot water, add a few drops of diluted copper sulphate solution. Observe the blue 
color of the copper salt of the amino acid. These salts may be prepared by boiling the amino acid with a neutral suspension of freshly precipitated copper oxide.

94. Preparation of Cystin from Wool.-The student will be furnished with a mixture resulting from the hydrolysis of wool with concentrated hydrochloric acid. To about 50 c.c. of the liquid add solid sodium acetate until congo red paper no longer indicates an acid reaction. This indicator is blue in acid solution, red in alkaline. A precipitate consisting mainly of cystin is thrown down. If the precipitate is not heavy, allow the mixture to stand. Filter off the precipitate, wash with a little cold water, and dissolve in a small volume of $5 \%$ hydrochloric acid. If the solution is dark colored, boil with animal charcoal until a small filtered portion is colorless or pale yellow. Filter, and to the hot solution add hot sodium acetate solution. The cystin will separate out in crystal form as large pentagons, hexagons or plates.

95. Examine the eystin erystals under the microscope.

96. Dissolve a small amount of cystin in caustic soda, add lead acetate and boil. Observe the positive "neutral sulphur" test and recall that as given by proteins, this test depends upon their content of cystin or cystein. 


\section{CHAPTER VI}

\section{MICROCHEMICAL METHODS FOR BLOOD ANALYSIS}

The microchemical methods for the estimation of sugar, urea, creatinine, etc., in the blood have become important as aids in diagnosis. Folin and hi: co-workers have been conspicuous in developing satisfactory methods of analysis. The most important of these are given here.

For the final estimation of nitrogen, the well known Nessler method is used, modified to meet the conditions of these specific methods.

The readings are made in a Duboseq colorimeter. If the students are not familiar with the use of this instrument it should be demonstrated by the instructor.

Folin's Modified Nessler's Reagent (Laboratory Manual, 1921)._- "This reagent is essentially a solution of the double iodid of mercury and potassium $\left(\mathrm{HgI}_{2}, 2 \mathrm{KI}\right)$ containing sodic or potassic hydrate. A stock solution of the double iodid is best prepared as follows:

"Transfer $150 \mathrm{~g}$. of potassium iodid and $110 \mathrm{~g}$. of iodin to a 500 c.c. Florence flask; add 100 c.c. of water and an excess of metallic mercury $140 \mathrm{~g}$. to $150 \mathrm{~g}$. Shake the flask continuously and vigorously for 7 to 15 minutes or until the dissolved iodin has nearly all disappeared. The solution becomes quite hot. When the red iodin solution has begun to become visibly pale, though still red, cool in running water and continue the shaking until the reddish color of the iodin has been replaced by the greenish color of the double iodid. This whole operation usually does not take more than 15 minutes. Now separate the solution from the surplus mercury by decantation and washing with liberal quantities of distilled water. Dilute the solution and washings to a volume of two liters. If the cooling was begun 
in time the resulting reagent is clear enough for immediate dilution with $10 \%$ alkali and water, and the finished solution can at once be used for Nesslerizations.

"The cost of the chemicals called for in this rather interesting process of making Nessler's solution is less than when starting with mercuric iodid and the disagreeable impurities present in many samples of mercuric iodid are avoided. From the stock solution of mercuric potassium iodid, made as described above, prepare the final Nessler solution as follows:

"From completely saturated caustic soda solution containing about $55 \mathrm{~g}$. of $\mathrm{NaOH}$ per 100 c.c. decant the clear supernatant liquid and dilute to a concentration of $10 \%$. (It is worth while to determine by titration that a $10 \%$ solution has been obtained with an error of not over 5\%.) Introduce into a large bottle 3,500 c.c. of $10 \%$ sodic hydrate solution, add 750 c.c. of the double iodid solution, and 750 c.c. of distilled water, giving 5 liters of Nessler's solution.

"In the absence of modifying circumstances, such as the presence of much acid or alkali, this reagent should be added in the proportion of 10 c.c. per 100 c.c. of the volume to which the Nesslerized solution is to be diluted. As a general rule the volumetric flask (or volumetric test tube) should be at least two-thirds full before adding the Nessler reagent. If attention is not given to this detail turbid mixtures are obtained, and turbid solutions must never be used for color comparisons."

Preparation of Protein-Free Blood Filtrates. (Folin and Wu: Jour. Biol. Chem., xxxviii, 81, 1919).- The blood filtrate, the preparation of which is described below, is suitable for the determination of nonprotein nitrogen, urea, uric acid, creatinin, creatin and sugar.

The blood should be collected over finely powdered potassium oxalate, about $20 \mathrm{mg}$. for 10 c.c. of blood. It is important not to use unnecessarily large amounts of oxalate because the excess makes the complete coagulation of the proteins more difficult and also interferes more or less with the uric acid precipitation. 
Reagents required for the precipitation of the proteins:

1. A $10 \%$ solution of sodium tungstate. Some sodium tungstates, though labeled c. p. are not serviceable for this work. They usually contain too much sodium earbonate. The c.p. sodium tungstate made by the Primos Chemical Company, Primos, Pa., is satisfactory.

2. A two-thirds normal sulphuric solution, $35 \mathrm{~g}$. of concentrated c.p. sulphuric acid diluted to a volume of 1 liter, will usually be found to be correct; but it is advisable, indeed necessary, to check it up by titration. The two-thirds normal acid is intended to be equivalent to the sodium content of the tungstate so that when equal volumes are mixed substantially the whole of the tungstic acid is set free without the presence of an excess of sulphuric acid. The tungstic acid set free is nearly quantitatively taken up by the proteins and the blood filtrates obtained are therefore only slightly acid to congo red paper.

Transfer a measured quantity (5 to 15 c.c.) of oxalated blood to a flask having a capacity of fifteen to twenty times that of the volume taken. Lake the blood with seven volumes of water. Add one volume of $10 \%$ solution of sodium tungstate $\left(\mathrm{Na}_{2} \mathrm{WO}_{4}, 2 \mathrm{H}_{2} \mathrm{C}\right)$ and mix. Add from a graduated pipette or burette, slowly and with shaking, one volume of two-thirds normal sulphuric acid. Close the mouth of the flask with a rubber stopper and shake. If the conditions are right, hardly a single air bubble will form as a result of the shaking. Let stand for 5 minutes; the color of the coagulum gradually changes from bright red to dark brown. If this change in color does not occur, the coagulation is incomplete, usually because too much oxalate is present. In such an emergeney the sample may be saved by adding $10 \%$ sulphuric acid, one drop at a time, shaking vigorously after each drop, and continuing until there is practically no foaming and until the dark brown color has set in.

Pour the mixture on a filter large enough to hold it all. This filtration should be begun by adding only a few e.c. of the mix- 
ture down the double portion of the filter paper and withholding the remainder until the whole filter has been wet. Then the whole of the mixture is poured on the funnel and covered with a watch glass. If the filtration is made as described the very first portion of the filtrate should be clear as water and no refiltering is necessary.

It will be noted that the precipitation is not made in volumetric flasks. By the process described 6 or 7 or 11 or 12 c.c. of blood can be used, whereas with volumetric flasks one is compelled to use 5,10 or 20 c.c. because flasks suitable for other volumes are not available. Special graduated "blood pipettes," made by Emil Greiner Co., New York, are very useful for the measurement of the blood, the tungstate and the acid.

The protein blood filtrates are not acid enough to prevent bacterial decomposition. If the filtrates are to be kept for any length of time, more than two days, some preservative, a few drops of toluene or xylene should be added.

Nonprotein Nitrogen Determination. (Folin and $\mathrm{Wu}$ : Jour. Biol. Chem., xxxviii, 90, 1919).-The acid digestion mixture is made as follows: Mix 300 c.c. of phosphoric acid syrup (about $85 \% \mathrm{H}_{3} \mathrm{PO}_{4}$ ) with 100 c.c. of concentrated sulfuric acid. Transfer to a tall cylinder, cover well to exclude the absorption of ammonia, and set aside for sedimentation of calcium sulfate. This sedimentation is very slow, but in the course of a week or so the top part is clear and 50 to 100 c.c. can be removed by means of a pipette. (It is not absolutely necessary that the calcium should be thus removed, but it is probably a little safer to have it done.) To 100 c.c. of the clear acid add 10 c.c. of $6 \%$ copper sulfate solution and 100 c.c. of water. Two c.c. of this solution are substantially equivalent to 1 c.c. of the acid solution previously described by Folin and Denis.

Introduce 5 c.c. of the protein-free blood filtrate into a dry 75 c.c. test tube graduated at 35 c.c. and at 50 c.c. Add 1 c.e. of the sulfuric-phosphoric acid mixture described above. Add a dry quartz pebble and boil vigorously over a microburner until 
the characteristic dense acid fumes begin to fill the test tube. T'his is usually accomplished in from 3 to 7 minutes. When the fumes are unmistakable, cut down the size of the flame so that the contents of the tube are just visibly boiling, and close the mouth of the test tube with a watch glass or a very small Erlenmeyer flask. Continue the heating very gently for 2 minutes from the time the fumes began to be unmistakable, even if the solution has become clear and colorless at the end of 20 to 40 seconds. If the oxidations are not visibly finished at the end of 2 minutes the heating must be continued until the solution is nearly colorless. Such cases are very rare; the oxidation is almost invariably finished within the first minute. Allow the contents to cool for 70 to 90 seconds and then add 15 to 25 c.c. of water. Cool further, approximately to room temperature, and add water to the 35 c.c. mark. Add, preferably with a pipette, 15 c.c. of Nessler solution. Insert a clean rubber stopper and mix. If the solution is turbid, centrifuge a portion before making the color comparison with the standard. The standard most commonly required is $0.3 \mathrm{mg}$. of $\mathrm{N}$ (in the form of ammonium sulfate) in a 100 c.c. flask. Add to it 2 c.c. of the sulfuric-phosphoric acid mixture, about 50 c.c. of water, and 30 c.c. of Nessler solution. Fill to the mark and mix. The unknown and the standard should be Nesslerized at approximately the same time. If the standard is set at $20 \mathrm{~mm}$. for the color comparison, 20 divided by the reading and multiplied by 30 gives the nonprotein nitrogen in $\mathrm{mg}$. per 100 c.c. of blood.

Determination of Urea by Urease Decomposition and Distillation. (Folin and Wu. Jour. Biol. Chem., xxxviii, 94, 1919). -Transfer 5 c.e. of the tungstic acid blood filtrate to a clean and dry Pyrex ignition tube (capacity about 75 c.c.). The graduated Pyrex tubes recommended for the nonprotein nitrogen determination should never be used for urea determinations, because they have contained Nessler solutions and Nessler solutions leave behind films of mercury compounds which destroy the urease. If those tubes must be used, they should first be washed with nitric acid to remove the mercury films. Add 
to the blood filtrate two drops of the pyrophosphate solution described in the method for urea in urine (Folin), or two drops of a molecular 0-phosphate solution ( $1 / 3$ molecular monosodium phosphate plus $2 / 3$ molecular disodium phosphate). Then add 0.5 to 1 c.c. of the urease solution described in the method for urea in urine (Folin) and immerse the test tube in a beaker of warm water and leave it there for 5 minutes. The temperature of the water is not very important but should not exceed $55^{\circ} \mathrm{C}$. The warm water can perhaps scarcely be said to be essential, for the hydrolysis is very rapid at room temperature, but we nevertheless much prefer to use it. If no hot water is used, continue the digestion for 10 to 15 minutes, or as much longer as is convenient. The ammonia formed can be conveniently and quickly aerated into a second test tube. Add a little paraffin oil. 1 or 2 c.c. of $10 \%$ sodium hydroxide are added and the ammonia is aspirated int: a test tube graduated at 25 c.c. and containing 2 c.c. of 0.05 normal hydrochloric acid. Consult the instructor as to the connections for aeration. The two test tubes are connected on the same principle as the cylinder and bottle in the figure given in the chapter on urine analysis (p. 323) in the aeration method for ammonia (Folin). Run the air current slowly for one minute and then rapidly for 10-15 minutes. Rinse the delivery tube, dilute to about 20 c.c. and add 2.5 c.c. of the Nessler solution. Fill to the 25 c.c. mark and compare in the colorimeter with a standard containing $0.3 \mathrm{mg}$. of $\mathrm{N}$ in a 100 c.c. flask and Nesslerized with 10 c.c. of the Nessler solution. The standard and unknown should always be Nesslerized as nearly simultaneously as practicable.

Calculation.-Multiply 20 (the height of the standard in $\mathrm{mm}$. ) by 15 and divide by the colorimetric reading to get the urea nitrogen per 100 c.c. of blood. The reasons for this calculation are, of course, to be found in the fact that the standard containing $0.3 \mathrm{mg}$. of $\mathrm{N}$ is diluted to 100 c.c. while the unknown, which corresponds to 0.5 c.c. of blood, is diluted to only 25 c.c. The only precaution which experienced investigators are likely to 
overlook is that the rubber tubing used for connections needs to be rinsed with water before being used the first time, and, later also, if the tubing has been idle for any length of time. The talcum powder with which the inner and outer surface of rubber tubing is coated is probably the source of the trouble in the case of new rubber tubing. It is probably contaminated with ammonia.

Determination of Preformed Creatinine.-Transfer 25 (or $50)$ c.c. of a saturated solution of purified picric acid to a small, clean flask, add 5 (or 10 ) c.c. of $10 \%$ sodium hydroxide, and mix. Transfer 10 c.c. of blood filtrate to a small flask or to a test tube, transfer 5 c.c. of the standard creatinine solution described below to another flask, and dilute the standard to 20 c.c. Then add 5 c.c. of the freshly prepared alkaline picrate solution to the blood filtrate, and 10 e.c. to the diluted creatinine solution. Let stand for 8 to 10 minutes and make the color comparison in the usual manner, never omitting first to ascertain that the two fields of the colorimeter are equal when both cups contain the standard creatinine picrate solution. The color comparison should be completed within 15 minutes from the time the alkaline picrate was added; it is therefore never advisable to work with more than three to five blood filtrates at a time.

When the amount of blcod filtrate available for the creatinine. determination is too small to permit repetition it is of course advantageous or necessary to start with more than one standard. If a high creatinine should be encountered unexpectedly without several standards ready, the determination can be saved by diluting the unknown with an appropriate amount of the alkaline picrate solution-using for such dilution a picrate solution first diluted with two volumes of water-so as to preserve equality between the standard and the unknown in relation to the concentration of picric acid and sodium hydroxide.

One standard creatinine solution, suitable both for creatinine and for creatine determinations in blood, can be made as fol- 
lows: Transfer to a liter flask 6 c.c. of the standard creatinine solution used for urine analysis (which contains $6 \mathrm{mg}$. of creatinine); add 10 c.c. of normal hydrochloric acid, dilute to the mark with water, and mix. Transfer to a bottle and add four or five drops of toluene or xylene. 5 c.c. of this solution contain $0.03 \mathrm{mg}$. of creatinine, and this amount plus 15 c.c. of water represents the standard needed for the vast majority of human bloods, for it covers the range of 1 to $2 \mathrm{mg}$. per 100 c.c. In the case of unusual bloods representing retention of creatinine, take 10 c.c. of the standard plus 10 c.c. of water, which covers the range of 2 to $4 \mathrm{mg}$. of creatinine per 100 c.c. of blood; or 15 c.c. of the standard plus 5 c.c. of water by which 4 to $6 \mathrm{mg}$. can be estimated. By taking the full 20 c.c. volume from the standard solution at least $8 \mathrm{mg}$. can be estimated; but when working with such blood it is well to consider whether it may not be more advantageous to substitute 5 c.c. of blood filtrate plus 5 c.c. of water for the usual 10 c.c. of blood filtrate.

Calculation.-The reading of the standard in $\mathrm{mm}$. (usually 20) multiplied by $1.5,3,4.5$, or 6 (according to how much of the standard solution was taken), and divided by the reading of the unknown, in mm., gives the amount of creatinine, in mg. per 100 c.c. of blood. In connection with this calculation it is to be noted that the standard is made up to twice the volume of the unknown, so that each 5 c.c. of the standard creatinine solution, while containing $0.03 \mathrm{mg}$., corresponds to $0.015 \mathrm{mg}$. in the blood filtrate.

Determination of Creatine Plus Creatinine-Transfer 5 c.c. of blood filtrate to a test tube graduated at 25 c.c. These test. tubes are also used for urea and for sugar determinations. Add 1 c.c. of normal hydrochloric acid. Cover the mouth of the test tube with tin foil and heat in the autoclave to $130^{\circ} \mathrm{C}$. for 20 minutes or, as for the urea hydrolysis, to $155^{\circ}$ C. for 10 minutes. Cool. Add 5 c.c. of the alkaline picrate solution and let stand for 8 to 10 minutes, then dilute to 25 c.e. The standard solution required is 10 c.c. of creatinine solution in a 50 c.c. 
volumetric flask. Add 2 c.c. of normal acid and 10 c.c. of the alkaline picrate solution and after 10 minutes standing dilute to 50 c.c. The preparation of the standard must of course have been made first so that it is ready for use when the unknown is ready for the color comparison. The height of the standard usually $20 \mathrm{~mm}$., divided by the reading of the unknown and multiplied by 6 gives the "total creatinine" in $\mathrm{mg}$. per 100 c.c. blood.

In the case of uremic bloods containing large amounts of creatinine 1, 2, or 3 c.c. of blood filtrate, plus water enough to make approximately 5 c.c. are substituted for 5 c.c. of the undiluted filtrate. The normal value for "total creatinine" given by this method is about $6 \mathrm{mg}$. per 100 c.c. of blood.

Determination of Uric Acid in Blood. (Folin: Jour. Biol. Chem., xxxviii, 81, 1919).--Solutions Required for Uric Acid DETERMinations.

1. The standard uric acid sulphite solution. In a 500 c.c. flask dissolve exactly $1 \mathrm{~g}$. of uric acid in 150 c.c. of water by the help of $0.5 \mathrm{~g}$. lithium carbonate. Dilute to 500 c.c. and mix. Transfer 50 c.c. to a liter flask; add 500 c.c. of $20 \%$ sodium sulphite solution; dilute to volume and mix. Transfer to small kottles (cap. 200 c.c.) and stopper tightly. This standard uric acid solution keeps almost indefinitely in unopened bottles, because the sulphite prevents the spontaneous oxidation of the uric acid. In used bottles the standard usually remains good for 2-3 months.

2. A $10 \%$ sodium sulphite solution.

3. A $5 \%$ sodium cyanid solution, to be added from a buret.

4. A $10 \%$ solution of sodium chlorid in 0.1 normal hydrochloric acid.

5. The uric acid reagent prepared according to Folin and Denis. Introduce into a flask:

750 c.c. of water,

$100 \mathrm{~g}$. of sodium tungstate,

80 c.c. of phosphoric acid $\left(85 \% \mathrm{H}_{3} \mathrm{PO}_{4}\right)$. 
Partly close the mouth of the flask with a funnel and small watch glass and boil gently for two hours. Dilute to a liter.

A still stronger reagent is obtained by heating the sodium tungstate $(100 \mathrm{gm}$.$) and the phosphoric acid (80$ c.c.) plus water (700 c.c.) for 24 hours, instead of 2 hours; but the advantage gained, about $20 \%$, is not needed. Dilute the solution to 1 liter.

6. A solution of $5 \%$ silver lactate in $5 \%$ lactic acid.

To 10 c.c. of blood filtrate in each of two centrifuge tubes add 2 c.c. of a $5 \%$ solution of silver lactate in $5 \%$ lactic acid, and stir with a very fine glass rod. Centrifuge; add a drop of silver lactate to the supernatant solution, which should be almost perfectly clear and should not become turbid when the last drop of silver solution is added. Remove the supernatant liquid by decantation as completely as possible. Add to each tube 1 e.c. of a solution of $10 \%$ sodium chlorid in 0.1 normal hydrochloric acid and stir thoroughly with the glass rod. Then add 5 to 6 c.c. of water, stir again, and centrifuge once more. By this chlorid treatment the uric acid is set free from the preripitate. 'Transfer' the two supernatant liquids by decantation to a 25 c.c. volumetric flask. Add 1 c.c. of a $10 \%$ solution of sodium sulphite, 0.5 c.c. of a $5 \%$ solution of sodium eyanid, and 3 c.c. of a $20 \%$ solution of sodium carbonate. Prepare simultaneously two standard uric acid solutions as follows:

Transfer to one 50 c.c. volumetric flask 1 c.c. and to another 50 c.c. flask 2 c.c. of the standard uric acid sulphite solution described above. To the first flask add also 1 e.c. of $10 \%$ sodium sulphite solution. Then add to each flask 4 c.c. of the acidified sodium chlorid solution, 1 c.c. of the sodium cyanid solution, and 6 c.c. of the sodium carbonate solution. Dilute with water to about 45 c.c. When the two standard solutions and the unknown have been prepared as described they are ready for the addition of the uric acid reagent. Add 0.5 c.c. of this reagent to the unknown and 1 c.c. to each of the standards, and mix. Let stand for 10 minutes, fill to the mark with water, mix, and inake the color comparison. 
Calculation.-In connection with the calculation it is to be noted (a) that the blood filtrate taken corresponds to 2 c.c. of blood, (b) that the standard is diluted to twice the volume of the unknown, and (c) that the standard used contains 0.1 or 0.2 mg. of uric acid. The blood filtrate from blood containing 2.5 mg. of uric acid will be just equal in color to the weaker standard. Twenty times 2.5 divided by the reading of the unknown gives, therefore the uric acid content of the blood when the weaker standard is set at $20 \mathrm{~mm}$.

The two standards recommended were adopted on the basis of the experience gained from the analysis of more than 150 different samples of human blood. The uric acid may sink to as low as $1 \mathrm{mg}$. of uric acid per 100 c.c. of blood. It seems hardly worth while to prepare a third and weaker standard regularly in order to provide for such low acid values. A standard corresponding to the color obtained from $1.25 \mathrm{mg}$. of uric acid per 100 c.c. of blood can be prepared within a couple of minutes as follows: Transfer 1 c.c. of $10 \%$ sulphite solution, 3 c.c. of $20 \%$ sodium earbonate, 2 c.c. of the acidified sodium chlorid, 0.5 c.c. of the sodium cyanid solution, and 25 c.c. of the weaker one of the two regular standard solutions already on liand. Dilute to 50 c.c. and mix. Or, simply add 5 c.c. of $20 \%$ sodium carbonate to 25 c.c. of the regular weaker standard, and dilute to 50 c.c.

If a low uric acid value is expected, an alternate procedure is to dilute the unknown to a final volume of 10 c.c. with corresponding reduction in the amount of the reagents used.

Special attention should perhaps be called to one small yet essential variation in the process for developing the blue uric acid color, a variation made necessary by the use of sodium sulphite. The uric acid reagent must invariably be added after, and not before, the addition of the sodium carbonate, because in acid solution the sulphite will itself give a blue color with phosphotungstic acid.

Blood Sugar. (Folin and Wu: Jour. Biol. Chem., xli, 368, March, 1920.) - Sugar Reagent.-Transfer to a liter beaker 35 
gm. of molybdic acid and $5 \mathrm{gm}$. of sodium tungstate. Add 200 c.c. of $10 \%$ sodium hydroxide and 200 c.c. of water. Boil vigorously for 20 to 40 minutes so as to remove nearly the whole of the ammonia present in the molybdic acid. Cool, dilute to about 350 c.c. and add 125 c.c. of concentrated (85\%) phosphoric acid. Dilute to 500 c.c.

It will be seen that the preparation of this reagent is much simpler than the preparation of the phenol reagent. The solution has none of the ycllow color of the phenol reagent, yet gives an intense blue color with cuprous oxide. Since the reaction takes place in acid solution the blue color of the alkaline copper tartrate is also eliminated. The sodium tungstate contained in this reagent is added because there is sodium tungstate in our blood filtrates, and tungstates modify somewhat the shade of blue obtained in the reaction.

Alkatine Copper Solution.-The alkaline copper solution has not been changed. Dissolve $40 \mathrm{gm}$. of pure anhydrous sodium carbonate in about 400 c.c. of water and transfer to a liter flask. Add $7.5 \mathrm{gm}$. of tartaric acid, and when the latter has dissolved add $4.5 \mathrm{gm}$. of crystallized copper sulfate. Mix and make up to a volume of 1 liter. If the chemicals used are not pure a sediment of cuprous oxide may form in the course of 1 or 2 weeks. If this should happen, remove the clear supernatant reagent with a siphon, or filter through a good quality filter paper. Our reagent seems to keep indefinitely. To test for the absence of cuprous copper in the solution, transfer 2 c.c. to a test tube and add 2 c.c. of the molybdate phosphate solution; the deep blue color of the copper should almost completely vanish. In order to forestall improper use of this reagent attention should be called to the fact that it contains extremely little alkali, 2 c.c. by titration (using the fading of the blue copper tartrate color as indicator), requiring only about 1.4 c.c. of normal acid.

Standard Sugar Solution.-Three standard sugar solutions should be on hand: (1) a stock solution, $1 \%$ dextrose or invert sugar, preserved with xylene or toluene; $(2)$ a solution contain- 
ing $1 \mathrm{mg}$. of sugar per 10 c.c (5 c.c. of the stock solution diluted to 500 c.c.) ; (3) a solution containing $2 \mathrm{mg}$. of sugar per 10 c.c. (5 c.c. of the stock solution diluted to 250 c.c.). The invert sugar solution has the advantage that it can be easily prepared from cane sugar, which is pure. The keeping quality of such solutions should be less good than those made from glucose, but we have encountered no trouble on that score. When good quality glucose is available, it is, of course, the one to use. The diluted solutions should be preserved with a little added toluene or xylene; it is probably better not to depend on such diluted solutions to keep for more than a month, but the stock solution should keep indefinitely.

Determination.-The blood sugar determination is now made as follows:

Transfer 2 c.c. of the tungstic acid blood filtrate to a blood sugar test tube, and to two other similar test tubes (graduated at 25 c.c.) add 2 c.c. of standard sugar solution containing respectively 0.2 and $0.4 \mathrm{mg}$. of dextrose. To each tube add 2 c.c. of the alkaline copper solution. The surface of the mixtures must now have reached the constricted part of the tube. If the bulb of the tube is too large for the volume (4 c.c.) a little, but not more than 0.5 c.c. of a diluted $(1: 1)$ alkaline copper solution may be added. If this does not suffice to bring the contents to the narrow part, the tube should be discarded. Test tubes having so small a capacity that 4 c.c. fills them above the neck should also be discarded. Transfer the tubes to a boiling water bath and heat for 6 minutes. Then transfer them to a cold water bath and let cool, without shaking, for 2 or 3 minutes. Add to each test tube 2 c.c. of the molybdate phosphate solution. The cuprous oxide dissolves rather slowly if the amount is large but the whole, up to the amount given by 0.8 mg. of dextrose, dissolves usually within 2 minutes. When the cuprous oxide is dissolved dilute the resulting blue solutions to the 25 c.c. mark, insert a rubber stopper, and mix. It is essential that adequate attention be given to this mixing be- 
cause the greater part of the blue color is formed in the bulb of the tube. Make the reading in a Colorimeter as usual.

Method for the Determination of Chlorids in Blood Plasma. (Rappleye: Jour. Biol. Chem., 1918, xxxii, 509).-In this method the principle used in the Volhard Method for the determination of chlorids in urine is employed for the estimation of the minute amounts of sodium chlorid found in blood plasma.

The following solutions are required:

\section{Solution I}

Silver Nitrate, $7.2653 \mathrm{gms}$.

Nitric Acid (concentrated), 250 c.e.

Saturated Solution of Iron-Ammonium-Alum, 50 c.c.

Distilled water to make 1000 c.c.

\section{Solution II}

Potassium sulphocyanate in distilled water of such strength that 25 c.c. is exactly equivalent to 5 c.c. of the silver nitrate solution. Each c.c. of the silver nitrate solution is exactly equivalent to $2.5 \mathrm{mg}$. of sodium chlorid and each c.c. of the potassium sulphocyanate is equivalent to $0.5 \mathrm{mg}$. of sodium chlorid.

\section{Procedure}

Place 2 c.c. of eitrated plasma (oxalated plasma cannot be used on account of the peor end point) in a 50 c.c. volumetric flask containing. 30 c.c. distilled water. Add 10 c.c. of Solution I and make to mark. After being mixed the liquid is allowed to stand for 5 to 10 minutes and is then filtered through a dry filter paper free from chlorids. Twenty-five c.c. of the filtrate is then titrated with Solution II.

To calculate the result subtract the number of c.c. of Solution II used in the titration from 25 and multiply by 50 . This gives the number of milligrams of sodium chlorid present in 100 c.c. of blood plasma. 


\section{CHAPTER VII}

\section{SALIVARY DIGESTION}

Rinse the mouth with distilled water and collect some saliva by chewing a small piece of paraffin. Filter and note color, and transparency. The saliva obtained in this way is a mixture of the secretions of the three classes of salivary glands. Its composition varies with the nature of the stimulus causing secretion.

Note the turbidity which increases on standing as a result of the precipitation of calcium carbonate. Saliva usually contains epithelial cells or cell debris. Test the chemical reaction of the saliva with phenolphthalein, litmus, and methyl orange. Saliva is usually slightly alkaline, but not sufficiently so to turn phenolphthalein red. This indicator turns color when the hydrogen-ion concentration is $\mathrm{N} \times 10^{-9}$. Saliva usually is alkaline to litmus, which changes color at a hydrogen-ion concentration of $\mathrm{N} \times 10^{-7}$ or about the true neutral point. The reaction of the saliva thus lies usually between a hydrogen-ion concentration of $\mathrm{N} \times 10^{-7}$ and $\mathrm{N} \times 10^{-9}$. In case the saliva reacts acid to litmus, it still will be alkaline to methyl orange, which changes color at a hydrogen-ion concentration of $\mathrm{N} \times 10^{-4}$.

\section{Composition.-}

Mucin.-Recall the preparation and properties of mucin studied in the work on proteins.

Other Proteins.-The saliva contains, in addition to mucin, small traces of other proteins, the presence of which may be demonstrated after the removal of mucin. Precipitate mucin by adding acetic acid, filter and apply the biuret test to the filtrate. 
1. Test saliva for chlorides and for sulphates.

2. For sulphocyanates as follows: To 2 c.c. of saliva in a small evaporating dish, add a few drops of dilute $\mathrm{HCl}$ and then a drop or two of dilute ferric chloride. A reddish color indicates the presence of sulphocyanate. Compare the color with that produced by adding a similar amount of ferric chloride to distilled water and $\mathrm{HCl}$ in an evaporating dish. Note that the color is a pure greenish yellow with no suggestion of pink. To this control add a few drops of dilute potassium sulphocyanate. A comparison of this color with that obtained with saliva will indicate the very small amount of sulphocyanate in the latter fluid.

3. Phosphates and carbonates also occur in the saliva and may be detected by appropriate tests.

Ptyalin.-This ferment acts on starch, breaking it down into dextrins, maltose, and isomaltose. It may be isolated by precipitation with alcohol. Its action may be studied, however, without isolation. Saliva is said to contain an erepsin, which acts on peptids. Its action is unimportant, however. Saliva contains no lipase.

\section{Digestive Action.-}

4. On Starch.-Prepare a starch mucilage by mixing about one gram of starch with 25 c.c. cold distilled water. Add about 75 c.c. of boiling water and boil for 15 minutes, stirring occasionally and adding water to replace loss by distillation. Cool, and use this mucilage in the following experiments.

\section{Arrange 6 test tubes as follows:}

a. 6 c.c. distilled water and a few grains of raw starch.

b. 5 c.c. water, 1 c.c. saliva and a few grains of raw starch.

c. 1 c.c. saliva and 5 c.c. starch mucilage.

d. 1 c.c. saliva and 5 c.c. starch mucilage and 3 drops $10 \%$ $\mathrm{NaOH}$. $\mathrm{HCl}$.

e. 1 c.c. saliva and 5 c.c. starch mucilage and 5 drops $10 \%$ 
f. Boil a fevi e.c. of saliva thoroughly for three or four minutes, cool, and add 1 c.c. to 5 c.c. starch mucilage.

Put all six tubes in a beaker of water warmed to $40^{\circ}$ for 15 minutes. Test each solution for sugar with Benedict's solution and for starch with iodine. A positive Benedict indicates that a portion of the starch has been broken down into a reducing sugar (maltose and isomaltose). If the iodine test is still blue, some starch remains. If it is reddish, the solution contains dextrine. If iodine gives no color, the material has all passed to the achroodextrin stage or beyond.

Record the results of your observations and draw conclusions as to the conditions under which ptyalin will digest starch.

5. Effect of cooling.-Place a test tube containing saliva in a freezing mixture for a few minutes. Cool 5 c.c. of starch mucilage in a separate test tube. Add 1 c.c. of the saliva to 5 c.c. starch mucilage and leave in a freezing mixture for 15 minutes. In a portion, test for sugar with Benedict's solution. If the solutions have been well cooled, little or no digestion will have taken place,-at least much less than at body temperature.

The most favorable reaction for ptyalin digestion is a very weak acidity (Hydrogen-ion concentration $\mathrm{N} \times 10^{-5.3}$ ). A hydrogen-ion concentration of $\mathrm{N} \times 10^{-4}$ is sufficient acid to stop its action. It will act in a weakly alkaline solution such as the saliva, however. The acidity of the contents of tube number 5 above should be sufficient to destroy ptyalin.

6. On Cane Sugar.-Add saliva to a few c.c. of cane sugar solution. Digest at $40^{\circ}$ for 15 minutes and test with Benedict's solution. Does saliva contain an invertase?

On Proteins and Fats.-Proteins and fats are not digested by saliva, since this secretion contains no proteolytic or lipolytic enzyme. The erepsin mentioned above is believed to be unimportant, and acts not on proteins, but on peptids.

7. Progress of Digestion by Ptyalin.-To 50 c.c. of starch mucilage add 10 c.c. of saliva. Digest at $40^{\circ}$ and at intervals of a minute or two, remove a few drops and test with iodine. From 
the results of the iodine test, record the time required in each stage of the digestion of starch to achroodextrin under the above conditions.

Products of Ptyalin Digestion.-The dextrins and maltose produced by the action of ptyalin may be isolated by precipitating the former with alcohol. In the remaining solution maltose may be identified by its reaction with Benedict's solution and the preparation of its osazone with phenylhydrazine. 


\section{CHAPTER VIII}

\section{GASTRIC DIGESTION}

\section{Preparation of Artificial Gastric Juice}

Strip the mucous membrane from the stomach of a pig, and cut into small pieces. Cut about $1 / 5$ of the mucous membrane into very small pieces, place in a small beaker and cover with glycerine. Allow to stand at room temperature for 24 hours, stirring occasionally. Decant or draw off with a pipette the resulting glycerine solution of pepsinogen. This solution will keep for a long time. Use it for the digestion experiments in this chapter. To the remaining $4 / 5$ of the gastric mucosa, add 300 c.c. of $0.35 \% \mathrm{HCl}$ (3 c.c. of concentrated $\mathrm{HCl}$ diluted to 300 c.c.), a few c.c. of chloroform and set in the incubator at $40^{\circ}$ for at least 48 hours, or longer if possible. The extracted pepsin will digest the protein of the mucosa, and the mixture may be studied for the products of peptic digestion as described below.

\section{Composition of Gastric Juice.-}

Natural gastric juice contains small amounts of mucin and other proteins.

Inorganic salts, - $\mathrm{NaCl}$, earthy phosphates, etc.

Acids, chiefly $\mathrm{HCl}$. The acidity of the stomach contents may be due to several factors, e. g., free $\mathrm{HCl}$, organic acids, acid combined with protein, and acid salts. The sum of these is known as the total acidity. It may be determined by titrating with $\mathrm{N} / 10 \mathrm{NaOH}$, using phenolphthalein as indicator. Until recently the individual factors which go to make up total acidity have been estimated by the use of various indicators sensitive to different concentrations of hydrogen-ions. These methods have been shown to be subject to great inaccuracy. 
If it is desired to know the hydrogen-ion concentration of gastric contents, the determination may be made by electrolytic methods, or approximately by the use of certain series of indicators.

For most clinical work the only data required are total acidity as determined by titration, using phenolphthalein as indicator, and free hydrochloric acid as determined by titration using Toepfer's reagent as indicator or as determined with Guenzberg's reagent.

Study the following indicators, by observing their color in faintly acid and faintly alkaline solution.

1. Congo red gives a blue color with free $\mathrm{HCl}$, violet with an organic acid and brown with combined $\mathrm{HCl}$. Add a few drops of congo red to $0.2 \% \mathrm{HCl}$ and to $0.5 \%$ acetic acid. Neutralize one with $\mathrm{NaOH}$. Add 2-3 drops of congo to a few c.c. of your hydrochloric acid extract of pig's stomach.

2. Guenzberg's reagent (2 g. phloroglucin, 1 g. vanillin, 100 c.c. alcohol) produces a purplish red color on evaporating with free $\mathrm{HCl}$. Place 2 drops of the reagent in an evaporating dish. Evaporate to dryness on the water bath. A yellow spot results. Add 2 drops of $0.2 \% \mathrm{HCl}$ and replace on the water bath. Observe the red spot. Repeat with 2 drops of your $\mathrm{HCl}$ extract of stomach mucosa. Repeat with 2 drops of lactic acid. Guenzberg's test may be made roughly quantitative by diluting the acid solution until it will just respond with a faint positive reaction. At this dilution the $\mathrm{HCl}$ is about 1/2500 normal.

3. Uffelmann's Test for Lactic Acid.-In each of 3 test tubes place 5 c.c. of $1 \%$ phenol and two or three drops of ferric chloride solution. The resulting amethyst liquid is Uffelmann's reagent. To one tube add 2 e.c. dilute lactic acid. To the second 2 c.c. $0.2 \% \mathrm{HCl}$ and to the third 2 c.c. acetic.

4. To a small amount of $0.2 \% \mathrm{HCl}$ add Toepfer's reagent (dimethyl-amino-azobenzene). Make slightly alkaline and observe the change in color.

5. Determination of Acidity of Gastric Contents.-This may 
be accomplished as follows : The subject eats only a cup of clear tea and two pieces of dry toast for breakfast. One and one-half hours after breakfast, his stomach contents are pumped out by means of a stomach pump. Ten c.c. portions of this liquid are titrated with $\mathrm{N} / 10$ alkali using phenolphthalein as indicator. This gives total acidity.

Free hydrochloric acid may be determined by titration with N/10 alkali using Toepfer's reagent as indicator, or by the Guenzberg reagent method described above. The gastric contents may be tested for blood by the guaiac test (as performed in the study of hemoglobin).

Ferments. The gastric juice contains two or perhaps three ferments: pepsin, rennin and lipase.

\section{Digestive Action of Gastric Juice.-}

ON Proteins.-A satisfactory method for the study of the digestive action of gastric ferments on proteins is the use of Mett's tubes. Mett's method consists in suspending in a digestive mixture short glass tubes filled with coagulated egg albumin. After incubating, the length of the column of albumin which has been digested is observed, and the activity of the digestion mixture judged accordingly.

\section{Preparation of Mett's Tubes.-}

White of egg is beaten to break the reticulum, strained through linen or muslin, and allowed to stand until free from air bubbles. The egg white is then drawn up into lengths of glass tubing having an inner bore of $1-3 \mathrm{~mm}$. The tubing is then placed on a wire gauze so arranged that it can be lowered into the inner compartment of a double boiler. The water in the double boiler is heated until that in the inner compartment reaches a temperature of $85^{\circ} \mathrm{C}$. The gauze is then lowered into the water and allowed to remain until quite cold. The tubes of coagulated albumin may be preserved by covering the ends with shellac. For use, the tubes are eut into $2 \mathrm{~cm}$. lengths, breaking the tube sharply to get an even edge of albumin. 
7. Arrange six test tubes as follows, numbering them Nos. 1 to 6 , and labelling with your name.

1. 5 c.c. distilled water.

2. 5 c.c. $0.2 \%$ hydrochloric acid.

3. 5 c.c. water +10 drops glycerine extract of pig's stomach prepared above.

4. 5 c.c. $0.2 \% \mathrm{HCl}+10$ drops glycerine extract.

5. 5 c.c. $0.2 \% \mathrm{HCl}+10$ drops glycerine extract, boil thoroughly 3 or 4 minutes and cool.

6. 5 c.c. $0.3 \%$ sodium earbonate +10 drops glycerine extract.

In each tube suspend a Mett tube by means of a thread supported by a match. Make sure that the Mett tube hangs so that it does not touch the bottom of the test tube. The digestion liquid must have free access to the egg white. Place in the incubator until the next period. After a minimum time of 24 hours, compare the amounts of digestion in the different tubes and record results. Why does not No. 3 digest?

8. ON MiLk.-The ferment rennin, by some investigators considered to be identical with pepsin, causes the clotting of milk due to precipitation of casein. Calcium salts are necessary for this process. Prepare two test tubes each containing 5 c.c. of milk.

1. To one add 3 c.c. ammonium oxalate.

2. To the second add 3 c.c. distilled water.

As the rennin in the glycerine extract is very likely to have become inactive, powder a rennin tablet and add half of the powder to cach tube.

Place the tubes in water at $40^{\circ}$ for 20 minutes. What happens? Explain. To the oxalate tube add 3-4 drops of concentrated calcium chloride solution. Explain.

Gastric juice contains also a lipase, but its action is not extensive.

9. Products of Gastric: Digestion.-When the hydrochloric acid stomach mucosa mixture has remained in the incubator for 2 or 3 days or longer, remove it, boil, filter and neutralize the 
whole of the filtrate. Any metaprotein will precipitate. Filter if necessary and saturate with solid ammonium sulphate. Proteoses precipitate. Filter and test for peptones. Gastric digestion takes the proteins no lower than peptones.

\section{Motor Power of the Stomach.-}

10. Take by mouth a capsule containing 0.1 gm. iodoform. This is not decomposed until it reaches the intestine. Test saliva for iodine by spitting upon starch paper and dropping one drop of conc. nitric acid upon the spot. A positive test appears in from 1 to $1 \frac{1}{2}$ hours.

\section{Rate of Absorption From the Stomach.-}

11. Take by mouth $0.2 \mathrm{~g}$. potassium indine in a capsule. Test saliva at minute intervals for iodine as in 10. A positive test appears in from 10 to 15 minutes.

12. Take by mouth a capsule containing $1 \mathrm{gm}$. of salol. In the intestine this is broken up into phenol and salicylic acid. A violet color imparted to the urine by a few drops of ferric chloride indicates salicylic acid. A test should appear in from 1 to $1 \frac{1}{2}$ hours. 


\section{CHAPTER IX}

\section{PANCREATIC DIGESTION.-BILE}

\section{Pancreatic Juice}

A solution of pancreatic ferments may be prepared by extracting a pig's pancreas with glycerine or with water containing chloroform. For laboratory purposes it is, however, more convenient to use a solution of commercial pancreatic powder.

\section{Composition of Pancreatic Juice.-}

Natural pancreatic juice contains small amounts of proteins and other organic substances.

Inorganic salts, chiefly sodium carbonate.

Ferments. The active pancreatic juice contains three important enzymes : trypsin, amylase, and lipase.

\section{Digestive Action.-}

On proteins.

1. Prepare four test tubes as follows:

a. 5 c.c. neutral pancreatic solution.

d. 5 c.c. neutral pancreatic solution +2 drops saturated sodium carbonate solution. This gives a concentration of about $0.2 \%$ sodium carbonate.

c. 5 c.c. neutral pancreatic solution +3 to 4 drops of $10 \%$ HCl. This gives a concentration of about $0.2 \%$ acid.

d. 5 c.c. neutral pancreatic solution +2 drops saturated sodium carbonate solution, boil thoroughly 3 or 4 minutes and cool.

To each tube add a Mett tube as described under "Gastric Digestion" and incubate until the next period.

Examine the tubes and tabulate results.

Trypsin acts best in a slightly alkaline solution. It also will 
act in neutral or even faintly acid solution. It is, of course, destroyed by boiling.

2. On Fats.-Pancreatic juice contains a lipase. To 5 c.c. milk add 3 or 4 drops of litmus or lacmoid solution, and enough sodium carbonate to produce a blue color (no more). Add the amount of pancreatic powder which can be taken up on a knife point. Keep at body temperature for some time. The color turns pink, since fats are split, fatty acids set free, and the reaction becomes acid. If the test is allowed to stand too long, an acid reaction will result from the souring of the milk (production of lactic acid from lactose). The bile greatly favors the digestion of fats by pancreatic juice.

On Starch.-The pancreatic juice contains an amylase, which soon becomes inactive in artificial preparations.

Products of Pancreatic Digestion of Proteins.

Recall the results of your work on products of pancreatic digestion under amino acids.

\section{Intestinal Juice}

The succus entericus or intestinal juice plays an important rôle in digestion. For a description of the intestinal ferments and their action, the student is referred to the discussion of this subject in the text.

\section{Bile}

The bile is secreted by the liver into the gall bladder and thence delivered to the intestine. Bile plays an important part in digestion. Note the green or yellow color. Test the reaction with litmus. It usually is neutral or slightly alkaline.

\section{Composition.-}

Inorganic Material.- The bile contains various inorganic substances, among them the phosphates of calcium, magnesium and iron; sodium and potassium, both in the form of chlorides and combined with bile acids to form bile salts; sulphur and 
phosphorus in organic combination, and various substances. Inorganic sulphates are absent or present only in traces.

Bile Acids in the Form of Alkali Salts.

3. Pettenkofer's test for bile salts. To 5 c.c. of bile add a few grains of cane sugar or a few drops of cane sugar solution. With a pipette (caution: do not draw acid into the mouth), add concentrated $\mathrm{H}_{2} \mathrm{SO}_{4}$ to form a layer at the bottom, or allow 2-3 c.c. of acid to run down the side of the tube. Note the red ring.

4. Extraction of bile salts. Bile salts may be prepared by heating bile, charcoal (enough to make the mixture fairly thick) and 5 volumes of alcohol on the water bath for 20 minutes, replacing alcohol lost by evaporation. Filter off the liquid and add ether in excess. Bile salts are precipitated.

Bile Pigments.

5. Gmelin's test. To about 5 c.c. of concentrated $\mathrm{HNO}_{3}$ in a test tube, add 2-3 c.c. of diluted bile, carefully, so that the two liquids do not mix. Note the colored rings.

Mucin and Pseudomucin. Bile is said, by some authorities to contain mucin. On the addition of 5 volumes of alcohol to bile a precipitate is formed. This is called pseudomucin, and is believed by some to be a phosphoprotein.

In addition to the above constituents, bile also contains small amounts of fats, lecithin, phosphates, cholesterol, etc.

6. Effect of Bile on Surface Tension.-Fill one small beaker with distilled water. To a second beaker, add bile diluted with 4 parts of water. Sprinkle a few grains of powdered sulphur over each. In the beaker containing bile, the sulphur sinks to the bottom. The surface tension of the water has been reduced so that the surface film is no longer able to support the, sulphur grains.

Biliary Calculi, or Gall Stones, are of four kinds:

a. Those made up of calcium, iron or copper combined with bile pigments.

b. Cholesterin calculi.

c. Calculi of calcium phosphate and carbonate.

d. Calculi of combinations of the above. 
Analysis of gall stones.-Extract gall stone powder with a small volume of ether. Filter through a dry filter, allow ether to evaporate and observe the residue of cholesterin (1). The material which did not dissolve in ether consists mainly of inorganic substances and bile pigments. Dissolve out the inorganic material by adding a small amount of $10 \% \mathrm{HCl}$. (See 9a below.) The filtrate contains inorganic material (9) and the residue consists mainly of bile pigments (12).

7. Examine under a microscope the crystals left on evaporating the ether extract. If the crystals are not well formed, dissolve a portion of them in a very small quantity of alcohol and allow to evaporate slowly. Draw crystals. They should be large, colorless plates.

8. To a few cholesterin crystals add concentrated $\mathrm{H}_{2} \mathrm{SO}_{4}$ and note the red color.

9. Inorganic Material.-Analyze the hydrochloric acid extract obtained above as follows:

Carbonates. If carbonates were present, an evolution of $\mathrm{CO}_{2}$ will have been observed on the addition of $10 \% \mathrm{HCl}$ above.

10. Phosphates.-Evaporate a portion of the $\mathrm{HCl}$ extract to dryness on the water bath, take up with a few drops of concentrated $\mathrm{HNO}_{3}$, dilute with a few cubic centimeters of water and add ammonium molybdate in excess. Observe the yellow crystals of ammonium phospho-molybdate.

11. Calcium.-Evaporate the remainder of the $\mathrm{HCl}$ extract to dryness, take up with a few cubic centimeters of $10 \%$ acetic acid and add ammonium oxalate. Calcium oxalate precipitates.

12. Bile Pigments.-The residue insoluble in $10 \% \mathrm{HCl}$ is extracted several times with chloroform. A yellow color indicates bilirubin. If time permits, filter the chloroform extract, evaporate to dryness and test for bile pigments. The residue insoluble in chloroform may be extracted with a few cubic centimeters of hot alcohol, filtered, evaporated and the resulting residue tested for biliverdin. 


\section{CHAPTER X}

\section{URINE}

\section{Qualitative Study}

For the qualitative study of urine each student will need 2 liters of urine. (3 liters, if purine bases are to be included.) The urine should be preserved by the addition of a $5 \%$ solution of thymol in chloroform, about 5 c.c. per liter of urine. This preservative should be placed in the flask before the urine is collected, as otherwise the urine may decompose.

\section{Inorganic Constituents.-}

1. Chlorides.-Acidify about 10 c.c. of urine with 2-3 drops conc. nitric acid and add a drop of silver nitrate. If chlorides are present in normal quantity, a solid clump of silver chloride will sink to the bottom. If but small amounts of chlorides are present, the urine becomes only cloudy. If an attempt be made to confirm chlorides by dissolving the precipitate in ammonia, a heavy flocculent precipitate of earthy phosphate will be thrown down. Such a precipitate might be filtered off however, and the dissolved chlorides reprecipitated by adding nitric acid.

Phosphates.-There are two general classes of phosphates in urine,-alkali, i.e., sodium and potassium, and earthy, i.e., calcium and magnesium. These phosphates are present both as mono- and di-hydrogen salts, e.g., $\mathrm{Na}_{2} \mathrm{HPO}_{4}$ and $\mathrm{NaH}_{2} \mathrm{PO}_{4}$. There also is phosphorus in urine which is not detected in the usual precipitation tests. It is in organic combination and may be detected only after fusion with an oxidizing agent.

2. Earthy Phosphates. - To about 10 c.c. urine, add ammonia to alkalinity and warm. A flocculent precipitate is earthy phosphates. The alkali phosphates remain in solution.

3. Filter off the precipitated earthy phosphates and add 
magnesia mixture to the filtrate. Observe a precipitate due to alkali phosphates. The fairly soluble alkali phosphates are precipitated as magnesium ammonium phosphate.

SuLPHATES.-Sulphur is present in three forms: Inorganic sulphates, ethereal sulphates, and unoxidized sulphur in organic combination. To determine unoxidized sulphur, it is necessary first to fuse with an oxidizing agent.

4. Inorganic S'ulphates.-To about 10 c.c. of urine add 2-3 drops of concentrated $\mathrm{HCl}$ and barium chloride in excess. The inorganic sulphates are precipitated as barium sulphate, which will settle to the bottom as a whitish layer on standing.

5. Ethereal Sulphates.-Filter off the precipitated barium sulphate. If the filtrate does not come through clear, mix with it a small amount of bismuth subnitrate and filter repeatedly through the same filter. The bismuth subnitrate. stops the larger pores of the filter paper, thus helping to keep back the fine particles of barium sulphate. To the clear filtrate add about 2 c.c. conc. $\mathrm{HCl}$ and boil for some minutes. Ethereal sulphates are split up in this process, and since there is still excess of barium chloride, a second "crop" of barium sulphate is obtained. As the amount is small, it may not be seen until the test has stood for a few minutes, when the precipitate will form a film or layer of deposit at the bottom of the tube.

6. Carbonatrs.-Evaporate 10 c.c. urine (Hood) to dryness on the water bath. Add $10 \% \mathrm{HCl}$. Observe the evolution of carbon dioxide from the decomposed carbonates.

7. Ammonia.-To about 10 c.c. urine in a test tube, add sodium carbonate until alkaline. Moisten a piece of red litmus paper with distilled water and hang in the mouth of the tube. What happens?

In addition to the above inorganic materials, urine contains varying amounts of sodium, potassium, calcium, magnesium, iron, fluorine, nitrates, silicates, traces of hydrogen peroxide, and dissolved gases, e. g., $\mathrm{CO}_{2}, \mathrm{~N}_{2}$, and $\mathrm{O}_{2}$, besides a possible number of casual constituents which may be taken in the food and gotten rid of by way of the kidneys. 


\section{Organic Constituents.-}

UREA.

8. Preparation from Urine.-Evaporate 500 c.c. of urine on the water bath to the consistency of a thin syrup. Cool and add twice the volume of nitric acid (50\%). Allow to stand in a cool place over night. Filter off the crystals (dry between filter papers), remove crystals from the filter, and dissolve them in a small amount of hot water. Add a little potassium permanganate solution to this urea nitrate solution, stirring constantly, until the nitrate solution is nearly colorless. Bring the solution to a boil, and add first solid sodium carbonate and then solid barium carbonate until $\mathrm{CO}_{2}$ ceases to come off, and the solution is neutral. Evaporate over the water bath to dryness, powder the residue and extract it with $95 \%$ warm alcohol. Filter and set the filtrate aside to cool. Examine the crystals under the microscope.

\section{Reaction of Urea.}

9. Test solubility of urea in alcohol.

10. Heat a few crystals of urea gently in a dry test tube. Add a small volume of water and perform the biuret test. Explain.

11. Dissolve a few crystals of urea in a few drops of water. Add 2 drops of conc. $\mathrm{HNO}_{3}$. Observe the crystals of urea nitrate under the microscope, and draw. Repeat with oxalic acid and observe the urea oxalate.

12. Dissolve a few crystals of urea in a few cubic centimeters of water and add sodium hypobromite solution.

Note: Sodium hypobromite is prepared by adding bromine water to sodium hydrate solution.

Note effervescence. This is due to the liberation of free nitrogen in the decomposition of urea by the hypobromite.

\section{3-14. URIC ACID.}

To a liter of urine add 25 c.c. conc. hydrochloric acid. Allow to stand in the ice chest over night. Decant carefully from the reddish brown crystals and collect them on a small filter. 
Examine microscopically. The crystals are colored dark brown by urine pigment, and occur in a variety of forms, lozenge or "gunboat" shapes, thick rods, etc. These crystals are not pure but serve for a study of the reactions of uric acid.

Reactions of uric acid.

15. Solubility. Test solubility of urie acid in ammonia, sodium hydroxide, and concentrated sulphuric acid.

16. Murexid test. To a few crystals of uric acid in an evaporating dish add 2-3 drops cone. nitric acid and evaporate to dryness. Cool the dish and add a drop of dilute ammonia. Observe the red or purple spot. This test is given also by xanthine. To distinguish between the two substances again evaporate to dryness. The color disappears if the substance is uric acid. If it is xanthine, the red color persists.

17. Boil a few crystals of urie acid with a small amount of Fehling's solution. A slight reduction will occur, but it may become apparent only by allowing the cuprous oxide to settle to the bottom of the tube. This should occur inside of ten or fifteen minutes after the boiling ( $1 / 2$ minute) is stopped. This result should be kept in mind in testing supposed diabetic urines. The amount of uric acid in normal urine is so small, however, that it will not reduce Fehling's solution perceptibly.

Purine Bases.

18. Preparation (Salkowski Method).-To a liter of urine add 200 e.c. of magnesia mixture. Filter off the precipitate, add an excess of strong ammonia, and then 50-60 c.e. of $3 \%$ silver nitrate. Allow the mixture to stand for an hour and filter, washing the precipitate to remove excess of silver. Suspend the precipitate in 400 c.c. boiling water, acidify with a few drops of conc. HCl. Pass hydrogen sulphide into the hot solution as long as a precipitate forms. Boil the mixture a few minutes (Hood) to remove excess $\mathrm{H}_{2} \mathrm{~S}$ and filter off the precipitated silver sulphide. Evaporate the clear filtrate to dryness on the water bath. The residue contains uric acid and other purine bases. Extract the residue with boiling $3 \%$-sulphuric acid. This dissolves the xanthine bases and leaves the uric acid as a residue. Allow to stand 
over night, filter, and make the filtrate strongly alkaline with ammonia. Reprecipitate xanthine bases as above with silver nitrate. Filter, suspend the precipitate in boiling water acidulated with $\mathrm{HCl}$. Boil vigorously a few minutes and decompose the silver salts with $\mathrm{H}_{2} \mathrm{~S}$. Filter, and evaporate to dryness.

Reactions of Xanthine Bases.

19. Test solubility in water, alcohol, acids and alkalies.

20. Murexid test. Perform this test as described under uric acid. Note the method of distinguishing xanthine from uric acid.

\section{Creatinine.}

21. Preparation.-Creatinine may be prepared from urine by precipitation as the zine salt. Its reactions may be studied, however, without isolation from the urine.

\section{Reactions.}

22. Weyl's test. To about 10 c.c. of normal urine add 2-3 drops of sodium nitroprusside and then $2-3$ drops $10 \%$ sodium hydrate. A red color which soon fades to yellow shows the presence of creatinine. (Compare with Legal's test for acetone. See below.) On the addition of glacial acetic acid to this test, a pure solution of creatinine gives a grecnish color, which serves to distinguish it from acetone. In urine, however, this reaction with acetic acid is not easily recognized.

A second simple way to determine to which of these substances a positive test is due is to distill the urine. Acetone will pass over in the first few cubic centimeters of distillate, whereas creatinine will not distill.

23. Jaffe's test. To 10 c.c. of urine, add about 1 c.c. picric acid, and then 1-2 c.c. of $10 \%$ sodium hydrate. Observe the red color. This test serves as the basis for the quantitative estimation of creatinine. (See below).

24. Oxalic Acid.-To 200 c.c. urine in a beaker add 5 c.c. saturated solution of calcium chloride, acidify with acetic acid and set aside over night. Examine the sediment under the 
microscope and note the diagonally crossed crystals of calcium oxalate.

25. Urinary Indican.-Indican is usually, but not always, found in the urine. It is formed from the products of protein putrefaction in the intestine. To 15-20 c.c. urine in a test tube add 2 c.c. copper sulphate solution, 15 or 20 e.c. conc. $\mathrm{HCl}$ and 5 c.c. chloroform. Close the mouth of the tube with the thumb and shake cautiously (over the sink). Continue the shaking for several minutes. The "indican" is oxidized to indigo, which dissolves in the chloroform, giving it a blue color. The depth of color developed in the chloroform indicates roughly the amount of indican present.

Pigments.--Urochrome and uroerythrin are the most important urinary pigments.

Urochrome is a substance which gives the yellow color to urine.

Uroerythrin. This red pigment gives the deep reddish color to sediments of uric acid and urates.

In addition to the above constituents the urine contains small and varying amounts of various substances, e. g., hippuric acid, allantoin, aromatic oxyacids, compounds containing sulphur and many other substances.

\section{Collection and Preservation of a Specimen for Quantitative Analysis-General Properties}

As the composition of the urine voided at different times of the day varies considerably, it is customary to use for metabolism studies either a 3 -hour or a complete 24-hour sample. This may be obtained as follows: On rising in the morning empty the bladder and discard the urine voided. Then collect all urine voided during the day, and the first voiding on rising the following morning. This constitutes a complete 24-hour specimen. For the observations in this section and the quantitative analysis in the section following, a complete 24-hour specimen should be used, and all determinations made on the same specimen.

As some urinary constituents decompose on standing, it is necessary to add a preservative to the urine. In collecting a 24 
hour sample, 5 c.c. of $5 \%$ thymol in chloroform should be placed in the bottle intended for collection of the sample, before the urine is collected. Neglect of this precaution may lead to loss of the specimen through decomposition.

26. Volume. Measure in a large cylinder and record the volume of a 24 -hour sample.

27. Record the color, odor, transparency and presence or absence of a sediment.

Specific gravity. The specific gravity may be determined either gravimetrically or volumetrically. For most clinical work, the determination with an areometer or "urinometer" is sufficiently accurate.

28. Volumetrically.-Pour the urine into an areometer cylinder and determine the specific gravity with an areometer which registers 1.000 to 1.060. Make sure that the instrument floats free in the urine without touching the walls of the cylinder. As the instruments are standardized for a definite temperature (usually $15^{\circ} \mathrm{C}$.) it is necessary to correct for any variation in the temperature of the urine from that indicated on the instrument. For every $3^{\circ} \mathrm{C}$. variation a correction of 0.001 is added or subtracted from the reading, according as the urine is warmer or colder than the standard temperature. Observe the temperature of the urine and make any necessary correction as described above.

29. Gravimetrically.-If a gravimetric determination is desired, it may be made as follows: Carefully elean and dry a small weighing bottle and weigh quantitatively. From a pipette run into the weighing bottle 25 c.c. of distilled water at room temperature, stopper the bottle, and weigh.

Note: The pipette should be cleaned with cleaning fluid and then thoroughly rinsed, first with tap water and then once or twice with distilled water. If the liquid gathers in drops on the inside of the pipette after its contents have been allowed to flow out, the cleaning should be repeated. On emptying a perfectly clean pipette or burette, the instrument should be so clean that no drops remain hanging 
on the inside walls of the instrument; otherwise the amount delivered will not correspond to the amount engraved on the glass.

In filling a pipette, draw up the liquid into the stem, elose the top with the index finger, and allow the liquid to run out gradually, until the bottom of the meniscus is level with the graduation on the stem.

On emptying a pipette, allow the liquid to run out and drain for about one-half minute. Then touch the tip of the pipette to the surface of the liquid, or the side of the vessel. Do not blow out the small amount of liquid remaining in the tip. This is the method of emptying employed in the standardization of accurate registered pipettes. In the case of accurate work, if only ordinary pipettes or burettes are available, it is customary to standardize such instruments by weighing the amount of water they will deliver and ealculating the volume.

Clean and dry the weighing bottle and measure into it from the same pipette 25 c.c. of urine and weigh. Tabulate the weights of (a) weighing bottle, (b) weighing bottle + water, (c) weighing bottle + urine. Calculate the weights of water and urine. The specific gravity of the urine then may be determined by dividing the weight of urine by the weight of water. Since equal volumes of the two liquids have been weighed, it is unnecessary that the exact volume delivered by the pipette be known. Weighing the amount of water delivered by a pipette or burette serves to determine the accuracy of such an instrument, for a given weight of water corresponds to a definite volume at a fixed temperature. In standardizing volumetric apparatus it is necessary to correct for temperature. The accuracy of an areometer may be checked by trying it out on distilled water, not forgetting the correction for temperature.

30. Chemical Reaction. Test the chemical reaction of the urine with litmus paper. A 24 hour sample is usually acid, due to the presence of acid phosphates and both inorganic and organic acids. If the urine is alkaline, allow the litmus paper to dry on 
the water bath. If the blue color disappears, the alkaline reaction is due to ammonia. If it persists, to fixed alkalies.

For purposes of diagnosis of kidney disorders, it is sometimes advisable to determine both the freezing point and the electrical conductivity of the urine. The technique of these processes, is, however, beyond the scope of this course.

\section{Quantitative Analysis (Make All Determinations in Duplicate)}

Total Solids.-In order to make an accurate determination of total solids, it is necessary to evaporate a measured quantity of urine in a vacuum at room temperature, as the temperature of the boiling water bath causes decomposition of urea and loss of ammonia and $\mathrm{CO}_{2}$. By acidifying slightly with acetic acid it is possible partially to avoid this source of error. The residue is weighed quantitatively, and the total solids calculated from the result.

Determination of Acidity of Urine.

31. Preliminary Experiments.

Measure out 10 c.c. of potassium dihydrogen phosphate into each of 3 small beakers or Erlenmeyer flasks, and label 1, 2 and 3.

To No. 2 add 3-4 drops sat. $\mathrm{CaCl}_{2}$ soln.

To No. 3 add $3-4$ drops sat. $\mathrm{CaCl}_{2}$ sol. + about 10 c.c. $15 \%$ neutralized potassium oxalate solution. Titrate all 3 solutions with $\mathrm{N} / 10 \mathrm{NaOH}$, using phenolphthalein as indicator. Observe that the presence of ealcium increases the titration figure. The phosphates of the alkaline earths precipitate in alkaline solution. On adding oxalate, however, the disturbing influence of calcium is nullified, since it is precipitated as calcium oxalate.

32. Acidity of Urine.-Folin's Method.

Measure 25 c.c. of urine into an Erlenmeyer flask. Add 1-2 drops phenolphthalein and 10 c.c. neut. potassium oxalate solution. Shake vigorously for 1-2 minutes and titrate at once with $\mathrm{N} / 10 \mathrm{NaOH}$ to the first faint but permanent pink. From the value obtained calculate the total acidity of the 24 hour specimen in cubic centimeters of normal acid. 
33. Repeat the process described in 32 , omitting the addition of oxalate. Consider the result in connection with your observations in the preliminary experiments under section 31 .

\section{Total Nitrogen.-}

34. Determine the total nitrogen in 5 c.c. of urine by the Kjeldahl method and calculate the 24-hour quantity.

\section{Ammonia.-}

35. Folin Air Current Method.-The most satisfactory method for determining ammonia in urine is that of Folin. This consists in measuring a given volume of urine into a tall cylinder (B) fitted with a two hole rubber stopper. Through one hole passes a glass tube reaching to the bottom of the cylinder for the admission of compressed air. Through the second hole a glass tube leads into a receiving flask (C) provided with a Folin absorption tube. In this flask is placed a measured volume of $\mathrm{N} / 10$ acid, and enough distilled water to cover the holes in the Folin absorption tube. The ammonia is liberated from the urine by adding solid sodium carbonate.

Into the receiving flask measure 20 c.e. $\mathrm{N} / 10$ acid and add two drops of alizarine red. Add enough distilled water to well cover the bell of the Folin absorption tube. Into the tall cylinder measure 25 e.c. of urine. Cover with a thin layer of kerosene to prevent foaming. Make sure that all connections are tight and everything in readiness to start the air current. The compressed air should be run through $10 \% \quad \mathrm{H}_{2} \mathrm{SO}_{4}$ to remove possible ammonia. Add about $1 \mathrm{gm}$. of anhydrous sodium carbonate to the urine and stopper the cylinder at once. Pass a fairly rapid stream of air through the apparatus, being careful not to blow the contents of the eylinder into the acid bottle. A loose plug of cotton may be introduced in the path of the air passing from urine to acid by including a calcium chloride tube in the circuit. This will prevent the blowing over of any particles of earbonate. Run the air current for $11 / 2$ hours. Disconneet the acid container, rinse and remove the 
Folin absorption tube, and titrate the remaining acid with N/10 alkali.

Subtract the volume of alkali used in titration from the original volume of $\mathrm{N} / 10$ acid. The result is the amount of N/10

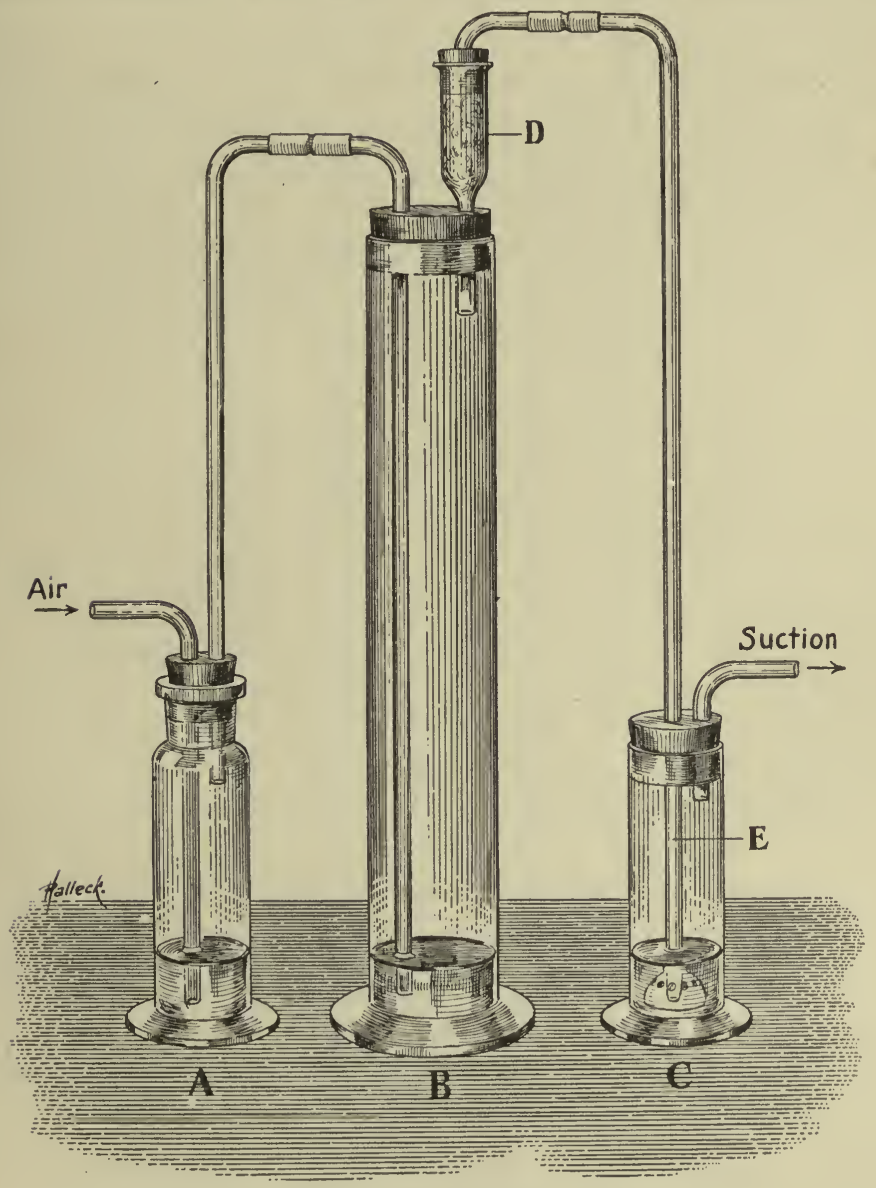

Fig. 4.-Folin's Apparatus for Fistimating Ammonia.

A. Wash bottle containing acid.

B. Tall aerometer cylinder containing urine.

C. Bottle containing standard acid.

D. Calcium chloride tube, loosely packed with cotton wool, to prevent any sodium carbonate being carried over into $\mathrm{C}$.

E. Folin's absorption tube, to bring the air into intimate contact with the acid. 
acid neutralized by the ammonia. One cubic centimeter of N/10 acid corresponds to .0017 grams of $\mathrm{NH}_{3}$ or .0014 grams of $\mathrm{N}$.

Calculate the amount of ammonia nitrogen in the 24 hour specimen and also the per cent of the total nitrogen present as ammonia.

36. Clinical Method. Shiff-Malfatti.-This method depends on the fact that when neutral solutions of ammonium salts are treated with formaldehyde, urotropine (hexamethylene tetramine) is formed and a definite amount of free acid is liberated.

$\left.6 \mathrm{HCHO}+2 \mathrm{NH}_{4}\right)_{2} \mathrm{SO}_{4}=\mathrm{N}_{4}\left(\mathrm{CH}_{2}\right)_{6}+2 \mathrm{H}_{2} \mathrm{SO}_{4}+6 \mathrm{H}_{2} \mathrm{O}$.

This method is not accurate, but serves for most clinical purposes.

Measure 25 c.c. urine into a flask and dilute with 5 volumes of water. Add 4 or 5 drops of phenolphthalein and 5 c.c. of a saturated solution of potassium oxalate. Titrate with N/10 $\mathrm{NaOH}$ to a faint permanent pink.

The formalin solution is prepared by adding 3 volumes of water, a few drops of phenolphthalein, and titrating with $\mathrm{N} / 10 \mathrm{NaOH}$ to a faint pink to neutralize the acid present in the formalin solution. Add 30-40 c.c. of neutralized formalin to the neutralized urine. The color disappears, for free acid is set free as is shown in the above equation. Titrate again to a faint permanent pink. The amount of alkali corresponds to the amount of decinormal ammonia and ammonium salts present in the urine. Calculate the amount of ammonia $\mathrm{N}$ in 25 c.c. of urine, and from this the amount in your 24-hour specimen.

Calculate the per cent of the total nitrogen which is present as ammonia nitrogen.

\section{Urea.-}

37a. Plimmer and Skelton Modification of the Marshall Urease Method.-This method consists in decomposing the urea by means of the enzyme urease, which is found in the soy bean. The nitrogen is converted into ammonia and the ammonia esti- 
mated. Of the various recent modifications of the urease method this is perhaps the best suited for use in a large class unless exceptional laboratory facilities are available.

The apparatus required is identical with that described in the Folin ammonia determination above.

Measure about 50-60 c.c. of distilled water into the tall cylinder. Add $1 \mathrm{gm}$. of finely powdered soy bean (jack bean contains a more active urease), 5 c.c. of urine from a pipette, and kerosene or liquid paraffin to prevent foaming. Connect with a receiving bottle containing 50 c.c. of tenth normal acid accurately measured and two drops of alizarine red. Stand the cylinder in vessels containing water at $35^{\circ}-40^{\circ} \mathrm{C}$. The water in the outside vessel should be kept at this temperature by the addition of hot water as required. Run an air current through the series to drive the liberated ammonia into the acid bottle where it will be absorbed. As the air from the compressed air tap may contain traces of ammonia it should be run through $10 \%$ sulphuric acid washing bottles before being run into the urine cylinder. After about an hour, disconnect the cylinder and add about a gram of anhydrous sodium carbonate to the urine. Connect again and run the air current for another hour. Titrate the excess of acid in the acid bottle with $\mathrm{N} / 10 \mathrm{NaOH}$. Subtracting this value from the total amount of acid used will give the amount of acid neutralized by the liberated ammonia. Calculate the weight of nitrogen liberated from the volume of urine used and from this, the amount present in the total 24-hour specimen. This value represents the amount of nitrogen present as urea + ammonia. Subtract the value found above for ammonia. The result will be the amount of nitrogen present as urea. Calculate the per cent of the total nitrogen which is present as urea.

37b. Colorimetric Method for Determination of Urea in Urine. (Folin: Jour. Biol. Chem., xxvi, 501, 1916; xxxviii, 111, 1919).-

Merck's blood charcoal was a necessary reagent in the deter- 
mination of urea in urine by the direct Nesslerization process of Folin and Denis. By using urease preparations sufficiently free from nitrogenous materials the urea nitrogen can be Nesslerized without any charcoal treatment.

Urease Preparation,-Wash about $3 \mathrm{~g}$. of permutit in a flask once with $2 \%$ acetic acid, then twice with water; add $5 \mathrm{~g}$. of fine Jack bean meal and 100 c.c. of $15 \%$ alcohol (16 c.c. of ordinary alcohol plus 84 c.c. of water). Shake gently but continuously for 10 to 15 minutes, pour on a large filter and cover with a watch glass. The filtrate contains practically the whole of the urease and extremely little of other materials. The urease solution will keep for about a week at room temperatures and for 4 to 6 weeks in an ice box.

Buffer Mixtures for Urease Decompositions.-Mixtures of mono- and disodium-phosphates in the proportion 1 molecule of the former to 2 of the latter, and in molar concentrations, are usually employed to preserve a substantially neutral reaction during the decomposition of urea by means of urease. Dissolve $69 \mathrm{~g}$. of monosodium phosphate and $179 \mathrm{~g}$. of crystallized disodium phosphate in 800 c.c. of warm distilled water. Cool and dilute to a volume of 1 liter.

It would seem to be rather doubtful whether the maintenance of neutrality is adequate to fully explain the accelerating action of phosphates on the urea decomposition, because pyro- and metaphosphates seem to be more effective than orthophosphates. An excellent buffer mixture is obtained by dissolving $14 \mathrm{~g}$. of sodium pyrophosphate $\left(\mathrm{Na}_{4} \mathrm{P}_{2} \mathrm{O}_{7}, 10 \mathrm{H}_{2} \mathrm{O}\right)$ in enough half normal phosphoric acid to make a volume of 100 c.c. The half normal phosphoric acid is made by diluting 20 c.c. of $85 \%$ phosphoric acid to 1 liter and titrating 5 c.c. with tenth normal alkali and phenolphthalein as indicator to a faint pink color. On the basis of this titration dilute the acid to a substantially correct half normal solution. Metaphosphoric acid is fully as good as phosphoric acid, but it is much more difficult to prepare a solution of the requisite degree of neutrality with metaphosphoric acid because of its variable water and free phosphoric acid con- 
tent. The pyrophosphate-phosphoric acid mixture, the preparation of which is described above, gives a faint color with rosalic acid. Five c.c. when titrated with tenth normal alkali and phenolphthalein should give a faint but distinct color with about 18 c.c. of the alkali.

Transfer with an Ostwald pipette 1 c.c. of diluted urea solution or urine (dilution sometimes 5 or 20 , but usually 10 c.c. diluted to 100 c.c.) to a clean test tube; add 1 or at the most 2 drops of buffer mixture and 1 c.c. of urease solution. Digest in a beaker of warm tap water $\left(40^{\circ}\right.$ to $55^{\circ}$ C.) for 5 minutes or at room temperatures for 15 minutes. At the end of the digestion period rinse the contents of the test tube into a 200 c.c. volumetric flask and dilute to a volume of about 150 c.c.

Transfer $1 \mathrm{mg}$. of $\mathrm{N}$ in the form of ammonium sulphate to another 200 c.c. volumetric flask; to this standard add 1 c.c. of urease solution and dilute to about 150 c.c. Then add with shaking (with a eylinder) 20 c.c. of Nessler solution to each. Dilute to volume and make the color comparison, never omitting to first read the standard against itself.

The height of the standard (usually $20 \mathrm{~mm}$.) divided by the height of the unknown, gives the nitrogen, in $\mathrm{mg}$., present in the fraction of a c.c. of undiluted urine present in the 1 c.c. of diluted urine taken for the analysis.

Unless the colorimetric reading is between $14 \mathrm{~mm}$. and $30 \mathrm{~mm}$. the determination should be repeated with 1 c.c. of urine so diluted as to give readings coming within those limits. Calculate the total urea- $\mathrm{N}$ and subtract the preformed ammonia-N.

\section{Uric Acid.-}

Folin-Schaffer Method.-Phosphates and some organic material are first precipitated and filtered off. The uric acid is then precipitated as ammonium urate, and titrated with $\mathrm{N} / 10$ potassium permanganate.

To 200 c.c. of urine in a beaker, add 50 c.c. of Folin-Schaffer reagent ( $50 \%$ ammonium sulphate, $0.5 \%$ uranium acetate and $0.5 \%$ acetic acid). Allow to stand for $1 / 2$ hour and filter through a dry filter. Measure out 125 c.c. of the filtrate (cor- 
responding to 100 c.c. urine), add about 5 c.c. of ammonia and let stand for 48 hours. Filter cautiously through a hard filter, using a glass rod to avoid loss of liquid. Rinse out the beaker several times with $10 \%$ ammonium sulphate solution, each time pouring the rinsing liquid onto the filter after it has drained completely, thus rinsing both beaker and precipitate with the same liquid. This rinsing should be repeated 5 or 6 times.

Carefully remove the filter paper from the funnel, open it and rinse the precipitate into the beaker from which it was just removed, taking care that all the crystals are transferred to the beaker. It is most satisfactory to use hot water for the rinsing. No color should remain on the filter paper. (Note.Do not heat the water in a thick glass wash bottle. Heat in a beaker and pour into the wash bottle). The total volume of the rinsings should be as close as possible to 100 c.c. Cool to room temperature.

Add 15 c.c. of conc. $\mathrm{H}_{2} \mathrm{SO}_{4}$ and titrate the hot liquid at once with $\mathrm{N} / 20$ potassium permanganate. The liquid should be shaken or stirred continuously. At first the color of the permanganate will disappear instantly without diffusing through the liquid. The first instantaneous pink color throughout the entire liquid is taken as the end point of the titration. This color will disappear very quickly, but the addition of another drop also will cause a pink color to diffuse for a very brief time throughout the entire liquid. Each c.c. of $\mathrm{N} / 20$ permanganate $=3.76 \mathrm{mg}$. uric acid. Calculate the amount of uric acid in the volume of urine used (100 c.c.). From this calculate the amount of uric acid in your 24 hour specimen.

From the formula of uric acid calculate the percentage of nitrogen it contains. From this and the results obtained above, calculate the amount of nitrogen in the total uric acid. Calculate the per cent of the total nitrogen present as uric acid nitrogen.

39. Uric Acid. (Folin and Wu: Jour. Biol. Chem., 1919, xxxviii, 81, 459).-

Solutions Required.

1. Standard uric acid solution. Before starting to prepare 
the uric acid solution a $20 \%$ filtered solution of sodium sulfite should be available. Dissolve $1 \mathrm{gm}$. of uric acid in 125 to 150 c.c. of $0.4 \%$ lithium carbonate solution and dilute to a volume of 500 c.c. Transfer 50 c.c. corresponding to $100 \mathrm{mg}$. of uric acid to each of a series of volumetric liter-flasks. Add about 300 e.c. of water and then add 500 c.c. of clear $20 \%$ sodium sulfite solution, mix, dilute to volume, and mix thoroughly. Fill a series of 200 c.c. bottles and stopper very tightly. The reason a series of small bottles is used as containers is, of course, to reduce the absorption of oxygen from the air.

2. A $10 \%$ sodium sulfite solution, kept like the uric acid solution, in small tightly stoppered bottles.

3. A $5 \%$ sodium cyanide solution.

4. A solution containing $5 \%$ of silver lactate and $5 \%$ of lactic acid.

5. The uric acid reagent of Folin and Denis. Boil $100 \mathrm{gm}$. of sodium tungstate with 80 c.c. of phosphoric acid (85\%) and 700 c.c. of water for not less than 2 hours and dilute to 1 liter.

Transfer from 1 to 3 c.c. of urine to a 15 c.e. centrifuge tube and mix with enough water to make a volume of about 6 c.c. Add 5 c.c. of the acid silver lactate solution and stir with a very fine glass rod (diameter 1 to $2 \mathrm{~mm}$.). Rinse off the rod with a few drops of water and centrifuge. If enough silver solution has been added the precipitate settles very quickly. Add a drop of silver lactate solution so as to be sure that an excess is present; if a preciptate is formed add more ( 2 c.c.) of the silver lactate solution and centrifuge again. In point of fact the first 5 c.c. addition of silver lactate is almost always enough, but it is not safe to omit the test. Pour off the clear supernatant liquid as completely as possible.

To the precipitate in the centrifuge tube add, from a burette, 4 c.c. of $5 \%$ sodium cyanide solution and stir until a perfectly clear solution is obtained. Pour the contents into a 100 c.c. volumetric flask and rinse tube and stirring rod, using for this purpose about 15 to 25 c.c. of water. Add 5 c.c. of $10 \%$ sodium 
sulfite solution (to balance the sulfite in the standard uric acid solution). Dilute to a volume of about 50 c.c.

Transfer to another 100 c.c. flask 5 c.c. of the standard uric acid sulfite solution, containing $0.5 \mathrm{mg}$. of uric acid; add 4 c.c. of the cyanide solution and dilute to about 50 c.c. Then add 20 c.c. of saturated sodium carbonate solution to each flask, mix, and finally add with shaking 2 c.c. of the uric acid reagent. Let stand for 3 to 5 minutes, fill to the mark, mix and make the color comparison in the usual manner, never omitting to first read the standard against itself. Artificial light (with "daylite" glass) is better than day light for this color comparison.

With the standard set at $20 \mathrm{~mm}$., 10 divided by the reading of the unknown (in $\mathrm{mm}$.) gives the amount of uric acid (in mg.) in the volume of urine taken.

The discarded blue solution should be poured as directly as possible into the drain pipes of sinks on account of the cyanide.

\section{Hippuric Acid.-}

Hippuric acid may be estimated by the method of Folin and Flanders which consists in hydrolyzing the hippuric acid and estimating the benzoic acid liberated or by that of Kingsbury and Swanson.

\section{Purine Bases.-}

Purine bases may be estimated by a method similar in principle to the Salkowski Method for isolating these compounds. For details consult a larger work.

\section{Creatinine (Folin).-}

The estimation of creatinine is a colorimetric method, based on the Jaffe reaction already familiar to the student. Picric acid is reduced to picramic acid and the red color produced by the latter in alkaline solution is compared with a $N / 2$ potassium bichromate solution in a Duboseq eolorimeter.

Measure 10 c.c. urine into a 500 c.c. volumetric flask ,add 15 c.c. of a saturated solution of picric acid, and 5 c.e. $10 \% \mathrm{NaOH}$. Shake well and allow the mixture to stand 5 minutes. Employ this interval to become familiar with the colorimeter. 
Before putting any liquid into the cups put them in place and run both prisms down until they touch the bottoms of the cylinders. Read both sides of the instrument. Both readings should be 0.0 . If they are not, record the readings carefully and take them into consideration in making readings during the analysis. Be careful about spilling liquid upon the instrument. In case any liquid is spilled upon the instrument, wipe it off carefully.

Pour a little $\mathrm{N} / 2$ potassium bichromate into each of the cylinders of the Duboseq colorimeter. Adjust the depth of the solution in the left hand cylinder exactly to the $8 \mathrm{~mm}$. mark. (If the reading on this side was not 0.0 when the prism was run to the bottom of the cup, make the necessary correction so that the distance between the prism and the bottom of the cylinder is exactly $8.0 \mathrm{~mm}$.) Practice matching the color in the two halves of the field in order to insure greater accuracy in the examination of the unknown. Since the two bichromate solutions are of equal strength, the readings should be identical, no two readings differing by more than 0.1 to $0.2 \mathrm{~mm}$. from the true value $(8 \mathrm{~mm}$.). Always make four readings. Disregard the first, which is apt to be inaccurate, and take the average of the remaining three provided they do not vary more than $0.2 \mathrm{~mm}$.

Exactly at the close of the 5 minutes, dilute the unknown solution to the 500 c.c. mark shaking the flask during the addition of the water to insure complete mixing. Stopper carefully and thoroughly mix the solution. Pour out the bichromate from the right hand cylinder, rinse carefully first with water and then with the solution to be analyzed, and fill the cylinder half full of the liquid. Match the color by moving the prism in the unknown solution, making three or four rapid readings.

Ordinarily 10 c.c. of urine is used for this determination, but if the content of creatinine is above $15 \mathrm{mg}$. or below $5 \mathrm{mg}$. the determination should be repeated with a volume of urine containing an amount of creatinine between 5 and $15 \mathrm{mg}$. This is 
an essential point, as the method is accurate only within these limits.

By experiment it has been determined that $10 \mathrm{mg}$. of pure creatinine gives a color of such strength that $8.1 \mathrm{~mm}$. of such a solution exactly matches $8.0 \mathrm{~mm}$. of bichromate under the above conditions. From the reading obtained with the unknown solution it thus is possible to calculate the amount of creatinine present in the volume of urine used. Remember that the larger the amount of creatinine, the deeper will be the color, and the shorter the column of liquid necessary to match the color of the standard. If a reading of $8.1 \mathrm{~mm}$. corresponds to a creatinine content of $10 \mathrm{mg}$., calculate the amount of creatinine corresponding to the reading of your solution, not forgetting that the proportion will be inverse. Calculate the amount of creatinine in the volume of urine used and in the 24-hour specimen.

From the formula of creatinin calculate what per cent of nitrogen it contains, and then the amount of creatinine nitrogen present in the 24-hour sample. Calculate the per cent of the total nitrogen which this represents.

\section{Microchemical Determination of Creatinin in Urine.} (Folin).-

A suitable and convenient "creatinin reagent" is prepared by adding 75 c.c. of $10 \%$ sodic hydrate to a liter of saturated picric acid solution. If the picric acid is pure and the alkaline solution is kept away from the light and from dust it keeps well for several days. It is usually more safe, however, to prepare only so much of the solution as is used up the same day. For a single determination it is not worth while to prepare the reagent; employ instead the picric acid solution and the alkali, using 20 c.c. of the former and 15 c.c. of the latter.

By means of an Ostwald pipette transfer 1 c.c. or 2 c.c. urine to a 100 volumetric flask. To another similar flask transfer 1 c.e. of a standard creatinin solution ( $1.61 \mathrm{~g}$. of creatinin zinc chlorid dissolved in one liter of tenth normal hydrochloric acid), 1 c.c. of which contains $1 \mathrm{mg}$. of creatinin. To each flask add 20 c.c. of picric acid solution, then add from a buret 1.5 c.c. 
of $10 \%$ sodic hydrate to each, and let stand for ten minutes. If the alkaline solution is used, add, with a cylinder, 20 c.c. to each flask. At the end of ten minutes dilute to the mark with water and mix.

Read the standard against itself in the colorimeter at $20 \mathrm{~mm}$. until the correct value $(20 \mathrm{~mm}$.) can be obtained. The error in reading should not exceed $.2 \mathrm{~mm}$. Rinse the right-hand cup and prism with the unknown, and determine its color in terms of the standard set at $20 \mathrm{~mm}$. Twenty divided by the reading gives the creatinin in milligrams in the quantity of urine taken (1-5 c.c.).

Calculate the total creatinin.

\section{Indican.-}

By applying the qualitative test for indican, already performed by the student, and comparing the color produced with that of Fehling's solution as a standard, at least a comparative, if not a quantitative estimation of indican may be made.

\section{Allantoin.-}

For details of the estimation of allantoin consult a larger work.

\section{Oxalic Acid.-}

Oxalic acid may be estimated by the isolation of its calcium salt.

\section{Chlorides.-}

42. Volhard Method.-All of the chlorides of the urine are precipitated by adding excess (a known volume) of standard silver nitrate. The excess silver nitrate then is determined by titration with thiocyanate, using a ferric salt as indicator. The thiocyanate is made up so that 1 c.c. exactly corresponds to 1 c.c. of the silver nitrate. Silver thiocyanate (white) precipitates first. When all the silver is precipitated, red ferric thiocyanate is formed.

Into a 100 c.c. volumetric flask measure accurately with a 
pipette 10 c.c. urine and 20 c.c. standard silver nitrate solution. Add about 4 c.c. conc. nitric acid and about 5 c.c. iron alum solution. Add distilled water up to the 100 c.c. mark, stopper and mix thoroughly. Filter through a dry filter into a dry vessel. The above process will have precipitated all chlorides as $\mathrm{AgCl}$, and an excess of silver nitrate will be left over.

Accurately measure 50 c.c. of the filtrate into an Erlenmeyer or beaker and titrate the excess of silver with standard thiocyanate to a permanent faint pink or reddish-brown. The amount of thiocyanate used corresponds to the excess of silver remaining in the solution.

One c.c. thiocyanate corresponds to 1 c.c. of the standard silver nitrate.

One c.c. standard silver nitrate corresponds to $0.01 \mathrm{~g} \mathrm{NaCl}$. Since only half the liquid was titrated, multiply the titration figure by two. This gives the excess of silver nitrate remaining.

Subtract the excess of silver solution from the total amount added. This will give the amount of silver solution required to precipitate the chlorides present. Since each cubic centimeter of silver nitrate will precipitate the chloride from 0.01 grams of sodium chloride, the sodium chloride in 10 c.c. of urine may be calculated. From this, calculate the sodium chloride in the 24 hour sample.

\section{Sulphates.-}

The most accurate and satisfactory methods for estimating sulphates are gravimetric. (See Folin's methods.) The sulphates are precipitated by adding barium chloride, the precipitate collected and weighed. Inorganic sulphates are estimated directly. Inorganic + ethereal, after the splitting of ethereal sulphates by boiling with hydrochloric acid. Total sulphur is estimated by fusing the urine with an oxidizing agent and determining the sulphates as above.

Neubauer has suggested the following approximate volumetric method which serves for most clinical purposes: 
Measure 50 c.c. of urine into a flask, add 3 c.c. pure $\mathrm{HCl}$ and boil gently for 15 minutes to decompose the ethereal sulphates. From a burette run in standard barium chloride solution ( 1 c.c. $=0.01 \mathrm{gm}$. $\mathrm{SO}_{3}$ ) as long as a precipitate forms, the mixture being kept hot. After running in the first 3-4 c.c. of barium chloride, allow the precipitate to settle and with a glass rod remove a drop of the liquid. Place it on a watch glass over a black surface and add a few drops of the $\mathrm{BaCl}_{2}$ solution. If there is a precipitate, return the whole to the flask, rinsing in the last traces with water, and add more $\mathrm{BaCl}_{2}$. Again allow to settle and test as before. Proceed until no more $\mathrm{BaSO}_{4}$ is precipitated. Excess of $\mathrm{BaCl}_{2}$ must be avoided. When the minimal excess has been added, a drop of the clear fluid removed from the flask will give only a cloudiness with a drop of dilute $\mathrm{H}_{2} \mathrm{SO}_{4}$. If more than a cloudiness appears, excess has been added and the whole operation must be repeated. Calculate the amount of $\mathrm{SO}_{3}$ present in 50 c.c. urine and in the 24 hour sample.

\section{Phosphates.-}

When a solution of disodium phosphate acidified with acetic acid is treated with a solution of uranium acetate, a white precipitate of uranium phosphate is formed. To determine the end point, a drop of the liquid is brought in contact with a drop of potassium ferrocyanide on a white tile. Any excess of uranium causes a brown color to appear.

To 50 c.c. of urine in a small beaker, add 5 c.c. of a special sodium acetate mixture (100 g. sodium acetate dissolved in 800 c.c. water plus 100 c.c. $30 \%$ acetic acid and the volume made up to 1 liter). This changes any acid phosphate into diacid sodium phosphate. Heat to boiling and from a burette run in drop by drop a standard uranium acetate mixture ( 1 c.c. is equivalent to $0.005 \mathrm{~g}$. of $\mathrm{P}_{2} \mathrm{O}_{5}$ ). Keep the mixture at the boiling point. From time to time remove a drop with a glass rod or dropper and bring it in contact with a drop of potassium ferrocyanide on a white tile. If a glass rod is used it should be rinsed before replacing it in the beaker. It 
is convenient to arrange a tile with several drops of ferrocyanide before beginning the titration. The first appearance of brown is taken as the end point. Calculate the weight of $\mathrm{P}_{2} \mathrm{O}_{5}$ represented by the phosphates in 50 c.c. of urine, and from this the amount in the 24 hour sample. Calculate what weight of phosphorus this represents.

\section{Pathologic Urine}

Some substances occur in pathological urine which are absent from, or found only in traces in normal urine. Important among these substances are various proteins, carbohydrates, and the acetone bodies.

Proteins.-The proteins most frequently found are albumin, globulin, nucleo-protein, hemoglobin, glycoprotein; of the protein derivatives, metaproteins, proteoses, peptones and amino acids.

As turbidity would interfere with the detection of small amounts of protein, the urine should be clear before testing. If necessary, filter, repeating the filtration and adding a small amount of bismuth subnitrate if the filtrate did not come through clear. If the urine still remains cloudy, add a drop or two of barium chloride and then a drop or two of sodium carbonate solution. Barium carbonate precipitates and carries down with it the suspended material.

\section{Albumins and Globutins.}

45. Heat T'est.-The heat test should be made in acid solution, as only in a weak acid solution will the proteins coagulate properly on boiling. The urine should be tested with litmus and acidified if necessary with $0.5 \%$ acetic acid. Even if the urine is acid, it is well to add a few drops of dilute $(0.5 \%)$ acetic acid to insure coagulation of proteins possibly present.

Boil a few cubic centimeters of acidified urine. If no precipitate forms, albumin and globulin are absent. If it becomes cloudy or a precipitate forms, albumin or globulin is present. 
Do not add acetic acid before heating: (1) because albumin, if present, may be converted into acid albumin; (2) because acid tends to disintegrate cellular elements which may be present, with the consequent separation of cell-proteins. The use of strong mineral acids in this test is objectionable for similar reasons.

46. Heller's Nitric Acid Test.-To four c.c. of urine in a test tube add, by means of a pipette, a few cubic centimeters of concentrated nitric acid. Run in carefully, so that two distinct layers may be formed. In the presence of albumin or globulin, a distinct white ring will form at the junction of the two layers.

The following important facts should be taken into consideration: Not over five minutes should be occupied in this test, since prolonged action of nitric acid will separate cellproteins, which may be present.

When urea is present in large quantities, particularly if the volume of the urine is low, crystals of urea nitrate may be deposited at the line of contact of the two liquids. The crystalline character of this material suffices for differentiation.

In the presence of large quantities of uric acid, a whitish ring may appear several $\mathrm{mm}$. above the line of contact. If doubt exists as to either of these two conditions, dilute the urine and repeat the test. If the ring was due to uric acid or urea, it will fail to appear after dilution. A cloud, high up in the urine, may be caused by mucin. The pigments form rings of dark color between the urine and the acid. These rings, unce recognized, eannot be mistaken for proteins. A ring due to certain ingested substances, e. g., copaiba, may be dissolved with ether; iodine, excreted in the urine, forms a dark ring at the surface of the nitric acid. Indican is the most common pigment productive of colored rings.

47. Ferrocyanide Test.-Add a few drops of potassium ferrocyanide solution to the clear urine and then acidify with acetic acid. In the presence of albumin or globulin, a white cloud or 
ring will be formed. Observed in a strong light, this will be seen to be of a flocculent character.

48. Quantitative Estimation, (Approximate.)-Pour the urine into an Esbach tube up to the mark "urine" or U. Add Esbach's reagent ( $2 \%$ citric acid, $1 \%$ picric acid) to the mark "reagent" or $R$, close the tube carefully with a stopper and invert several times until the fluids are well mixed. Allow the tube to stand 24 hours. Read on the scale the amount of the precipitate. The scale corresponds to grams protein per 1000 e.c. of urine.

Although this method gives only approximate results it usually is sufficient for clinical purposes.

Serum Globulin Differentiation.

49. Make a few cubic centimeters of clear urine alkaline with ammonia. Filter off the precipitated phosphates. Neutralize the filtrate with acetic acid and treat with an equal volume of saturated ammonium sulphate solution. Globulin, if present, will be thrown down. If the urine is rich in urates they may be precipitated but may be recognized with the microscope or by the murexid test.

50. To a few cubic centimeters of clear urine, add an excess of solid magnesium sulphate. Shake thoroughly and globulins, if present, will be precipitated and will be deposited above the excess of salt.

Add a few drops of clear urine to a large beaker full of distilled water. If globulins are present, a white cloud will appear.

\section{Nucleoprotein.}

After ascertaining that the clear urine does not coagulate upon heating, dilute a small amount with water in equal quantities, to prevent the precipitation of uric acid and to reduce the solvent action of the salts upon the nucleoprotein. Add acetic acid drop by drop. Nucleoprotein, if present, will be precipitated.

Nucleoproteins often are present in large quantities in various forms of proteinuria, such as physiologic proteinuria 
(occurring at times in healthy individuals after excessive exertion, etc.), orthostatic proteinuria (a form in which the protein disappears from the urine if the patient is kept lying down), and lordotic proteinuria (a form accompanying lordosis, a variety of spinal curvature).

It should be borne in mind, however, that the nucleoprotein may have its origin from disintegrated cells, which may be present in large amount.

\section{Metaprotein.}

The proteins present in the urine, may be converted, by acids or alkali present, into the metaproteins. Ascertain the chemical reaction of urine and neutralize clear urine carefully. If precipitation occurs, heat; the derived albumin will be coagulated and will not redissolve on adding acid or alkali.

Proteoses and Peptones.

These forms may appear in the urine occasionally.

52. Neutralize and boil the clear urine to remove coagulable proteins. Filter and to the filtrate, slightly warm, add a few drops of potassium ferrocyanide solution and $10 \%$ acetic acid. If a precipitate appears, it is proteose. Heat and it will disappear; cool and it will reappear.

53. To clear urine, previously acidified and boiled, add excess of solid ammonium sulphate and a few drops of acetic acid; proteoses, if present, will be precipitated. Filter. Dissolve the precipitate in distilled water and apply the biuret test. Ammonium sulphate interferes with the reaction and must be removed with barium carbonate.

A specimen of urine may be dialyzed and the diffusate tested for peptones with the biuret test.

\section{Carbohydrates.-}

The carbohydrates most firequently found in the urine are dextrose and lactose. In addition to these, pentoses, levulose, galactose, and saccharose may occur. These substances are detected by the usual reduction, phenylhydrazine and fermentation tests, or by the optical activity of the urine containing 
them. For differentiating among the various carbohydrates, the methods employed are those already familiar to the student from his study of the carbohydrates.

With samples of the carbohydrate urine furnished perform the following tests:

Reduction Tests.-Albumin or globulin must be removed if 'present by acidifying slightly with acetic acid, boiling and filtering.

54. Qualitative Test.-Perform the Fehling test. The test is subject to two sources of error: unless the liquid is boiled the test is not sensitive; if boiled, other substances such as uric acid, creatinine, mucin, pentoses, glycuronic acids, lactose, or a reducing agent used in the preservation of the urine, such as chloroform or formaldehyde may give a reduction.

55. Perform the Benedict test.

Quantitative Estimation of Sugar.-The quantitative estimation of sugar in urine is attended by some difficulty, and a variety of methods have been proposed. Only two titration methods will be included here, first the Fehling method, because it is the standard method upon which most later and more accurate methods are based, and second Benedict's method, which is perhaps the best for general use, all things considered.

56. Fehling Method.-The Fehling test may be made quantitative by making up the copper sulphate solution accurately. A given amount of dextrose will reduce a definite amount of copper. As long as copper sulphate remains unreduced the solution will have a blue color. A measured volume of Fehling's solution may thus be titrated with the dextrose urine until the color has completely disappeared. From the volume of urine necessary to reduce the given volume of Fehling's solution, the amount of dextrose in the urine may be calculated.

The quantitative Fehling solution is made up of such a strength that 10 c.c. of the copper solution (20 c.c. of the mixed Fehling's) will be reduced by $0.1 \mathrm{gm}$. glucose or 0.134 gm. lactose. 
Before starting the experiment read through the entire directions for the process.

With a pipette measure 10 c.c. of each part of the quantitative Fehling's solution into an Erlenmeyer flask (or evaporating dish). Add 80 c.c. of water measured with a graduate. Dilute 5 c.c. of urine (measured with a pipette) with 20 c.c. distilled water (also measured with a pipette). Diluting in a volumetric flask is preferable, but the method described is satisfactory.

Fill a burette with the diluted urine. Heat the diluted Fehling's solution in the Erlenmeyer with a small flame, and run in the urine, first a few drops at a time, boiling a few seconds after each addition. If the liquid remains blue after the addition of 2 c.c. of urine, continue the process, adding the urine in $0.2-0.3$ c.c. portions until the blue or green color has almost disappeared, then drop by drop until no blue or green tinge can be detected. Observe over a white paper. Do not look through the liquid at the blue sky. The volume of urine required to reduce the 20 c.c. of Fehling's will contain $0.1 \mathrm{gm}$. dextrose or $0.134 \mathrm{gm}$. lactose. Perform duplicate analyses. Time may be saved by running through a trial titration before the actual determination, in order to find roughly the volume of urine required to reduce the Fehling's solution.

Calculate the weight of sugar in 100 c.c. of urine. If the sugar content of the urine is so high that less than 2 c.c. are required to reduce 20 c.c. of Fehling's solution, the urine must be further diluted.

57. Benedict's Method.-The greater accuracy of this method is due to several facts: the solution is less strongly alkaline, so that the decomposition of the sugar on boiling is less, and the end point is perhaps sharper than in the original Fehling method.

Standard Copper Solution.-Eighteen grams pure erystalline $\mathrm{CuSO}_{4} ; 200$ grams crystalline $\mathrm{Na}_{2} \mathrm{CO}_{3} ; 200$ grams sodium or potassium citrate; 125 grams KCNS; 5 c.c. of a $5 \%$ solution of $\mathrm{K}_{4} \mathrm{Fe}(\mathrm{CN})_{6}$; distilled water to make a total volume of 1 liter. 
Dissolve the carbonate, citrate and thiocyanate in about 700 c.c. of water and filter if necessary. Dissolve the copper sulphate in 100 c.c. of water, and add slowly, stirring constantly, to the 700 e.c. Add the ferrocyanide and make up to exactly 1 liter. The only ingredient which need be weighed exactly is the copper sulphate.

Analysis.-Measure 25 c.c. (pipette) of the reagent into a 25-30 em. evaporating dish. Add 10-20 grams crystalline $\mathrm{Na}_{2} \mathrm{CO}_{3}$, or $1 / 2$ this amount of the anhydrous salt, some talcum or powdered pumice and heat to boiling over a free flame until the carbonate has dissolved.

Accurately dilute 10 c.c. of urine to 100 c.c. unless the amount of sugar in it is small, when it can be used without dilution. Fill a burette with the urine, and run it into the boiling copper solution, rapidly at first and then more slowly as the color grows less, then a few drops at a time until the solution is colorless. A white precipitate forms during the titration. If the liquid in the dish becomes too concentrated, add water.

Calculation.-Exactly $50 \mathrm{mg}$. of glucose will reduce the 25 c.c. of the copper reagent. This amount of glucose must have been present in the volume of urine used, provided no other reducing substances were present. If the urine was diluted 10 times, then the per cent of glucose in the original urine was $\frac{0.050 \times 1000}{\mathbf{x}}$ where $\mathrm{x}$ is the volume of urine used from the burette.

58. Fermentation Tests.

Perform a fermentation test on carbohydrate urine.

This test is a very satisfactory one, as the other reducing substances in the urine do not ferment. It furnishes also a means of differentiation between dextrose and lactose as certain varieties of yeast will ferment dextrose but not lactose. 
Phenylhydrazine Test.

This test is carried out as described under carbohydrates, and may be used to distinguish various members of the group.

Optical Activity.

In testing for sugars with the polariscope it must be remembered that other substances, such as proteins, ete., may be the cause of any observed rotation.

\section{Acetone Bodies.-}

The term "acetone bodies" includes acetone, $\beta$-oxybutyric acid and acetoacetic, and this is readily converted into acetone by the loss of $\mathrm{CO}_{2}$, so that these acids are never found in the urine unaccompanied by acetone. Acetone may oceur, however, when these acids are not present.

Acetone, if present in large amounts, may be tested for in the urine directly. If only small amounts are present, the urine may be distilled. The acetone will go over in the first few cubic centimeters of distillate. An acetone urine will be supplied containing sufficient acetone to give the tests.

59. Rothera's T'est.- Saturate about 10 c.c. of urine with ammonium sulphate. Add 2-3 drops of fresh sodium nitroprusside solution and 2-3 c.c. concentrated ammonia. Mix and allow to stand at least $1 / 2$ hour. A characteristic permanganate color indicates the presence of acetone.

60. Iodoform T'est.-Perform the iodoform test with acetone urine. (See under Fermentation.) 


\section{APPENDIX}

\section{DIRECTIONS FOR MAKING UP QUANTITATIVE OR SPECIAL REAGENTS}

\section{Ammonium Thiocyanate, Standard, for Chlorides.-}

One c.c. is equivalent to 1 c.c. standard $\mathrm{AgNO}_{3}$.

This solution is made of such a strength that 1 c.c. of it is equal to 1 c.c. of the standard silver nitrate solution mentioned below. To prepare the solution dissolve 12.9 grams of ammonium thiocyanate, $\mathrm{NH}_{4} \mathrm{SCN}$, in a little less than a liter of water. In a small flask place 20 c.c. of the standard silver nitrate solution, 5 c.c. of a cold saturated solution of ferric alum and 4 c.c. of nitric acid (sp. gr. 1.2), add water to make the total volume 100 c.c., and thoroughly mix the contents of the flask. Now run in the ammonium thiocyanate solution from a burette until a permanent red-brown tinge is produced. This is the end-reaction and indicates that the last trace of silver nitrate has been precipitated. Take the burette reading and calculate the amount of water necessary to use in diluting the ammonium thiocyanate in order that 10 c.c. of this solution may be exactly equal to 10 c.c. of the silver nitrate solution. Make the dilution and titrate again to be certain that the solution is of the proper strength.

\section{Barfoed's Solution.-}

Dissolve 4.5 grams of neutral, crystallized copper acetate in 100 c.c. of water and add 1.2 c.c. of $50 \%$ acetic acid.

\section{Barium Chloride for Sulphate Determination.-}

Thirty and five-tenths grams $\mathrm{BaCl}_{2} \cdot 2 \mathrm{H}_{2} \mathrm{O}$ made up to 1 liter with distilled water.

One c.c. corresponds to 0.01 grams $\mathrm{SO}_{3}$. 


\section{Benedict's Solution for Carbohydrate Estimation.-}

See under description of the method in the laboratory directions.

\section{Congo Paper.-}

Dissolve 1 gram of Congo red in 90 c.c. water, add 10 c.c. $95 \%$ alcohol. Dip filter paper into this solution, and allow to dry.

\section{Esbach's Reagent.-}

Dissolve 10 grams of picric acid and 20 grams of citric acid in 1 liter of water.

\section{Fehling's Solution. (Quantitative.)-}

Fehling's solution is composed of two definite solutionsa copper sulphate solution and an alkaline tartrate solution, which may be prepared as follows:

$A$. Copper sulphate solution $=34.65$ grams of copper sulphate dissolved in water and made up to 500 c.c.

$B$. Alkaline tartrate solution $=125$ grams of potassium hydroxide and 173 grams of Rochelle salt dissolved in water and made up to 500 c.c.

These solutions should be preserved separately in rubberstoppered bottles and mixed in equal volumes when needed for use. This is done to prevent deterioration.

Only the copper salt need be weighed on the quantitative balance.

\section{Fehling's Solution. (Qualitative.)-}

Amounts as in quantitative, but weighed on the ordinary balance.

\section{Folin-Schaffer Reagent.-}

This reagent consists of 500 grams of ammonium sulphate, 5 grams of uranium acetate, and 60 c.c. of $10 \%$ acetic acid in 650 c.c. of distilled water. Make up to 1 liter.

Folin's reagents for uric acid, urea, creatinine, ete. See the individual methods. 


\section{Formalin. (Neutral.)-}

Dilute formalin 1-4 with distilled water, add a small amount of phenolphthalein, and then add dilute (N/10) sodium hydrate until the liquid acquires a faint pink tinge.

\section{Glyoxylic Acid Solution.-}

Hopkins-Cole Reagent (Benedict's Modification).-Ten grams of powdered magnesium are placed in a large Erlenmeyer flask and shaken up with enough distilled water to liberally cover the magnesium. Two hundred and fifty cubic centimeters of a cold, saturated solution of oxalic acid is now added slowly. The reaction proceeds very rapidly and with the liberation of much heat, so that the flask should be cooled under running water during the addition of the acid. The contents of the flask are shaken after the addition of the last portion of the acid and then poured upon a filter, to remove the insoluble magnesium oxalate. A little wash water is poured through the filter, the filtrate acidified with acetic acid to prevent the partial precipitation of the magnesium on long standing, and made up to a liter with distilled water. This solution contains only the magnesium salt of glyoxylic acid.

\section{Guenzberg's Reagent.-}

Dissolve 2 grams of phlorglucinol and 1 gram of vanillin in 100 c.c. of $95 \%$ alcohol.

\section{Magnesia Mixture.-}

Dissolve 175 grams of magnesium sulphate and 350 grams of ammonium chloride in 1,400 c.c. of distilled water. Add 700 grams of concentrated ammonium hydroxide, mix thoroughly, and preserve the mixture in a glass-stoppered bottle.

\section{Mett's Tubes.-}

See directions for making in chapter on gastric digestion.

\section{Millon's Reagent.-}

Digest 1 part (by weight) of mercury with 2 parts (by 
weight) of nitric acid (sp. gr. 1.42) and dilute the resulting solution with 2 volumes of water.

Molisch's Reagent.-

A $15 \%$ alcoholic solution of $\propto$ naphthol.

Nylander's Reagent.-

Digest 2 grams of bismuth subnitrate and 4 grams of Rochelle salt in 100 c.c. of a $10 \%$ solution of potassium hydroxide. The reagent should then be cooled and filtered.

Pancreatic Solution. ("Artificial pancreatic juice.")-

One per cent neutral solution of pancreatic powder in water.

Pepsin Solution. (Artificial gastric juice.)-

One per cent solution of pepsin flakes in $0.2 \% \mathrm{HCl}$.

Potassium Bichromate N/2.-

Dissolve 24.55 grams pure potassium bichromate in distilled water and make up to 1 liter of solution.

\section{Potassium Permanganate $\mathbf{N} / 20$.}

Dissolve 1.578 grams of the pure salt in distilled water and make up to 1 liter.

\section{Potassium Pyroantimonate $\mathrm{K}_{2} \mathrm{H}_{2} \mathrm{Sb}_{2} \mathrm{O}_{7}$. -}

Two grams of $\mathrm{K}_{2} \mathrm{H}_{2} \mathrm{Sb}_{2} \mathrm{O}_{7}$ are added to 100 c.c. of boiling water, the mixture is boiled until the salt is dissolved, and the solution quickly cooled. Three c.c. of $10 \% \mathrm{KOH}$ solution is added to it to render the reagent alkaline. (Bray.)

Silver Nitrate (Standard) for Volhard Chloride Method.-

One c.c. is equivalent to 0.01 grams $\mathrm{NaCl}$.

Dissolve 29.042 grams of pure silver nitrate in distilled water and make up to 1 liter.

\section{Sodium Cobaltinitrite $\mathrm{Na}_{3} \mathrm{Co}\left(\mathrm{NO}_{2}\right)_{6}$.-}

One hundred grams of $\mathrm{NaNO}_{2}$ are dissolved in 200 c.c. of water, and to this solution 50 c.c. of 6 -molar acetic acid and 
10 grams of $\mathrm{Co}\left(\mathrm{NO}_{3}\right)_{2} .6$ aq. are added. After a day or two the solution is filtered from any precipitate, $\mathrm{K}_{2} \mathrm{Na}\left[\mathrm{Co}\left(\mathrm{NO}_{2}\right)_{6}\right]$. aq. and diluted to 400 c.c. (Bray.)

Special Sodium Acetate Solution (for Uranium Acetate Method for Phosphates.) -

Dissolve 100 grams of sodium acetate in 800 c.c. of distilled water, add 100 c.c. of $30 \%$ acetic acid to the solution, and make the volume of the mixture up to 1 liter with distilled water.

\section{Stokes' Reagent.-}

A solution containing $2 \%$ ferrous sulphate and $3 \%$ tartaric acid. When needed for use a small amount should be placed in a test tube and ammonium hydroxide added until the precipitate which forms on the first addition of the hydroxide has entirely dissolved. This produces ammonium ferrotartrate, which is a reducing agent.

\section{Toepfer's Reagent.-.}

Dissolve 0.5 grams of dimethylamino-azobenzene in 100 c.c. of $95 \%$ alcohol.

\section{Uranium Acetate Solution (Standard).-}

Dissolve about 35.0 grams of uranium acetate in 1 liter of water with the aid of heat and $3-4$ c.c. of glacial acetic acid. Let stand a few days and filter. Standardize against a phosphate solution containing 0.005 gram of $\mathrm{P}_{2} \mathrm{O}_{5}$ per cubic centimeter. For this purpose dissolve 14.721 grams of pure air-dry sodium ammonium phosphate $\left(\mathrm{NaNH}_{4} \mathrm{HPO}_{4} \cdot 4 \mathrm{H}_{2} \mathrm{O}\right)$ in water to make a liter. To 20 c.c. of this phosphate solution in a 200 c.c. beaker add 30 c.c. of water and 5 c.c. of sodium acetate solution (see above) and titrate with the uranium solution to the correct end reaction as indicated in the method proper. If exactly 20 c.c. of uranium solution are required, 1 c.c. of the solution is equivalent to 0.005 gram $\mathrm{P}_{2} \mathrm{O}_{5}$. If stronger than this, dilute accordingly and check again by titration. 


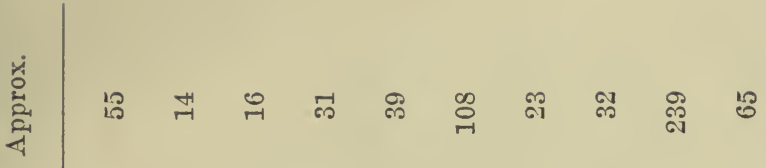

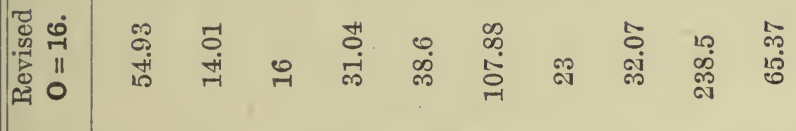

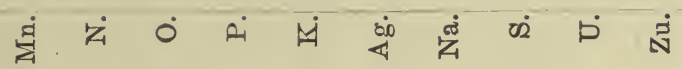

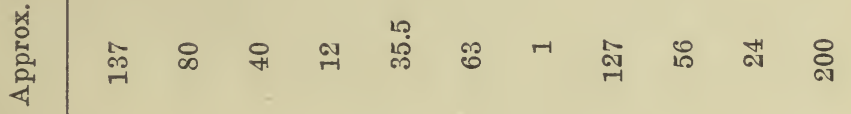

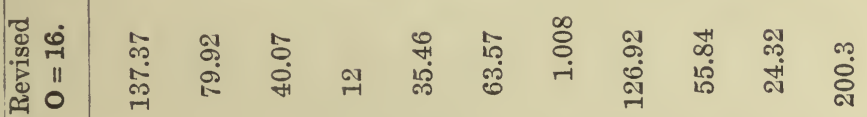

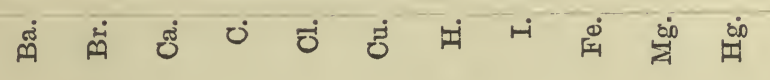

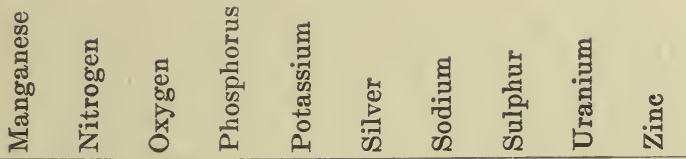

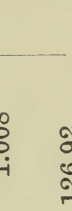





\section{REFERENCES}

The following list will furnish the student with a nucleus of reference material. Current journals such as the Journal of Biological Chemistry, the Biochemical Journal, and Chemical Abstracts should be consulted. The ten-year index of the last named journal has now been issued, and serves as an invaluable survey of biochemical work from 1907 to 1916 .

\section{General Texts or Reference Works}

Hammarsten-Mandel: T'extbook of Physiological Chemistry, John Wiley \& Sons, New York, 1914.

MatThews: Physiological Chemistry, William Wood \& Co., New York, 1921. ABDERHALden: Lehrbuch der Physiologischen Chemie, 1914, 1915.

VoN Fürth: Probleme der Phys. u. Path. Chemie, Vogel, Leipzig, 1912. Abderhalden: Biochemisches Handlexicon, Springer, Berlin. A large work of reference in several volumes.

CzapeK: Biochemie der Pflanzen, G. Fischer, Berlin. 1913.

BArger: The Simpler Natural Bases (Plimmer Monograph), Longmans, Green \& Co., New York, 1914.

\section{For Laboratory Methods}

ABderhalden: Handbuch der Biochemischen Arbeitsmethoden, Urban and Schwarzenburg. A large work in many volumes.

HAwk: Practical Physiological Chemistry, P. Blakiston's Son \& Co., Philadelphia, 1921.

Plimmer: Practical Organic and Biochemistry, Longmans, Green \& Co., New York.

Frndlay: Osmotic Pressure, Longmans, Green \& Co., New York, 1913.

\section{Fats}

Leathes: The Fats, Longmans, Green \& Co., New York, 1910.

\section{Carbohydrates}

Armstrong: Simple Sugars and Glucosides, Longmans, Green \& Co., New York.

NEF, J. U.: Dissoziationsvorgänge in der Zuckergruppe, Annalen der Chemie, 1913, 403, 204.

\section{Enzymes}

HARden: Alcoholic Fermentation (Plimmer Monograph), Longmans, Green \& Co., New York, 1911.

EULER: The General Chemistry of Enzymes, Trans. by Pope, 1912.

CoHnherm: Enzymes, John Wiley \& Sons, New York, 1912. 
OPPENHeImer: Die Fermente u. ihre Wirkungen, Vogel, Leipzig, 1913.

BAyliss: Nature of Enzyme Action (Plimmer Monograph), Longmans, Green \& Co., New York, 1914.

\section{Proteins}

Schryver: General Character of Proteins (Plimmer Monograph), Longmans, Green \& Co., New York, 1909.

ManN: Chemistry of the Proteids, Macmillan Co., New York.

Osborne: The Vegetable Proteins (Plimmer Monograph), Longmans, Green \& Co., New York, 1912.

Plimmer: Chemical Constitution of the Proteins, Longmans, Green \& Co., New York, Part I, 1912; Part II, 1913.

Underhill: Phýsiology of the Amino Acids, Yale University Press.

Kossel: Protamines and Histones, Longmans, Green \& Co., New York, 1914.

Jones: Nucleic Acids (Plimmer Monograph), Longmans, Green \& Co., New York, 1914.

See also a list of 54 references in MATTHEws, pages 187-189, 1921 edition.

\section{Colloids}

Ostwald: Grundriss der Colloid Chemie, 1909.

TAylor: Chemistry of Colloids, Longmans, Green \& Co., New York, 1915. HatscheK: Introduction to the Physies and Chemistry of Colloids, P. Blakiston's Son \& Co., Philadelphia, 1916.

Holmes: Laboratory Manual, John Wiley \& Sons, 1922.

\section{Fcods}

Composition of Foods, U. S. Dept. of Agriculture, O. E. S. Bull. No. 221, 1909.

LEACH: Food Inspection and Analysis, Joln Wiley \& Sons, New York.

Mendel: Changes in the Food Supply, Yale University Press, 1916.

\section{Brain}

Levene And JACoBs: Cerebrosides of Brain, Journal Biological Chemistry, 1912, xii, 381 and 389 .

\section{Blood}

VAN SLyke AND MEYer: Amino Acid Nitrogen of the Blood, Journal Biological Chemistry, 1912, xii, 399.

ABDERHALDEN: Amino Acids in Blood, Zeitschrift für physiologische Chemie, 1913, lxxxviii, 480.

ABDerhalden: Abwehrfermente, 1914.

VoN Hess AND MCGUIGAN: Sugar Content Determined by Vivi-diffusion, Journal Pharmacology and Experimental Therapeuties, 1914, vi.

ABEL: Vivi-diffusion, Journal Pharmacology and Experimental Therapeutics, 1914, iv, 611. 
Gradwohl-Blaivas: The Newer Methods of Blood and Urine Chemistry, C. V. Mosby Co., St Louis, 1921.

MYers: Practical Chemical Analysis of Blood, C. V. Mosby Co., St. Louis, 1921.

\section{Digestion}

BeAumont: Physiology of Digestion, 1847.

Pavlov: The Work of the Digestive Glands, Trans. by Thompson, 1910.

BABKIN: Die Aüssere Sekretion der Verdauungsdrüsen, Springer, Berlin, 1914.

Bernafd: Memoire sur le pancreas, Paris, 1856.

Bayliss and Starling: Enterokinase, Journal of Physiology, 1903, xxx, 61.

BAyliss AND Starling: Secretin, Journal of Physiology, 1902, 29 and 31.

Carlson, etT AL.: Journal American Medical Association, 1916, lxvi, 178.

Wohlgemuth: Composition of Pancreatic Juice, Biochemische Zeitschrift, 1912, xxxix, 321.

Mellanby AND Woolley: Trypsin and Trypsinogen, Journal of Physiology, 1914, xlvii, 339.

Mellanby and Woolley: Ferments of the Pancreas, Journal of Physiology, 1915, xlix, 246.

Hammarsten: Bile, Ergebnisse der Physiologie, 1905.

Pregl: Cholic Acid, Zeitschrift für physiologische Chemie, 1910, lxv, 157. LIFSOrÜTz: Cholic Acid, Berichte der deutschen chemischen Gesellschaft, 1914, xlvii, 1459.

Fischer AND Rose: Composition of Bilirubin, Zeitschrift für physiologische Chemie, 1914, lxxxix, 262.

Grindley and MaCNEal: Bacteria and Chemistry of Feces of Healthy Men, Studies in Nutrition, Vols. 3, 4, and 5, University of Illinois, 1912.

\section{Urine}

Neuberg: Der Harn, Springer, Berlin, 1911.

FourN: Laws Governing the Chemical Composition of the Urine, American Journal Physiology, 1905, xiii, 66, 45; Also numerous other papers by Folin in the Zeitschrift für Physiologische Chemie, Journal of Biological Chemistry, American Journal of Physiology, and elsewhere.

A System of Blood Analysis, Journal Biological Chemistry, xxxviii, 81, 1919.

Gradwohl-Blatvas: The Newer Methods of Blood and Urine Chemistry, C. V. Mosby Co., St. Louis, 1921. A short volume giving some of the more recent analytical methods. 


\section{Metabolism}

The literature of metabolism is enormous. The following brief list will serve as the handle of the fan, from which the usually abundant references will lead out into the broader or more specialized aspects of the field.

\section{Energy Exchange}

LAvoisier: Publications on Animal Heat, Histoire et Memoires de l'Acad. de Sci., Paris, 1777-1789.

RUBNER: Zeitschrift für Biologie, 1894, xxx, 73.

Atwater and Benedict: Metabolism of Matter and Energy in the Human Body, U. S. Dept. Agriculture O. E. S. Bull., No. 136, 1903.

Russell Sage Institute of Pathology: Clinical Calorimetry, 1910-22. (Papers by Lusk, du Bois, Benedict and others.)

\section{General}

Von Noorden: Metabolism and Practical Medicine, Keener, Chicago, 1907. LUsk: The Science of Nutrition, W. B. Saunders Co., 1917.

VoN Fürth: Probleme der Physiologischen u. Pathologischen Chemie, 1912. CATHCART: The Physiology of Protein Metabolism (Plimmer Monograph),

Longmans, Green \& Co., New York, 1912.

TAYLOR: Digestion and Metabolism, Lea \& Febiger, Philadelphia, 1912. DAKIN : Oxidations and Reductions in the Animal Body (Plimmer Monograph), Longmans, Green \& Co., New York, 1912.

ALLEN: Glycosuria and Diabetes, 1913.

Paton: Nervous and Chemical Regulator of Metabolism, Macmillan Co.,

New York, 1913.

McCAY: The Protein Element in Nutrition, Longmans, Green \& Co., New York, 1912.

Fletcher: The A B C of Our Own Nutrition.

Albu-Neuberg: Mineral Stoffwechsel, Springer, Berlin, 1906.

VINCENT: Internal Secretion and the Ductless Glands, Arnold, London, 1913.

BENEDICT: The Influence of Inanition on Metabolism, Carnegie Institute Report, Washington, 1907.

Benedict: Experiment on a Man Fasting Thirty-one Days, Carnegie Institute, Pub. 203, 1915.

Lusk: Fundamental Basis of Nutrition, Yale University Press, 1915.

Lectures on Nutrition, Journal of the Washington Academy of Science, 1916, vi.

\section{Vitamines}

FUnK: Lancet, London; Journal of Physiology; and Biochemical Journal from 1911 on. 
Numerous papers by Osborne, Mendel, McCollum and others, mostly in the Journal of Biological Chemistry, 1912 on.

Funk: Review, References to literature, American Medicine, 1916, xi, 751. Voegthin: Review, Journal Washington Academy of Science, 1916, vi, 575; Scientific Monthly, 1916, ii, 289.

Chamberlarn: Prevention of Beriberi among Philippine Scouts, Journal American Medical Association, 1915, lxiv, 1215.

Funk: The Vitamines, Williams \& Wilkins, 1922.

EDDY: Vitamin Manual, Williams \& Wilkins, 1921.

Chemical Abstracts appearing twice monthly since 1907 is an invaluable aid in referring to the enormcus literature on biochemical material. 



\section{INDEX}

(See also separate index for laboratory work)

A

Abderhalden, reaction, 142

Absorption, 173-175

general, 173

in intestine, 173

in mouth, 173

of carbohydrates, 174

of fats, 175

of proteins, 174

spectra, 68

Acetone bodies, in diabetes, 213

Acidity of gastrie contents, 156 in disease, 157

Acrolein test, 84

Adamkiewicz test, 102

Adenine, 186

Adrenalin, 205, 224

Adsorption, 33

Albuminates, (sfe Metaproteins)

Albuminoids, 92, 112

Albumins, 92, 111

Alcaptonuria, 178

Alcohol, as protein precipitant, 105 utilization of, 220

Alkaloidal reagents, as protein precipitants, 105

Almén Nylander test, 56

Amines from amino acids, 110

Amino acid requirements, 200, 201

Amino acids, absorbed, 174

action of formaldehyde on, 99

action of nitrous acid on, 100

action with bases, 99

addition of acids, 99

as essential diet factors, 133

as source of urea, 183

conversion to amines, 110

fate of, 196

feeding with, 202

from proteins, 95

general properties, 98

glyeogen formers, 210

in blood, 195
A mino acids-Cont'd

isolation of optical isomers, 107

list and formulæ, 95-98

oxidation of, 100

per cent obtained from proteins, 95

Amino sugars, from lobster shells, 71

Ammonia in urine, 188

Amylase, pancreatic, 165

Anaerobic respiration, 57

Antiketogenesis, 205

Arabinose, 48, 68

Arginine, source of urea, 184

split by alkali, 102

Arsenic, 35

Ash, amount in body, 19

Assimilation limit, 207

Autocatalysis, 61

\section{B}

Basal metabolism, 219

Bases, 19

Bacterial action, intestinal, 170

Barfoed's test, 56

Barley sugar, 73

Benedict's test, 56

Beri-beri, 222

Bile, 162, 166-168

amount secreted, 166

cause of flow, 166

eomposition of, 167

effect on fat digestion, 165

effect on tryptic digestion, 164

in fat absorption, 175

rôle in digestion, 167

Bile pigments, 167

Bile salts, 168

Bilirubin, 167

Biuret, from urea, 182

Biuret test, 101

Birotation (see Mutarotation) 
Blood, 140-144 (see also Hemoglobin, ete.)

analysis results, 140

Blood, catalytic activity of, 118

coagulation of, 143

composition of, 140

corpuscles, composition of, 141

enzymes in. 142

gases, transference of, 141

general functions of, 140

guaiac test for, 119

hydrogen-ion concentration of, 143

laking of, 116

plasma, composition of, 141

platelets, 142

osmotic pressure of, 143

reaction of, 142

Bones, composition of, 138

Brain, eomposition of, 138 glucosides found in, 52

Breadstuffs, value as foods, 134

Breakfast foods, value as foods, 134

Bromine, 35 160

Buffers, 28

Butter, 87

Butter, value as food, 132

Buttermilk, 132

Butyric acid, 80

Calcium, 38, 193

blood elotting dependent on, 36

detection of, 39

distribution in body, 39

in elotting of milk, $36,39,115$, 160

phosphate in bone, 38

Calorimeter, 215

Cane sugar (See Saccharose)

Caprine, in brain, 138

Caramel formation, 53

Carbamino reaction of amino acids, 99

Carbohydrates, 19, 41-79

absorption of, 174

amount in body, 19

behavior with acids, 53

bchavior with alkalies, 52

ean be "'built down," 41

classification of, 48

combinations of, 52
Carbohydrates-Cont'd

composition of, 41

distribution of. 41

fats in bdy (See Metabolism of)

fermentation of. 58

general function. 41

individual groups. 67-79

interconversion of. 51

metabolism of. 53, 2ก3-2n?

metabolism $\mathrm{D}: \mathrm{N}$ ratio. 209

ocenrrence. 41

optical activity of, 42

origin of. 49

osazone formation, 57

oxidation of, 54

reduction of, 57

rôle in body, 203

rôle in plants and animals, 41

structure of, 41

synthesis in animals. 50

synthesis in laboratory, 50

svnthesis in rlants, 49

tests for 54-59

Carbon, 35, 36

Carbon monoxide hemoglobin. 121

Cartilage, composition of, 139

Caseinogen, altered by rennin. 159

Casein, digested by erensin, 165

general properties, 115

Cellulose, 48,77

food value. 78

value to body of, 134

Cerebron, in brain, 138

Cerebrosides, in brain, 138

Cheese, value as food, 133

Chewing, importance of, 146

Chlorides, 40, 190

Chlorine, 40

Chlorophyl, rôle in carbohydrate synthesis, 49

Cholesterol. 89, 90

in bile. 167

in brain, 138

Chromoproteins, 116

Chyme, 162

causes flow of bile, 166

Coagulated proteins, 92, 113

Cod liver oil, 87

Collagen, properties, 112

Collodial solutions, 28

Colloids, elassification and properties of, 29, 31 
Colloids-Cont'd

methods of precipitating, 32

Tyndall's phenomenon, 30

Conjugated glucuronates in urine, 180

Conjugated proteins, 92, 126-127

Connective tissue, composition of, 139

Cooking importance of, 131

Copper, 35

Cream, 132

Creatine, in brain. 138

in urine, 188-190

Creatinine, in urine, $188-190$

Cretinism, 227

Cyanhydrin synthesis of carbohydrates, 50

Cystin, bacterial destruction of, 171

\section{D}

Derived proteins, 92, 125-129

Dextrin, 48

general properties of, 77

in salivary digestion, 150

Dextrose (See Glucose)

Diabetes mellitus, 207

Diet, choice cf, 135

Digestion, general purpose of, 145

in intestine, 162-172

in mouth. 145-151

in stomach, 152-161

Disaceharides, 48, 72-75

\section{$\mathrm{E}$}

Eck's fistula, 184

Edestin, 112

Fggs, composition of, 133

Elastin, 113

Elements in body, 35

Emulsification, 182

Emulsions, importance of, 83

Energy exchange, 214

Energy requirements, 217

Enterokinase, 169

Enzymes, 59-66, 169

Enzymes vs. "'Ferments," 59

Erepsin, intestinal, 169 panereatic, 165

Ethereal sulphates in urine, 191

Excretion, general, 176

Extractives, 19, 20

Extractives in brain, 138

\section{F}

Fats, $80-87$

absorption of, 175

acetyl equivalent, 86

acrolein test for, 84

amounts in body, 19, 80

composition of, 80

detection, 84

distribution, 80

emulsification of, 82

formula of, 81

general properties, 82

identification of, 84

iodine number, 86

melting points of, 85

metabolism of, 211, 212

rancid, 84

Reichert-Meissl number, 85

saponification equivalent, 85

saponification of, 83

sources of, in body, 212

storage of, 211

volatile fatty acids in, 85

Fatty acids, 81

formed from carbohydrates, 58

Feces, composition and amount, 171

Fohling's test, chemistry of, 54

Fibrinogen, properties, 111

Field of physiological chemistry, 17

Fluorine, 40

Food accessories, 222

Food, definition of, 130

Foods, heat equivalent of, 216 specific dynamic action of, 217

Foodstuffs, 130-144

Formic acid, from carbohydrates, 52

Fructose, 70

conversion to other sugars, 51

distinction from glucose, 70

distribution, 70

Fruit sugar (See Fruetose)

Fruits, value as foods, 134

Furfurol, formed from earbohydrates, 53

G

Galactose, 70

formation from glucose, 70

Gall stones, 168

Gastric digestion, 152-161 
Gastric juice, 155

cause of flow, 153

Gastrin, 154

Gelatine, properties of, 113 in diet, 201

Gliadin, 112

Globulins, 92, 111

Glucosamine, 71

Glucose, 69, 71

conversion to other sugars, 69

formula of, 42

in blood, 174

in normal urine, 180

ultimate fate, 208, 209

Glucosides, 52, 79

Glucuronates, conjugated, formation of, 72

Glucuronic acid, properties, 71

Glutamic acid, feeding with, 204

Glutelins, 92, 112

Glycerine, in fats, 80

Glycocoll, built in body, 201

Glycogen, 48, 203-205

formed from various sugars, 51 general properties, 78

preparation of, 78

sources of, 209

stored in body, 204

Glycogen, yields glucose on hydrolysis, 51

Glycoproteins, 92, 114

Glycosuria, temporary, 208

Grape sugar (See Glucose)

Guaiac test for blood, 119

Guanine, $\mathbf{1 8 6}$

Guenzburg's reagent, 156

Gums, 48, 77

\section{$\mathrm{H}$}

Haines, test, 57

Half rotation, 47

Hard water for washing, 84

Hematin, 122

Hematoporphyrin, 123

Hemin test, 119

Hemochromogen, 122

Hemoglobin, 92, 116-125 (See Oxyhemoglobin, etc.)

Hemoglobin, absorption spectrum of, 120

as $\mathrm{O}_{2}$ carrier, 116-125

$\mathrm{CO}$ derivative of, 121

\section{Hemoglobin-Cont'd}

detection of, 118

fate of, in body, 123

molecular weight of, 117

source of bile pigments, 167

Hexoses, 69-72

H-ion concentration, 23

Hippurie acid, 187

Histones, 92, 113

Hopkins-Cole test, 102

Hormones, 154, 163, 199

Hydrochloric acid of gastric juice, 155,157

Hydrogen, 36

Hydrogen sulphide in sulphur test, 38

Hypoxanthine, 186

\section{I}

Imbilition, 34

Indicators, 26

Indol, 109, 171

Inorganic materials, $35-40$ metabolism of, 213, 214

Intestine, absorption in, 173-175 bacterial action in, 170

digestion in, 162.172

Inulin, 48, 77

Inverting enzymes, intestinal, 169

Iodine, 40

Iron, 38

Islands of Langerhans, 206

\section{K}

Keratin, properties, 112

Kidneys, and blood sugar, 205, 207

\section{L}

Lactase, pancreatic, 166

Lactose, 48, 74

Lanolin, 87

Large intestine, secretion of, 169 , 170

Lavoisier, 214

Lead, 35

Lecithoproteins, 92,125

Lecithin, 88

in brain, 138

Levulinic acid, from earbohydrates, 53, ј4 
Levulose (See Fructose)

Lipase of gastric juice, 160

Lithium, 35

Liver, and hemoglobin destruction, 39

Living material, characteristics of, 18

Lymph, 144

Lysine, need of, 201

\section{M}

Magnesium, 38, 193

Malt sugar (See Maltose)

Maltase, in blood, 174, 175

pancreatic, 166

Maltose, 48, 75

Manganese, 35

Mannose, 48, 51

Material bases, groups, 19

Meats as foodstuffs, 133

Mental activity and metabolism, 218

Metabolism, 194-227

general diseussion, 194

in sleep, 218

in starvation, 220-221

methods of study, 194

of carbohydrates, 203-209

of energy, 214-220

in disease, 219

of fats, 211

of inorganic materials, 213, 214

of proteins, 195-203

Metaproteins, 92, 126

in gastric digestion, 157

Methenoglobin, 121

Mett's tubes, 158

Milk, 120, 131

souring of, 132

Milk sugar, (See Lactose)

Millon test, 102

Mininum, law of, 35

Molisch reaction, 58

Monosaccharides, 48, 67-72

Mouth, absorption in, 173

Mucic acid test, 71

Mucilages, 48, 77

Mueins, 114, 149

IIucoids, 114

Murexid test, 185

Muscle, composition of, 135 contraction of, 135
Mutarotation, 47

Myosin, 112

Myosinogen, 112

\section{N}

Nerve, compounds in, 138

Neutral sulphur in urine, 191

Nitrogen, 35-37

Nitrogen balance, 197, 198

Normal solutions, 25

Nuclease, intestinal, 169 pancreatic, 166

Nucleic acid, 124 source of, 185

Nucleins, from nucleoproteins, 124

Nucleoproteins, 92, 123-125

Nuts, value as food, 134

\section{$\mathrm{O}$}

Oils, 82

Oleic acid, 81

Oleomargarine, 87

Optical activity, 46,47

Optical isomers, number possible, 48

Optimum temperature for enzymes, 60

Osazones, 57

Osmosis, 22

Osmotic pressure, 21

Oxalic acid, from carbohydrates, 52

Oximes, 51

Oxygen, 36

Oxyhemoglobin, spectrum, 120

crystallization of, 118

$\mathrm{P}$

Palmitic acid, 80

Pancreas and sugar metabolism, 205

Pancreatic juice, 162, 163

Paracasein, produced by rennin, 160

Pentoses, 67

Pepsin, 158-160

Peptids, 92, 128

behavior with enzymes, 108

precipitation of, 109

properties of, 108

synthesis of, 107-109 
Peptones, 92, 127

in blood, 196

in peptic digestion, 159

Phenol, gives Millon's test, 102

Phloridzin diabetes, 207

Phosphates, 38

in urine, 191

Phosphatides, 19, 87-90

Phospholipins, in brain, 138

Phosphoproteins, 92, 114, 115

Phosphorus, 36

Pituitary, and metabolism, 227

Plasmolysis, 22

Polariscope, 45

Polarized light, 43

Polysaccharides, 48, 75-79

Potassium, 38, 193

Prolamines, 92, 112

Proline, built in body, 201

in prolamines, 112

Protamines, 92, 113

Proteans, 92, 125

Proteins, 19, 91-129

absorption of, 174

amount in body, 19

amount required, 197-203

classification of, 92

color tests for, 101-103

elementary composition, 91

evidence for structure of, 108

form in which absorbed, 196

general reactions, 98-105

hydrolysis of, 94

hydrolysis not symmetrical, 109

importance, 91

individual groups, 110-129

metabolism of, 195-203

molecular weight of, 94

peptid linkage in, 106

precipitation reactions, 103-105

preparation of, 93 -

putrefaction of, 109

specific nature of, 203

storage of, 196, 197

structure of, 105-109

Proteoses, 92, 127

in blood, 195

in peptic digestion, 159

Protoplasm, properties of, 18

Ptomaines, 110, 170

Ptyalin, 149

Puncture diabetes, 205
Purine bases, from nucleic acid, 124

sources of uric acid. 185

synthesis in body, 187

Putrefaction, intestinal, 170

intestinal, reduced by bile, 167

of proteins, 109

Pylorus, control of, 161

Pyrimidene bases, from nucleic acid, 124

Pyruvic acid, 100, 197

\section{$\mathrm{R}$}

Rennin, 159-160, 165

pancreatic, 165

Respiratory quotient, 208

Rhamnose, 41

Ribose, 48

$\mathrm{S}$

Saccharose, $48,72-7 t$

Saliva, 146-151

Saponification of fats, 83

Secondary protein derivatives, 127 129

Secretin, 163

Sexual glands, and metabolism, 226

Silicon, 35

Silver, 35

Simple proteins, 92, 110-113

Skatol, 110, 170

Skin, 144

Soap, as cleansing agent, 84 as emulsifier of fats, 82

Soda lime, test for $\mathrm{N}, 38$

Sodium, 38, 193

Sodium chloride in diet, 39 phosphate, 38

Solutions, electrical properties of, 23

Specific dynamic action of foods, 217

Specific rotation, 43

Spleen, contains iron, 39

Starch, 48, 49, 76-77

digested by pancreatic juice, 165

digested by saliva, 150

Starvation, 220, 221

Steapsin, pancreatic, 165

Stearic acid, 80

Stokes reagent, 120 
St. Martin, Alexis, 153

Stomach, absorption in, 173 digestion in, 152-161 importance of, 152 why not digested, 161

Succus entericus, 162, 168

Sucrose, (See Saccharose)

Sugar in blood, 203

Sugar center in brain, 204

Sugar tolerance, 207

Sulphates, detection of, 38 in urine, 191

Sulphur, 36

in proteins, test fur, 103

Suprarenals, and blood sugar, 205

\section{$\mathrm{T}$}

Teeth, composition of, 138

Temperance of body, control of, 225

Temporary glyeosuria, $20 \mathrm{~s}$

Thymus, and metabolism, 227

Thyroid, and metabolism, 226 contains iodine, 40

Triolein, distribution, 87

Tripalmitin, distribution, 86

Tristearin, properties, 86

Trypsin, 164

Trypsinogen, 164

Tryptophane, body's need of, 201 destruction of, by bacteria, 170 in Adamkiewicz test, 102

Tyndall's phenomenon, 30

Tyrosine, bacterial destruction of, 170

in Millon's test, 102

need of, 201

\section{$\mathrm{U}$}

Unoxidized sulphur, test for, 38

Urea, 182-184

physiological action of, 184 sources of, 183

Urease, 183

Uric acid, 184-187

endogenous, 186

exogenous, 186

sources of, 185

variations in disease, 187

Uricase, missing in man, 186
Urine, 176-193

ammonia in, 188

earbonates in, 193

chlorides in, 190

color and transparency of, 178

consistency of, 179

creatine and creatinine in, $15 S$. 190

fermentation of, 180

hippuric acid in, 187

odor of, 179

optical activity of, 180

pathological constituents of, 193 (See Index of Laboratory Work)

phosphates in, 191

reaction of, 181

reducing power, 180

sediments, 178

specific gravity of, 179

sulphates in, 191

taste of, 179

total solids in, 179

toxic properties of, 181

urea in, 182-184

uric acid in, 184-187

volume of, 177

Urobilin, source, 167

Urochrome, 178

\section{V}

Value of. physiological chemistry, 18

Vegetables, as foodstuffs, 134

Vitamines, $221-225$

Vitellin, 116

Vividiffusion, 196

W

Water, 19, 40

absorbed in large intestine, 175

Waxes, 81

Work, and nitrogen excretion, 200

X

Xanthine, 186

Xanthoproteic test, 102

Xylose, 48

\section{Z}

Zein in diet, 201 


\section{INDEX OF LABORATORY WORK}

A

Absorption spectra, 281-28t

Acetone in urine, 353

Acrolein test, 266

Albumin crystals, preparation of, 274

Albuminoids, 277, 278

Albumins, test with, 274

Amino acids, 293

Atomic weights, table of, 359

\section{B}

Barfoed's test, 255

Benedict's test, 255 quality, 255 quantity, 225

Benzidene test, 284

Bile, 320-322

Biliary calculi, 321

Biuret test, 269

Blood, 279-289

benzidene test for, $28 t$

catalase in, 285

chemical tests for, 28t-286

serum, inorganic tests, 249

Blood analysis, 296

Blood sugar, 306-309

Bone, inorganic tests, 250

\section{C}

Calcium, test for, 251

Calibration, 236

Cane sugar, (See Saccharose)

Caramel test, 257

Carbohydrates, 254-263

Carbon, test for, 247

Carbonates, test for, 251

Caseinogen, 286

Catalase in blood, 285

Chlorides, test for, 250

Chlorides in blood plasma, 309

Cholesterin in gall stones, 322

Coagulated proteins, 291
Coagulation temperature of proteins, 275

CO hemoglobin, 282

Collagen, 278

Colloids, 245, 246

Congo red tests, 315

Conjugated proteins, 279-289

Creatinine, 302

Creatine plus ereatinine, 303

Cystin, 295

D

Derived proteins, 289-295

Dextrines, 262

Dextrose, 254-258

Digestion, in intestine, 319-322

in mouth, $310-313$

in stomach, 264-268, 314-318

Disaccharides, 260, 261

F.

Elastin, 278

Elements, 247-253

Emulsification, 265

F

Fats, 264-268

intestinal digestion of, 320

Fehling's test, qualitative, 254 quantitative, 350

Fermentation test, 257

Fibrin, 239

Folin's modified Nessler's Reagent, 296

Folin's test tube for estimating ammonia in urine, 333

\section{G}

Galactose, 259

Gall stones, 321-322

Gastric juice, composition of, 314 digestive action, 316-318

Gelatine, 278

General instructions, $53 \pm-235$

Gliadin, 277 
Globin, 286

Globulins, tests with, 275

Glutelins, 277

Glutenin, $2: 7$

Glycogen, preparation and tests, 263

Glycoproteins, 279

Gmelin test, 321

Guaiac test, 285

Guenzburg's test, 315

\section{$\mathrm{H}$}

Haines' test, $\Sigma 56$

Heller's test, 347

Hematin, 283

Hematoporphyrin, 283

Hemin crystals, 284

Hemoglobin, crystals of, 283, 284

Hemoglobins, 279:286

absorption, spectra of, 281-286

Histones, 278

Hopkins-Cole test, 270

Hydrochloric acid of gastric juice, 314-316

Hydrogen, test for, 247

\section{I}

Inorganic materials, 247-250

tests for, 250-253

Intestinal juice, 320

Inversion of saccharose, 260

Iodine test, 262

Iodoform test for acetone, 307

Iron, test for, 252

$$
\mathrm{J}
$$

Jaffe's test, 327

$$
\text { K }
$$

Keratin, 277

Kjeldahl nitrogen det' 1,241

\section{$\mathrm{L}$}

Lactose, tests with, 261

Lecithin, 267

Lecithoproteins, 289

Levulose, tests with, 258

Leucine, 294

\section{M}

Magnesium, test for, 251

Maltose, tests with, 260

Materials, 228-233
Metaproteins, 289-291

Methemoglobin, 283

Mett's tubes, 316

Milk, 286

coagulation of by rennin, 317

Millon's test, 270

Molisch test, 256

on proteins, 271

Motor power of stomach, 318

Mucic acid test, 259

Mucin, 279

Murexid test, 326

Muscle extract, preparation of, 248

Muscle residue, preparation of, 249

Myosin, 276

\section{$\mathrm{N}$}

Neutral oil, preparation of, 264

Nessler solution, 296

Nitrogen, test for, 248

Nonprotein nitrogen, determination of, 299

Normal solutions, 236-244

Nucleoproteins, 288, 289

$\mathrm{O}$

Optical activity, 257

Orein test, 259

Oxygen, 247

$$
\mathrm{P}
$$

Pancreatic digestion, 319-322

Pentoses, 259

Peptids, 293

Peptones, 292

Pettenkofer's test, 321

Phenylhydrazine test, .256

Phloroglucin test, 259

Phosphates, test for, 251

Phosphatides, 267, 268

Phosphoproteins, 286-28s

Pipettes and burettes, use of, 330

Polysaccharides, 261-263

Potassium, test for, 253

Precipitation tests, for proteins, 271

Prolamines, 276

Protamines, 278

Proteans, 289

Protein-free blood filtrates, preparation of, 297

Proteins, 269-295

biuret test, 269 


\section{Proteins-Cont'd}

color tests for, 269-271

gastric digestion of, 316

intestinal digestion of, 319

Millon's test for, 270

precipitation tests for, 271

xanthoproteic test for, 270

Proteoses, 291, 292

Ptyalin, action of, 311

R

Rothera 's test, 353

\section{S}

Saccharose, 260

Saliva, 310-313

Salivary digestion, 310-313

Saponification, 266

Sodium, test for, 251

Solubility, to test solubility of a substance, 254

Special reagents, $354-358$

Specific rotation, $\Sigma 58$

Standard acid and alkali, 237-245

Starches, 261, 262

Starch, digestion by ptyalin, 311313

Stomach, absorption from, 318 digestion in, 314-318

Succus entericus, 320

Sucrose, (See Saccharose)

Sulphates, test for, 251

Sulphur, test for, 248

\section{$\mathrm{T}$}

Toepfer's test, 315

Tyrosine, 294

\section{$\mathrm{U}$}

Uffelman's test, 315

Urine, 328-353

acetone bodies in, 353

acidity, estimation of (Folin), 331

albumin in, 346, 347

ammonia, qualitative test, 324

quantitative (clinical), 334

quantitative (Folin), 332

carbohydrates in, 349-353

carbonates, qualitative, 324

chlorides, qualitative, 323

quantitative (Volhard), 343

\section{Urine-Cont'd}

collection, 328, 329

colorimetric method for determination of urea in, 335

creatinine, quantitative (Folin), 340-342

creatinine, reactions of, 327

globulins in, 348

hippuric acid, 340

indican, 328,343

metaprotein, 349

nucleoprotein, 348

optical activity of, 353

oxalic acid, $327-328$

pathological, 346-353

phosphates, qualitative, 323 quantitative, 345

pigments, 328

preservation of, 328-329

proteins, quantitative, 347

tests for, $346-353$

proteoses and peptones, 349

purine bases, 340

reactions of, 327,328

qualitative tests, $323-328$

specific gravity, 329

sugar in, fermentation test, 352 quantitative (Benediet), 351 quantitative (Fehling), 350

sulphates, qualitative, 324 quantitative, 344

total nitrogen (Kjeldahl), 332

total solids, 331

urea, preparation of, 325

quantitative (urease), 334

uric acid, preparation of, 325 , 326

quantitative (Folin-Schaffer), 337-338

volume, 329

Urea, determination of, by urease, decomposition and distillation, 300

uric acid, 337,340

in blood, 304

Urochrome, 328

Uroerythrin, 328

\section{W}

Weyl's test, 327

$\mathrm{X}$

Xanthoproteic test, 270 



\section{UNIVERSITY OF CALIFORNIA}

MEDICAL CENTER LIBRARY

\section{THIS BOOK IS DUE ON THE IAST DATE}

\section{STAMPED BELOW}

Books not returned on time are subject to a fine of 50c per volume after the third day overdue, increasing to $\$ 1.00$ per volume after the sixth day. Books not in do mand may be renewed if application is made before expi-
ration of loan period. 


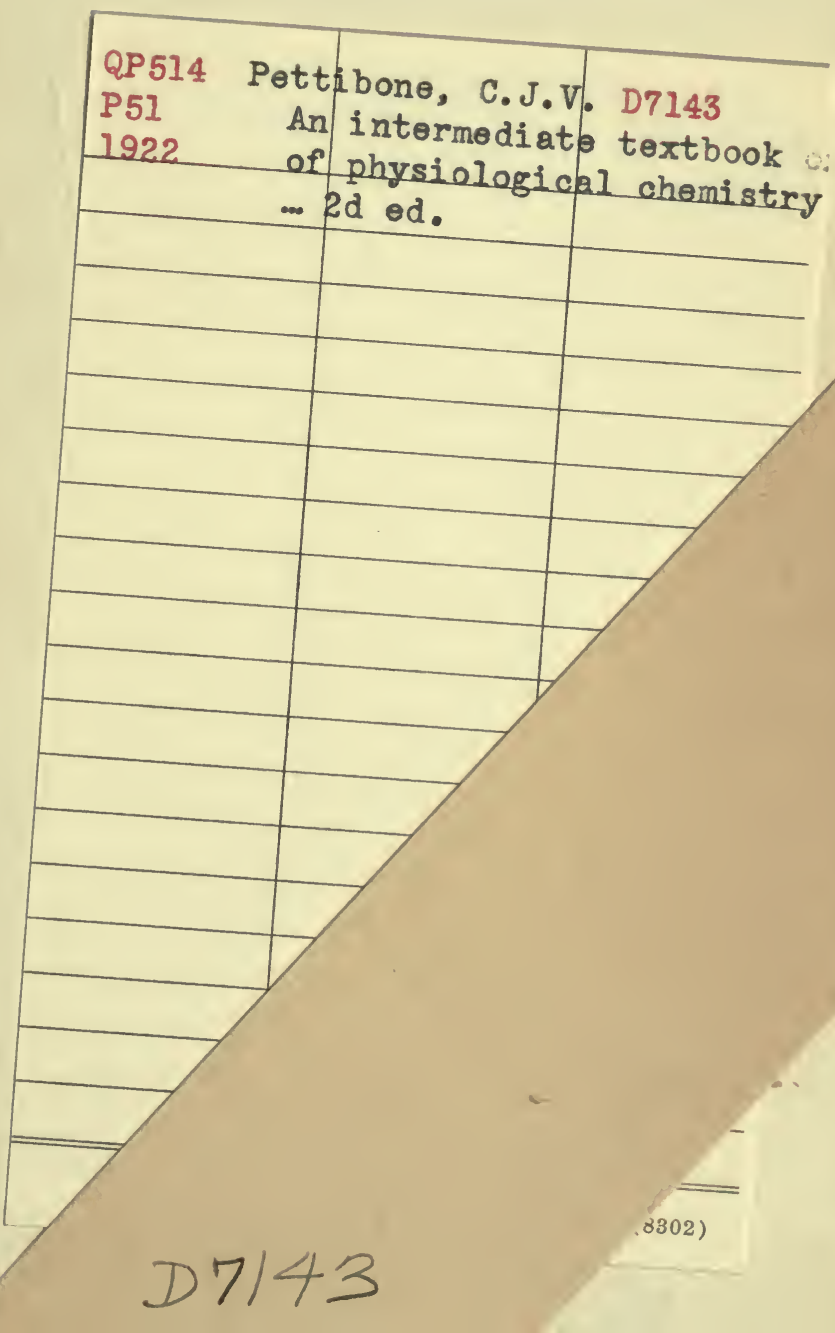


